Computation Rules
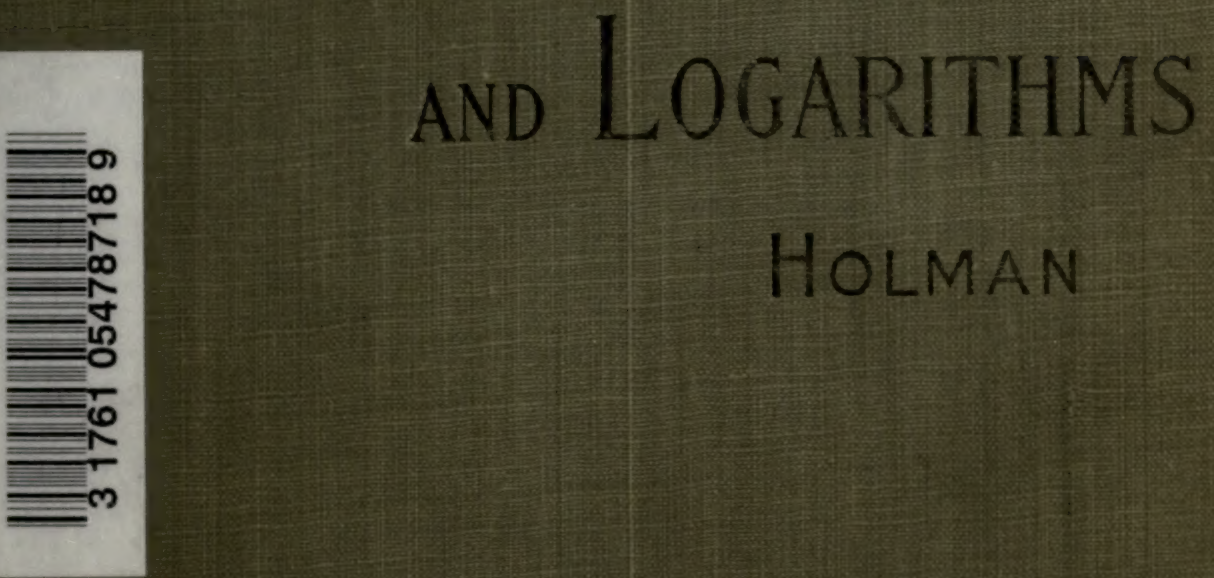

HOLMAN 



$$
7-5-5
$$


Digitized by the Internet Archive in 2007 with funding from Microsoft Corporation 
COMPUTATION RULES AND LOGARITHUS 
The $x$ : 4 . 


\section{COMPUTATION RULES}

AND

\section{LOGARITHMS}

WITH TABLES OF OTHER USEFUL FUNCTIONS

BY

SILAS W. HOLMAN

Professon or Prysics at the

Massachuberts Ixstixure oy TeohsoLog

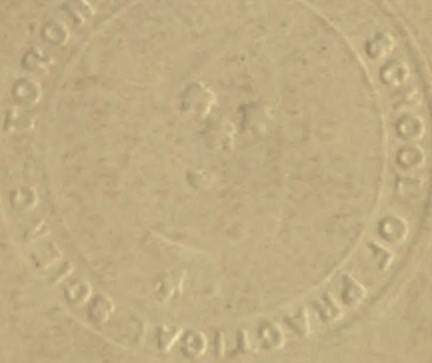

Nัrom Gork

THE MACMILLAN COMPANY

LONDON: MACMILLAN \& CO., LTD.

1896

AII rights reserved 


\section{Copyright, 1895,}

BY S. W. HOLMAN.

Set up and electrotyped December, 1895. Reprinted May, October, 1896.
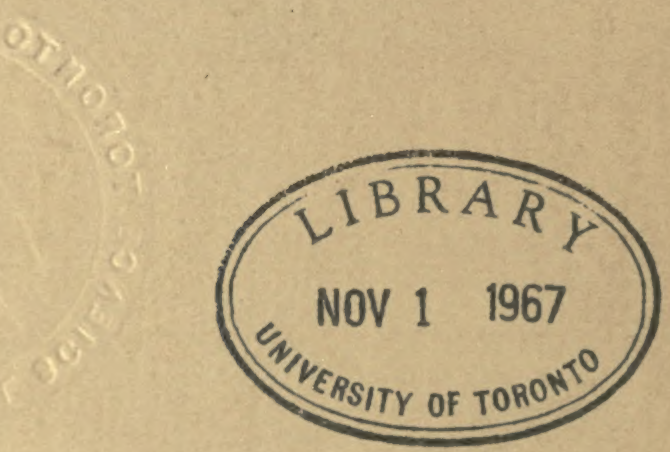

Norwoot 30ress

J. S. Cushing \& Co. - Berwick \& Smith Norwood Mass. U.S.A. 


\section{CONTENTS.}

PAGE

Preface * . . . . . . . . . . . . . . . . vii

Computation Rules . . . . . . . . . . . . . . . . xi

Proper Number of Places of Significant Figures . . . . . . xi

Fundamental Principles . . . . . . . . . . . . . xii

Rules in Detail . . . . . . . . . . . . . . . xiii

Rejection . . . . . . . . . . . . . . . . xiii

Multiplication and Division . . . . . . . . . . xiii

Logarithms . . . . . . . . . . . . . . xiii

Addition or Subtraction . . . ... . . . . . . xiv

Numerical Substitution in Formulæ . . . . . . . . xiv

Notation by Powers of Ten . . . . . . . . . . . $x v$

Statement of the Method . . . . . . . . . . . . xv

Symmetrical Grouping of Figures . . . . . . . . . . xvi

Examples I to II . . . . . . . . . . . . xvii

Logarithms. Nature of . . . . . . . . . . . . . xxiv

Tables, Description of . . . . . . . . . . . . . $\mathrm{xxv}$

To Find the Logarithm of a Number . . . . . . . . . xxvii

Decimal Point in Logarithmic Tables . . . . . . . . . . xxix

Anthogarithms: Number Corresponding. . . . . . . . xxx

Cologarithas . . . . . . . . . . . . . . . . xxxi

Habit in Reading off Numbers and Logarithms. . . . . xxxii

Powers and Roots by Logarithms . . . . . . . . . . xxxiv

Powers and Roots of Numbers greater than Unity . . . . . xxxiv

Powers of Decimal Fractions . . . . . . . . . . . . . xxxiv

Roots of Decimal Fractions . . . . . . . . . . . $\mathbf{x x x y}$

Squares and Square Roots . . . . . . . . . . . . xxxyi

REciprocals . . . . . . . . . . . . . . . . . $\mathrm{xxxviii}$

Natural Sines, Cosines, Tangents, and Cotangents . . xxxviii 
PAGE

Logarithms of Sines, Cosines, Tangents, and Cotangents . xxxix

Slide Wire Ratios . . . . . . . . . . . . . . . . . $\mathrm{xl}$

Defintmions and Explanations underlying the Computation

Rules . . . . . . . . . . . . . . . . . . xli

Significant Figures . . . . . . . . . . . . . . . . . xli

Places of Figures . . . . . . . . . . . . . . . . . xli

Places of Decimals . . . . . . . . . . . . . . . xlii

Accuracy; Reliability . . . . . . . . . . . . . . . xlii

Mean; Average. . . . . . . . . . . . . . . . . xlii

Deviation Measure . . . . . . . . . . . . . . . . . xlii

Rules for Significant Figures . . . . . . . . . . . . . . xliii

Rejection Error . . . . . . . . . . . . . . . . . . xliii

Law and Amount of Accumulated Rejection Error . . . . . . xliv

TABLES:

Logarithms, Four-Place . . . . . . . . . . . . . . 2

Anthogarithms, Four-Place $\quad . \quad$. . . . . . . . . . . 4

Cologarithms, Four-Place . . . . . . . . . . . . . . 6

Logartthas, Frve-Place . . . . . . . . . . . . . . . . 9

Square Roots and Squares, Four-Place . . . . . . . . 30

Reciprocals, Four-Place . . . . . . . . . . . . . 34

Sime Wire Ratios, Four-Place . . . . . . . . . . . 36

Natural Sines and Cosines, Four-Place . . . . . . . . . 38

Natural Tangents and Cotangents, Four-Place . . . . . 40

Logarithms of Sines and Cosines, Four-Place . . . . . . 42

Logarithms of Tangents and Cotangents, Four-Place . . . 44

Logarithms of Sines, Cosines, Tangents, and Cotangents,

Frve-Place . . . . . . . . . . . . . . 47

Constants . . . . . . . . . . . . . . . 70 


\section{PREFACE.}

IT would probably be within safe limits to assert that one-half of the time expended in computations is wasted through the use of an excessive number of places of figures, and through failure to employ logarithms. This waste might be almost wholly avoided by following a few simple computation rules and practising slightly with logarithm tables.

The loss from the use of superfluous figures will be appreciated when it is considered that in direct or logarithmic multiplication and division with four, five, and six places of figures the work is respectively in the ratio of $x: 2: 3$, or perhaps more nearly $2: 3: 4$. Thus contrary to the fallacious excuse so commonly given that it is just about as easy to use six- or seren-place tables as smaller ones, the work is doubled or trebled by the use of six places instead of four. Eren the employment of six- or seven-place tables, and dropping superfluous places when four or five are desired, causes much loss of time.

The proper employment of logarithms for work of four or more places effects a saving of one-quarter and upward of the time reçuired for direct multiplication or division, with a lessening of fatigue and a gain of accuracy.

The following pages contain simple rules to enable one to answer for himself the question, how many places of figures ought I to use in this computation? - also, an explanation of the use of the notation by powers of ten; certain instructions, more or less novel in form, as to the use of the logarithm and other tables: and a colleetion of useful tables. This eollection is designed to contain all the mathematioal tahles ordinarily reçuired, and nothing more, in practical work in all branches of the engineering professious, and by students of physies, chemistry, and engineering. for work of any grale not exceeding alout one-twentieth of one ber cent in accuracy. For 
many persons the present volume should, therefore, provide all the logarithmic and trigonometric tables needed for the entire range of their practice. For work of greater precision than the above limit, the more bulky Vega, or some similar reliable seven-place table would be required. It is exceedingly rare that more than six or seven places are necessary, while for most work five are sufficient, although a striking chapter of absurd illustrations might be gleaned from various text-books and tables where ten- and even twenty-place logarithms are given, often for quantities uncertain in their fourth or fifth place. Persons doing much work with squares, cubes, square roots, cube roots, or reciprocals of more than four places would naturally make use of the Barlow Tables.

The rules for significant figures (pages xi to $\mathrm{xv}$ ) are intended to be terse, direct, and simple, so that they may be easily acquired and retained. The strong type emphasizes the leading portions. The ordinary and finer types give details and explanations. For the sake of affording still greater prominence to the main working portions, some explanatory matter which will be unnecessary to many persons has been transferred from its more logical position of precedence to the latter part of the text. These rules in various forms have been in successful use by large classes of students, in connection with the author's "Physical Laboratory Notes" (printed, but not published, by the Massachusetts Institute of Technology), and his "Precision of Measurements." The recognition of the need of such rules amongst engineers and others whose practical work demands rapid and reliable computations was the cause of their general introduction into this laboratory instruction. It is therefore hoped that they may render effective service to others besides the students for whom they have been more directly designed.

In the arrangement of the tables, the effort has been exerted to make them correct, legible, systematic, and convenient in use. A new set of tables is, of course, liable to contain mistakes; notices of errata will therefore be thankfully received.

The special indexing of the corners of pages, the use of heavy type at points to be made conspicuous, the employment of spaces rather than rules for the partition of lines and columns, and the style of type and kind of paper used, are believed to conduce to legibility. As to system of arrangement, there are few novelties other than the insertion of the logs, cologs, and reciprocals of 1.000 to 1.100 at the top of the respective four-place tables, and the division 
of most of the four-place tables so that the second page begins with 5.0 instead of the customary 4.5. The frequent oceurrence of cor rection and reduction factors, ranging from $\mathbf{x} .0$ to $\mathrm{x} . \mathrm{I}$, renders this by far the most frequently used part of the table; while at the same time, on account of the large tabular differences, interpolation is here the most laborious. The insertion of logs, cologs, and reciprocals from r.o to r.1 with increments of 0.001 and 0.000 r, respectively, in the four- and five-place tables, obviates this interpolation. In tables of antilogs and square roots the addition would be of little service. In the tables of logarithms and of square roots, heavier type has been used at apparently scattered points throughout the body of the tables. These points, in the five-place logarithm tables, for instance, are where the first two figures in the mantissa change by one unit in the second place, e.g. oo, or, o2, etc. The obvious service of this is to aid the eye in finding any desired mantissa in working the table backward to obtain the antilog or number corresponding. The object is, of course, the same in the other tables.

As to the wisdom of departing from the usual custom of onitting decimal points entirely from logarithm tables, the author helieves that the retention of the point promotes clearness of comprehension of the tables by beginners, and lessens mental effort in more experienced computers, especially when associated with the notation by powers of 10 , as in the explanations here given. It seems unfortunate that this simple notation, so useful in computation and so great an aid in the explanation of numerical relations, is not universally incorporated into arithmetical instruction.

The rules for the employment of logarithms and of the tables have not been prepared especially to meet the need of those entirely unfamiliar with the prineiples of logarithms, although they would probably be intelligible to any mature heginner. It is thought, however, that the explanations and instructions given may prove an aid even to those who are alrealy somewhat familiar with the subject.

Roorrs Laboratory or PuYsics,

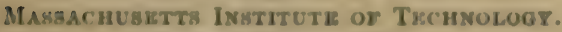

Boston, August, 1806. 



\section{COMPUTATION RULES.}

\section{PROPER NUMBER OF PLACES OF SIGNIFICANT FIGURES.}

THe following three pages contain the rules and their underlying principles in a condensed form for ready reference. For readers to whom some of the terms employed are unfamiliar, or who desire fuller proofs and explanations, some additional pages of "Definitions and Explanations" have been appended.

These rules should enable a computer to decide at the outset of his work, or at the successive stages of it, what number of places of significant figures he should retain in order to avoid waste of labor on the one hand or sacrifice of accuracy on the other. They provide for a sufficient number of places to assure that (harring mistakes) the aurumulated error arising from the rejection of further places shall be always smaller, usually much smaller, than the supposed uncertainty of the data or result, in computations involving not more than about 20 rejections. The retention of more places is worse than useless. It adds nothing to the accuracy of the result, although increasing materially the labor of computing, and the liar bility to mistake. The aggregate value of the time thus wasterl, -obvious enough to any one who has had oecasion to perform extended computations, - may be appreciated from the fact that the use of tive places where four would suffice, nearly douhles the lahor: using six places insteal of four, nearly trehles it; thus wasting roo and 200 per cent respectively of the necessary amount of work, and probably a greater proportion of time. Moreover, incongruities in the use of places of figures arouse skepticism as to the competence of the worker in other directions. 


\section{FUNDAMENTAL PRINCIPLES.}

Retain everywhere enough places to correspond to two unreliable places in the final result; the direct object of this is to keep the first place of unreliable figures in the final result substantially free from the accumulated rejection errors.

Exieptroxs. - A final result is seldom stated to more than one uncertain place unless the uncertainty of that place is small (say plus or minus four or less).

\section{Example: 1, page xvii.}

Single direct measurements generally yield numbers extending to only one uncertain place. This should not, however, be taken as a reason for relaxing the application of the above rule to subsequent steps of the computation, especially in deducing the mean or average of several single observations.

Final zeros occurring in decimal fractions should be retained when any other digit in the same place would be retained. This is of course essential to show that this place is known.

The foregoing principles consistently carried out constitute entirely sufficient rules. But more detailed instructions are usually required at the outset. These are readily understood in view of the two following propositions, which one can easily verify by algebra or by numerical examples.

Proposition I. - In multiplication or division, the percentage accuracy of the product or quotient cannot exceed that of the factor whose percentage accuracy is least.

Proposition II. - In addition or subtraction, the result cannot be accurate beyond the first decimal place which is inaccurate in any component.

A more general form of statement from which these follow is: If several numbers are multiplied or divided, a given percentage error in any one of them will produce the same percentage error in the result. If several numbers are added or subtracted, a given error or change in the digit in any decimal or other place will produce an equal error or change in the digit in the same decimal place in the result. 


\section{RULES IN DETAIL.}

Resectios. - In casting off places of figures, increase by I the last figure retained when the first left-hand rejected figure is 5 or greater; otherwise leave it unchanged.

\section{Example. - If the last two figures are rejected}

$\begin{array}{llll} & 756827.9 & \text { becomes } & 756830 . \\ \text { and } & 0.00263439 & \text { becomes } & 0.002634 .\end{array}$

A MEAN OR AVERAGE should always be carried to two unreliable figures.

A mean is more reliable than the single observation from which it is computed (in proportion to $\sqrt{n}$, the square root of the number of observations). Thus, as the data frequently extend to only one unreliable figure, the mean will often have to be carried two places further than the single observation.

Multiplication or Division. - Ascertain from the object of the work the percentage accuracy desired in the final result; or, by inspection of the data, find the percentage accuracy of that factor for which this is least, i.e. for which the deviation-measure or the estimated error, expressed as a per cent, is largest. See Example $\mathbf{x}$, page xvii.

In direct multiplication or division retain in every factor, product, and quotient throughout the entire process, and in final results, for an accuracy of about

One per cent, or worse, four (4) places of significant figures;

One-tenth per cent, or worse, five (5) places of significant figures; and so on.

In the ordinary and the shortened processes of "long multiplication," it is best to carry out the partial products one place beyond that yielding the last place required in the result under the above rule.

Examples: 2-5, page xvii.

Logarituss. - If the multiplication or division is performed by means of logarithms, the mantissa should contain as many places as are required by the foregoing rules for the direct process; 1.e. for about

One per cent, or worse, use four (4) place tables;

One-tenth per cent, or worse, use five (5) place tables.

Examples: 2-5, page xvi. 
Andition or Simtraction. - Ascertain from the stated object of the work the percentage accuracy desired. If this is about

One per cent, or worse, carry the result to four (4) places of significant figures;

One-tenth per cent, or worse, carry the result to five (5) places of significant figures; and so on, and carry each component quantity to that place of decimals which would correspond to this required place in the result, that is, stand in the same column with that place.

Examples: 6-8, page xix.

When the DEsTred ACCURACY IS Not STATED, inspect the data to find the component whose first uncertain place is furthest to the left, i.e. whose deviation measure (page xlii), in units, not percentage or fractional, is greatest. Retain this component to two uncertain places, and all other components to the place which would stand in the same column with this second place.

Examples: 6-8, page xix.

N. B. - If the number of components approaches 20 , care may well be taken in refined work that an unusually large rejection error does not enter through a special combination of rejected figures. The rules are, however, sufficient for the worst possible case.

The computer should notice that the percentage precision of a result which is the difference of two or more quantities will usually be smaller, and may be much smaller, than that of any of the component quantities.

Numerical Substitution in Formulæ. - A large number of formulæ may be represented by the type

$$
x=\frac{a \cdot b \pm c \cdot d \pm \cdots}{p \cdot q \pm r \cdot s \pm \cdots}
$$

where $a, b, c, d$, etc., represent numbers to be multiplied, divided, added, or subtracted, etc., as indicated. Any one or more of the factors and terms may be wanting; or, there may be several in place of two; and so on.

Obviously, in order that the result $x$ shall be accurate to a specified per cent, both numerator and denominator must be at least of that accuracy, and each should therefore be carried out to the number of places of significant figures needed in $x$. Then as the numerator consists of two or more terms $a b$ and $b c$ added or subtracted, 
inspection under the foregoing rules for addition or subtraction will show to what decimal place each of these terms must be carried. Further, $a$ and $b$ must each be carried to the number of significant figures thus required in the product $a b$, and so on.

In complieated formulie this process of inspection is sometimes slightly troublesome, but is essential unless the necessary precision of the components has been otherwise studied; as, for example, by the simple applieations of the differential calculus as in the author's "Precision of Measurements."

Examples: 9-II, page $\mathrm{xx}$. Practical examples of substitution in moderately simple formulæ.

\section{NOTATION BY POWERS OF TEN.}

Statement of the Method. - Regard the decimal point as merely an affix whose sole purpose is to indicate which is the units' place of figures. Fix the attention firmly upon the units' place as the centre of symmetry of our customary system of notation. The too universal reference to the decimal point, rather than to the units' place, in arithmetical rules and explanations, has resulted in masking this symmetry and in thus depriving the student of its important aid. In our common decimal system of notation, a digit in the units' place represents so many times unity, i.e. so many times $10^{\circ}(=1)$, or so many units. In the first place to the left of the units' place the digit represents so many times $10^{+1}$, i.e. so many tens, and in the first place to the right, so many times $10^{-1}$, i.e. so many tenths; in the second place to the left so many times $\mathrm{ro}^{+2}$, i.e. hundreds, and to the right so many times 10 2, i.e. hundredths; in the sixth places, so many times $\mathrm{ro}^{+6}$ and $\mathrm{ro}^{-6}$, i.e. millioms and millionths, respectively: and so on. The fundamental symmetry of the whole system about the units' pluce is thus obvious, and should not be lost sight of.

In counting up places, whether to right or left, always begin with the units' place as zero.

It is clear, then, that we may write numbers in this way:

for 90

for 6000

for 345

for 0.00005

for 0.004689

for 850.72 write $9^{\circ} 10^{1}$;

write $6.000 \cdot 10^{3}$ or $6.10^{3}$ as the case may require;

write $3.45^{\circ} 10^{2}$;

write $5 \cdot 10^{-8}$;

write $4.689^{\circ} \mathrm{ro}^{-0}$;

write $8.5072 .10^{2}$; and so on. That is, 
Separate the number into two factors, the first being the original number with the decimal point changed in position so as to follow the first figure; the other being $10^{ \pm n}$, where the sign is plus for a whole number and minus for a fraction, and where $n$ is the number of places the decimal point has been moved.

To transform a number expressed in this way back into the ordinary form, move the decimal point $n$ places, making the number a whole (or a larger) number if $n$ is plus, and a fraction if $n$ is minus.

Associate firmly in the mind the plus sign with whole numbers, the minus sign with fractions; thus avoiding confusion as to the sign of $n$.

In much work, the factoring need not be written out, but may merely be mental.

This notation reduces the error and work of locating the decimal point in multiplication or division, especially in expressions containing several terms in the numerator and denominator. It is very helpful in connection with the characteristic of logarithms, and the location of the decimal point in evolution, involution, and finding reciprocals. It saves space and promotes clearness in expressing large numbers or small fractions, and it is the best aid in following the decimal point while using the slide rule. It also enables one to dispense with characteristics in certain parts of computations (see Examples, page xxi).

An abbreviated notation helpful in one's own work, but perhaps not to be urged for general adoption, consists in dropping the $\cdot 10$, thus,

$$
\begin{array}{llll}
\text { instead of } & 4 \cdot 507 \cdot 10^{2} & \text { write merely } & 4 \cdot 507^{2} \\
\text { instead of } & 5 \cdot 3704^{\cdot 10^{-8}} & \text { write merely } & 5 \cdot 3704^{-8}
\end{array}
$$

The adoption of the bracket or parenthesis, e.g. $(4.507)^{2}$, for either notation in cases of possible doubt removes all risk of mistaking these indices for ordinary exponents of powers.

Examples 9, IO, II give incidentally illustrations of the use of the notation by powers of $\mathrm{xo}$

Symmetrical Grouping of Figures. - For writing numbers, adopt the following system of groups and spaces:-

$$
\begin{aligned}
& \text { Write } 143258.64796 \\
& \text { instead of } 143,258.647,96 \text {, the usual method. }
\end{aligned}
$$

A still clearer method would be to write

\section{I4325864796}

denoting the units' place by the heavy figure, but this is impracticable. The proposed system is symmetrical about the units' place, the customary system. is not. It groups together the units, tens, and hundreds of thousandths, of millionths, ete., as well as of thou- 
sands, millions, etc. It is clearer and less liable to error by the substitution of spraces for the commas to mark off the groups Exception is usually to be made in the case of a decimal fraction containing only three or four places. Thus write 0.4612 rather than 0.4612 , and 6.382 rather than $6.3^{8} 2$.

\section{EXAMPLES.}

Example 1. - Suppose that a final result was stated as 298549. \pm 0 . Io per cent; this would mean that its uncertainty or deviation-measure or estimated measure of accuracy (see page xlii) was \pm 0.10 per cent. To how many places should it be retained? 0.1 per cent of the number is $0.001 \times 300000=300$. Therefore the last three places are uncertain, but as the uncertainty in the first left-hand one is small (3), two uncertain places should be retained. The result, therefore, should be written 298550 . \pm 0.10 per cent.

Suppose a result given as $47.582435 \pm 0.0062$. This would be an incorrect. use of figures. The \pm 0.0062 shows that the result is uncertain in the third and fourth, and therefore in all subsequent deeimal places.* The fifth and sixth places of significant figures are thus unreliable, so that the seventh and eighth places are entirely valueless, and should, therefore, be rejected. We should be at liberty to use our judgment as to whether the result should then be written

$$
47.5824 \pm 0.0062 \text { or } 47.582 \pm 0.006
$$

since the uncertainty in the fifth place is large. The second is more common practice. In this example the uncertainty is $\pm 0.0062 / 47 .= \pm 0.00$ or 3 , or \pm 0.013 per cent. It might, therefore, have been expressed as \pm 13 parts in 100,000 , or \pm 0.013 per cent instead of as \pm 0.0062 units. It is always expressed in the saue unit as the quantity itself, e.g. ft., lbs., etc., except when directly stated to be a percentage.

Example 2. - Desired with an aceuracy of 2 per cent, the volume of a right circular cylinder whose radius $r$ is 6.0428 inches, and length $l 12.653$ inches. Volume $V=\pi r^{2}$. By the rule for multiplieation, since the result is desired to worse than one per cent, the data and all steps should be carried to four places of significant figures. Hence, we should have

$$
V=3.142 \times 6.043^{2} \times 12.65=1451 \text {. }
$$

By ordinary multiplication:

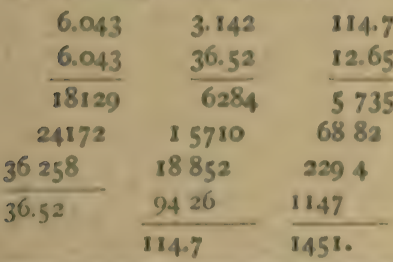

By shortened multiplication:

\begin{tabular}{rrrr}
6.043 & 3.142 & 114.7 \\
6.043 & & 36.53 & 1265 \\
\hline 36.258 & & 94.26 & 1147 \\
240 & 1884 & 2294 \\
18 & 155 & 690 \\
$\frac{16.52}{36.5}$ & & 6 & 55 \\
\cline { 3 - 4 } & 114.7 & 1451.
\end{tabular}

- This quantity so.cons2, or whatever may he its value, is the "average deviation" (If the "deviation-lurasure" of the result: that is, the average amount by which seseral results similarly olifained would differ from their mean. A fulier explanation is given at page xlii. The "probable error." which is nesarly blention with the arerage deviation, is commonly used in its stead. Vither sustices. 
Observe that the partial products beyond the place standing over the fifth place of the result in each multiplication are useless. Hence the obvious saving of labor in the shortened process, which is also more compact. The process is easily understood by inspection of the example. Multiply first by the first lefthand figure of the multiplier. If the resulting partial product has one more place than is desired in the result, then drop the last figure of the inultiplicand when multiplying by the second figure of the multiplier; drop the last two, when multiplying by the third figure; and so on. If, however, the first partial product has not the desired number, the dropping of figures must be deferred till the third figure of the multiplier is used.

Example 3. - Desired the volume $V=\pi r^{2} l$ of a right circular cylinder whose dimensions are

$$
r=6.0428 \pm \frac{1}{4} \text { per cent, } l=12.653 \pm \frac{1}{10} \text { per cent. }
$$

The result cannot be more accurate than the least precise factor, which is obviously $r$. Under the rule, + per cent computations call for five places of significant figures. Hence we should have to find by multiplication or by fiveplace logarithms,

$$
V=3.1416 \times(6.0428)^{2} \times 12.653 .
$$

Note in this connection that the error in $V$ is proportional to twice the percentage error in $r$, since $r$ enters in the second power, and that in general where a number is raised to any power $n$ the percentage error in the result is increased to $n$ times its value in the data. These rules, however, provide sufficiently for such cases. See "Definitions and Explanations."

Example 4. - Desired the volume $V=\pi r^{2} l$ of a right circular cylinder whose dimensions are given as

$$
r=6.043 \pm 0.017 \text { inches, } l=12.653 \pm 0.038 \text { inches. }
$$

Under the general principle of retaining places to correspond to two uncertain figures in the result in the data, $r$ should have four places and $l$ five places, judging from their stated precision. But the weaker quantity fixes the number of places in the result, so that we should use but four places:

$$
V=3.142 \times(6.043)^{2} \times 12.65 .
$$

Example 5. - Desired the ratio of the radius to the length in each of the Examples 2, 3, and 4.

The number of places of figures to be used would be respectively four, five, and four, just as in the above solutions. A factor in the denominator follows precisely the same rule as to places of significant figures as the one in the

\begin{tabular}{|c|c|}
\hline \multirow{2}{*}{$\begin{array}{r}6.043 \\
5060\end{array}$} & 12.65 \\
\hline & 0.4777 \\
\hline 9830 & \\
\hline 8855 & \\
\hline 975 & \\
\hline 889 & \\
\hline 86 & \\
\hline 91 & \\
\hline
\end{tabular}
numerator. To contrast the ordinary and shortened solutions, the following are given :

$$
\begin{aligned}
& \text { 12.65) } 6.043(0.4777 \\
& \frac{5060}{9830} \\
& \frac{8855}{9750} \\
& \frac{8855}{8950} \\
& 8855
\end{aligned}
$$


Example 6. - Desired the value to 0.6 per cent of

$$
47 \cdot 3489+174 \cdot 32825-5.62147 .
$$

For o. I per cent or worse (page xiv), we must retain places in the compo. nents to correspond to five places in the result. By inspection we see that the result will be slightly more than 200 . Hence its fifth place will be the second decimal place, and we need retain no place beyond that in the components. Thus,

47. 35

174.33

221.68

$-5.62$

$\overline{216.06}[ \pm$ an unknown amount as precision-measure].

Example 7. - Desired the algebraic sum of

$47^{\prime} \cdot 3489 \pm 0.004^{2}, \quad 174^{\prime} \cdot 32 \$ 25 \pm 0.000819, \quad$ and $-5^{\prime} \cdot 62147 \pm 0.00008$.

By inspection the weaker conponent, that is, the one whose first uncertain place is furthest to the left, is the first number. Retaining this to two uncertain figures would carry it to the fourth decimal place. It will then be useless to retain the other components beyond that place, and we shall have

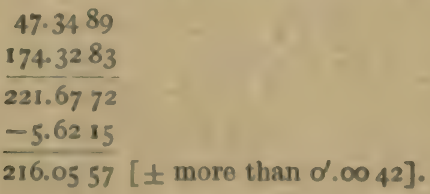

Example 8. - Desired the algebraic sum of

$47^{\prime} \cdot 3489 \pm 0.05$ per cent, $174^{\prime} \cdot 32 \delta_{25} \pm 0.02$ per cent, $-5^{\prime} .62147 \pm 0.1$ per cent.

0.05 per cent of 47 . is $0.024 ; 0.02$ per cent of 174 . is $0.035 ; 0.1$ per cent of 5.6 is 0.0056 . Hence the weakest eomponent is now the second, and this, and conserumenty the others, should be retained to three decimal places. Thus, we have

$$
\begin{aligned}
& \begin{array}{r}
47.349 \\
174.328
\end{array} \\
& 221.677 \\
& -5.621 \\
& 216.056\left[ \pm \text { more than } d^{\prime} .035\right] \text {. }
\end{aligned}
$$

Example 9. - The horse-power, IIT, which could safely be transmitted by a wrought-iron shaft of diameter $d$ inches, ruming at a speed $X$ rotations per minute, the safe shearing loal of wrunght iron being represented by $f$, is given by the expreasion

$$
U P=\frac{2 \pi^{2} d^{n} f X}{16 \cdot 12^{\circ} 33000}
$$


[Deduced from Lanza's "Applied Mechanics," page 336. The several constants $2,16,12$, and 33000 would of course be combined into a single constant in a working formula, but they are here left separate for purposes of better illustration.]

To how many places of significant figures should the quantities, result, and varinus steps of the computation be carried out to assure against a computation error in the result, sensible as compared to one per cent?

Solution. - In this and all similar problems, where the expression consists merely of a number of factors in the numerator and denominator (either or both), without additions or subtractions, the solution of the significant figure problem can be made without any knowledge of the magnitude of the component quantities, such as $d, f, N$, etc. In this example, as the result is desired to one per cent, aceording to the rules it should be carried to four places of significant figures. Hence, according to the rule, page xiii, or to Proposition II, page xii, each factor of the whole expression should be carried to four places. In this expression every quantity is a factor, either in the first or a higher power, viz. 2, $\pi^{2}, l^{3}, f, N, 16,12$, and 33000. Each, therefore, should be carried to four figures. Hence, also, if direct multiplication be employed in the solution, each product and quotient must be carried to four places. If logarithms are used (they should be) four-place tables should be chosen. When a quantity enters as a factor of the $n$th power this is equivalent to its entering $n$ times as a simple factor or as $n$ separate factors, all with the same percentage error of the same sign. See also note under example 3 .

The constants 2, 16, 12, and 33000 do not require to be carried to more places than they are here given because they are complete as they stand, that is, all further figures are known to be zero as a matter of definition or mathematical fact. If either of them had been an experimental constant, that is, determined by measurement, it should have been carried out to four places even if the last figure or two were zero. For instance, if experimental, the 16 should have been written $16.0,16.00,16.000$, and so on according to the number of places to which it was known (see rule, page xii). Failure on the part of those who write such formulic to adhere to this convention, or to indicate in some clear way the degree of accuracy possessed by such constants, is a serious source of amnoyance and trouble to those who use them.

As elsewhere it must not be inferred if certain of the quantities, e.g. $d$ or $f$, in this expression cannot be carried out to this desired number of figures, that consequently the result will not have the accuracy desired in the given case. The outcome of such a condition would merely be that these factors would be liable to introduce more than a safe computation error. For instance, if $f$ were given as 10100 lbs. per square inch, we should have no certainty that it was carried far enough. The presumption would be that it was correct to but three places, and therefore not exact enough. If, however, from a knowledge of the subject we were aware that the best known value was ror 1o, we should know that the error from using Ioloo was only $\mathbf{I}$ in 1000 or $0 . \mathbf{I}$ per cent, and hence admissible. (On the other hand, if we know that the best value was roo50, we should know that the computation error in the result from using 10100 was 0.5 per cent, and hence by no means safe in the above problem.

More complete methods for ascertaining the exact accuracy needed in each component measured quantity in such formula, are given in the author's "Precision of Measurements." It is to be remembered that we are now dealing 
merely with rules for computation errors, and these are not suited to the other problem. They are intended to secure a safe number of pluces fur the vourst cuse, and would, therefore, impose unduly severe requirements as to the accuracy necessary in the metsurement of the components in most cases.

Vumerical Sulstitution. - The numerical expression to be solved if written out would be

$\frac{2 \cdot 3 \cdot 142^{2} 1.364^{2} \cdot 10000 \cdot 300}{16 \cdot 12 \cdot 33000}$ in the ordinary notation;

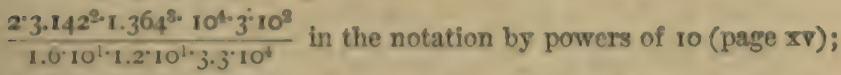

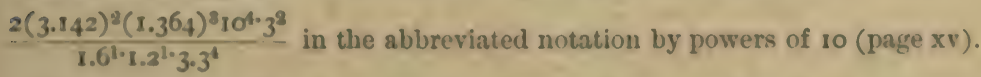

The first would be worked out in the usual manner by direct multiplication or by logarithms, as shown below.

The second would be worked as follows:

Multiply together the terms other than Ion $^{\text {m }}$ of the numerator, i.e.

Mfultiply together the terms other than $10^{n}$ of the denominator, i.e. $2 \times 3.142^{2} \times 1.364^{8} \times 3=150.3$

$1.6 \times 1.2 \times 3.3=6.336$

Divide numerator by denominator $=23.7^{2}$

Add together all indices of powers of 10 in numerator, also in denominator, and subtract the latter from the former. (Or, better, add (algebraically) all the indices, reversing the sign of those in the denominator, thus : $4+2-1-1-4=0$.

The result is therefore $23.72^{\prime} \cdot 10^{\circ}$

Sote distinctly that all this writing out of the fraction and of the several steps is merely for the purpose of this explanation. In an actual solution such of these uperations as are essential to the work would be conducted mentally, the artual multiplieation and division alone being written out.

If the solution were made by logarithms, it might assume either of the two following forms. The first is the usual one, the second shows how the use of the powers of to enables us, if we choose, $t_{1}$ dispense with writing characteristics in very many places, - a saving of just so much labor. The factor $10^{6}$ in the second result is, of course, ohtidined by summing mentally the indices of the factors 10, thuse in the demminatur being taken with reversed sign, as in the preceding paragraph. These indices would not be written out, but taken by direct inspertion of the data as originally written.

\begin{tabular}{|c|c|c|c|c|c|}
\hline Destimuinator. & $\begin{array}{l}\text { Vasaul } \\
\text { Metheul. }\end{array}$ & $\begin{array}{l}\text { Jropyilug } \\
\text { Clusactertaties. }\end{array}$ & Sumerator. & $\begin{array}{l}\text { Tsual } \\
\text { Metheut. }\end{array}$ & $\begin{array}{l}\text { Dropping } \\
\text { Charaeteristie. }\end{array}$ \\
\hline $\log 16$ & $=1.2041$ & .2048 & $\log 2$ & $=0.3010$ & .3010 \\
\hline $\operatorname{lng} 12$. & $=1.0792$ & .0792 & $2 \times \log 3.142$ & $=0.9944$ & .9944 \\
\hline log 33 noo. & $=4.5185$ & .5185 & $3 \times \log 1.364$ & $=0.4044$ & .4044 \\
\hline \multirow{6}{*}{ log denon. } & $=6.5018$ & $.801 \overline{8}$ & $\operatorname{lig} 10 \mathrm{con}$. & $=4$. & \\
\hline & & & $\log 300$ & $=2.4771$ & -4771 \\
\hline & & & & 8.1769 & 2.1769 \\
\hline & & & & 6.8018 & .8018 \\
\hline & & & & 1.3751 & 1.3751 \\
\hline & & & Reanit, & $23.7^{2}$ & $23.72 .10^{\circ}$ \\
\hline
\end{tabular}


Example 10. - The crushing load of a hollow, cast-iron pillar of circular section, with concentric surfaces of diameters $D$ and $d$ as given by Hodgkinson (Lanza, "Applied Mechanics," page 332) is

$$
c=109801 \frac{\pi\left(D^{2}-d^{2}\right)}{4} \text {. }
$$

Desired to ten per cent the load which a pillar of external and internal diameters 4.032 inches and 2.16 inches, respectively, would carry. How many places of figures should be used in the computation?

Ten per cent results call for three figures in all factors (page xiii). The factors in this expression are $10980 \mathrm{r}, \pi,\left(D^{2}-d^{2}\right)$, and 4 , each of which should therefore be carried to three figures. The first two should therefore be 110000 and 3.14. The 4 is a complete number as it stands. $D^{2}-d^{2}=4.0^{2}-2.2^{2}$ roughly $=16.0-4.8=11.2$. To have three figures, it should therefore be carried to the first decimal place. Then as it is made up of two quantities, one subtracted from the other, each of these should be carried (page xiv) to the decimal place desired in the result, i.e. to the tenths' place. This requires $D^{2}$ to contain three figures, 16.0 , and hence $D$ should contain three figures (since $D^{2}$ consists of the factors $D \times D$ ), i.e. should be written 4.03 inches. The requirement of one decimal place in $d^{2}$ calls for but two figures, 4.8 , and hence two figures, 2.2 inches, in $d$. The numerical expression to be solved would then be

$$
c=110000 \frac{3 \cdot 14\left(4.03^{2}-2.2^{2}\right)}{4}
$$

which would be most easily worked by a simple slide rule, or by direct multipllcation.

Example 11. - Desired to o. I per cent the fraction of dry steam in a sample of steam, using the following observations made with the "Barrus Calorimeter" (Peabody's "Themodynamics of the Steam Engine," page 234), the formula being

where

$$
x=\frac{W\left(q_{2}-q_{1}\right)+e-v\left(q-q_{3}\right)}{v r},
$$

$x=$ fraction of a mixture which is dry steam. . . . .

$W=$ weight of cooling water . . . . . . . 5735 lbs.

$20=$ weight of condensed water . . . . . . . $29.89 \mathrm{lbs}$.

$t=$ temperature of steam . . . . . . . . . .

$t_{1}=$ initial temperature of cooling water . . . . . $37^{\circ} .49 \mathrm{~F}$.

$t_{2}=$ final temperature of cooling water . . . . . . $83^{\circ} .84 \mathrm{~F}$.

$t_{3}=$ temperature of condensed water . . . . $304^{\circ} .88 \mathrm{~F}$.

$7_{33}=$ "heat of liquid" at $t^{\circ}$, from $t_{3}$ and tables . . . . . 274.4

$\mathscr{T}_{2}=$ "heat of liquid" at $t_{2}^{\circ}$, from $t_{2}$ and tables . . . . $\quad$ 51.91 B. T. U.

$t_{1}=$ "heat of liquid" at $t_{1}{ }_{1}$, from $t_{1}$ and tables . . . . 5.53 B. T. U.

$q=$ "heat of liquid" at $t^{\circ}$, from $t$ and tables . . . 287.6 B. T. U.

$e=$ radiation loss during test . . . . . . . . 120. B. T. U.

$r=$ latent heat of steam at $t^{\circ}$, from tables . . . . 891.2 B.T.U.

What number of places of flgures should be used throughout?

Solution.-For O.I per cent the result, and therefore all factors, should have five places of figures (page xiii). 'The only factors of the whole expression 
are the whole numerator, $v$, and $r$. They should therefore be carried to five places. Note, however, that $w$ and $r$ are not so given in the data. Whether, however, they are given closely enough must be determined by other means (see remark at foot of page $\mathbf{x x}$ ). Their product must, however, be carried to five places, and five-place log tables should be employed. The numerator consists of three terms, whose values, roughly, are

$$
\begin{aligned}
& 570(52 .-6 .)=2600 ., 120 ., \text { and } 30(288 .-274 .)=420 . \\
\therefore \quad & \text { numerator }=2600 .+120 .-420 .=2300 \text {., roughly. }
\end{aligned}
$$

To have five places the numerator must be carried to one place of decimals, as also must each of its terms. The first term is composed of two factors, $W$ and $\left(\eta_{2}-\eta_{1}\right)$, each of which must therefore be carried to five places. Then as $\eta_{2}-q_{1}=52 .-6=46$. roughly, it must be carried to the third decimal place. Hence $q_{2}$ and $q_{1}$ must each be carried to the third decimal place. The third term, in order to extend to the first decimal place, must cuntain four figures. It consists of two factors, $w$ and $\left(q-q_{3}\right)$, each of which must thus contain four figures. To make the value of $q-q_{3}$ contain four figures, its numerical value, 14 , must be carried to the second decimal place. This would require that both $q$ and $\eta_{3}$ be carried to the second decimal place, or to five fiprures each.

To summarize, then, putting in an interrngation point wherever a figure is wanting in the data, we have as the numerical expression to be solved

$$
x=\frac{573.5 ?(51.91 ?-5.53 ?)+120 . ?-29.89(287.6 ?-274.4 ?)}{29.89 ? \times 891.2 ?}=0.988 .
$$

Obviously, then, on inspection, although we might carry out the products $W\left(q_{2}-q_{1}\right), w\left(q-q_{3}\right)$, and $v r$, each to the necessary five figures, much doubt is cast upon the sufficiency of the data themselves to give the desired 0.1 jer cent in the result. The problem would reuguire a detailed study by other methods to decide that point, - the result of which, it may be incidentally asserted, would be adverse.

Note. - It may not be amiss in connection with these problems to call attention to the very large amount of engineering computations in which four, often three (slide rule), places of figures are abundant. In the design of machines and structures, the strength and sizes of parts, such as struts and tie rods, heams, pillars, shafting, etc., and the strains or stresses in them, often cannot, or need not, be fixed upon within an accuracy of one or even many per cent. This limit is fixed by the unreliability of materials or workmanship, or by ignorance of the exart conditions w which the parts may be subjected. like une.rtainties affect many of the data upon which specifications, estimates, and contracts for engineering work are based. and the experimental constants in sundry formulas. More than three or four places of figures can be imlulged in for sulh work only at an extravagant waste of time. On the other hand it is neecessary of discriminate sharply such operations as linear, angular, surface. and sometimes levelling, measurements in surveying and gendetie work where the accuracy may be very high. 


\section{LOGARITIIMS.}

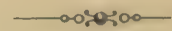

BEFORE reading the following pages become familiar with the "Notation by Powers of 10," page xv.

The common or Briggs logarithm of a given number is the exponent of that power to which ro must be raised to produce the number. Thus, 3 is the common logarithm of 1000 , since $10^{3}=1000$.

To multiply numbers together, add their logarithms. The sum is the logarithm of the desired product.

Proof. - The product $\mathrm{IO}^{a} \times \mathrm{IO}^{b} \times \cdots \times \mathrm{IO}^{m}$ is $\mathrm{IO}^{a+b \cdots+m}$, since powers of the same number may be multiplied together by adding their exponents. Therefore, if $A=10^{a}, B=10^{b}, \ldots, M=10^{m}$, $A \times B \times \cdots \times M=10^{a+b+\cdots+m}$. That is, $a+b+\cdots+m$ is the $\log -$ arithm of $A \times B \times \cdots \times M$. But $a, b, \cdots, m$ are respectively $\log A$, $\log \boldsymbol{B}, \cdots, \log \boldsymbol{M}$. Hence, $\log (A \times B \times \cdots \times M)=\log A+\log B$ $+\cdots+\log M$.

To divide one number by another, subtract the logarithm of the latter from that of the former. The difference is the logarithm of the quotient.

Proof. - Following the foregoing notation, $A / B=10^{a} / \mathrm{ro}^{6}=$ $\mathrm{ro}^{a} \times 10^{b}=10^{a-b}$. Hence $\log A / B=a-b=\log A-\log B$.

Logarithm of a number of several figures. - As $I=10^{0}$ and $10={ }^{1}$, the logarithm of $\mathrm{I}$ is $\mathrm{O}$ and of $\mathrm{IO}$ is $\mathrm{I}$, and the logarithm of any number greater than o and less than 10 , that is, of any number in the units' place (whether or not followed by a decimal fraction) is less than $\mathrm{I}$, that is, it is a fraction. It is expressed as a decimal fraction.

From the definition of a logarithm it is obvious that the logarithm of any stated power of 10 is the index of the power; i.e. $\log 1 \mathrm{r}^{ \pm n}$ $= \pm n$ when $\pm n$ is any number, whole or fractional, positive or negxxiv 
ative. Hence, taking first a specific example, the logarithm of 306.2, being of course the same as the logarithm of its equal, is the same as the logarithm of $3.062 \cdot 10^{2}$ (see "Notation by Powers of 10 ," page $\mathrm{xv}$ ), which is, $\log 3.062+\log 10^{2}=.4860+2$. , which is usually written 2.4860. The 4860 is found from tables, as shown later.

Similarly, $\log 0.003062=\log 3.062 \cdot 10^{-3}=\log 3.062+\log 10^{-3}=$ $.4860-3 .$, which is usually written either $\overline{3} .4860$ or $7.4860-10$, as will be further explained.

The logarithm then consists of, or may be separated into, two parts, viz. first, the decimal part called the mantissa, which is the logarithm of the first factor in the above separation; second, the integral part, or whole number, preceding the decimal point, and called the characteristic or index, which is the logarithm of the second factor $10^{ \pm n}$, and which, therefore, is $\pm n$.

Tables of logarithms contain the logarithms of the numbers from I. to ro., by steps larger or smaller, and to as many decimal places as may be recuuisite for the accuracy sought in the work in which they are to be employed. But all numbers whatever, from o to $\infty$, are one of these numbers $x$. to ro. multiplied by some power of ten, i.e. by roะn. For example,

$$
4628326=4.628326 \cdot 10^{6} \text {, and } 0.03986=3.986 \cdot 10^{-2} \text {. }
$$

Hence, the tables enable one, by merely prefixing to the tabular value the proper "characteristic" $\pm n$, to obtain the logarithm of any number whatever, from zero to infinity. The quantity directly given in the table is obviously the mantissa of the desired logarithm, and is therefore always a decimal fraction.

Since any table gives the mantissa to only a specified number of decimal places, it ean represent only a correspondingly restricted number of places of significant figures in the original number. It is to be remembered that a change of one unit in the last decimal place of the mantissa corresponds at all points throughout a table to a constant percentage change ("Definitions and Explanations") in the number corresponding. The amount of this change is such that it becomes the proper custom to use logarithm tables giving the mantissa to a number of places equal to the number of significant figures retained in the original quantities. 'Thus, if the numbers entering into the computation are properly retained to four places of significant figures, a four-place logarithm table should be used in connection with them; if to five significant figures, a five-place table; and so on. 
Four-place logarithm tables contain the logarithms to four decimal places of all numbers of three tigures from 1.00 to 9.99 , and enable one by interpolation to ohtain the four-place logarithm of any fourplace number from 1.000 to 9.999 . By merely prefixing the proper characteristic $\pm n$, therefore, the four-place logarithm of any fourplace number from o to $\infty$ is obtained, or, in other words, the fourplace logarithm of any number whatever from o to $\infty$ in so far as this is governed by the first four significant figures of the number. Four-place tables should not be employed upon work of an accuracy exceeding one-half of one per cent.

Five-place tables give directly the logarithms to one place further than four-place tables, i.e. to five decimal places, for numbers from r.000 to 9.999 , and thence, by interpolation, from $\mathbf{r} .0000$ to 9.9999 . Thus, with the proper characteristic, these tables furnish the logarithms of all five-figure numbers from o to $\infty$, that is, of any number whatever in so far as this is governed by the first five significant figures of the number. Five-place tables should not be employed in work of an accuracy exceeding one-twentieth of one per cent.

Six-place tables are sometimes arranged with the same steps as five-place, i.e. giving directly the logarithm to six decimal places of numbers from r.000 to 9.999 only. Such tables are of no practical service; for it is entirely useless to employ six-place logarithms in work on five-place numbers, and interpolation for six-place numbers in tables of so large steps as this, besides being less reliable, is more laborious and annoying than is the use of the more bulky tables of smaller steps.

If six-place tables are desired, it is usual to employ, dropping the last place, tables which give directly seven-place logarithms of numbers from 1.0000 to 9.9999 with convenient interpolation tables for the next place. Of these, the Vega tables are among the most convenient, legible, and reliable, being also comparatively inexpensive. The seventh place is very rarely demanded by physical, chemical, or engineering work.

The relative labor in using four, five, and six place tables lies probably between the ratios $1: 2: 3$ and $2: 3: 4$. Assuming the first estimate to hold, the labor is doubled by using a five-place instead of a four-place table, and is increased one-half by using a six instead of a five place table. Hence, as there is no sensible gain from using an excess of places, it is obviously very important to employ a table of the smallest admissible number of places. But, on the other hand, the use of too few places must be guarded against. As an instance of a somewhat dangerous practice may be cited the use of four-place 
tables in 0.1 per cent work. This is a not infrequent practice, most common perhaps in chenical computation, and, of course, arising from the exceeding convenience of cards containing four-place logarithms. It will be shown at page $x$ liv, that four places are not suffieient for 0.1 per cent direct computations, and the error if four-place lugarithms are used is sensibly the same. The computation error may easily rise to 0.2 or 0.3 per cent with four-place tables even in ordinary computations.

\section{To Find the Logarithms of a Number.}

RuLE. - Regard the number $\boldsymbol{Q}$ as separated into two factors $q \times 10^{ \pm n}$, where $q$ begins in the units' place (see "Notation by Powers of 10," page $x v$ ). Find in the tables the logarithm of $q$. This will be the mantissa of the desired logarithm. Prefix to this the characteristic or index $\pm \boldsymbol{n}$.

A few examples will sufficiently elucidate the process.

Example. - Desired the logarithm to four decimal places of the number 306.

Write the number, or, better, merely consider it as if factored in the form 3.06.102. In the four-place table, on the line 3.0 and in the column headed 6 will be found .4857 , which is $\log 3.06$. Obviously, $\log 10^{2}=2$. Therefore $\log 306 .=\log 3.06+\log 10^{2}=.4857+2$, which is usually written 2.4857 .

Further Examples. Interpolation. - Desired to four decimal places the logarithm of 306.2 .

This will lie between the logs of 306 and 307 (and approximately 0.2 of the way), and as the table is not carried out further we must interpolate.

\begin{tabular}{|c|c|c|}
\hline For 3.07 we find & .4871 & Difference $=.0014$, usually written \\
\hline For 3.06 we find & 4857 & r4. \\
\hline $\begin{array}{r}\text { Interpolation, } 0.2 \text { of } 14=2.8= \\
\therefore \log 3.062= \\
\log 306.2=\log 3.062+\log 10^{2}=\end{array}$ & $\frac{3}{4860}$ & $\begin{array}{l}\text { The desired mantissa will be } 0.2 \text { of } \\
\text { the way from } .4857 \text { to } .4871 \text {. Hence } \\
\text { we must add to the former number } \\
0.2 \cdot 14=3 \text {. }\end{array}$ \\
\hline
\end{tabular}

The interpolation may always be made by subtracting and multiplying as in this example, but to save the labor, logarithm tables are usually provided with marginal interpolation tables, by the aid of which interpolations may easily be made mentally.

Thus in taking out $\log 3.062$ we find $\log 3.06=.4857$. By inspection, difference $=14$. In the interpulation table healed 14 , line 2 , stands 3 , which is therefore the desired 0.2 of 14 . Therefore $\log 3.062=.4860$. This operation could, of course, be carried out mentally.

The present tables are arranged so that the intorpolation tables stand oppogite, or nearly so, to the logarithms to which they correspond. This not only gives them a convenient location but enables the computer usually on avoid even the mental subtraction of the successive longarithms to flnd the difference, since this will, of course, be that at the head of the nearest difference table. I:sually also the error introduced by using an interpolation table slightly wo large or too small is negligible. 
Interpolation becomes more laborious and more liable to error, if conducted mentally, in proportion as the difference is large. It is therefore greatest in the first quarter of any logarithm table. But it happens that in physical, chemical, and engineering computations there very often enter correction or reduction factors and other terms of the form $(\mathrm{I}+a)$ or $\mathbf{I} /(\mathbf{I}-a)$, where $a$ is a decimal fraction rarely as large as o.I. The frequency of such terms calls for a disproportionately large number of the more laborious interpolations. To avoid this labor and increased chance of error, the excellent practice has been adopted in some five-place tables of inserting two pages giving the logarithms from r.o to r.I, by steps only onetenth as large as in the rest of the tables, thus doing away with all interpolation in this most used and most troublesome portion of the table, without adding seriously to its bulk. Such a table has here been prefixed to the five-place table. In the four-place table the same result has been accomplished here, in a manner which is perhaps novel, by the insertion of ten additional lines at the head of the table. The Vega seven-place tables unfortunately lack this feature.

To find Logarithm of a Decimal Fraction. - The procedure is precisely the same as for a whole number. Note that the logarithm of a decimal fraction is always negative, and, conversely, that a negative characteristic always denotes a decimal fraction.

Example. - Desired the log of 0.003062.

$$
\begin{aligned}
\log 0.003062 & =\log 3.062^{\circ} 10^{-8} \\
\log 3.07 & =.4871 \\
3.06 & =.4857 \mid \text { Difference = I4. } \\
0.2 \text { of difference } & =3 \\
\therefore \log 3.062 & =.4860 \\
\log 10^{-8} & =-3 . \\
\therefore \log 0.003062 & =.4860-3 .
\end{aligned}
$$

This is written either $\overline{3} .4860$ or $7 \cdot 4860$ - 10. The latter form is obtained by adding ro to the characteristic and appending - ro to the whole. The numbers thus appended must in many instances, but not in all, be followed up in the computation in order to correctly locate the decimal point at the close. This is, however, usually very little trouble. The second method is the very general practice and is based on the assertion that when several logarithms are to be added, it is more convenient to have all the characteristics positive. 'The author is, however, of the opinion that this conventional method serves almost no useful purpose, and that it is better and less troublesome in every way to retain the negative characteristic. It is almost as easy to add a column of numbers in which some are negative, and are therefo:e subtracted when they are 
reached instead of being added, as to add a column in which all are positive. And if the negative characteristic is retained, all care and writing of -10 or its multiples is avoided. Moreover, the lingarithm is complete in itself and shows at once that it is the $\operatorname{lng}$ of a decimal fraction. These rewarks apply not only to the logarithms of ordinary numbers but to the logarithms of the trigonometric functions.

Example of addition where some of the characteristics are negative. Places beyond the first of the mantissa are not added in this illustration.

2.4036 Beginning at the bottom of the columns we have

T.2168

1. 3462

$\overline{2} .5113$

I. 4

$$
\begin{aligned}
& 5+3=\mathbf{8}+2=\mathbf{1 0}+4=\mathbf{1 4}, \text { write } 4 ; \\
& \text { carry } \mathbf{I}+\overline{\mathbf{Z}}=\overline{\mathbf{I}}+\mathbf{I}=\mathbf{0}+\overline{\mathbf{I}}=\overline{\mathbf{I}}+2=\mathbf{1} .
\end{aligned}
$$

Of course the figures printed in heavy type are the only ones pronounced or mentally enforced.

Examples of some cases where the use of the notation by powers of to enable one to dispense with the characteristic in portions of computations are given incidentally at page xxi.

Grouping by Fives in the Tables. - Throughout the entire set of tables, it will be observed, the columns and lines are arranged in groups of five. This not only aids the eye to readily follow any desired line or column, but enables the computer with a little practice to enter a desired column or line without glancing at the number at the top of the column or side of the line, and similarly to read off the number of column or line without glancing off to the marginal figure. Thus the middle column in the second group is 7 , the last in the first group is 4 , and so on. The practice of working by observed position rather than by the marginal number should be pursued. It reduces fatigue and tends to prevent mistakes.

Indexing at Corners of Tables. - The bold type at the outer corners of the tables shows the contents of the page and its opposite. Thus on the sixth page of the five-place log table, the larger black figure 2 shows that these two pages contain the logs of all the numbers beginning with 2. The smaller black figures, $30, \mathbf{4 7}$, in the margin show that the mantissa on the two pages extend from 30 to 47 (first two figures). The indexing will be found a material help in rapidly running the page corners under the finger to find either a desired table or a desired figure in a table.

Decimal Point in Logarithm Tables. - It is the almist universal custom to omit the decimal point entirely from lugarithm tables. This tends toward compactness, but is by no means essential on that score. The omission causes no serious inconvenience after slight. 
practice. On the other hand, the retention of the point renders the table complete and strictly self-consistent; that is, any mantissa in the table is then the completely expressed logarithin of the corresponding tabular number. This fact tends materially toward clear comprehension on the part of the beginner or occasional computer. The table is also then perfectly assimilated to the "Notation by Powers of 10," page xv, which affords not only the elearest basis of explanation of mantissa and characteristic, but by far the easiest method of obtaining and following the characteristic in computations. The points do not add necessarily to the bulk of the tables and are no hindrance to their use by computers accustomed to other rules than those here given respecting the characteristic. They are therefore retained in these tables.

Antilogarithm = Number Corresponding. - Given the logarithm to find the "number corresponding," i.e. the number of which this is the logarithm. This number is also called the "antilogarithm."

\section{From the Log Tables. -}

Example. - To find the antilog of 2.4857 , inspect the body of the four-place $\log$ table to find the mantissa .4857 (or the next smaller than this if the exact value does not appear). It will be found to lie on line 3.0 and in column 6 , and is, hence, the $\log$ of 3.06. The characteristic 2 is the $\log$ of $\mathrm{xO}^{2}$.

$\therefore$ antilog $2.4857=3.06 \cdot 10^{2}$ or 306 .

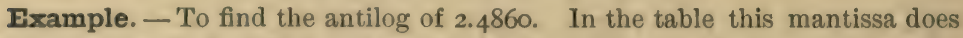
not appear exactly, but the next smaller is .4857 , whose antilog is 3.06 , and the difference from this to the next larger is $\mathbf{I}$.

$$
\begin{aligned}
& .4860-.4857=3 \text {, and } \frac{3}{14}=.2, \\
\therefore \text { antilog } .4860 \text { is } \frac{3}{14} \text { or } 0.2 \text { of the way from } 3.06 \text { to } 3.07, & \\
\therefore \text { antilog } .4860 & =3.062, \\
& \text { antilog } 2 . \quad=10^{2},
\end{aligned}
$$

The interpolation can be mentally made by the marginal interpolation tables. The tabular difference is $\mathbf{1 4}$, and it is desired to know what decimal fraction the difference 3 is of this. Looking down the column under 14 for the number nearest to 3 it is found to be 3 and to stand opposite to 2. $\therefore 3$ is 0.2 of 14 , and antilog $\cdot 4860=3.062$.

It will be noticed that in the four-place and five-place log tables those mantissæ have been printed in heavier type in which the first figure changes from one digit to the next. This serves as a guide to the eye in looking for any desired mantissa whose antilog is sought.

From Tables of Antilogarithms. - Some computers prefer to employ a special table for antilogarithms instead of working backward in the ordinary logarithm table as in the preceding example. Whether 
the small saving in time effected by this means is an equivalent to the slight additional mental effort incident to the employment in alternation of tables differently arranged, may be questioned. Where many antilogs are to be taken out in succession, the gain is, however, sensible.

Example. - To find by the table of $A$ ntilogarithms the antilog 2.4857 .

Taking the mantissa first, we find on line .48 , column 5 , the next smaller number 3.055 with a tabular difference of 7 . Hence, antilog $.485=3.055$. But antilug .4857 will be 0.7 of the way from that of .485 to.486. The amount to be added then for interpolation is $0.7 \times 7=5$.

$$
\begin{aligned}
\text { antilog } .4857=3.055+0.005 & =3.060, \\
\text { antilog } 2.4857=3.060 \cdot 10^{2} & =306.0 .
\end{aligned}
$$
obvious.

Interpolation tables are provided whose method of use is sufficiently

Cologarithms = Logarithms of Reciprocals. - The colog of any number $p$ is the logarithm of the reciproeal of the number.

It is therefore $\quad \log \mathrm{I}-\log p=0-\log p$.

In substitution in a formula such as $x=\frac{a \cdot b \ldots}{c \cdot d \cdot e \ldots}$, which is the product of several numbers (one or more) divided by the product of several others (one or more), the direct process would be to take the sum of the logarithms of the facturs in the numerator, and to subtract from this the sum of the logs of the fretors in the denominator.

$$
\begin{aligned}
& \text { Example. - } \quad \log a=1.2543 \\
& \log b=\overline{3} .8766 \quad \log d=\overline{5} .2121 \\
& \log 8=2.1345 \\
& \text { Numerator sum }=\overline{\mathbf{T}} \cdot \mathbf{r} 309 \\
& \text { Denominator sum }=\overline{\mathbf{1} .2108} \\
& \text { Subtract denominator } 8 u m=1.2108 \\
& \text { Difference }=\overline{3} .9201 \\
& x=\text { number corresponding }=8.320 \cdot 10^{-3} \text {, or } 0.008320 \text {. }
\end{aligned}
$$

This process may be simplified by employing the colng. It then becomes

$$
\begin{aligned}
\log a & =1.2543 \\
\log b & =\overline{3} .8766 \\
\operatorname{colog} e & =\overline{4} \cdot 1358 \\
\operatorname{colog} d & =4.7879 \\
\operatorname{col} e & =\overline{3} .8655 \\
\operatorname{Sum} & =\overline{3} .9201 \\
x=\text { number corresponding } & =\mathbf{8} .320 \cdot 10^{-8}
\end{aligned}
$$

This procegs is thus reduced to the simple aldition of a serieg of numbers.

The colog may be easily taken out of the usual log table by merely looking out the logarithm and sulitracting mentally from 0 . 
Example. - Desired the colog of 306.2. $\log 306.2=2.4860, \operatorname{colog}=\overline{3} .5140$.

The use of cologs either with or without a table effects but small saving, except in case a series of substitutions in a given formula are to be made, so that a number of cologs may be looked out in immediate succession.

Table of Cologs. - The table of four-place cologarithms is arranged similarly in every respect to the table of four-place logarithms, and the cologs are taken from it just as logs from their table; noting, however, that the cologs in the table have the characteristic $\overline{\mathbf{I}}$, and that the differences are subtractive.

Example. - Desired the colog of 306.2. Separate into $3.062 \cdot 10^{2}$.

In line 3.0 , column 6 , colog 3.06

$0.2 \times(-14)$ by difference table

$\therefore \operatorname{colog} 3.062$

$\operatorname{colog} 10^{2}$

$=\overline{\mathbf{r}} \cdot 5143 \mid$ Difference from

$\therefore \operatorname{colog} 306.2=\operatorname{colog} 3.062^{\circ}+\operatorname{colog} 10^{2}$

$=-3 \mid \operatorname{colog} 3.07$ is -14 .

$=\overline{\overline{\mathrm{I}} \cdot 5140}$

$=\overline{2}$.

$=\overline{3.5140}$

Example.-Desired the colog of 0.007136.

In line 7.I, column 3 , is colog 7.13

$0.6(-6)$ by difference table

$$
\begin{array}{rr}
= & \overline{\mathrm{r}} .1469 \\
= & -4
\end{array} \mid \text { Difference is }-6 .
$$

$\operatorname{colog} 7.136$

colog $10^{-8}$

$=\overline{\mathbf{I}} \cdot 1465$

$\operatorname{colog} 0.007136=\operatorname{colog} 7.136+\operatorname{colog} 10^{-8}=\overline{2.1465}$

Habit in Reading off Numbers or Logarithms. - Time can be economized, strain on the attention reduced, and liability to mistake lessened by an easily acquired habit of grouping and emphasizing the figures in reading off the numbers, the mantissa, and the numbers corresponding (antilogs) in using tables.

A good method of reading is as follows:

In reading a number or antilog pay no regard to the decimal point. Emphasize the first figure; pause, read second and third figures; pause, read remaining figures in groups of three. Thus: Desired the $\log$ of 30.620472 . Read this as 30620472 , i.e. three ... naught six ... two naught four ... seven two.

In reading off a mantissa use no emphasis, but group the first two figures together, and the subsequent figures by threes. Thus, the mantissa .4869 would be read as 4869 , i.e. forty-eight . . sixtynine; and .4860r as 4860 r, i.e. forty-eight ... six naught one.

In taking from the log table the numbers corresponding (antilog) to .48601 , it would be read 3062 , three ... naught six . . . two, precisely as under the above rule for reading a number. 
These rules apply equally well to four or five place tables. In tables of six or seven places the first three figures of the mantissa are grouped together in printing, and are therefore more conveniently read off together. Also, it is more convenient to read off six-place numbers and antilogs with three figures instead of two in the second group; thus, 781462 .

The difference between a number and a mantissa thus read off, whether audibly or mentally, almost precludes the possibility of mistaking one for the other, so that less strict attention will be required to aroid entering the number column of a table with a mantissa, or vice versa. It also avoids the mental or verbal employment of words of instruction. Thus, if a computer reads off 782 he knows, or his assistant using the log tables knows, as soon as the first figure has been read that the logarithm of the number is desired. Conversely, if he reads off 43857 , the first two figures alone show that the quantity is a mantissa, and that the antilog is required. 'Thus, no words of instruction need be used throughout an entire computation, and yet no possibility of error need enter.

It is also to be noted that this grouping is consistent with the symmetrical grouping advocated at an earlier page ; adapts itself perfectly to the employment of the notation by powers of 10 ; and coincides with the most convenient grouping in the five-place tables.

Powers and Roots by Logarithms. - If $a=10^{m}$ (so that $m=\log a$ ) then $(a)^{n}=\left(10^{m}\right)^{n}$ and $\log \left(a^{n}\right)=n \log a$. Here both $m$ and $n$ may be either positive or negative, and either an integer or a fraction.

Hence, to raise any number, whole or fractional, to any power, integral or fractional, and positive or negative, multiply the logarithm of the number by the exponent of the power of the number.

Since a root is a fractional power, i.e. $\sqrt[n]{a}=a^{n}$, the above rule includes the extraction of a root. In the case of decimal fractions the fact that the characteristic is negative while the mantissa is positive, must be regarded. The correct result will be assured if the mantissa and characteristic are separately multiplied (or divided) by the exponent, and the latter result then subtrated from the former, as will be further shown. Certain special procedures will also be deseribed.

The one exception to this is where the index of the power is a single flgure and positive. This case takes care of itself without squecial attention.

It may seem that the use of the negative characteristic thus increases labor over the usual notation explained at the font of page xxviii. Inspection of examples will show that the only case in which it does so is where the inclex of the power or rot is other than a single digit. 


\section{Powers and Roots of Numbers greater than Unity. -}

Framples. - Desired the cube of $47 \cdot 16$, i.e. $(47 \cdot 16)^{3}$.

$$
\begin{array}{ll}
\log 47 \cdot 16 & =\mathbf{1} 6735 \\
\text { Multiply by } & =\frac{3}{5.0205} \therefore x=1.048 \cdot 10^{5} \text {, or } 104800 . \\
\log x & \therefore x
\end{array}
$$

Desired $\sqrt[5]{471.6}$

$$
\begin{array}{ll}
\log 471.6 & =2.6735 \\
\text { Divide by } & \frac{5}{0.5347} \quad \therefore x=3.425 . \\
\log x & \therefore
\end{array}
$$

Example. - Desired the 2.416 power of 65830 .

$$
\begin{aligned}
\log 65830 .= & 4.8184 \\
\text { Multiply by } \quad & \frac{2.416}{96368} \\
& 192736 \\
& 4818 \\
\therefore \log \quad(65830)^{2.416}= & \frac{2892}{1.6413} \\
\therefore \quad(65830)^{2.416}= & 4.378 \cdot 10^{11}=437800000000 .
\end{aligned}
$$

To avoid the direct multiplication of the logarithm by the index of power, when this contains several figures, their logs may be taken. Thus

$$
\begin{array}{rlrl}
\log 2.416 & =0.38310 & & \frac{0.3831}{1.0660} \\
\log \log (65830)^{2.416} & =\frac{1.06601}{1.06} & & 11.64 \\
\text { antilog 1.06 601 }= & \log (65830)^{2.416} & =11.641 & \\
\therefore(65830)^{2.416} & =4.376 \cdot 10^{11} & 4.365^{\circ} 10^{11} .
\end{array}
$$

When the $\log$ of a $\log$ is thus taken, a table giving at least one place more in the mantissa should be used than would be otherwise needed in order to protect the last place of figures in the result, as is shown in the above example, which is by no means an extreme case. If the quantity is a decimal fraction, the negative characteristic must be separately treated and the result subtracted from the result obtained with the mantissa.

Powers of Decimal Fractions. - Here it is necessary to notice that the logarithm of a decimal fraction is composed of two parts, one positive the other negative. Thus,

$$
\log 0.0683 \mathrm{I}=\overline{\mathbf{2}} . \mathbf{8}_{345} \text { or } 8.8345-\text { ro., }
$$

according to the notation chosen. In the first form (the one here recommended) the logarithm consists of a negative characteristic 
and a positive mantissa ; in the second form it consists of a positive logarithm (both characteristic and mantissa) followed by a negative characteristic.

No new procedure is necessary, but it is essential to be consistent as to sign of each part, and to remember that both parts must be subjeeted to any operation performed upon either. Whenever the exponent las more than one figure, the negative characteristie and the positive mantissa should be separately multiplied by the index. and the former subtracted from the latter.

Example. - Desired (0.00 4716)4.

$$
\begin{array}{rlll}
\begin{array}{l}
\log 0.004716 \\
\text { Multiply by }
\end{array} & \overline{3} .6735 & \text { or } & 7.6735-10 \\
& \overline{10.6940} & \text { or } & \frac{4}{30.6940-40} \text {, or } 0.6940-10
\end{array}
$$

$\therefore(0.004716)^{4}=$ number corresponding, or antilog,

$$
=4.943 \cdot 10^{-10} \text { or } 0.0000000004943 \text {. }
$$

Example. - Desired $(0.004716)^{4.29}$.

$$
\begin{aligned}
& \log 0.004716=\overline{3} .6735 \text { or } \quad 7.6735-10 \\
& \frac{4.32}{26940} \quad \frac{4.324 .32}{306940-43.2} \\
& 20215 \quad 230205 \\
& \frac{1348}{2.9095} \quad \frac{15348}{33.14953} \\
& \frac{-12.96}{\overline{I 1} .9495} \frac{-43.2}{\overline{\Pi 1} .9495} \\
& \therefore(0.004716)^{488}=2.962^{\prime} 10^{-11} \text {. }
\end{aligned}
$$

Roots of Decimal Fractions. - To extract the root of a decimal fraction, divide separately the negative characteristic and the positive mantissa by the index of the desired root. Subtract the former quotient from the latter. The difference will be the logarithm of the desired root. In other words, treat the characteristic separate from the logarithm. 'This procelure is always the safest to adopt when there is any doubt in the mind of the eomputer.

Example. - Desired $\sqrt[3]{0.06831}$.

$$
\begin{aligned}
& \log 0.06831=\overline{2} .8345 \\
& \text { Jividing characteristio by } \quad 3=-\overline{0.6667} \\
& \text { Dividing mantisan by } \quad 3=0.2782 \\
& \therefore \log \sqrt[3]{0.06831}=7.6115 \\
& \sqrt[3]{0.068_{31}}=\text { number corresponding }=4.085 \cdot 10^{-1} \text {. }
\end{aligned}
$$


A method easily understood by inspection is this: Add to the negative characteristic of the logarithm a number equal to $m r$ where $r$ is the index of the desired root and $m$ is a whole number large enough to make $m r$ larger than or equal to the characteristic, in other words, large enough to extinguish the negative sign of the characteristic. Write this number with a negative sign after the logarithm. Then divide the whole by the index $r$. The quotient will be the logarithm of the desired root. Usually $m=\mathbf{I}$, i.e. $m r=r$.

Example. - Desired $\sqrt[8]{0.0683^{1}}$

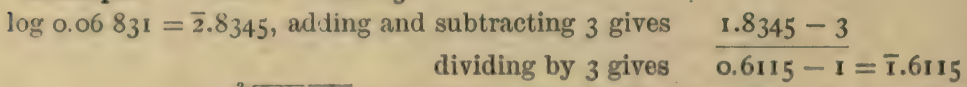
$\therefore \sqrt[3]{0.06} \overline{831}=$ number corresponding $=4.088 \cdot 10^{-1}$ or 0.4088 .

\section{SQUARES AND SQUARE ROOTS.}

It is common to give separate tables of squares and of square roots by which, respectively, the square or square root of any number may be taken out. Inspection, however, shows that the fourplace table of square roots must occupy four pages, the first two containing the numbers from r.o to ro.; the second two from ro. to roo.; the corresponding roots ranging from 1 . to ro.; while a table of squares would occupy but two pages, containing numbers from r.o to ro., the squares ranging from r. to roo. Hence the tabular differences in the table of square roots will be much smaller than in the table of squares. For this reason the table of squares may advantageously be dispensed with, and squares be taken out when desired from the table of square roots, as the numbers corresponding (antilogarithms) are taken out of a table of logarithms. The smallness of the differences makes interpolation easier and more rapid than in a table of squares, and this more than offsets the slight disadvantage of the reverse process of interpolation. The tables at page 30 give square roots to four places. As in the logarithm tables, the numbers in the body of the table are printed in stronger type wherever the second figure changes, in order to assist the eye in the reverse use of the table.

In the table of square roots the insertion of the extra section giving 1.00 to 1. 10 to four places direct to avoid interpolation is not called for as in logarithms because the interpolation is very easy, and because the squares of terms of the form $(\mathrm{I}+\alpha)$ are not frequently required. 
To find the square of any number, factor the number as described in the "Notation by Powers of ro," page xy. Enter the body of the table with the first factor, and find the corresponding marginal reading and column healing which will be the first three figures of the square. Interpolate for the fourth figure. Square the factor $10^{\prime \prime}$, which makes it $10^{\text {tan }}$. Multiply together these two squares.

Example. - Desired the square of 34850 .

$$
34850.0^{2}=\left(3.485 \cdot 10^{4}\right)^{2}=3.485^{2 \cdot 10^{2 \times 4}} \text {. }
$$

In the table 3.479 (the next smallest value to 3.485 ) stands in line 12 , column 1. The tabular difference is $\mathbf{1} 4$, of which our difference $(=3.485-3.479=6)$ is $f^{6}$, or 0.4 (as may be seen at once from the interpolation table heacled 14).

Hence

$$
34^{850} .^{2}=12.14^{\cdot 10^{8}}=121400000 .
$$

Example.-Desired $(0.0004983)^{2}$.

$$
(0.0004981)^{2}=4.981^{2} \cdot 10^{-4 \times 2}=24.83 \cdot 10^{-8}=0.0000002483 \text {. }
$$

To find the square root of any number, factor it as in the "Notation by luwers of $10, "$ page $\mathrm{xv}$, except that $n$ must be now an even number, while the first factor must have either one or two digits preceding the decimal point, whichever may be necessary, in order to permit $n$ to be made even.

Find, then, from the table, interpolating if necessary, the square root of the first factor, and multiply this by $10^{: m / 2}$, the square root of the second factor; the product is the desired square root.

Example. - Desired the square root of 347.6 .

$$
(347.6)^{\frac{1}{2}}=\left(3.476 \cdot 10^{2}\right)^{\frac{1}{2}} \text {. }
$$

In the tahle, line 3.4 , eolumn 7 , gives $(3.47)^{\frac{b}{2}}=1.863$

Interpolations by table gives 0.6 difference $=\mathrm{I}$

$$
\begin{aligned}
&(3.476)^{\frac{d}{2}}=\overline{1.864} \\
& \therefore(347.6)^{\frac{1}{2}}=1.864 \cdot 10^{1}=18.64
\end{aligned}
$$

Example. - Desired $\sqrt{875200}$

$$
\sqrt{87 \cdot 52 \cdot 10^{4}}=9 \cdot 355^{\circ} 10^{2}=935 \cdot 5 \text {. }
$$

Example. - Desired $(0.05643)^{\frac{1}{2}}$

$$
\left(5.643^{\circ} \times 0^{-3}\right)^{1}=2.376 \cdot 10^{-1}=0.2376
$$

Example. - Desired 0,00006 784.

$$
67.84 \cdot 10^{-6}=8.236 \cdot 10^{-3}=0.008236
$$




\section{RECIPROCALS.}

This table, page 34 , contains the reciprocals to four places of numbers from 1 . to 9.99, and by interpolation from 1 . to 9.999. The reciproeal to four places of any number from zero to infinity is, of course, one of these tabular values multiplied by a suitable power of $\mathbf{~} \mathbf{0}$. In this table, the reciprocals of numbers from $\mathbf{r}$. to 1.100 are given directly in the first ten lines to avoid interpolation in finding the reciprocals of terms of the form $(\mathbf{I}+a)$, where $a$ is a small decimal fraction.

N.B. - The approximation $1 /(1+a)=(1-a)$ approx., is frequently employed in computations to avoid dividing by $(1+a)$. This useful approximation, however, must be used with caution. The error from its employment is $a^{2}$; that is, if $a=0.1$, the error from multiplying by $(1-a)$ instead of dividing by $(1+a)$ is $0.1^{2}=0.01$, or $I$ per cent; if $a=0.03$, the error is $0.03^{2}$ $=0.0009$, or nearly o. 1 per cent, and so on.

To find the reciprocal of a number, factor it by the "Notation by Powers of ro," page xv. Find the reciprocal of the first factor by the table and multiply this by the second factor $10^{ \pm n}$ with the algebraic sign of its exponent reversed, i.e. by $10^{\mp n}$.

Example.-Desired 1/4486, i.e. $(4486)^{-1}$.

$$
(4486 .)^{-1}=\left(4.486 \cdot 10^{8}\right)^{-1}=(4 \cdot 486 .)^{-1} \cdot \mathrm{IO}^{-8} .
$$

In table, line 4.4 , col. 8 , By difference table, $(4.48)^{-1}=0.2232$

Reciprocal of 0.6 difference $=-3$

$$
\begin{aligned}
4.486 & =\overline{0.2229} \\
(4486 .)^{-1}=(4.486)^{-1} \cdot 10^{-8} & =0.2229 \cdot 10^{-8}=0.0002229 .
\end{aligned}
$$

Example. - Desired I/0.00 3275 .

$$
(0.003275)^{-1}=(3.275)^{-1} \cdot\left(10^{-8}\right)^{-1}=0.3054 \cdot 10^{3}=305.4 .
$$

\section{NATURAL SINES, COSINES, TANGENTS, AND COTANGENTS.}

It is frequently convenient to have a table giving rough values of the natural trigonometric functions for use in preliminary, check, or approximate computations. The four-place tables of the above four functions cover all ordinary needs. Interpolation can be made in these tables to $0^{\circ}$.or by the interpolation tables, or to $\mathbf{I}^{\prime}$ by mental computation, since the step between successive columns is $0^{\circ} . x$, or 6 '. 
Example. - Desired the natural sine of $37^{\circ} \cdot 75^{\circ}$

On line $37^{\circ}$ under heading ${ }^{\circ} .7$ is 0.6115 . To interpolate for the remaining figure we must add 0.5 of the tabular difference, which, by inspection, is 14 . $0.5 \times 14=7$, as can be seen at once in the interpolation table 14 opposite the line $37^{\circ}$.

$$
\therefore \sin 37^{\circ} \cdot 75=0.6115+.0007=0.6122 \text {. }
$$

Example. - Desired the natural cotangent of $72^{\circ} \cdot 28$.

The cosines and cotangents read upward in the right-hand degree column.

$$
\begin{array}{lr}
\text { Line } 72^{\circ} \text {, column } 0.2 \text {, gives } & \begin{array}{r}
0.3211 \\
0.8 \text { of difference }(19) \text {, to be subtracted, } \\
\therefore \text { natural cotangent } 72^{\circ} .28
\end{array}=\frac{-15}{=0.3196}
\end{array}
$$

All of the tables are used similarly.

\section{LOGARITHMS OF SINES, COSINES, TANGENTS, AND COTANGENTS.}

I'wo sets of tables are given, four-place and five-plaue, respectively. The four-place tables read to tenths and hundredths of degrees, and are convenient for general rough work and with instruments reading to tenths of a degree. The five-place tables give values to minutes direct. They are adapted to a very large part of the angular measurements ordinarily made in experimental work. Interpolation to half or quarter minutes is easily male in them mentally. A computer working clnser than that would naturally employ the Vega, or other conveniently arranged six-or seven-place tables, dropping unnecessary places.

For the reasons stated under the discussion of logarithu taliles, fage $x$ xviii, the negative charateristie is here retained insteal of the more customary 9, with the appended - ro.

The use of the tables nesds little explanation. The four-place tables are used precisely as the tables of the natural fumetions. In the five-place tables, for less than $45^{\circ}$, real the tahles downwards. using the minute column at the left hand. For angles greater than $45^{\circ}$, real the tables upwaris, using the minute column at the right. hand of the table, and take eare to employ the column-headings given at the foot of the table.

\section{Examples. -}

I.og $\sin 31^{\circ} 22^{\prime}=\overline{1} .78643$

$\log \tan 54^{\circ} 46^{\prime}=0.15101$. 


\section{SLIDE WIRE RATIOS.}

The increasing use of the slide wire in electrical measurements, notably in connection with physical chemistry, renders the table of values of

$$
\frac{a}{1000-a}
$$

a decided convenience. The work to which the slide wire is thus for the most part employed demands but four-place tables.

To use the table, let $a$ be the reading in millimetres on the slide wire, which is supposed to be one metre long with millimetre subdivision, or of any other length with division into thousandths. Find the first two figures of $a$ in the first column, and run out on this line to the column headed with the third figure. The number there found will give the value of the above fraction, which is the ratio of the length of one section of the wire to the other. If $a$ contains a fourth figure, that is, if read to tenths of a millimetre, interpolation must be made in the usual manner.

Example. - The slider reads 415.6 millimetres.

In line $4 \mathrm{I}$ under 5 is found 0.7094 . Interpolating by adding 0.6 of the tabular difference 29 , gives

$$
\begin{aligned}
x & =0.7094+0.6 \times 29 \\
& =0.7094+.0017=0.7111 .
\end{aligned}
$$




\title{
DEFINITIONS AND EXPLANATIONS
}

\author{
UNDERLYING THE COMPLTATION RULES.
}

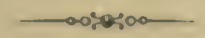

The following statements call for the attention of those only who find unfamilar terms in the foregoing rules.

A Digit is any one of the ten characters $1,2,3,4,5,6,7,8,9$, o.

A Significant Figure is any digit used to denote or signify the amount of the quantity in the place in which it stands. Thus zero may be a significant figure when it is written not merely to locate the decimal point, but $w$ indicate that the quantity in the place in which it stands is known to be nearer to zero than to any other digit.

For example, if a distance has been measured to the nearest fiftieth of an inch and found to be 205.46 inches, all five of the figures, including the zero, are signifieant. And similarly if the measurement had shown the distance to be nearer to 205.40 than to 205.41 or to 205.39 the final zero would be also significant, and should incariably be retained, since its presence serves the most useful purpose of showing that this place of figures had been measured as well as the rest. If in such a case the quantity had been written 205.4 instead of 205.40 , the inference would be drawn either that the hundredths of an inch had not been measured, or that the person who wrote the number was ignorant or careless of the proper numerical usage. Failure to follow this simple rule is a common source of annoyance and uncertainty.

A zero when used merely to enable the decimal point to be retained is of course not a significant figure in the above sense. $\boldsymbol{k}$. $l$. If the distance were measured as 286 . centimetres within \pm 1 centimetre, it might be retained as 2.86 metres, or 0.00286 kilometres, or 2560 . millimetres. In this case neither of the zeros would be significant.

From this last example it is obvious that when the first zero, or zeros, precede the decimal point, they fail to indicate intrinsically whether or not they are gignificant. E.g. The number $2 \$ 60$. millimetres or $2 \$ 600$, millimetres, standing apart from explanatory context, would afford no clew to whether the last place. or the last fwo places, had been measured. In writing such a number, therefore, whenever it is desirable to convey this information some statement of the reliability of the quantity must be appended. This is usually done by writing after the number th a where a is a number, of two significant figures only, representing the estimated measure of the accuraey or unreliability of the result. (Seve later.)

Places of Figures are the places in which figures stand in the number as artually written. Places of signifiean figures are these in which significant figures stand. These two terms being merely, although not actually, identical, are often used interchangeably. 
Example. -426 or8. has six places of figures and of significant figures 3479100 . has seven places of figures, but whether its number of places of significant figures is more than five is indeterminate. 0.027680 has seven places of figures with five places of significant figures. $2.7680^{1} 10^{-2}$ has tive places of figures and five significant figures.

Places of Decimals. - These, of course, are the places following the decimal point as the number happens to be written. E.y. 0.02768 o has six decimal places. 2. $76 \mathrm{So}^{10^{-2}}$ has four decimal places, although its magnitude is the same as that of the preceding quantity.

From the foregoing six examples it will be seen that the number of decimal places and the number of places of significant figures have no necessary mutual relation.

Accuracy; Reliability. - To know the exact accuracy of a given quantity it would be necessary, of course, to know the true value of that quantity. In the case of a few mathematical constants (such as $\pi=3.14$ I59 265 …) the true value is known, at least far beyond ordinary requirements. But in case of all measurements the same is obviously not true, for if the true value were known, measurements would be unnecessary. Some approximate expression of the accuracy of a measured result can, however, usually be obtained, and is necessary. Sometimes this is afforded by a knowledge of the instrument used and the degree of care employed. Thus, suppose the distance of about 3 feet $6{ }_{6}^{3}$ inches $=3.5039$ feet between two marks to have been carefully once measured with a good foot-rule, it could safely be assumed from our previous knowledge of such work that if a series of these measurements were undertaken, the results would vary from their mean by less than $\pm \frac{1}{32}$ of an inch; also, that the error in the rule itself, and from other unavoidable or unavoided sources, would not on the average materially increase the error of measurement. Then $\pm \frac{1}{32}$ inch $= \pm 0.0026 \mathrm{ft}$. would be taken as the estimated measure of accuracy of the result. Instead of expressing the accuracy in units, e.g. inches or feet, it is usually more convenient or intelligible to express it as a fraction; or, better still, in percentage. Thus, the foregoing will be

$$
\pm \frac{0.0026}{3 \cdot 5}= \pm 0.00074 \text {, i.e. } \frac{74}{100000} \text {, or } 100\left( \pm \frac{0.0026}{3.5}\right)= \pm 0.074 \text { per cent. }
$$

If in this example the reliability had not been estimated at $\frac{1}{32}$ inch, but if several measurements had been made, and these had been found upon inspection to deviate from their mean by about $\frac{1}{3} \Sigma$ of an inch, then, other things being the same, the measure of accuracy of any single measurement taken without knowledge of the others would be regarded as $\pm 3^{1}$ inch, or \pm 0.074 per cent.

Mean; Average; Deviation Measure. - When the result is the arithmetical mean or average of several separate measurements of the same quantity, its reliability or accuracy is taken to be in proportion to the square root of the number of such observations. Thus, in the last preceding example, if there had been $n=9$ single observations made, the measure of accuracy of the mean of these would be

$$
\pm \frac{1}{32} \div \sqrt{n}= \pm \frac{1}{3^{2}} \frac{I}{\sqrt{9}}= \pm \frac{1}{96}= \pm 0.010 \text { inoh, or } \pm 0.025 \text { per cent. }
$$

The differences of the single observations taken under like conditions from their mean will be called deviations, and their numerical ayerage (i.e. their sum 
omitting their algebraic sign, divided by their nun:ber) will be called the average deviation of the single observation from the mean, and will be denoted by ad. This quantity divided by the siguare root of $n$ would be called the average deviation of the mean, and will be denoted by $A D$. It is the averagre amount by which any one such mean would be found to deviate from the average of a number of such means all taken under like conditions. The term deciation metsure will be used in referring to either of these quantities.

Brietly, then, the meaning of the terms may be summarized as follows: By the statement that the accurcicy or relialility of a result is of a yuoted amount is meant that the deviation measure of this result is estimated or found to be of this stated amount, and that so far as this can be discovered by inspection, no other stourees of error exist which affect the result by an amount sensible as compared with this.*

It is essential to bear in mind in connection with all quantities that are the result of measurement that no absolute numerical expression of the accuracy or error is possible; that any expression which is given is usually merely a deviation measure; that is, an approximate average value of the effect of the variable parts of the errors, accompanied by an assurance, expressed or implied, that a study of the discoverable suurces of error of the process has been made, and that these have been corrected for or found to be negligible compared to the deviation measure.

In specifying the accuracy desired in the result, it must be understood that merely the converse of this is meant; or, at least, that only the converse is possible of attainment.

Rules for Significant Figures. - The rules are so framed that, barring mistakes, the greatest prossible computation error entering into the result of any ordinary computation (e.g. one involving a total of not much exceeding 20 component numbers, steps, or operations, where a rejection error may occur) ghall not be sensible compared with the errors of the measurements or data, or shall not sensibly affect the accuracy of the result. They are, therefore, sufe rules in the worst possible cases. But in order to be so they are necessarily more than sufficiently stringent for some classes of comparatively rough work, where the infrequent undetected entrance of a computation error two to four times as large as the experimental error would be permisibible. For such work tone less place of figures may be used, but when the rules are thus relaxed the possible consequence should be borne in mind, and special scrutiny applied to the various stages of the computation, special attention being directed to quantities beginning with 1 or 2 .

Rejection Error. - Whenever it becomes necessary to throw off places of figures, a "rejection error" may enter into the result; e.g. suppose that for auy reason the last two figures are $\omega$ be rejected in

making it

24375291 ,

24375300 .

The rejertion error in the new form is evidently +9 .

In rejertion, the last figure relainel is always to be increased by tw when the rojected figure uext it is 5 or over, but remains unchanged if that figure is less than 5 .

- For a more extended diseruswion of this and allied subjects see the author's "Procision of Mensurements." 
Thus, calling the last place retained the rth place, the limits of the rejection error entering into that place are +0.5 and -0.5 , and as all amounts between these limits are equally likely to occur, the average rejection error in the long run will be 0.25 in the rth place.

Law and Amount of Accumulated Rejection Error. - Let the last place of significant figures retained in a number, e.g. the fourth, fifth, etc., be called the $r$ th place. Then the error entering from rejection of figures beyond the $r$ th will be at most \pm 5 in the $(r+1)$ place, and any error between these limits, +5 and -5 in the $(r+1)$ place, will be equally likely to occur in any given case, and therefore will be of equal frequency in the long run. The average rejection error in any considerable number of rejections will therefore be \pm 2.5 in the $(r+1)$ place. If then in direct processes of multiplication, division, evolution, or involution (separate or combined) each factor, product, and quotient in the operation be carried out to the same number $r$ of places, what will be the accumulated fractional rejection error if $n$ such rejections are made during the entire operation? This error we shall call for brevity the computation error, or simply the rejection error. This question might easily be answered in a form giving the average accumulated error, but we are at present concerned chiefly with the maximum possible error, since our object is to frame rules which will reduce the worst possible computation error to negligible dimensions. The maximum computation error would arise when every single rejection error was 5 in the $(r+1)$ place, and all had the same sign; and this would be the greatest fraction of the final result when that result and all the factors (and therefore all the intermediate products, quotients, etc.) began with $\mathbf{I}$ and had $\mathrm{o}$ in the other places, i.e. were each $\mathrm{IO}^{r-1}$ with the decimal point wherever it might happen to stand. If there were $n$ of these factors, products, quotients, etc., at which rejections were made, the maximum possible computation error in the result would, therefore, be $n \times 5$ in the $(r+1)$ place, and the fractional error would be $5 n / 10^{r}$. Since this maximum error would be exceedingly rare unless $n$ were very small, and any approaching one-half of it would be very rare, we may properly assume that our rules will be sufficiently stringent if we allow this maximum error to have the same magnitude as the desired accuracy in the result as expressed on the basis of the precision measure, or deviation measure, explained at page xlii. To determine most simply what number $r$ of places this limitation would call for in the processes under consideration, let us take specific cases. Suppose the work is desired to possess an accuracy of $\mathbf{I}$ per cent. Then $5 n / 10^{r}$ must be equal to or less than I per cent; i.e. $5 n=1 / 100$. Hence, we have to solve

$$
\frac{5 n}{10^{r}}=\frac{1}{100}
$$

$$
\begin{aligned}
\text { By inspection, if } & r=3,5 n=10, \therefore n=2, \\
\text { if } & r=4,5 n=100, \therefore n=20 .
\end{aligned}
$$

But obviously $n$ will almost never be as small as 2, and rarely as large as 20 , lying with greatest frequency between 5 and 10 and averaging below 7 . This will give a maximum error of 0.0035 or $\frac{1}{3}$ per cent with $n=7, r=4$, which would be insignificant, and rising to I per cent only when $n=20$. Hence, in work of multiplication, division, etc., where I per cent accuracy, or a little better, is desired (remembering that by this we mean only a deviation measure of $\mathbf{I}$ per cent) $r=4$ will be an entirely safe but not an excessive value; that is, the reten- 
tion of four significant figures throughout will insure entirely sufficient freedom from computation error in every case when the number of rejections is less than the very unusual total of about 20 , and fewer places would not be warranted. Similarly five places will suffice for work to o.I per cent, i.e. better than 1 per cent, but not much better than 0.1 per cent; six places for work of 0.01 per cent, and $80 \mathrm{on}$.

As to relaxation of the rules in special cases, it is evident that but little can be safely done. The maximum error with $n=7, r=4$, will be 0.35 per cent, and an error of $\frac{t}{\xi}$ of this, say, of o. I per cent, will not be of very uncommon occurrence. In fact, with only two rejections the error might be 0.1 per cent. Hence, fuur places cannot be considered as safe to 0.1 per cent, even for short computations. Four places might properly enough be used in short computations up to $\frac{1}{2}$ per cent. Similar statements of course hold for the other rules.

When logarithms are used for multiplication, division, etc., tables should be used giving the mantissa to the same number of places of figures as required by the foreguing rules for direct multiplication and division. Hence, one would employ four-place tables for I per cent work, five-place tables for 0.1 per cent work, and so on. The numbers, antilogs, and mantissæ should all be carried out to the same number of places. This conforms to the customary and only convenient practice.

This rule is based primarily on the fact, next to be shown, that under them the maximum computation error in the use of logarithms arises chietly from the rejection in the numbers and antilogs themselves and not sensibly from the rejected places in the logarithms. In logarithms, a change of the rth figure by $\mathrm{I}$ produces the sane fractional error in the antilog whatever its value, viz. $2.4 / 10^{r}$, as may be seen easily by inspection of tables. Hence, as the maximum rejection error in the tabular value of a logarithm is 5 in the $(r+1)$ place of the mantisse, which may be doubled by the process of interpolating, the maximum fractional error in a result due to the maximum rejection error in any mantissa is $2.4 / 10$. But if only $r$ places are retained in the number or antilog, the maximum error in it due to rejection of its further places is $5 / 10^{r}$, compared to which $2.4 / 10^{\prime}$ is negligible. Hence, as the number of rejections from numher and antilug together is usually about the same as from mantisse, the accumulated error will be due almost wholly to the rejections from the numbers and antilogs. And of these rejections there will ordinarily be about as many if the computation is carried ont by Ingarithms as if by direct multiplication or division. The above rule is thus justified.

In addition or subtraction the maximum rejection error will be obviously $5 n$ in the $(r+1)$ place. Inder the above stated rule that the weakest quantity shall be earried to four significant figures (or two uncertain places) for I per cent work, the smallest value of the deviation measure or uncertainty of the result will be 10 in the rth place, which is 100 in the $(r+1)$ place. Hence,

$$
5 n \geq 100 . \therefore n=20 \text {. }
$$

The maximum accumulated error would then attain the size of the smallest deviation measure, id. the worst possible case would occur, only when the number of rejections was as great as twenty. Hence, the rule of four places for 1 per cent work, five for 0.1 jer cent work, and so un, as before given, is sufficient. 

TABLES 


\section{FOUR PLACE LOGARITHMS.}

LOGS. 4 PL.

\begin{tabular}{|c|c|c|c|c|c|c|c|c|c|c|c|c|c|c|}
\hline No. & 0 & I & 2 & 3 & 4 & 5 & 6 & 7 & 8 & 9 & \multicolumn{4}{|c|}{$\begin{array}{l}\text { INTERPOLATION } \\
\text { TABLES. }\end{array}$} \\
\hline .00 & .0000 & .0004 & .0009 & .0013 & .0017 & .0022 & .0026 & .0030 & .0035 & .0039 & 88 & 36 & 34 & 32 \\
\hline 101 & 0043 & 0048 & 0052 & $\infty_{5} 6$ & 0060 & $\infty 065$ & 0069 & $\infty 073$ & $\infty 0_{77}$ & 0082 & 4 & 4 & 3 & 3 \\
\hline .02 & 0086 & 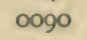 & 0095 & $\infty 099$ & 0103 & 0107 & O111 & 0116 & OI 20 & 0124 & 8 & 7 & 7 & 6 \\
\hline .03 & 0128 & or 33 & 0137 & 0141 & 0145 & 0149 & OI54 & OI $5^{8}$ & oI 62 & oI 66 & II & II & 10 & I0 \\
\hline .04 & 0170 & ol 75 & 0179 & $\mathrm{O} 183$ & 0187 & 191 & 0195 & org9 & 0204 & 0208 & 15 & 14 & 14 & I3 \\
\hline 1.05 & .0212 & .0216 & .0220 & .0224 & .0228 & .0233 & .0237 & $.024 I$ & .0245 & .0249 & 19 & 18 & 17 & 16 \\
\hline .06 & 0253 & 0257 & 0261 & 0265 & 0269 & 0273 & 0278 & 0282 & 0286 & 0290 & 23 & 22 & 20 & 19 \\
\hline .07 & 0294 & 0298 & 0302 & 0306 & 0310 & 0314 & 0318 & 0322 & 0326 & 0330 & 27 & 25 & 24 & 22 \\
\hline .08 & 0334 & $033^{8}$ & 0342 & 0346 & O350 & 0354 & $035^{8}$ & 0362 & 0366 & 0370 & 30 & 29 & 27 & 26 \\
\hline .09 & 0374 & 0378 & $\mathrm{O}_{382}$ & 0386 & 0390 & O394 & 0398 & 0402 & 0406 & 0410 & 34 & 32 & 31 & 29 \\
\hline 1.0 & .0000 & .0043 & .0086 & .0128 & .01 & & .0253 & .0294 & .0334 & .0374 & 30 & 28 & 26 & 24 \\
\hline .1 & 0414 & 0453 & $049^{2}$ & 0531 & 0569 & 0607 & 0645 & 0682 & 0719 & 0755 & 3 & 3 & 3 & 2 \\
\hline .2 & 0792 & 0828 & 0864 & 0899 & 0934 & 0969 & 1004 & 1038 & 1072 & 6 & 6 & 6 & 5 & 5 \\
\hline .3 & II 39 & I 173 & 1206 & 1239 & 1271 & 1303 & 1335 & 1367 & I 399 & 1430 & 9 & 8 & 8 & 7 \\
\hline 4 & 1461 & 1492 & 1523 & 1553 & 1584 & 614 & 1644 & I673 & 1703 & 1732 & 12 & II & 10 & 10 \\
\hline I. 5 & .1761 & .1790 & .1818 & .1847 & .1875 & 1903 & .193I & 1959 & .1987 & .2014 & 15 & 14 & 13 & 12 \\
\hline .6 & $204 I$ & 2068 & 2095 & 2122 & 2148 & 2175 & 2201 & 2227 & 2253 & 2279 & 18 & 17 & 16 & 14 \\
\hline .7 & 2304 & $233^{\circ}$ & 2355 & 2380 & 2405 & 430 & 2455 & 2480 & 2504 & 2529 & 21 & 20 & 18 & 17 \\
\hline .8 & 2553 & 25: & 2601 & 2625 & 2648 & 672 & 2695 & 2718 & 2742 & 2765 & 24 & 22 & $2 I$ & 19 \\
\hline .9 & 8 & 281 & 2833 & 2856 & 2878 & 900 & 2923 & 2945 & 2967 & 2989 & 27 & 25 & 23 & 22 \\
\hline 2.0 & .3010 & $\cdot 3$ & 3 & .30 & .30 & & 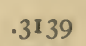 & .3160 & $.318 I$ & & 22 & 20 & 18 & 16 \\
\hline .I & 3222 & & & 3284 & & & 5 & 55 & 3385 & & 2 & 2 & 0 & 2 \\
\hline .2 & 3424 & 34 & 34 & & 35 & & $354^{\mathrm{I}}$ & 50 & & 8 & 4 & 4 & 4 & 3 \\
\hline .3 & 3617 & 36 & 3655 & 3674 & 3692 & I & 3729 & 3747 & 3766 & 3784 & 7 & 6 & 5 & 5 \\
\hline .4 & $3^{802}$ & 3820 & $3^{8} 3$ & 3856 & 387 & 392 & 3909 & 3927 & 3945 & 3962 & 9 & 8 & 7 & 6 \\
\hline 2.5 & .3979 & .3997 & .4014 & $.403 I$ & .40 & 5 & .4082 & .40 & .4116 & & II & 10 & 9 & 8 \\
\hline .6 & 4150 & $4 I$ & 41 & 42 & 4 & 32 & 4249 & 4265 & & & 13 & 12 & II & 10 \\
\hline 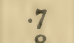 & $43^{14}$ & 4330 & 4346 & 4362 & $437^{8}$ & 393 & 4409 & 4425 & 10 & 4456 & 15 & 14 & 13 & II \\
\hline .8 & 4472 & 4487 & 45 & 45 & 45 & 8 & $45^{64}$ & 45 & 94 & 4609 & 18 & 16 & 14 & 13 \\
\hline 0 & 4624 & 4639 & 4654 & 4669 & 46 & 698 & 4713 & 4728 & 4742 & 4757 & 20 & 18 & 16 & 14 \\
\hline .0 & .477 & & & $.48 \mathrm{I} 4$ & & & & & 86 & & 15 & 14 & 13 & 12 \\
\hline . & 4 & 4928 & 4942 & 4955 & & & 4997 & 5011 & 24 & $5^{\circ} 3^{8}$ & 2 & I & I & I \\
\hline .2 & 5051 & 5065 & 50 & & & & $5^{132}$ & & & $5^{172}$ & 3 & 3 & 3 & 2 \\
\hline .3 & 5185 & 5198 & 5211 & 522 & $5^{2}$ & & $5^{26} 3$ & 5276 & 5289 & & 5 & 4 & 4 & 4 \\
\hline .4 & 5315 & 5328 & 5340 & 5353 & 53 & 8 & 5391 & 5403 & 5416 & 5428 & 6 & 6 & 5 & 5 \\
\hline $3 \cdot 5$ & $.544 \mathrm{I}$ & .5453 & .5465 & $.547^{8}$ & .54 & 2 & .5515 & .55 & .5 & .55 & 8 & 8 & 8 & 6 \\
\hline & $55^{63}$ & & & & & & & & & 5670 & 9 & 8 & 8 & 7 \\
\hline$\cdot 7$ & 5682 & 569 & 57 & 57 & 57 & 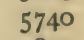 & $575^{2}$ & $57^{6} 3$ & 5775 & 5786 & II & 10 & 9 & 8 \\
\hline & 5798 & 580 & 5821 & $5^{8} 3^{2}$ & & & & & & $5^{899}$ & 12 & II & 10 & 10 \\
\hline .9 & 5911 & 5922 & 5933 & 5944 & 59 & 6 & 5977 & 5988 & 5999 & 6010 & 14 & 13 & 12 & II \\
\hline 4.0 & .6021 & .60 & .60 & .6 & & & & & & & 11 & 10 & . & 8 \\
\hline .I & 6128 & $613^{8}$ & 6149 & & & & & & & & I & I & I & I \\
\hline .2 & 6232 & 6243 & 6253 & 6263 & & & 6294 & & & & 2 & 2 & 2 & 2 \\
\hline .3 & 6335 & 6345 & 6355 & 6365 & 63 & & 6395 & & & & 3 & 3 & 3 & 2 \\
\hline .4 & 6435 & 6444 & 6454 & 6464 & 64 & & 6493 & 6503 & 6513 & 6522 & 4 & 4 & 4 & 3 \\
\hline 4.5 & .6532 & .6542 & .6551 & & & & .65 & & .66 & .6618 & 6 & 5 & 5 & 4 \\
\hline .6 & & 6637 & 6646 & 6656 & & & & & & & 8 & 6 & 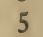 & 5 \\
\hline .7 & 6721 & $673^{\circ}$ & 6739 & 6749 & & 67 & 6776 & & & & 8 & 7 & 6 & 6 \\
\hline .8 & 6812 & 6821 & 6830 & 6839 & 6848 & & 6866 & 6875 & 6884 & 6893 & 9 & 8 & 7 & 6 \\
\hline .9 & 6902 & 6911 & 6920 & 6928 & 6937 & 6946 & 6955 & 6964 & 6972 & $698 \mathrm{I}$ & 10 & 9 & 8 & 7 \\
\hline
\end{tabular}




\section{FOUR PLACE LOGARITHMS.}

\begin{tabular}{|c|c|c|c|c|c|c|c|c|c|c|c|c|c|}
\hline No. & o & I & 2 & 3 & 4 & 5 & 6 & 7 & 8 & 9 & \multicolumn{3}{|c|}{$\begin{array}{l}\text { INTERPOLATION } \\
\text { TABLES. }\end{array}$} \\
\hline 5.0 & .6990 & .6998 & .7007 & .7016 & .7024 & .7033 & .7042 & .7050 & & .7067 & 9 & 8 & 7 \\
\hline .1 & 7076 & $70 S_{4}$ & 7093 & 7101 & 7110 & 7118 & 7126 & 7135 & 7143 & 7152 & I & I & I \\
\hline .2 & 7160 & 7168 & 7177 & 7185 & 7193 & 7202 & 7210 & 7218 & 7226 & 7235 & 2 & 2 & I \\
\hline .3 & $7^{24} 3$ & 7251 & 7259 & 7267 & 7275 & 7284 & 7292 & 7300 & 7308 & 7316 & 3 & 2 & 2 \\
\hline .4 & 7324 & $733^{2}$ & 7340 & 7348 & 7356 & 7364 & 7372 & 7380 & 7388 & 7396 & 4 & 3 & 3 \\
\hline 5.5 & .7404 & .7412 & .7419 & .7427 & .7435 & .7443 & $.745^{1}$ & .7459 & .7466 & .7474 & 5 & 4 & 4 \\
\hline .6 & 7482 & 7490 & 7497 & 7505 & 7513 & 7520 & 7528 & 7536 & 7543 & $755^{1}$ & 5 & 5 & 4 \\
\hline .7 & 7559 & 7566 & 7574 & $75^{82}$ & 7589 & 7597 & 7604 & 7612 & 7619 & 7627 & 6 & 6 & 5 \\
\hline .8 & 7634 & $76+2$ & 7649 & 7657 & 7664 & $767^{2}$ & 7679 & 7686 & 7694 & 7701 & 7 & 6 & 6 \\
\hline .9 & 7709 & 7716 & $77^{23}$ & 7731 & $773^{8}$ & 7745 & $775^{2}$ & 7760 & 7767 & 7774 & 8 & 7 & 6 \\
\hline 6.0 & $.77^{82}$ & $.77^{89}$ & .7796 & $.780_{3}$ & .7810 & .7818 & .7825 & .7832 & $.7^{8} 39$ & .7846 & 7 & & 6 \\
\hline .1 & 7853 & 7860 & 7568 & 7875 & 7882 & 7889 & 7896 & 7903 & 7910 & 7917 & 1 & & I \\
\hline .2 & 7924 & 7931 & $793^{8}$ & 7945 & 7952 & 7959 & 7966 & 7973 & 7980 & 7987 & I & & I \\
\hline .3 & 7993 & 8000 & 8007 & 8014 & 8021 & 8028 & 8035 & $8 \mathrm{O}_{4} \mathrm{I}$ & 8048 & 8055 & 2 & & 2 \\
\hline 4 & 8062 & 8069 & 8075 & 8082 & 8089 & 8096 & 8102 & 8109 & 8116 & 8122 & 3 & & 2 \\
\hline 6.5 & .8129 & .8136 & .8142 & .8149 & .8156 & .8162 & .8169 & .8176 & .8182 & .8189 & 4 & & 3 \\
\hline .6 & 8195 & 8202 & 8209 & 8215 & 8222 & 8228 & 8235 & 8241 & 8248 & 8254 & 4 & & 4 \\
\hline .7 & $\delta_{261}$ & 8267 & 8274 & 8280 & 8287 & 8293 & 8299 & 8306 & 8312 & 8319 & 5 & & 4 \\
\hline .8 & 8325 & $833 i$ & $833^{8}$ & 8344 & 8351 & 8357 & 8363 & 8370 & 8376 & $8_{3} 82$ & 6 & & 5 \\
\hline .9 & 8388 & 8395 & 8401 & 8407 & 8414 & 8420 & 8426 & 8432 & 8439 & 8445 & 6 & & 5 \\
\hline 7.0 & $.845^{1}$ & .8457 & .8463 & .8470 & .8476 & .8482 & .8488 & .8494 & .8500 & .8506 & 6 & & 5 \\
\hline .1 & 8513 & 8519 & 8525 & $8_{53} 1$ & 8537 & $8_{543}$ & 8549 & 8555 & $856 I$ & 8567 & 1 & & I \\
\hline .2 & 8573 & 8579 & $8_{5} 8_{5}$ & 8591 & 8597 & 8603 & 8609 & 8615 & 8621 & 8627 & I & & I \\
\hline .3 & 8633 & 8639 & 8645 & 8651 & 8657 & $866_{3}$ & 8669 & 8675 & $868 I$ & 8686 & 2 & & 2 \\
\hline 4 & 8692 & 8698 & 8704 & 8710 & 8716 & 8722 & 8727 & 8733 & 8739 & 8745 & 2 & & 2 \\
\hline 7.5 & $.875 \mathrm{I}$ & .8756 & .8762 & .8768 & .8774 & .8779 & .8785 & .8791 & .8797 & .8802 & 3 & & 3 \\
\hline .6 & 8508 & 8814 & 8820 & 8825 & $88_{31}$ & 88 & 8842 & 8848 & 88 & $88_{59}$ & 4 & & 3 \\
\hline .7 & 8865 & 8871 & 8876 & 8882 & 8887 & 8893 & 8899 & 8904 & 8910 & 8915 & 4 & & 4 \\
\hline .8 & 8021 & 8927 & 8932 & $893^{8}$ & 8943 & 8949 & 8954 & 8960 & 8965 & 8971 & 5 & & 4 \\
\hline .9 & 8976 & 8982 & 8957 & 8993 & 8998 & 9004 & 9009 & 9015 & 9020 & 9025 & 5 & & 5 \\
\hline 8.0 & .9031 & .9036 & .9042 & .9047 & .9053 & $.905^{8}$ & .9063 & .9069 & .9074 & 9079 & 6 & & 5 \\
\hline . 1 & $90 S_{5}$ & $90 y 0$ & yog6 6 & 9101 & 9106 & 9112 & 9117 & 9122 & 9128 & 9133 & 1 & & I \\
\hline .2 & $913^{8}$ & 9143 & 9149 & 9154 & 9159 & 9165 & 9170 & 9175 & 9180 & 9186 & I & & 1 \\
\hline 3 & 9191 & 9196 & 9201 & 9206 & 9212 & 9217 & 9222 & 9227 & 92.32 & $923^{8}$ & 2 & & 2 \\
\hline 4 & 9243 & 9248 & 9253 & $925^{8}$ & 9263 & 9269 & 9274 & 9279 & 9284 & 9289 & 2 & & 2 \\
\hline 8.5 & .9294 & .9299 & .9304 & .9309 & .9315 & .9320 & .9325 & .9330 & .9335 & .9340 & 3 & & 3 \\
\hline .6 & 9345 & 9350 & 9355 & 9360 & 9365 & 9.370 & 9375 & $93^{50}$ & $93^{85}$ & 93 & 4 & & 3 \\
\hline .7 & 9,395 & 9400 & 9405 & 9410 & 94 & & 9425 & 94,30 & 94 & 9 & 4 & & 4 \\
\hline .8 & 9445 & 9450 & 9455 & 9460 & 9465 & 9469 & 9474 & 9479 & $94 \sqrt{4}$ & 9489 & 5 & & 4 \\
\hline .9 & 9404 & $94 \% 9$ & 9504 & 9509 & 9513 & 9518 & 9523 & 9528 & 9533 & $953^{8}$ & 5 & & 5 \\
\hline 9.0 & .9542 & .9547 & 2 & .9 & .95 & .9566 & 8 & $.957^{6}$ & $.95^{81}$ & .9586 & 5 & & 4 \\
\hline 1 & 9500 & 9595 & $9(x) 0$ & $9^{605}$ & $9(x \times x)$ & 9614 & 9619 & gri24 & $9^{6 / 28}$ & 9633 & I & & 0 \\
\hline .2 & $963^{8}$ & 9643 & 9647 & 9652 & 9657 & $966 \mathrm{i}$ & 9666 & 9671 & $9^{675}$ & 9680 & $i$ & & 1 \\
\hline .3 & $965_{5}$ & 9659 & 9694 & 9699 & 9703 & 9708 & 9713 & 9717 & $97^{22}$ & 9727 & 2 & & 1 \\
\hline .4 & $973^{1}$ & 97,36 & 9741 & 9745 & 9750 & 9754 & 9759 & $97^{6} 3$ & $97^{68}$ & 9773 & 2 & & 2 \\
\hline 9.5 & .9777 & $.97^{\mathrm{S}_{2}}$ & .9786 & .9791 & .9795 & .9800 & .9805 & 9809 & .9814 & 8818 & 3 & & 2 \\
\hline 6 & 9823 & 9827 & $9^{s_{32}}$ & $983^{6}$ & 9841 & 9845 & $98_{50}$ & 9854 & $9^{8} 59$ & 9863 & 3 & & 2 \\
\hline .7 & $9 \$ 68$ & 9872 & 9877 & 9881 & $9 \$ 86$ & 9800 & $9 \sin _{4}$ & 9899 & 9003 & 9008 & 4 & & 3 \\
\hline .8 & 9912 & 9917 & 9921 & 9926 & 9930 & 9034 & 9939 & 9943 & 9048 & 9952 & 4 & & 3 \\
\hline .9 & 9056 & 9961 & 9965 & 9969 & 9074 & $907^{8}$ & $908_{3}$ & 9987 & 999 & 9906 & 5 & & 4 \\
\hline
\end{tabular}


ANTILOGS. $4 \mathrm{PL}$.

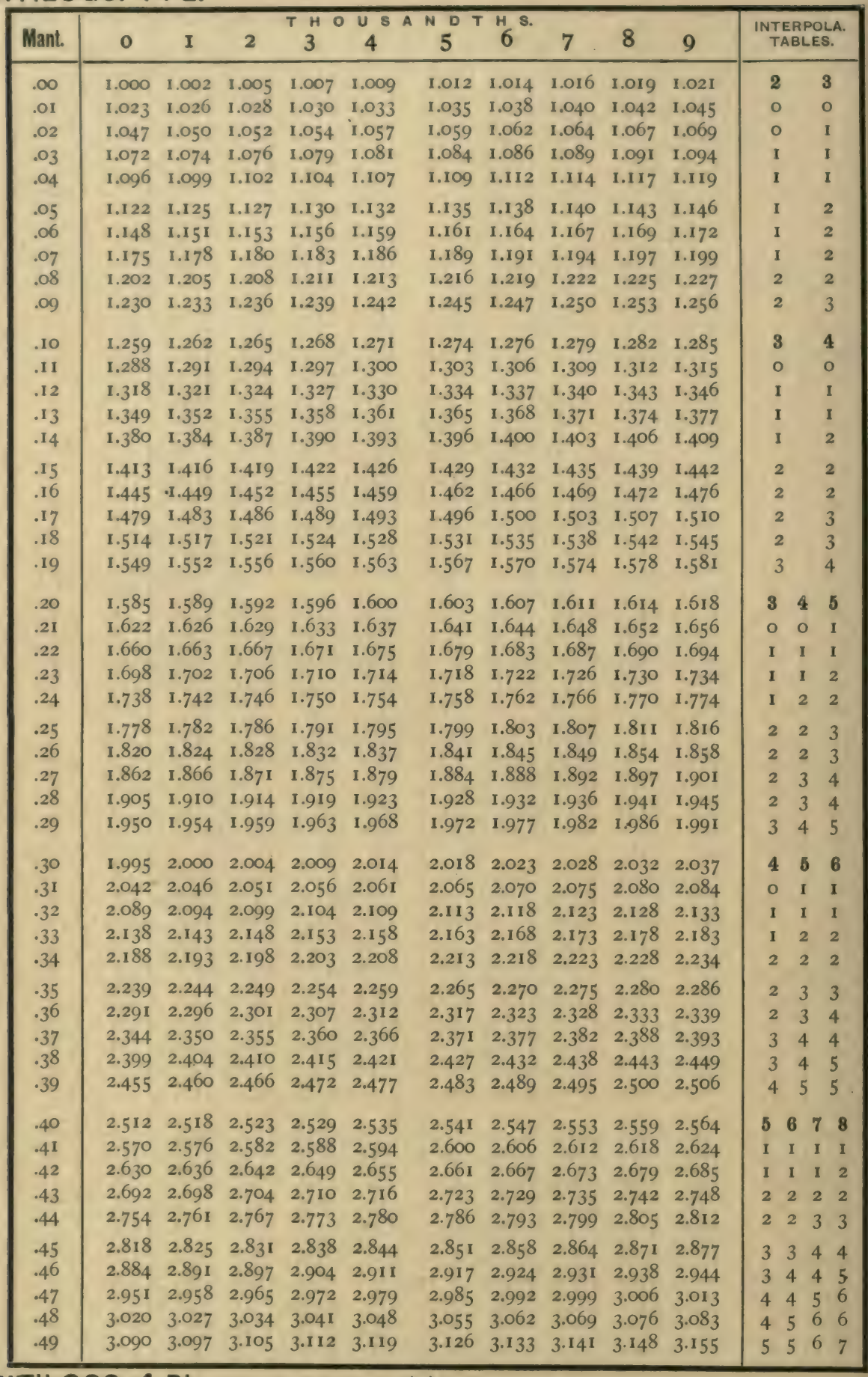




\section{FOUR PLACE ANTILOGARITHMS.}

4 PL. ANTILOGS.

\begin{tabular}{|c|c|c|c|c|c|c|c|c|c|c|c|}
\hline Mant. & 0 & I & 2 & $\begin{array}{c}T H \\
3\end{array}$ & $\begin{array}{c}1 . u^{8} \\
4\end{array}$ & ${ }_{5}^{N}$ & T $\frac{H}{6}$ & 7 & 8 & 9 & $\begin{array}{l}\text { NTERPOLA. } \\
\text { TABLES. }\end{array}$ \\
\hline ;o & 162 & 3.170 & 3.177 & 3.184 & 3.192 & 3.199 & 3.206 & 3.214 & 3.221 & 3.228 & $\begin{array}{lll}7 & 8 & 9\end{array}$ \\
\hline 51 & & 3.243 & & 3.258 & 3.266 & 3.273 & 3.281 & & & & I I I \\
\hline .52 & 311 & 3.319 & $3 \cdot 327$ & $3 \cdot 334$ & $3 \cdot 34^{2}$ & 3.350 & 3.357 & 3.365 & & & 122 \\
\hline .53 & $3 \cdot 358$ & $3 \cdot 396$ & 3.404 & 3.412 & 3.420 & 3.428 & 3.436 & $3 \cdot 443$ & & & $\begin{array}{lll}2 & 2 & 3\end{array}$ \\
\hline .54 & $3-467$ & $3 \cdot 475$ & $3 \cdot 4 \mathrm{~S}_{3}$ & $3-491$ & 3.499 & 3.508 & 3.516 & $3 \cdot 524$ & 2 & 3.5 & $\begin{array}{lll}3 & 3 & 4\end{array}$ \\
\hline .55 & $3 \cdot 54^{8}$ & 3.556 & 3.565 & 3.573 & $3.5^{81}$ & 3.589 & 3.597 & 3.606 & 3. & 3.622 & $\begin{array}{lll}4 & 4 & 5\end{array}$ \\
\hline & & & & 3.656 & 3.664 & 3.673 & 3. 681 & 3.690 & & & 455 \\
\hline .57 & $3.7^{15}$ & 3.724 & 3.733 & 3.741 & 3.750 & $3.75^{8}$ & 3.767 & 3.776 & $3 \cdot 78_{4}$ & & 566 \\
\hline $.5^{8}$ & 3.502 & 3.811 & 3.819 & 3.828 & 3.837 & 3.846 & 3.855 & 3.864 & & & $\begin{array}{lll}6 & 6 & 7\end{array}$ \\
\hline .59 & 3.890 & $3 . \$ 99$ & 3.908 & 3.917 & 3.926 & 3.936 & 3.945 & 3.954 & & 3.972 & 67 \\
\hline .60 & $3.98 \mathrm{I}$ & 3.990 & 3.999 & 4.009 & 4.018 & 4.027 & 4.036 & 4.046 & 5 & 4.064 & 9101118 \\
\hline $.6 \mathrm{t}$ & 4 & 4.0 & 4.0 & 4.102 & 4.111 & 4.121 & 4.130 & 4.140 & & 59 & $\begin{array}{llll}1 & 1 & 1 & 1\end{array}$ \\
\hline .62 & 4 & 4.1 & $4 \cdot 1$ & 4.198 & 4.207 & 4.217 & 4.227 & 4.2 & & & $\begin{array}{llll}2 & 2 & 2 & 2\end{array}$ \\
\hline .63 & 6 & 4.276 & $4.28_{5}$ & 4.295 & 4.305 & $4.3^{15}$ & 4.325 & $4 \cdot 3$ & 4. & & $\begin{array}{llll}3 & 3 & 3 & 4\end{array}$ \\
\hline .64 & 4.305 & $4 \cdot 375$ & $4 \cdot 385$ & 4.395 & 4.406 & $4 \cdot 416$ & $4 \cdot 426$ & 4. & & & 4445 \\
\hline 65 & 4.467 & 4.477 & 4.487 & 4.498 & 4.508 & 4.519 & 4.529 & $4 \cdot 5$ & 0 & & 5566 \\
\hline & 4. & $4 \cdot 5$ & 4. & 4.603 & 4.613 & 24 & 4.634 & & & & $\begin{array}{llll}5 & 6 & 7 & 7\end{array}$ \\
\hline .67 & 4.677 & 4.688 & 4.699 & 4.710 & 4.721 & $4.73^{2}$ & 4.742 & & & & 678 \\
\hline .6 & 4.786 & 4.797 & 4.808 & 4.819 & $4.8_{31}$ & 4.842 & 4.853 & 4.8 & & & 78910 \\
\hline .69 & 4.898 & 4.909 & 4.920 & 4.932 & 4.943 & 4.955 & 4.966 & 4.977 & 4. & 5. & 891011 \\
\hline .70 & & 5.02 & & 5.047 & $5.05^{8}$ & 5.070 & 5.082 & 5.0 & & & 12131415 \\
\hline .71 & 5.129 & 5.140 & 5.152 & 5.164 & 5.176 & 5.188 & 5.200 & 5.212 & & & 1112 \\
\hline .72 & 5.248 & 5.260 & 5.272 & 5.284 & 5.297 & $5 \cdot 309$ & $5 \cdot 321$ & $5 \cdot 333$ & & & $\begin{array}{llll}2 & 3 & 3 & 3\end{array}$ \\
\hline .73 & $5 \cdot 37$ & $5 \cdot 3_{3}$ & 5.395 & 5.408 & 5.420 & 5.433 & 5.445 & & & & 4445 \\
\hline .74 & 5.495 & $5 \cdot 5$ & $5 \cdot 5$ & $5 \cdot 534$ & $5 \cdot 546$ & 5.559 & 5.572 & $5.5^{8} 5$ & $5 \cdot 5$ & $5.6 \mathrm{ro}$ & 5566 \\
\hline & 5.623 & 5.6 & 5.649 & 5.662 & 5.675 & 5.689 & 5.702 & 5. & 8 & & 6778 \\
\hline & 5.75 & 5.76 & 5.781 & 5.794 & 5.808 & 5.821 & 5.834 & 5.848 & & & 7889 \\
\hline .7 & 5.858 & 5.90 & 5.916 & 5.929 & 5.943 & 5.957 & 5.970 & 5.984 & & 2 & 891011 \\
\hline 8 & 026 & 6.039 & 6.053 & 6.067 & 6.081 & 5 & 6.109 & 6.124 & 6. & 2 & 10101112 \\
\hline .79 & 6.166 & 6.180 & 6.194 & 6.209 & 6.223 & 6.237 & 6.252 & 6.266 & 6 & 6. & 11121314 \\
\hline & & & & 6.353 & & 33 & 6.397 & & & & 16171819 \\
\hline .81 & $\epsilon$ & ( & & 6.501 & 6.516 & 6.531 & 6.546 & 6. & & & 2222 \\
\hline .8 & 07 & 6.6 & 6. & 6.653 & 6.668 & 6.683 & 6.699 & 6. & & & 3344 \\
\hline .8 & 61 & 6.776 & 6.7 & 6. 508 & 6.823 & 6.839 & 6.855 & 6.871 & & & $\begin{array}{llll}5 & 5 & 5 & 6\end{array}$ \\
\hline .84 & 18 & 6.934 & & 6.966 & 6.982 & 6.998 & 7.015 & $7 \cdot c$ & 7. & $7 \cdot 6$ & 6778 \\
\hline .85 & 9 & 096 & & 7.129 & 7 & 61 & 7.178 & & & & 89910 \\
\hline & 44 & 7.261 & 7.278 & 7.295 & 7.311 & 8 & $7 \cdot 345$ & 7. & & & 10101111 \\
\hline 87 & 13 & 7.430 & 7.4 & $7 \cdot 464$ & $7 \cdot 482$ & & $7 \cdot 516$ & & & & 11121313 \\
\hline .88 & 86 & $7 . \mathrm{fO}_{3}$ & 7.6 & 7.638 & 7.656 & & 7.691 & & & & 13141415 \\
\hline .89 & $7 \cdot 762$ & $7 \cdot 780$ & 7.798 & 7.816 & 7.834 & 7.852 & 7.870 & 7.589 & 7.907 & $7 \cdot 925$ & 14151617 \\
\hline & & 7.962 & & 7 & & & & & & & 20212228 \\
\hline & & 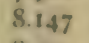 & 8.166 & 8.185 & 8.2 & & 8. & 8.2 & & & 2222 \\
\hline & 8 & $\mathrm{~s}$ & 8. & 8.375 & 8.3 & & & & & & 4445 \\
\hline & & 3.5 & 8. 5 & 8.570 & 8.5 & & & & & & 6677 \\
\hline .94 & 8.710 & 8.730 & 8.750 & 8.770 & 8.790 & 0 & $8.8_{31}$ & $8.8_{51}$ & $8.87^{2}$ & 8.892 & 8899 \\
\hline & 8. & 8.933 & & 8.974 & & & & & & 9.0099 & 10111112 \\
\hline & 9.120 & 9.141 & 9.162 & $9.1 s_{3}$ & 9.204 & 9.226 & 9.247 & $9.26 \mathrm{~s}$ & 9.290 & $9 \cdot 311$ & 12131314 \\
\hline$?$ & 9.3 .33 & 9.354 & $9 \cdot 37^{6}$ & 9.397 & & & $9 \cdot 4^{1 / 2}$ & 9.48 .4 & 9.506 & $9 \cdot 528$ & 14151516 \\
\hline .98 & 9.550 & 9.572 & 9.594 & 9.616 & 9.638 & 9.66t & $9.6 S_{3}$ & 9.705 & 9.727 & 9.750 & 16171818 \\
\hline .99 & 9.772 & $9 \cdot 775$ & 9.817 & 9.840 & 9.863 & 9.856 & 9.908 & 0.931 & 9.954 & 9.977 & 18192020 \\
\hline
\end{tabular}




\begin{tabular}{|c|c|c|c|c|c|c|c|c|c|c|c|}
\hline No. & 0 & I & 2 & 3 & 4 & 5 & 6 & 7 & 8 & 9 & $\begin{array}{c}\text { INTERPOLATION } \\
\text { TABLES. }\end{array}$ \\
\hline 00 & \multicolumn{5}{|c|}{$\overline{\mathbf{I}} .9996 \overline{\mathrm{I}} .9991 \overline{\mathrm{r}} .9987 \overline{\mathrm{I}} .9983$} & \multicolumn{5}{|c|}{ 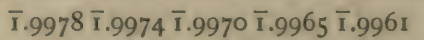 } & \multirow{10}{*}{$\begin{array}{l}\text { Use the frrst ten } \\
\text { lines to avoid in- } \\
\text { terpolating } \\
\text { from } 1.000 \\
\text { to } 1.100 .\end{array}$} \\
\hline & $\overline{\mathbf{I}} .9957$ & 9953 & $994^{8}$ & 9944 & $994^{\circ}$ & 9935 & 9931 & 9927 & 9923 & 9918 & \\
\hline 2 & 9914 & 9910 & 905 & & & 9893 & 9887 & 9884 & 9880 & 9876 & \\
\hline .03 & 9872 & 9867 & 9863 & 9859 & 9855 & 9851 & 9846 & 9842 & 9838 & 9834 & \\
\hline .04 & 9830 & 9825 & 9821 & 9817 & 9813 & 9809 & 9805 & $980 \mathrm{I}$ & 9796 & 9792 & \\
\hline 1.05 & $\overline{\mathrm{I}} .9788 \mathrm{i}$ & $\overline{\mathrm{I}} .9784 \overline{\mathrm{I}}$ & $.9780 \mathrm{I}$ & $.9776 \bar{I}$ & $\overline{1} .9772$ & $\overline{\mathbf{I}} .9767 \bar{I}$ & $\overline{1} .9763 \bar{I}$ & $\bar{I} .9759 \bar{i}$ & $.9755^{\mathrm{I}}$ & $.975^{1}$ & \\
\hline .06 & 9747 & 9743 & 9739 & 9735 & $973^{1}$ & 9727 & 9722 & 9718 & 9714 & 9710 & \\
\hline .07 & & & & & & 9686 & 9682 & 9678 & 9674 & 9670 & \\
\hline .08 & 9666 & 9662 & 9658 & 9654 & 9650 & 9646 & 9642 & $963^{8}$ & 634 & 9630 & \\
\hline .09 & 9626 & 9622 & 9618 & 9614 & 9610 & 9606 & 9602 & 9598 & 9594 & 9590 & \\
\hline 1.0 & -1.00 & 1.9958 & (2) & , & 1.90 & $\overline{\mathbf{I}} .978$ & 1.9 & 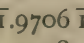 & . & & $-44-40-36-32$ \\
\hline .1 & $\overline{\mathrm{I}} .95^{86}$ & 9547 & 9508 & 9469 & (2) & 9393 & (18 & 8 & I & 45 & $\begin{array}{llll}4 & 4 & 4 & 3\end{array}$ \\
\hline .2 & 9208 & 9172 & & & & & & & 8 & 8894 & 987 \\
\hline$\cdot 3$ & 8861 & 8827 & 87 & $8 / 01$ & 872 & 86 & 8665 & 8633 & 8601 & 0 & 1312 II 10 \\
\hline .4 & 8539 & 8508 & 8477 & 7 & & & 356 & 8327 & 82 & 8 & $\begin{array}{llll}18 & 16 & 14 & 13\end{array}$ \\
\hline 1.5 & $\overline{\mathrm{I}} .8239 \overline{\mathrm{I}}$ & $\overline{1} .8210$ & .818 & 815 & I. 8 & $\overline{\mathrm{I}} .8097 \overline{\mathrm{I}}$ & $8069 \bar{I}$ & I.804 I & I. & 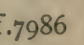 & $\begin{array}{llll}22 & 20 & 18 & 16\end{array}$ \\
\hline .6 & 7959 & 7932 & 790 & 7878 & 7852 & 7825 & 7799 & & 7 & 7721 & $\begin{array}{llll}26 & 24 & 22 & 19\end{array}$ \\
\hline .7 & & & & & & & & & & & $\begin{array}{llll}31 & 28 & 25 & 22\end{array}$ \\
\hline .8 & 7447 & 7 & 7399 & 7 & & & & & & 5 & $\begin{array}{llll}35 & 32 & 29 & 26\end{array}$ \\
\hline 9 & 7212 & 7 & 7 & 7144 & 7122 & 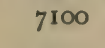 & 7 & 5 & 3 & 7011 & $\begin{array}{llll}40 & 3^{6} & 3^{2} & 29\end{array}$ \\
\hline 2.0 & $\overline{\mathrm{I}} .6990 \overline{\mathrm{i}}$ & I.6968 & 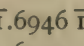 & 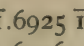 & & $\overline{\mathbf{1}} .688$ & & & & & $-28-26-24-22$ \\
\hline .1 & 6778 & 6757 & 673 & 671 & & 667 & 6655 & & 66 & 6596 & $\begin{array}{llll}3 & 3 & 2 & 2\end{array}$ \\
\hline .2 & 6576 & 6556 & 6536 & 651 & 64 & 6478 & 6 & & & & $\begin{array}{llll}6 & 5 & 5 & 4\end{array}$ \\
\hline .3 & $6_{3} 8_{3}$ & 6364 & 6345 & 63 & 63 & 6289 & 6271 & & & 62 & $\begin{array}{llll}8 & 8 & 7 & 7\end{array}$ \\
\hline .4 & 608 & 6.8 & & & & & & & & 60 & II I0 I0 9 \\
\hline 2.5 & $\overline{\mathrm{I}} .602 \mathrm{I} \overline{\mathrm{I}}$ & $\overline{1} .6003 \overline{\mathbf{I}}$ & .598 & .59 & 2 & $\overline{\mathbf{I}} \cdot 593$ & $3 \bar{I}$ & $\overline{1}$ & $\bar{I}$ & 867 & $141312 \quad 11$ \\
\hline .6 & $5^{850}$ & 5834 & 581 & 5800 & 5784 & 5768 & & & & & $\begin{array}{llll}17 & 16 & 14 & 13\end{array}$ \\
\hline .7 & 5686 & 5670 & & & & & & & & & $\begin{array}{llll}20 & 18 & 17 & 15\end{array}$ \\
\hline .8 & 5528 & 55 & 3 & 5482 & & & & & & & $\begin{array}{llll}22 & 21 & 19 & 18\end{array}$ \\
\hline .9 & 5376 & 5361 & 340 & 53 & 5 & 5302 & 287 & $5^{272}$ & 258 & 5243 & $\begin{array}{llll}25 & 23 & 22 & 20\end{array}$ \\
\hline 3.0 & & I. 5 & & 1 & $\bar{I}$ & & & & & & $18-16-14-12$ \\
\hline .1 & 5086 & 5072 & $505^{8}$ & & & & & & & & $\begin{array}{llll}2 & 2 & \text { I } & \text { I }\end{array}$ \\
\hline .2 & 4948 & 49 & 49 & 4 & 5 & & 0 & & & 8 & $\begin{array}{lll}4 & 3 & 3\end{array}$ \\
\hline$\cdot 3$ & 4815 & 4802 & 4789 & 4776 & 4763 & 0 & & & & & $\begin{array}{lll}5 & 5 & 4\end{array}$ \\
\hline .4 & 4685 & 4672 & & 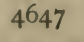 & 4634 & 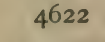 & 4009 & 4597 & $45^{84}$ & 4572 & $\begin{array}{llll}7 & 6 & 6 & 5\end{array}$ \\
\hline 3.5 & $\overline{\mathrm{I}} .4559 \overline{\mathrm{I}}$ & I. 454 & .45 & $\bar{I}$ & .45 & $\overline{\mathrm{I}} .449$ & $.4486 \bar{I}$ & $\bar{I}$ & - & & $\begin{array}{llll}9 & 8 & 7 & 6\end{array}$ \\
\hline .6 & 4437 & 4425 & 44 & & 4389 & & 4365 & 3 & & & II 10 8 \\
\hline .7 & 8 & 43 & & 3 & & & $4^{8}$ & & & 4 & I3 II IO 8 \\
\hline .8 & & & & & 4 & & & & & $4 \mathrm{IOI}$ & 14131110 \\
\hline .9 & & 4078 & & & & & 23 & & & 3990 & $\begin{array}{llll}16 & 14 & 13 & \text { II }\end{array}$ \\
\hline 4.0 & $\overline{\mathrm{I}} .3979 \overline{\mathrm{I}}$ & 1.3909 & & 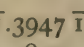 & I. 3936 & $\overline{\mathrm{I}} \cdot 392$ & $.39+5=$ & & 年 & 3883 & $-11-10-9-8$ \\
\hline .1 & 3872 & 3862 & 3851 & & 3830 & 382 & 3809 & & 3788 & 3778 & $\begin{array}{llll}\text { I } & \text { I } & \text { I } & \text { I }\end{array}$ \\
\hline .2 & 3768 & 3757 & 374 & & & & & & & & $\begin{array}{llll}2 & 2 & 2 & 2\end{array}$ \\
\hline$\cdot 3$ & 3665 & & & & & & & & & & $\begin{array}{llll}3 & 3 & 3 & 2\end{array}$ \\
\hline .4 & 3565 & $355^{6}$ & 3546 & 353 & $35^{26}$ & & 3507 & 3497 & 3407 & 3478 & $\begin{array}{llll}4 & 4 & 4 & 3\end{array}$ \\
\hline 4.5 & $\overline{\mathrm{I}} \cdot 3468 \overline{\mathrm{I}}$ & $\overline{\mathrm{I}} \cdot 345^{8 \overline{\mathrm{I}}}$ & $3449 \overline{\mathrm{x}}$ & .343 & I. 3429 & $\overline{\mathrm{I}} \cdot 3420 \overline{\mathrm{I}}$ & $3410 \overline{1}$ & I. $3401 \overline{1}$ & I.3391 I & 3382 & $\begin{array}{lll}6 & 5 & 5\end{array}$ \\
\hline .6 & 3372 & 3363 & 3354 & 3344 & 2325 & & & & 3298 & & 765 \\
\hline .7 & & & & & & & & & & & 8766 \\
\hline .8 & & & & 3161 & & & 3134 & & & & 9876 \\
\hline .9 & 3098 & 3089 & 3080 & 3071 & 3063 & 3054 & 3045 & 3036 & 3028 & 3019 & $\begin{array}{llll}10 & 9 & 8 & 7\end{array}$ \\
\hline
\end{tabular}


4 PL. COLOGS.

\begin{tabular}{|c|c|c|c|c|c|c|c|c|c|c|c|c|c|}
\hline No. & 0 & I & 2 & 3 & 4 & 5 & 6 & 7 & 8 & 9 & \multicolumn{3}{|c|}{$\begin{array}{l}\text { INTERPOLATION } \\
\text { TABLES. }\end{array}$} \\
\hline 5.0 & \multicolumn{5}{|c|}{ 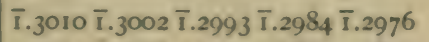 } & \multicolumn{5}{|c|}{ 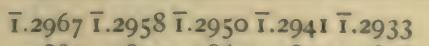 } & $-9-8$ & $-8-$ & -7 \\
\hline .I & 2924 & 2916 & 2907 & 2899 & 2890 & 2882 & 2874 & 2865 & 2857 & 2848 & 11 & 1 & I \\
\hline .2 & 2840 & 2832 & 2823 & 2815 & 2807 & 2798 & 2790 & 2782 & 2774 & $276_{5}$ & 22 & 2 & I \\
\hline$\cdot 3$ & 2757 & 2749 & 2741 & 2733 & 2725 & 2716 & 2708 & 2700 & 2692 & 2684 & 32 & 2 & 2 \\
\hline 4 & 2676 & 2668 & 2660 & 2652 & 2644 & 2636 & 2628 & 2620 & 2612 & 2604 & 43 & 3 & 3 \\
\hline 5.5 & \multicolumn{5}{|c|}{ 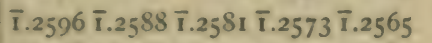 } & \multicolumn{5}{|c|}{ 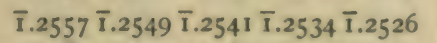 } & 5 & 4 & 4 \\
\hline .6 & 2518 & 2510 & 2503 & 2495 & 2487 & 2480 & $247^{2}$ & 2464 & 2457 & 2449 & 5 & 5 & 4 \\
\hline .7 & 2441 & 2434 & 2426 & 2418 & 2411 & 2403 & 2396 & 2388 & 2381 & 2373 & 6 & 6 & 5 \\
\hline .8 & 2366 & $235^{8}$ & 2351 & 2343 & 2336 & 2328 & 2321 & 2314 & 2306 & 2299 & 7 & 6 & 6 \\
\hline .9 & 2291 & 2284 & 2277 & 2269 & 2262 & 2255 & 2248 & 2240 & 2233 & 2226 & 8 & 7 & 6 \\
\hline 6.0 & \multicolumn{5}{|c|}{$\overline{\mathrm{I}} .2218 \overline{\mathrm{I}} .2211 \overline{\mathrm{I}} .2204 \overline{\mathrm{I}} .2197 \overline{\mathrm{I}} .2190$} & \multicolumn{5}{|c|}{$\overline{\mathrm{I}} .2182 \overline{\mathrm{I}} .2175 \overline{\mathrm{I}} .2168 \overline{\mathrm{I}} .216 \mathrm{I}$ '̄.2154 } & -7 & & -6 \\
\hline I & 2147 & 2140 & 2132 & 2125 & 2118 & 2111 & 2104 & 2097 & 2090 & 2083 & I & & I \\
\hline .2 & 2076 & 2069 & 2062 & 2055 & 2048 & 2041 & 2034 & 2027 & 2020 & 2013 & I & & 1 \\
\hline$\cdot 3$ & 2007 & 2000 & 1993 & 1986 & 1979 & 1972 & 1965 & 1959 & $195^{2}$ & 1945 & 2 & & 2 \\
\hline 4 & $193^{8}$ & 1931 & 1925 & 1918 & 1911 & 1904 & 1898 & 1891 & 1884 & 1878 & 3 & & 2 \\
\hline 6.5 & \multicolumn{5}{|c|}{ 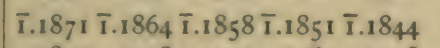 } & \multicolumn{5}{|c|}{ 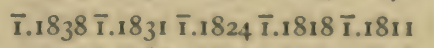 } & 4 & & 3 \\
\hline .6 & 1805 & 1798 & 1791 & 1785 & $177^{8}$ & 1772 & 1765 & 1759 & 1752 & 1746 & 4 & & 4 \\
\hline .7 & 1739 & 1733 & 1726 & 1720 & 1713 & 1707 & 1701 & 1694 & 1688 & $168 \mathrm{I}$ & 5 & & 4 \\
\hline .8 & 1675 & 1669 & 1662 & 1656 & 1649 & 1643 & 1637 & 1630 & 1624 & 1618 & 6 & & 5 \\
\hline .9 & 1612 & 1605 & 1599 & 1593 & 1586 & 1580 & 1574 & 1568 & 1561 & 1555 & 6 & & 5 \\
\hline 7.0 & \multicolumn{5}{|c|}{ 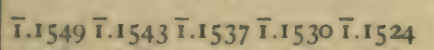 } & \multicolumn{5}{|c|}{ 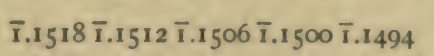 } & -6 & & -5 \\
\hline .1 & 1487 & 1481 & 1475 & 1469 & 1463 & 1457 & 1451 & 1445 & 1439 & 1433 & I & & 1 \\
\hline .2 & 1427 & 1421 & 1415 & 1409 & 1403 & 1397 & I 391 & ${ }_{1} 3_{5}$ & 1379 & 1373 & I & & I \\
\hline$\cdot 3$ & 1367 & 1361 & 1355 & 1349 & 1343 & 1337 & I33I & 1325 & 1319 & 1314 & 2 & & 2 \\
\hline 4 & 1308 & 1302 & 1296 & 1290 & 1284 & 1278 & 1273 & 1267 & 1261 & 1255 & 2 & & 2 \\
\hline 7.5 & \multicolumn{5}{|c|}{ 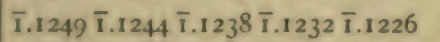 } & \multicolumn{5}{|c|}{ 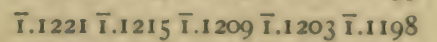 } & 3 & & 3 \\
\hline .6 & 1192 & 1186 & 1180 & 1175 & 1169 & 1163 & $115^{8}$ & 1152 & 1146 & 1141 & 4 & & 3 \\
\hline .7 & 1135 & 1129 & 1124 & 1118 & 1113 & 1107 & IIOI & 1096 & 1090 & 1085 & 4 & & 4 \\
\hline .8 & 1079 & 1073 & 1068 & 1062 & 1057 & $\operatorname{Iog} 1$ & 1046 & 1040 & 1035 & 1029 & 5 & & 4 \\
\hline .9 & 1024 & 1018 & 1013 & 1007 & 1002 & 0996 & 0991 & 0985 & 0980 & 0975 & 5 & & 5 \\
\hline 8.0 & \multicolumn{5}{|c|}{ 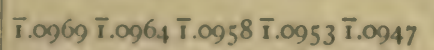 } & \multicolumn{5}{|c|}{ 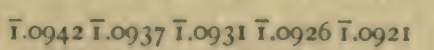 } & -6 & & -6 \\
\hline .1 & o, 15 & 0910 & ono4 & 0899 & 0894 & 0888 & $088_{3}$ & 0878 & 0872 & 0867 & 1 & & 1 \\
\hline .2 & 0862 & $0 \$ 57$ & $\operatorname{os}_{51}$ & 0846 & $084 \mathrm{I}$ & 0835 & 0830 & 0825 & 0820 & 0814 & 1 & & 1 \\
\hline$\cdot 3$ & osog & 0804 & 0799 & 0794 & 0788 & 0783 & 0778 & 0773 & 0768 & 0762 & 2 & & 2 \\
\hline .4 & 0757 & $075^{2}$ & 0747 & 0742 & 0737 & 0731 & 0726 & 0721 & 0716 & O711 & 2 & & 2 \\
\hline 8.5 & \multicolumn{5}{|c|}{ 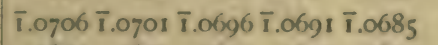 } & \multirow{2}{*}{\multicolumn{5}{|c|}{$\begin{array}{ccccc}\overline{\mathbf{T}} .0680 & \overline{\mathrm{I}} .0675 & \overline{\mathrm{I}} .0670 & \overline{\mathrm{I}} .0665 & \overline{\mathrm{I}} .0660 \\
0630 & 0625 & 0620 & 0615 & 0610\end{array}$}} & 3 & & 3 \\
\hline .6 & 0655 & 0650 & 0645 & 0640 & 0635 & & & & & & 4 & & 3 \\
\hline .7 & obos & of $\times 00$ & 0595 & 0590 & $08^{8} 5$ & 0580 & 0575 & 0570 & 0565 & 0560 & 4 & & 4 \\
\hline .8 & 0555 & 0550 & 0545 & 0540 & 0535 & 0531 & 0526 & 0521 & 0516 & ogit & 5 & & 4 \\
\hline 9 & 0506 & 0501 & 0496 & 0491 & 0487 & 0482 & 0.477 & 0472 & 0467 & 0462 & 5 & & 5 \\
\hline 9.0 & i..0458 & $.0453 \bar{i}$. & $.0448 \pi$ & $.0443 \overline{\mathrm{I}}$ & $.043^{8}$ & $\overline{1} .0434 \overline{\mathrm{I}}$ & $.0429 \overline{\mathrm{I}}$ & $.0424 \overline{1}$ & .0419 T. & .0414 & -6 & & -4 \\
\hline .1 & 0410 & 0405 & 0400 & 0395 & 0398 & 0356 & $03^{81}$ & $037^{6}$ & $037^{2}$ & $0_{3} 67$ & 1 & & o \\
\hline .2 & $\mathrm{O}_{3}(6) 2$ & 0.357 & 0.353 & 0.348 & 0343 & 0339 & 0334 & 0329 & 0325 & 0320 & 1 & & 1 \\
\hline .3 & 0315 & $\mathrm{O}_{311}$ & 0306 & ojor & 0297 & 0292 & 0287 & $028_{3}$ & 0278 & 027.3 & 2 & & 1 \\
\hline 4 & 0269 & 0264 & 0259 & 0255 & 0250 & 0246 & $024 i$ & 0237 & 0232 & 0227 & 2 & & 2 \\
\hline 9.5 & $\overline{1} .0223 \overline{\mathrm{r}}$. & .02187. & $.0214 \bar{T}$ & 1.02097 & .0205 & $\overline{\mathrm{T}} .0200 \overline{\mathrm{I}}$ & .0195 I & i.orgi & i.0186T. & .0182 & 3 & & 2 \\
\hline .6 & 0177 & 0173 & oros & 0164 & 0159 & ol5s & or5o & 0146 & 0141 & 0137 & 3 & & 2 \\
\hline .7 & $\mathrm{OI}_{32}$ & 0128 & 0123 & or19) & 0114 & 0110 & 0106 & olot & 0097 & $\infty 9_{2} 2$ & 4 & & 3 \\
\hline .8 & coss & $\cos _{3}$ & 0079 & $\mathrm{x}^{-14}$ & 0070 & 0066 & 0061 & 0057 & $\cos ^{2}$ & 0048 & 4 & & 3 \\
\hline .9 & on 44 & 0039 & 0035 & $\mathrm{OO}_{31}$ & 0026 & 0022 & 0017 & 0013 & 00019 & 0004 & 5 & & 4 \\
\hline
\end{tabular}





\section{TABLE OF FIVE PLACE LOGARITHMS,}

CONTAINING

An Abbreviated Table for One and Two Place Numbers;

A Table for Five Place Numbers from 1.0 to 1.1 Avoiding Interpolation;

A Table for All Four Place Numbers with Interpolation Tables for the Fifth Place.

\begin{tabular}{|c|c|c|c|c|c|c|c|c|c|}
\hline No. & $\log$. & No. & $\log$. & No. & $\log$. & No. & $\log$. & No. & $\log$. \\
\hline 0 & $-\infty$ & 2.0 & .30103 & 4.0 & .60206 & 6.0 & .77815 & 8.0 & .90309 \\
\hline I & .00000 & 2.1 & .32222 & 4.1 & .61278 & 6.1 & $.7^{8} 533$ & 8.1 & .90849 \\
\hline 2 & .30103 & 2.2 & .34242 & 4.2 & .62325 & 6.2 & .79239 & 8.2 & .91381 \\
\hline 3 & 47712 & 2.3 & .36173 & $4 \cdot 3$ & .63347 & 6.3 & .79934 & 8.3 & 91908 \\
\hline 4 & .60206 & 2.4 & .38021 & $4 \cdot 4$ & .64345 & 6.4 & .80618 & 84 & .92428 \\
\hline 5 & .69897 & 2.5 & .39794 & 4.5 & .65321 & 6.5 & .81291 & 8.5 & .92942 \\
\hline 6 & .77815 & 2.6 & .41497 & 4.6 & .66276 & 6.6 & .81954 & 8.6 & .93450 \\
\hline 7 & .84510 & 2.7 & .43136 & $4 \cdot 7$ & .67210 & 6.7 & .82607 & 8.7 & .93952 \\
\hline 8 & .90309 & 2.8 & .44716 & 4.8 & .68124 & 6.8 & .83251 & 8.8 & $.9444^{8}$ \\
\hline 9 & .95424 & 2.9 & .46240 & 4.9 & .69020 & 6.9 & $.8388_{5}$ & 8.9 & .94939 \\
\hline 1.0 &.$\infty \infty 00$ & 3.0 & .47712 & 5.0 & .69897 & 7.0 & .84510 & 9.0 & .95424 \\
\hline 1.1 & .04139 & 3.1 & .49136 & 5.1 & .70757 & 7.1 & .85126 & 9.1 & .95904 \\
\hline 1.2 & .07918 & 3.2 & .50515 & 5.2 & .71600 & 7.2 & .85733 & 9.2 & .96379 \\
\hline 1.3 & .11 394 & $3 \cdot 3$ & .51851 & $5 \cdot 3$ & .72428 & $7 \cdot 3$ & .86332 & $9 \cdot 3$ & .96848 \\
\hline 1.4 & .14613 & $3 \cdot 4$ & $.5314^{8}$ & 5.4 & .73239 & 7.4 & .86923 & 9.4 & .97313 \\
\hline 1.5 & .17609 & 3.5 & .54407 & 5.5 & .74036 & 7.5 & .87506 & 9.5 & .97772 \\
\hline 1.6 & .20412 & 3.6 & .55630 & 5.6 & .74819 & 7.6 & .8808 ı & 9.6 & .98227 \\
\hline 1.7 & .23045 & 3.7 & .56820 & 5.7 & .75587 & 7.7 & .88649 & 9.7 & .98677 \\
\hline 1.8 & .25527 & 3.8 & $.5797^{8}$ & 5.8 & .76343 & 7.8 & .89209 & 9.8 & .99123 \\
\hline 1.9 & .27875 & 3.9 & .59106 & 5.9 & .77085 & 7.9 & .89763 & 9.9 & .99564 \\
\hline
\end{tabular}




\begin{tabular}{|c|cccccccccc|}
\hline No. & 0 & I & 2 & 3 & 4 & 5 & 6 & 7 & 8 & 9 \\
\hline 1.000 & .00000 & .00004 & .00009 & .00013 & .00017 & .00022 & .00026 & .00030 & .000035 & .00039
\end{tabular}

\begin{tabular}{rr|rrrrrr}
.04 & .001 & 00043 & 00048 & 00052 & 00056 & 00061
\end{tabular}

$\begin{array}{lllllll}.002 & 00087 & 00091 & 00095 & 00100 & 00104\end{array}$

$\begin{array}{lllllllll}.003 & 00130 & 00134 & 00139 & 00143 & 00147\end{array}$

$\begin{array}{llllllll}.04 & 0017300178 & 00182 & 00186 & 00191\end{array}$

$\begin{array}{llllllll}1.005 & .00217 & .00221 & .00225 & .00230 & .00234\end{array}$

$\begin{array}{llllllll}.006 & 00260 & 00264 & 00268 \quad 00273 \quad 00277\end{array}$

$\begin{array}{llllllllll}.007 & 00303 & 00307 & 00312 & 00316 & 00320\end{array}$

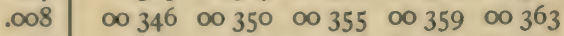

$\begin{array}{lllllllll}.009 & 00389 & 00 & 393 & 00 & 398 & 00402 & 00406\end{array}$

$\begin{array}{lllllll}1.010 & .00432 & .00436 .00441 & .00445 & .00449\end{array}$

$\begin{array}{lllllllll}.011 & 00475 & 0 & 479 & 0 & 484 & 00488 & 00492\end{array}$

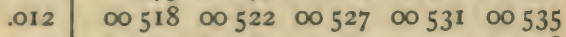

$\begin{array}{lllllllll}.013 & 0 & 561 & 00 & 565 & 0 & 570 & 00574 & 00578\end{array}$

$\begin{array}{llllllll}.014 & 00604 & 00608 & 00612 & 00617 & 00621\end{array}$

$\begin{array}{llllllll}1.015 & .00647 & .00651 & .00655 & .00659 & .00664\end{array}$

$\begin{array}{llllllll}.016 & 00689 & 00694 & 00698 & 00702 & 00706\end{array}$

$\begin{array}{llllllll}.017 & 00732 & 00736 & 00741 & 00745 & 00749\end{array}$

$\begin{array}{llllllllll}.018 & 00775 & 00779 & 00783 & 00788 & 00792\end{array}$

$\begin{array}{lllllll}.019 & 00817 & 00822 & 00826 & 00830 & 00834\end{array}$

$\begin{array}{llllllll}1.020 & .00860 .00864 & .00869 & .00873 & .00877\end{array}$

$\begin{array}{llllllll}.021 & 00903 & 00907 & 00911 & 00915 & 00920\end{array}$

$\begin{array}{llllllll}.022 & 00945 & 00949 & 00954 & 0095^{8} & 00962\end{array}$

$\begin{array}{lllllll}.023 & 00988 & 00992 & 00996 & \text { or } 000 & \text { or } 005\end{array}$

$\begin{array}{llllllll}.024 & \text { or O30 } & \text { or } 034 & \text { OI O38 } & \text { or } 043 & \text { or } 047\end{array}$

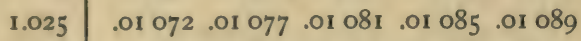

.026 or 115 or 119 OI 123 or 127 or 132

.027 or 157 or 161 or 166 or 170 or 174

.028 or 199 or 204 or 208 or 212 or 216

.029 or 242 or 246 or 250 or 254 ó $25^{8}$

1.030 .OI 284 .OI 288 .OI 292 .OI 296 .Or 301

.031 or 326 or 330 or 334 or 338 or 343

.032 or 368 or 372 or 376 or 38 I or 385

.033 or 410 or 414 or 418 or 423 or 427

.034 or $45^{2}$ or 456 or 460 or 465 or 469

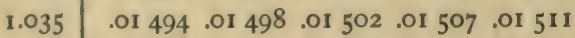

.036 or 536 or 540 or 544 or 549 or 553

.037 or 578 or 582 or $5^{86}$ or 590 or 595

$\begin{array}{lllllll}.038 & \text { or } 620 & \text { or } 624 & \text { or } 628 & \text { or } 632 & \text { or } 636\end{array}$

.039 or 662 or 666 or 670 or 674 or 678

1.040 .or 703 .or 708 .01 712 .OI 716 .or 720

.041 or 745 or 749 or 753 or $75^{8}$ or 762

.042 or 787 or 791 or 795 or 799 or 803

.043 or 828 or 833 or 837 or 841 or 845

.044 or 870 or 874 or 878 or 883 or 887

$\begin{array}{lllllllll}1.045 & \text {.or } 912 & \text {.or } 916 & \text {.or } 920 & \text {.or } 924 & \text {.or } 928\end{array}$

.046 or 953 or 957 or 961 or 966 or 970

$\begin{array}{llllllll}.047 & \text { or } 995 & \text { 01 } 999 & 02 & 003 & 02007 & 02011\end{array}$

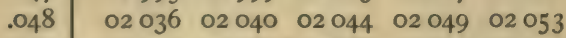

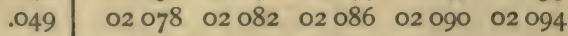

$\begin{array}{llllllll}00065 & 00069 & 00074 & 00078 & 00082\end{array}$ 0010800113001170012100126 $001520015600160 \quad 0016500169$ $0019500199 \quad 00204 \quad 00208 \quad 00212$ .00238 .00243 .00247 .00251 .00255 $\begin{array}{llllll}00281 & 00286 & 00290 & 00294 & 00 & 299\end{array}$ $00325 \quad 00329 \quad 00333 \quad 0337 \quad 00342$ 00 $368 \quad 00372 \quad 00376 \quad 00381 \quad 00385$ $\begin{array}{llllllll}00411 & 00415 & 00419 & 00424 & 00428\end{array}$

.00454 .00458 .00462 .00467 .00471 $0049700501 \quad 00505 \quad 0050900514$ $00540 \quad 00544 \quad 00548 \quad 00552 \quad 00557$ $00582005^{87} \quad 00591 \quad 00595 \quad 00600$ $00625 \quad 00629 \quad 00634 \quad 00638 \quad 00642$ .00668 .00672 .00677 .00681 .00685 $\begin{array}{lllllllll}00711 & 00715 & 00719 & 00724 & 00728\end{array}$ $007530075^{8} \quad 00762 \quad 00766 \quad 0077$ I $0079600800 \quad 00805 \quad 0080900813$ $00839 \quad 0843 \quad 00847 \quad 00852 \quad 00856$

.0088 1. .00886.00890.00894.00898 $00924 \quad 00928 \quad 00932 \quad 00937 \quad 0094 I$ $\begin{array}{lllllll}00966 & 00971 & 00975 & 00979 & 00983\end{array}$ or 009 or or 3 oror 7 or 022 or 026 or o5 I or 055 or o6o or 064 or 068 .or 094 . O1 098 .01 102 .OI IO6 .OI III OI 136 or I 40 or 144 or 149 or 153 or 178 or 182 or 187 or 191 or 195 or 220 OI 225 OI 229 or 233 or 237 or 263 or 267 or 27 I OI 275 or 280

.OI 305 .OI 309 .OI 31 3 .OI 317 .OI 322 or 347 or 35 I or 355 or 360 or 364 or 389 or 393 or 397 or 402 or 406 or 43 or 435 or 439 or 444 or 448 or 473 or 477 or 481 or 486 or 490 .OI 515 .Or 519 .01 523 .01 528 .01 532 or 557 or 56 I or 565 or 570 or 574 or 599 or 603 or 607 or 611 or 616 or 64 I or 645 or 649 or 653 or 657 or 682 or 687 or 691 or 695 or 699

.01 724 .Or 728 .O1 733 .OI 737 .01 741 or 766 or 770 or 774 or 778 or 783 or 808 or 812 or 816 or 820 or 824 or 849 or 853 or 858 or 862 or 866 or 89 r or 895 or 899 or 903 or 907 .or 932 or 937 .or 941 .or 945 .or 949 or 974 or 978 or 982 or 986 or 991

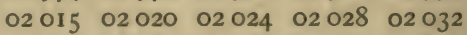

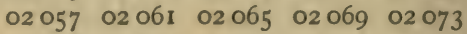
$\begin{array}{llllllll}02 & 098 & 02 & 102 & 02 & 107 & \text { O2 III } & \text { O2 II } 5\end{array}$ 


\begin{tabular}{|c|c|c|c|c|c|c|c|c|c|c|}
\hline No. & 0 & I & 2 & 3 & 4 & 5 & 6 & 7 & 8 & 9 \\
\hline 1.050 & .02119 & .02123 & .02127 & .02131 & .02135 & .02140 & .02144 & $.0214^{8}$ & .02152 & .02156 \\
\hline .051 & 02160 & 02164 & 02169 & 02173 & 02177 & 02181 & 02185 & 02189 & 02193 & 02197 \\
\hline .052 & 02202 & 02206 & 02210 & 02214 & 02218 & 02222 & 02226 & 02230 & 02235 & 02239 \\
\hline .053 & 02243 & 02247 & 02251 & 02255 & 02259 & 02263 & 02268 & 02272 & 02276 & 02280 \\
\hline .054 & 02284 & 02288 & 02292 & 02296 & 02301 & 02305 & 02309 & 02313 & 02317 & $0232 \mathrm{I}$ \\
\hline 1.055 & .02325 & .02329 & .02333 & $.0233^{8}$ & .02342 & .02346 & $.0235^{\circ}$ & .02354 & $.0235^{8}$ & .02362 \\
\hline .056 & 02366 & 02371 & 02375 & 02379 & $\mathrm{O}_{2} 3^{8} 3$ & $\mathrm{O}_{2} 3^{8} 7$ & 02391 & O2 395 & 02399 & 02403 \\
\hline .057 & 02407 & 02412 & 02416 & $02+20$ & 02424 & $02+28$ & 02432 & 02436 & $02+40$ & 02444 \\
\hline $.05^{8}$ & 02449 & 02453 & 02457 & 02461 & 02465 & 02469 & 02473 & 02477 & 02481 & $024 \$_{5}$ \\
\hline .059 & 02490 & 02494 & 02498 & 02502 & 02506 & 02510 & 02514 & 02518 & 02522 & 02526 \\
\hline 1.060 & .02531 & .02535 & .02539 & .02543 & .02547 & $.0255^{1}$ & .02555 & .02559 & $.025^{6} 3$ & .02567 \\
\hline .061 & 02572 & 02576 & $025^{80}$ & $025^{8} 4$ & 02588 & 02592 & 02596 & 02600 & 02604 & 02608 \\
\hline .062 & .02612 & 02617 & 02621 & 02625 & 02629 & 02633 & 02637 & 02641 & 02645 & 02649 \\
\hline .063 & 02653 & 02657 & 02661 & 02666 & 02670 & 02674 & 02678 & 02682 & 02686 & 02690 \\
\hline .064 & 02694 & 02698 & 02702 & 02706 & 02710 & 02715 & 02719 & 02723 & 02727 & 02731 \\
\hline 1.065 & .02735 & .02739 & .02743 & .02747 & $.0275^{1}$ & .02755 & .02759 & $.0276_{3}$ & .02768 & .02772 \\
\hline .066 & 02776 & 02780 & 02784 & 02788 & 02792 & 02796 & 02800 & $\mathrm{O}_{2} \mathrm{SO}_{4}$ & 02808 & $02 \mathrm{Si2}$ \\
\hline .067 & 02816 & $0282 I$ & 02825 & 02829 & 02833 & $0_{2} \$_{37}$ & $\mathrm{O}_{2} \mathrm{~S}_{41} \mathrm{I}$ & $028_{45}$ & 02849 & $028_{53}$ \\
\hline .068 & 02857 & $0286 \mathrm{r}$ & 02865 & 02869 & 02873 & 02877 & $\mathrm{O}_{2} \mathrm{SB}_{2}$ & 02886 & 02 Syo & 02894 \\
\hline .069 & 02898 & 02902 & 02906 & 02910 & 02914 & 02918 & 02922 & 02926 & 02930 & 02934 \\
\hline 1.070 & $.029 .3^{8}$ & .02942 & .02946 & $.0295^{I}$ & .02955 & .02959 & .02963 & .02967 & .02971 & .02975 \\
\hline .071 & 02979 & 02983 & 02987 & 02991 & 02995 & 02999 & $0_{3} 003$ & 03007 & $\mathrm{O}_{3}$ OII & 03015 \\
\hline .072 & 03019 & 03024 & 03028 & 03032 & 03036 & 03040 & $0_{3} 0_{44}$ & $\mathrm{O}_{3} \mathrm{O}_{4} \mathrm{~S}$ & $0_{3} 0_{5} 2$ & 03056 \\
\hline .073 & 03060 & 03064 & 03068 & 03072 & 03076 & 03080 & 03084 & 03088 & 03092 & 03096 \\
\hline .074 & 03100 & 03104 & 03109 & 03113 & 03117 & 03121 & 03125 & 03129 & 03133 & 03137 \\
\hline 1.075 & .03141 & .03145 & .03149. & .03153 & .03157 & .03161 & .03165 & .03169 & $.03 \times 73$ & .03177 \\
\hline .076 & 03181 & $03 I_{5}$ & O3 189 & 03193 & 03197 & 03201 & 03205 & 03.209 & 03214 & $\mathrm{O}_{3} 21 \mathrm{~S}$ \\
\hline .077 & $0_{3} 222$ & 03226 & 03230 & 03234 & 03238 & 03242 & 03246 & 03250 & 03254 & 03258 \\
\hline $.07^{8}$ & 03262 & 03266 & 03270 & 03274 & 03278 & 03282 & 03286 & $0_{3} 290$ & 03294 & 03298 \\
\hline .079 & 03302 & 03306 & 03310 & 03314 & 03318 & 03322 & 03326 & 03330 & O3334 & $\mathrm{O}_{3} 33^{\mathrm{S}}$ \\
\hline 1.080 & .03342 & .03346 & .03350 . & .03354 & $.0335^{8}$ & .03362 & .03366 & .03 .371 & .03375 & .03379 \\
\hline .081 & $0_{3} 3^{8} 3$ & $033^{8} 7$ & 03391 & O3 395 & 03399 & $0_{3} 40_{3}$ & 03407 & 03411 & 03415 & 03419 \\
\hline .082 & $0_{3423}$ & 03427 & 03431 & 03435 & 03439 & 03443 & $034+7$ & 03451 & 03455 & 03459 \\
\hline .083 & $0_{3} 463$ & 03467 & 03471 & 03475 & 03479 & $0_{3} 4_{3}$ & $0_{3} 487$ & 03491 & $0_{3} 495$ & 03499 \\
\hline .084 & 03503 & 03507 & 03511 & 03515 & 03519 & 03523 & 03527 & 03531 & O3535 & 03539 \\
\hline 1.085 & .03543 & .03547 & .03551. & .03555 & .03559 & .03563 & .03567 & .03571 & .03575 & .03579 \\
\hline .086 & $0_{35} 8_{3}$ & 03587 & 03591 & 03595 & 03599 & $\mathrm{O}_{3} \mathrm{WO}_{3}$ & 03607 & 03611 & $\mathrm{O}_{3} \mathrm{O}_{15}$ & 03619 \\
\hline .057 & 03623 & 03627 & $0_{3} 6_{31}$ & 03635 & 03639 & 03643 & 03647 & 03651 & 03655 & 03650 \\
\hline .088 & $\mathrm{O}_{3} 66_{3}$ & 03667 & 03671 & 03675 & 03679 & $\mathrm{O}_{3} 68_{3}$ & 03687 & 03691 & 03605 & $\mathrm{O}_{3}(x) \mathrm{x}$ \\
\hline .089 & 03703 & 03707 & 03711 & 03715 & 03719 & 03723 & 03727 & 03731 & 037.35 & 037,39 \\
\hline 1.090 & .03743 & .03747 & .03751 . & .03755 & .037 & $.037^{6} 3$. & $.037^{6} 7$ & .03771 & .03775 & $.037^{-3}$ \\
\hline .001 & $0_{3} 7^{82}$ & 03786 & 03790 & 03794 & 03798 & $\mathrm{O}_{3} \mathrm{So}_{2}$ & $0_{3} 506$ & O3S10 & $\mathrm{O}_{3} \mathrm{St}_{4}$ & $03 \mathrm{sis}$ \\
\hline .092 & $\mathrm{O}_{3} 822$ & 03826 & $0_{3} 830$ & $0_{3} 8_{34}$ & 03838 & $0_{3} 842$ & $o_{3} \delta_{46}$ & $0_{3} S_{50}$ & $0_{3} \$_{54}$ & $\mathrm{O}_{3} \mathrm{~S}_{5} \mathrm{~S}$ \\
\hline .093 & 03862 & 038166 & 03870 & $\mathrm{O}_{3} \mathrm{~S}_{74}$ & $0_{3} 878$ & $\mathrm{O}_{3} \mathrm{SS}_{2}$ & 03886 & $0_{3} 8 \times 0$ & $\left.0_{3} 51\right) 4$ & $0_{3} \mathrm{Sos}$ \\
\hline .094 & $0_{3} 902$ & 03906 & 03910 & 03914 & 03918 & 03922 & 03926 & 939.30 & 039,33 & 039.37 \\
\hline 1.095 & .03941. & .03945 & .03949 & .03953 & .03957 & .03961 & .03965 & $0_{3} g(x)$ & .03973 & .03977 \\
\hline .016 & $0308 i$ & $0_{3} 08_{5}$ & 03959 & 03993 & 03997 & 04001 & $04 \times 05$ & 04009 & $04 \mathrm{CI}_{3}$ & 04017 \\
\hline .097 & 04021 & 04025 & 04029 & 04033 & 04036 & 04040 & 04044 & 04045 & 04052 & 0.4056 \\
\hline .008 & 04060 & 04064 & 04063 & 04072 & 04076 & 0.4010 & 0408.4 & 04058 & 04002 & 04006 \\
\hline .099 & 04100 & 04104 & 04105 & 04112 & 04116 & 04120 & 0,1123 & 04127 & 04131 & $04 \quad 1.35$ \\
\hline
\end{tabular}




\section{1. FIVE PLACE LOGARITHMS.}

LOGS. 5 PL.

\begin{tabular}{|c|c|c|c|c|c|c|c|c|c|c|c|c|}
\hline No. & o & I & 2 & 3 & 4 & 5 & 6 & 7 & 8 & 9 & \multicolumn{2}{|c|}{$\begin{array}{l}\text { INTERPO. } \\
\text { TABLEB. }\end{array}$} \\
\hline \multirow[t]{5}{*}{1.00} & \multicolumn{10}{|c|}{$.00000 .00043 .00087 .00130 .00173 \quad .00217 .00260 .00303 .00346 .00389$} & \multirow{10}{*}{\multicolumn{2}{|c|}{$\begin{array}{c}\text { For } \log 1.0 \\
\text { to } \log 1.1 \\
\text { interpolated } \\
\text { values are } \\
\text { given on the } \\
\text { preceding } \\
\text { two pages. }\end{array}$}} \\
\hline & $\infty 432$ & $\infty 475$ & $\infty 5^{18}$ & $\infty 5^{61}$ & $0060_{4}$ & $\infty 647$ & & $\infty 732$ & 00775 & & & \\
\hline & $\infty 860$ & 00903 & $\infty 945$ & 00988 & 01030 & 01072 & OI 115 & or 157 & or 199 & 01242 & & \\
\hline & OI 284 & 01.326 & or 368 & 01410 & or $45^{2}$ & or 494 & or 536 & or 578 & OI 620 & 01662 & & \\
\hline & or 703 & or 745 & OI 787 & or 828 & or 870 & OI 912 & o1 953 & or 995 & 02086 & 02078 & & \\
\hline 1.05 & .02119 & .02160 & .02202 & .02243 & .02284 & .02325 & .02366 & .02407 & .02449 & .02490 & & \\
\hline .06 & 02531 & $0257^{2}$ & 02612 & 02653 & 02694 & 02735 & 02776 & 02816 & 02857 & O2 898 & & \\
\hline .07 & $0293^{8}$ & 02979 & 08019 & 03060 & 03100 & 03 I II & 03181 & 03222 & $\mathrm{O}_{3} 262$ & 03302 & & \\
\hline .08 & 03342 & 03383 & 03423 & 03463 & 03503 & 03543 & $0_{3} 5^{8} 3$ & 03623 & o 3663 & 03703 & & \\
\hline .09 & 03743 & 03782 & 03822 & 03862 & 03902 & $0394 \mathrm{I}$ & $0398 \mathbf{I}$ & 04021 & 04060 & 04100 & & \\
\hline 1.10 & .04 & .04 & .04218 & .042 & $.04=$ & .04336 & .04 & .04 & .04 & 3 & 38 & 36 \\
\hline .11 & 04532 & 04571 & 04610 & 04 & 04689 & 04727 & 04 & 04 & 04844 & $\mathrm{O}_{4} 88_{3}$ & 4 & 4 \\
\hline .12 & 04922 & 04961 & 04999 & 05038 & 05077 & 05115 & o5 154 & 05192 & 05231 & 05269 & 8 & 7 \\
\hline .13 & 05308 & 05346 & 05385 & 05423 & 05461 & 05500 & $0553^{8}$ & 05576 & 05614 & 05652 & II & II \\
\hline .14 & 05690 & 05729 & 05767 & 05805 & 05843 & $0588 \mathrm{I}$ & 05918 & 05956 & 05994 & 06032 & 15 & 14 \\
\hline I.1 5 & .06070 & $.06 x$ & .06 & .06 & .06221 & .06258 & .06 & .0 & & .06 & 19 & 18 \\
\hline .16 & 064 & 064 & 06 & 06 & 06 & 06 & 06 & 06 & 06 & $0678 \mathrm{I}$ & 23 & 22 \\
\hline .17 & 06819 & 06856 & 06893 & 069 & 06967 & 07004 & $0704 \mathrm{I}$ & 07078 & 07115 & 07151 & 27 & 25 \\
\hline .18 & 07188 & 07225 & 07262 & 07298 & o7 335 & 07372 & 07408 & 07445 & 07482 & 07518 & 30 & 29 \\
\hline .19 & 07555 & 07591 & 07628 & 07664 & 07700 & 07737 & 07773 & 07809 & 07846 & 07882 & 34 & 32 \\
\hline 1.20 & .07918 & & & & 63 & .08 & & & & & 34 & 33 \\
\hline .21 & 08279 & 08314 & 083 & 083 & 08 & $0845^{8}$ & 08 & & & & 3 & 3 \\
\hline .22 & 08636 & 08672 & 08707 & 08743 & $0877^{8}$ & 08814 & 08849 & 08884 & 08920 & 08955 & 7 & 7 \\
\hline .23 & 08991 & 09026 & 0906 I & 09096 & og 132 & 09167 & 09202 & 09237 & 09272 & 09307 & Io & 10 \\
\hline .24 & 09342 & 09377 & 09412 & 09447 & 09482 & 09517 & $0955^{2}$ & $095^{87}$ & 09621 & 09656 & 14 & I3 \\
\hline 1.25 & .09691 & .09726 & .09760 & .09 & .09830 & .09864 & .09899 & .09 & .09968 & .10003 & 17 & 17 \\
\hline .26 & 10037 & 10072 & 10106 & 10140 & 10175 & 10209 & 10243 & 10278 & 10312 & 10346 & 20 & 20 \\
\hline .27 & 10380 & 10415 & 10449 & I0 483 & 10517 & IO $55 \mathrm{I}$ & I0 $5^{85}$ & 10 619 & 10653 & I0 687 & 24 & 23 \\
\hline .28 & 10721 & 10755 & 10789 & 10823 & 10857 & Io 890 & 10924 & $1095^{8}$ & I0 992 & 11025 & 27 & 26 \\
\hline .29 & II 059 & II 093 & 11126 & 11160 & II 193 & II 227 & I 126 I & II 294 & II 327 & I I 361 & 31 & 30 \\
\hline 1.30 & .113 & .114 & & . I I & . II 528 & .11561 & & & & & 32 & 31 \\
\hline $.3 \mathrm{I}$ & 11727 & II 760 & 11793 & II 826 & 11 860 & II 893 & II & II & II 992 & 12024 & 3 & 3 \\
\hline .32 & 12057 & 12090 & 12123 & 12156 & 12189 & 12222 & 12254 & 12287 & 12320 & $1235^{2}$ & 6 & 6 \\
\hline .33 & 12385 & 12418 & $1245^{\circ}$ & 12483 & 12516 & $1254^{8}$ & $125^{8 \mathrm{I}}$ & 12613 & 12646 & 12678 & 10 & 9 \\
\hline .34 & 12710 & 12743 & 12775 & 12808 & 12840 & 12872 & 12905 & 12937 & 12969 & 13001 & I 3 & 12 \\
\hline 1.35 & .13033 & .13 & .13 & .13 & .13 & .13 & .13 & .13 & .1 & .1 & 16 & 16 \\
\hline .36 & I3354 & 13386 & 13418 & 13450 & 1348 I & 13513 & 13 & 13577 & 13609 & 13640 & 19 & 19 \\
\hline .37 & 13672 & 13704 & 13735 & 13767 & 13799 & 13830 & I3 862 & 13893 & I3925 & I $395^{6}$ & 22 & 22 \\
\hline $.3^{8}$ & 13988 & 14019 & 14051 & 14082 & $14114_{4}$ & 14145 & 14176 & 14208 & 14239 & 14270 & 26 & 25 \\
\hline .39 & 14301 & 14333 & 14364 & 14395 & 14426 & 14457 & 14489 & 14520 & $1455^{1}$ & $145^{82}$ & 29 & 28 \\
\hline 1.40 & .14613 & .146 & .14675 & .14 & .14 & .14768 & & .14829 & .14860 & .14891 & 30 & 29 \\
\hline $.4 \mathrm{I}$ & 14922 & 14953 & 14983 & 15014 & 15045 & I 5 ०76 & 15 & 15137 & 15168 & 15198 & 3 & 3 \\
\hline .42 & I5 229 & 15259 & 15290 & 15320 & I5 35I & $153^{81}$ & 15412 & 15 & 15473 & & 6 & 6 \\
\hline .43 & I5 534 & 15564 & I 5594 & 15625 & 15655 & 15685 & & 15 & & 15806 & 9 & 9 \\
\hline .44 & 15836 & 15866 & I 5897 & 15927 & 15957 & r 5987 & 16017 & 16.047 & 16077 & 16107 & 12 & 12 \\
\hline I. 45 & .16137 & .16167 & .16197 & .16227 & .16256 & .16286 & .16 & . 6346 & .16376 & .16406 & 15 & 15 \\
\hline .46 & 16435 & 16465 & 16495 & 16524 & I6 554 & I6 584 & 16 & 16643 & 16673 & 16702 & 18 & 17 \\
\hline .47 & 16732 & $1676 \mathrm{I}$ & 16791 & 16820 & 16850 & 16879 & 16909 & 16938 & 16967 & 16997 & 21 & 20 \\
\hline .48 & 17026 & 17056 & 17085 & $\mathrm{I}_{7} \mathrm{II}_{4}$ & 17143 & 17173 & 17202 & 17231 & 17260 & 17289 & 24 & 23 \\
\hline .49 & 17319 & 17348 & 17377 & I7 406 & I 7435 & 17464 & I7 493 & 17522 & $1755^{1}$ & $175^{80}$ & 27 & 26 \\
\hline
\end{tabular}




\begin{tabular}{|c|c|c|c|c|c|c|c|c|c|c|c|c|}
\hline No. & 0 & I & 2 & 3 & 4 & 5 & 6 & 7 & 8 & 9 & & \\
\hline 1.50 & .17609 & .17638 & .17667 & .17696 & .17725 & .17754 & $177^{82}$ & .17811 & .17840 & .17869 & 29 & 27 \\
\hline .51 & 17898 & 17926 & 17955 & 17984 & 18013 & $1 \mathrm{~S}_{41}$ & 18070 & 18099 & 18127 & 18156 & 3 & 3 \\
\hline .52 & 18184 & 18213 & 18241 & 18270 & 18298 & $1 S_{327}$ & 18355 & $188_{3} 4$ & 18412 & $1844 I$ & 6 & 5 \\
\hline .53 & 18469 & 18498 & $15 \leq 26$ & 18554 & 18583 & 18611 & 18639 & 18667 & 18696 & 18724 & 9 & 8 \\
\hline .54 & 18752 & 18780 & $18 \mathrm{Sod}$ & 18837 & 18865 & 18893 & 18921 & 18949 & 18977 & 19005 & 12 & II \\
\hline 1.55 & .19033 & $.1906 \mathrm{I}$ & .19089 & $.19 \times 17$ & .19145 & .19173 & .19201 & .19229 & .19257 & .19285 & 15 & 14 \\
\hline .56 & 19312 & 19340 & 19368 & 19396 & 19424 & 19451 & 19479 & 19507 & 19535 & 19562 & 17 & 16 \\
\hline .57 & 19590 & 19618 & 19645 & 19673 & 19700 & 19728 & 19756 & 19783 & 19811 & 19838 & 20 & 19 \\
\hline $.5^{8}$ & 19866 & 19893 & 19921 & 19948 & 19976 & 20008 & 20030 & $2005^{8}$ & 20085 & 20112 & 23 & 22 \\
\hline .59 & 20140 & $20 \times 67$ & 20194 & 20222 & 20249 & 20276 & 20303 & 20330 & $2035^{8}$ & 20385 & 26 & 24 \\
\hline 1.60 & .20412 & .20 & .204 & .20 & .20520 & .20548 & .20 & .20602 & .20629 & .20656 & 26 & 25 \\
\hline $.6 \mathrm{I}$ & 20683 & 20710 & 20737 & 20763 & 20790 & 20817 & 20844 & 20871 & 20898 & 20925 & 3 & 3 \\
\hline .62 & $2095^{2}$ & $2097^{8}$ & 21005 & 21032 & 21059 & 21085 & 21112 & 21139 & 21165 & 21192 & 5 & 5 \\
\hline .63 & 21219 & 21245 & 21272 & 21299 & 21325 & 21352 & $2137^{8}$ & 21405 & $2 I 43 I$ & $2145^{8}$ & 8 & 8 \\
\hline .64 & 21484 & $215^{11}$ & 21537 & 21564 & 21590 & 21617 & 21643 & 21669 & 21696 & 21722 & 10 & 10 \\
\hline 1.65 & $.2174^{8}$ & .21775 & .21801 & .21827 & .21854 & .21880 & .21906 & .21932 & .21 & .21985 & 13 & 13 \\
\hline .66 & 22011 & 22037 & 22063 & 22089 & 22115 & 22141 & 22167 & 22194 & 22220 & 22246 & 16 & 15 \\
\hline .67 & 22272 & 22298 & 22324 & 22350 & 22376 & 22401 & 22427 & 22453 & 22479 & 22505 & 18 & 18 \\
\hline .68 & 22531 & 22557 & $225^{8} 3$ & 22608 & 22634 & 22660 & 22686 & 22712 & 22737 & 22763 & 21 & 20 \\
\hline .69 & 22789 & 22814 & 22840 & 22866 & 22891 & 22917 & 22943 & 22968 & 22994 & 23019 & 23 & 23 \\
\hline 1.70 & .23045 & .23 & .23096 & $.23 \mathrm{I2I}$ & .23147 & .23 & .23198 & .23 & .23 & .23274 & 25 & 24 \\
\hline .71 & 23300 & 23325 & $2335^{\circ}$ & 23376 & 23401 & 23426 & $2345^{2}$ & 23477 & 23502 & 23528 & 3 & 2 \\
\hline .72 & 23553 & $2357^{8}$ & 23603 & 23629 & 23654 & 23679 & 23704 & 23729 & 23754 & 23779 & 5 & 5 \\
\hline .73 & 23805 & 23830 & 23855 & 23880 & 23905 & 23930 & 23955 & 23980 & 24005 & 24030 & 8 & 7 \\
\hline .74 & 24055 & 24080 & 24105 & $24 \times 30$ & 24155 & 24180 & 24204 & 24229 & 24254 & 24279 & 10 & 10 \\
\hline 1.75 & .24304. & .24329 & .24353 & .24378 & .24403 & .24428 & .24452 & .24477 & .24502 & .24527 & 13 & 12 \\
\hline .76 & 24551 & $2457^{6}$ & 24601 & 24625 & 24650 & 24674 & 24699 & 24724 & 24748 & 24773 & 15 & 14 \\
\hline .77 & 24797 & 24822 & 24846 & 24871 & 24895 & 24920 & 249.44 & 24969 & 24993 & 25018 & 18 & 17 \\
\hline .78 & 25042 & 25066 & 25091 & 25115 & 25139 & 25164 & 25188 & 25212 & 25237 & 25261 & 20 & 19 \\
\hline .79 & 25285 & 25310 & 25334 & $2535^{8}$ & 25382 & 25406 & 25431 & 25455 & 25479 & 25503 & 23 & 22 \\
\hline 1.80 & .255 & .25 & .25 & .25 & .25624 & .25648 & .25 & .25696 & & & 24 & 23 \\
\hline .81 & 25768 & 25792 & 25816 & 25840 & 25864 & 25888 & 25 & 25935 & 25 & 25983 & 2 & 2 \\
\hline.$\$ 2$ & 26007 & 26031 & 26055 & 26079 & 26102 & 26126 & 26150 & 26174 & 26198 & 26221 & 5 & 5 \\
\hline .83 & 26245 & 26269 & 26293 & 26316 & 26340 & 26364 & $263^{87}$ & $264^{11}$ & 26435 & $2645^{8}$ & 7 & 7 \\
\hline .84 & 26482 & 26505 & 26529 & 26553 & 26576 & 26600 & 26623 & 26647 & 26 & 26694 & 10 & 9 \\
\hline $1.8_{5}$ & .26717 & .26741 & .267 & .26788 & .26811 & .26834 & .26 & $.2688 \mathrm{I}$ & .26 & .26928 & 12 & 12 \\
\hline .86 & $2695 \mathrm{~L}$ & & 260 & 27021 & 270 & & 27091 & & & 27 & 14 & 14 \\
\hline .87 & 27184 & 27207 & 27231 & 27254 & 27277 & 27300 & 27.323 & 27346 & 27370 & 27393 & 17 & 16 \\
\hline ss & 27416 & 27439 & 27462 & 27485 & 27508 & $2753^{1}$ & 27554 & 27577 & $\infty$ & 27623 & 19 & 18 \\
\hline 80 & 27646 & 27669 & 27692 & 27715 & $2773^{8}$ & 27761 & $277^{84}$ & 27507 & 27 & 27852 & 22 & 20 \\
\hline 1.90 & .27875 & .27 & .27921. & .279 & .27967 & .27989 & .28012 & $.28 \mathrm{C}$ & 58. & $.2808 \mathrm{i}$ & 28 & 21 \\
\hline .91 & 28103 & 28126 & 28149 & 28171 & 28194 & 28217 & 28240 & 28262 & 28285 & 28307 & 2 & 2 \\
\hline .92 & 28330 & 28353 & 28375 & 28398 & 28421 & 28443 & 28466 & 28488 & 28511 & 28533 & 4 & 4 \\
\hline .93 & 28556 & $2857^{8}$ & 28601 & 28623 & 28646 & 28668 & $2869 x$ & $287^{13}$ & 28735 & 28758 & 7 & 6 \\
\hline .94 & 28780 & 28803 & 28825 & 28847 & 28870 & 28892 & 28914 & 289.37 & 28959 & 28981 & 9 & 8 \\
\hline .95 & .29008 & .29026 & .29048. & .29070 & .29092 & .29815 & .29837 & .29159 & .29181. & .29203 & 11 & 11 \\
\hline & 29226 & 29248 & 29270 & 29292 & 29314 & 29336 & $2935^{8}$ & 29) 350 & 29403 & 29425 & 13 & 83 \\
\hline .97 & 29447 & 29469 & 29491 & 29513 & 29535 & 29557 & 29579 & $20(x) 1$ & 29623 & 29645 & 15 & 15 \\
\hline & 29667 & 29688 & 29710 & 29732 & 29754 & 29776 & 29798 & 39820 & $29 \mathrm{~S}_{42}$ & 29863 & 18 & 17 \\
\hline .99 & 29885 & 29907 & 29929 & 29951 & 29973 & 29994 & 30016 & 30038 & 30060 & 30081 & 20 & 19 \\
\hline
\end{tabular}


LOGS. 5 PL.

\begin{tabular}{|c|c|c|c|c|c|c|c|c|c|c|c|}
\hline No. & 0 & I & 2 & 3 & 4 & 5 & 6 & 7 & 8 & 9 & $\begin{array}{l}\text { NTP. } \\
\text { ABB. }\end{array}$ \\
\hline .00 & 30 103 & .30125 & .30146 & & .30 & II & .30233 & .30 & .30276 & .30298 & 21 \\
\hline & 30320 & $3034 \mathrm{I}$ & $303^{6} 3$ & 30384 & & & & & & & \\
\hline .02 & 30535 & 30557 & 30578 & 30600 & 30621 & & & & & & \\
\hline .03 & 30750 & $3077^{1}$ & 30792 & 30814 & 30835 & 30856 & 30878 & 30899 & & & \\
\hline .04 & 30963 & 30984 & 81006 & 31027 & & 31069 & 31091 & 31112 & 31133 & $\begin{array}{ll}31 & 154\end{array}$ & 8 \\
\hline 2.05 & .31175 & .31197 & .31218 & .31239 & .31260 & $.3128 I$ & .31302 & .31323 & .31345 & .31366 & II \\
\hline & $3^{1} 3^{87}$ & 31408 & 31429 & $3145^{\circ}$ & 31 & 31492 & 31 & & & & 13 \\
\hline .07 & $3^{1} 597$ & 31618 & 31639 & 31660 & 3 I 68I & & & & & & 15 \\
\hline .08 & 31806 & 31 827 & 31848 & 31869 & 31890 & $3^{1} 911$ & 31931 & & 31973 & & 17 \\
\hline .09 & 32015 & 32035 & 32056 & 32077 & 32098 & 32118 & 32139 & 32 & 32181 & & 19 \\
\hline 2.10 & .32 & .32 & & $\cdot 32$ & & & & & .32 & & 20 \\
\hline & 32428 & $3^{24}$ & 32469 & 32 & & 32 & 32 & & & & \\
\hline & 32634 & 32654 & 32675 & 32695 & $3^{2}$ & 32 & 32 & & & & \\
\hline .13 & 328 & $3285^{8}$ & 32879 & 328 & $3^{2}$ & $3^{2}$ & 32960 & 32 & 33 & & \\
\hline .14 & 33041 & 33062 & 33 & 33 & 33 & 33 & 33163 & 33 & 33 & & \\
\hline 2.15 & .33244 & $\cdot 33$ & .33 & .33 & & & & & & & 10 \\
\hline 16 & 334 & 334 & 33 & 33 & & & & & & & 12 \\
\hline 7 & 336 & 336 & 33 & 33 & 33 & & & & & & 14 \\
\hline .18 & 33846 & 33866 & 33885 & 33 & 33 & 33 & 33 & 33 & & & 16 \\
\hline .19 & 34044 & 34064 & . 34084 & 34 & 34 & 3 & 63 & 34 & 34 & & 18 \\
\hline 20 & .34242 & .34 & & & & & & & & & 19 \\
\hline & 344 & 34 & & & & & & & & & 2 \\
\hline .22 & 346 & $34 \epsilon$ & 34 & 34 & & & & & & & \\
\hline .23 & 34830 & 348 & 34 & 34 & & & & & & & \\
\hline & & & & & & & & & & & 8 \\
\hline 2.25 & .35218 & .35238 & .35 & $\cdot 3$ & & & & & & & 10 \\
\hline & $354 \mathrm{II}$ & 35430 & & & & & & & & & 1 \\
\hline & 35603 & 356 & & 35 & & & & & & & 3 \\
\hline .28 & 35793 & 35813 & $35^{832}$ & $3585^{1}$ & 35 & 39 & 35 & & & & 15 \\
\hline .29 & 35984 & & 36021 & & & & & & 36 I 35 & 36 & 17 \\
\hline 2.30 & & & & & & & & & & & 18 \\
\hline 131 & 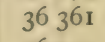 & -363 & 36 & 36 & & & & & & & 0 \\
\hline .32 & 365 & 365 & $3^{6} 5^{86}$ & & & & & & & & 4 \\
\hline .33 & 3 & 367 & 36773 & & 36 & & & & & & \\
\hline .34 & 3 & $36 c$ & 36 & 77 & 36 & 4 & 3 & & & & 7 \\
\hline 2.35 & $\cdot 3$ & $\cdot 3$ & & 2 & & & 218 & & & & 9 \\
\hline & 37291 & & & 37 & & & & & & & I \\
\hline .37 & & 37 & & 37 & & & 3 & & & & 13 \\
\hline & & & & & & & & & & & 14 \\
\hline .39 & 37840 & 37 & 37 & 37 & 37 & 3 & $3 \%$ & 37 & & 38003 & 16 \\
\hline 2.40 & .38021 & $\cdot 3$ & & & & & & & & & 17 \\
\hline $.4 \mathrm{I}$ & & & & $3^{8} 25^{6}$ & & & & & & & 2 \\
\hline .42 & $3^{8} 382$ & 38399 & & & & & & & & & 3 \\
\hline .43 & $3^{8} 5^{61}$ & $3^{8} 57^{8}$ & $3^{8} 596$ & & & & & & & & \\
\hline .44 & 38739 & 38757 & 38775 & $3^{8} 792$ & 38810 & & 46 & $3^{8}$ & & & 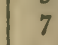 \\
\hline 2.45 & .38917 & .38934 & $.3895^{2}$ & $.3^{8} 970$ & & .39 & .39 & .39041 & $.3905^{8}$ & & \\
\hline .46 & 39094 & 39 I I I & 39129 & 39146 & & & & & & & 10 \\
\hline .4 & 39270 & & & & & & & & & & 12 \\
\hline 40 & & 39463 & & & & & & & & & 14 \\
\hline .49 & 39620 & 39637 & 39655 & 39672 & 39690 & 39707 & 39724 & 39742 & 39759 & 39777 & 15 \\
\hline
\end{tabular}




\begin{tabular}{|c|c|c|c|c|c|c|c|c|c|c|c|}
\hline No. & 0 & I & 2 & 3 & 4 & 5 & 6 & 7 & 8 & 9 & $\begin{array}{l}\text { INTP. } \\
\text { TAB. }\end{array}$ \\
\hline 2.50 & .39794 & .39811 & .39829 & .39846 & $.39 \mathrm{Só}_{3}$ & $.3988 \mathbf{I}$ & .39 898 & & & .39950 & 18 \\
\hline .51 & 39967 & 39985 & 40002 & 40019 & 40037 & 40054 & 40071 & 40058 & & 40123 & 2 \\
\hline .52 & 40140 & 40157 & 40175 & 40192 & 40209 & 40226 & 40243 & 40261 & 40278 & 40295 & 4 \\
\hline .53 & 40312 & 40329 & 40346 & 40364 & $403^{8 I}$ & 40398 & 40415 & 40432 & $40+49$ & 40466 & 5 \\
\hline .54 & 40483 & 40500 & 40518 & 40535 & $4055^{2}$ & 40569 & $405^{86}$ & 40603 & 40620 & 40637 & 7 \\
\hline 2.55 & 40654 & .40671 & .40688 & .40705 & .40722 & .40739 & .40756 & .40773 & .40790 & .40807 & 9 \\
\hline .50 & 40824 & 40841 & 40858 & 40875 & $40 S 92$ & 40909 & 40926 & 40943 & 40 & 40976 & II \\
\hline .57 & 40993 & 41010 & 41027 & 41044 & 41061 & $4^{1} 078$ & 41095 & 41111 & 41128 & 41145 & 13 \\
\hline $.5^{8}$ & 41162 & 41179 & 41196 & 41212 & 41229 & 41246 & $\begin{array}{lll}41 & 263\end{array}$ & $412 S O$ & 41296 & 41313 & 14 \\
\hline$\cdot 59$ & 41330 & $4^{1} 347$ & $4^{1} 363$ & $4^{1} 350$ & 41397 & 41414 & 41430 & 41447 & 41464 & $4 I 48 I$ & 16 \\
\hline 2.60 & 41497 & 41514 & .41531 & .41547 & -41564 & $.415^{81}$ & .41597 & .41614 & .41631 & .41647 & 17 \\
\hline .61 & 41664 & 41681 & 41697 & 41714 & 41731 & 41747 & 41764 & 41780 & 41 & 14 & 2 \\
\hline .62 & 41830 & 41847 & 41863 & 41880 & 41896 & $4^{1} 9^{1} 3$ & 41929 & 41946 & 41963 & 41979 & 3 \\
\hline .63 & 41996 & 42012 & 42029 & 42045 & 42062 & 42078 & 42095 & 42111 & 42127 & 42144 & 5 \\
\hline .64 & 42160 & 42177 & 42193 & 42210 & 42226 & 42243 & 42259 & 42275 & 42292 & 42308 & 7 \\
\hline 2.65 & .42325 & .42341 & .42357 & .42374 & .42390 & .42406 & .42423 & .42439 & .42455 & $.4247^{2}$ & 9 \\
\hline .66 & $424 S S$ & 42504 & $4^{2} 521$ & 42537 & $4^{2} 553$ & 42570 & $425^{86}$ & 42602 & 42619 & 35 & 10 \\
\hline .67 & 42651 & 42667 & 42684 & 42700 & 42716 & 42732 & 42749 & 42765 & $4279 I$ & 42797 & 12 \\
\hline .68 & 42813 & $428_{30}$ & $42 S_{46}$ & 42862 & $42 S_{7} \delta$ & 42894 & 42911 & 42927 & 42943 & 42959 & 14 \\
\hline .69 & 42975 & 42991 & 43008 & 43024 & 43040 & 43056 & 43072 & 43088 & 43104 & 43120 & 15 \\
\hline 2.70 & .43136 & .43152 & .43 & .43185 & .432 & .43 & .43 & & & 1 & 16 \\
\hline .71 & 43297 & 43313 & 43329 & 43345 & 433 & 4 & 43393 & 43 & 43 & 41 & 2 \\
\hline .72 & 43457 & 43473 & 43489 & 43505 & 435 & 4. & 43553 & 43569 & $435^{84}$ & 00 & 3 \\
\hline .73 & 43616 & 43632 & 43648 & 43664 & 43680 & 43 & $437^{12}$ & 43727 & 43743 & 43759 & 5 \\
\hline .74 & 43775 & 43791 & $43 \mathrm{SO} 7$ & 43823 & 43838 & 43854 & 43870 & 43886 & 43902 & 43917 & 6 \\
\hline 2.75 & .43933 & .43949 & .43965 & .43981 & .43996 & .44 & .44 & .44 & 059. & .44075 & 8 \\
\hline & 44091 & 44107 & 44122 & $44^{1} 3^{8}$ & $44^{154}$ & 44170 & 44185 & 44201 & 44217 & 32 & 10 \\
\hline-77 & 442.48 & 44264 & 44279 & 44295 & 44311 & 44326 & 44342 & $4435^{8}$ & 44373 & $\left.443^{S}\right)$ & II \\
\hline .78 & 44404 & 44420 & 44436 & $4445^{I}$ & 44467 & $444^{8} 3$ & 44498 & $445^{14}$ & 44529 & 44545 & 13 \\
\hline-0 & 44560 & $4457^{6}$ & 44592 & 44607 & 44623 & 44638 & 44654 & 44669 & 44685 & 44 & 14 \\
\hline 80 & .44716 & .44731 & .44 & $.447^{62}$ & $.4477^{8}$ & & .44 & .44 & 40. & 55 & 15 \\
\hline 81 & $44 S_{71}$ & 44886 & 44902 & 44917 & 44932 & 4 & 44963 & 44 & 94 & 10 & 2 \\
\hline .82 & 45025 & 450.40 & 45056 & 45071 & 45086 & 45 & 45 & 45 & 45 & 3 & 3 \\
\hline .83 & $45 \quad 179$ & 45194 & 45209 & 45225 & 45240 & 45 & 45271 & 45286 & 45 & & 5 \\
\hline 34 & 45332 & 45347 & 45362 & $4537^{8}$ & 45393 & 45408 & $454^{23}$ & 45439 & 45454 & 45469 & 6 \\
\hline 85 & .45484 & .45500 & .45515 & .45530 & .45 & .45561 & .45576 & .45591 & .45606 & .45621 & 8 \\
\hline & 45637 & 45652 & 45667 & 45682 & 45697 & 45 & 45 & 45743 & $4575^{8}$ & 773 & 9 \\
\hline $8_{7}$ & 45758 & $45 \mathrm{SO}_{3}$ & 45818 & ${ }_{45} \delta_{34}$ & 45849 & 45864 & 45879 & $45 \mathrm{~S}\left({ }_{4}\right.$ & 45 & 24 & 11 \\
\hline .85 & 45939 & 45954 & 45969 & 45,984 & 46000 & 46015 & 460,30 & 46045 & 460100 & 46075 & 12 \\
\hline .89 & $4^{6} 050$ & $4^{6} 105$ & 46120 & 46135 & 46150 & 46165 & 461 So & 46195 & 10 & 25 & 14 \\
\hline 2.90 & $.46,240$ & .46255 & .46270 & .46285 & .40 & & .46330 & 45 & 59. & 74 & 14 \\
\hline .91 & 46389 & 46404 & 46419 & 464.34 & 46449 & 46464 & 46479 & 46494 & $465(x)$ & 46523 & 1 \\
\hline .92 & 46538 & $4^{6} 553$ & 46568 & $4^{6} 5^{5} 3$ & $4^{6} 508$ & 46613 & 46627 & $4^{6} 6.42$ & 46657 & 40672 & 3 \\
\hline .93 & 46687 & $4^{6} 7^{02}$ & 46716 & 46731 & 46746 & $4^{6} 7^{6} 1$ & 46776 & $467(x)$ & $46 \mathrm{SO}_{5}$ & 46520 & 4 \\
\hline .94 & 46835 & 46850 & 46864 & 46879 & 46894 & 46909 & 46923 & 46938 & 46953 & 46967 & b) \\
\hline 95 & $.469 \$_{2}$ & 46997 & .47012 & .47026 & .47041 & 47056 & 47070 & $470 S_{5}$ & .47100 & 47 & 7 \\
\hline .96 & 47129 & 47144 & $47+59$ & 47173 & 47158 & 47202 & 47217 & 47232 & $4724^{6}$ & 47261 & 8 \\
\hline .97 & $4727^{6}$ & 472.00 & 47305 & 47319 & 47334 & 47349 & $473^{6} 3$ & $4737^{8}$ & 47392 & 47407 & 10 \\
\hline .98 & 47422 & $474.3^{6}$ & 47451 & 47465 & 47450 & 47494 & 47509 & 47524 & 47538 & & II \\
\hline .99 & 47567 & $475^{82}$ & 47596 & 47611 & 47625 & 47640 & 47654 & 47669 & $4768_{3}$ & $47 \mathrm{cogs}$ & 13 \\
\hline
\end{tabular}




\section{3. \\ FIVE PLACE LOGARITHMS.}

LOGS. 5 PL.

\begin{tabular}{|c|c|c|c|c|c|c|c|c|c|c|c|}
\hline No. & 0 & I & 2 & 3 & 4 & 5 & 6 & 7 & 8 & 9 & $\begin{array}{l}\text { INTP. } \\
\text { TAB. }\end{array}$ \\
\hline 3.00 & .47712 & .47727 & .47741 & .47756 & .47770 & .47784 & .47799 & .47813 & .47828 & .47 & 15 \\
\hline .01 & & 478.71 & $478_{5}$ & 47900 & 47914 & 47929 & 47943 & $4795^{8}$ & 47972 & & 2 \\
\hline .02 & 48001 & 48 OI 5 & 48029 & 48044 & $48 c$ & 48073 & $48 \circ 87$ & 48 IOI & 48 I I 6 & & 3. \\
\hline .03 & $48 \times 44$ & 48 I 59 & $48 \quad 173$ & 48187 & 48202 & 48216 & 48230 & 48244 & 48259 & 48273 & \\
\hline .04 & 48287 & $4^{8} 302$ & $4^{8} 316$ & $4^{8} 330$ & $4^{8} 344$ & 48359 & $4^{8} 373$ & $4^{8} 3^{87}$ & & 48416 & 0 \\
\hline 3.05 & .48430 & .48444 & $.4^{8} 45^{8}$ & .48473 & $.484^{87}$ & .48501 & .48515 & .48530 & .48544 & $.4^{8} 55^{8}$ & 8 \\
\hline .06 & $4^{8} 57^{2}$ & $4^{8} 5^{86}$ & 48601 & 48615 & 48 & 48643 & 48657 & 48671 & & 48700 & 9 \\
\hline .07 & 48714 & 48728 & 48742 & 48756 & $4^{8} 770$ & 48785 & 48799 & 48813 & 48827 & 48841 & II \\
\hline .08 & 48855 & 48869 & 48883 & 48897 & 489 II & 48926 & 48940 & 48954 & 48968 & 48982 & 12 \\
\hline .09 & 48996 & 49010 & 49024 & 49038 & 49052 & 49066 & 49080 & 49094 & $\begin{array}{ll}49108\end{array}$ & 49122 & 14 \\
\hline 3.10 & .49136 & .49150 & $.49 I 64$ & .49178 & .49192 & .49206 & .49220 & .49 & .49248 & .49262 & 14 \\
\hline .11 & 49276 & 49290 & 49304 & 49318 & 49 & 49346 & 49360 & 49 & & & I \\
\hline .12 & 49415 & 49429 & 49443 & 49457 & 49 & 49485 & 49 & 49 & & & 3 \\
\hline .13 & 49554 & 49568 & $495^{82}$ & 49 & 49 & 49624 & 49 & 49 & & & 4 \\
\hline .14 & 496 & 49707 & 49721 & 49734 & 497 & 49 & 49776 & 49 & 49803 & $498 \mathrm{I} 7$ & 0 \\
\hline 3.15 & .49831 & .49845 & .49859 & .49872 & .49 & .49900 & .49 & .49 & .49 & .49 & \\
\hline .16 & 49969 & 49982 & 49996 & 50010 & 50 & 50 & & & & & 8 \\
\hline .17 & 50106 & 50120 & 50133 & 50147 & 50 & 50 & 50188 & 50202 & & & 0 \\
\hline .18 & 50243 & 50256 & 50270 & 50284 & 50297 & 50 & 50325 & 50338 & 50 & 5 & 1 \\
\hline 19 & 50379 & $5^{\circ} 393$ & 50406 & 50420 & 50 & & & 50474 & & & 3 \\
\hline 3.20 & .5 & .505 & .505 & .50556 & .5 & 3 & & 10 & $\cdot 5$ & $\cdot 5$ & 13 \\
\hline .21 & 50651 & 50 & 50 & 50 & 50 & 50 & 50 & & & & I \\
\hline .22 & 50786 & 50799 & 508 & 50 & $5^{\circ}$ & 50 & 50 & & & 50 & 3 \\
\hline .23 & 50920 & 50934 & 50947 & 50961 & 50 & 505 & 51 & $5^{I}$ & & $5^{1}$ & 4 \\
\hline .24 & 51055 & 51068 & 51081 & $5^{1} 095$ & $5^{1}$ & $5112 I$ & $5^{1} 135$ & $5^{11} 14^{8}$ & 51162 & 51175 & 5 \\
\hline 3.25 & .51188 & .51202 & .51215 & .51228 & .51242 & .51255 & .51268 & .51282 & .51295 & .51308 & 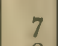 \\
\hline .26 & 51322 & $5^{1} 3$ & $5^{1} 34^{8}$ & $5^{1}$ & $5^{I}$ & & 5 & 15 & & 51 & \\
\hline .27 & $5^{1} 455$ & $5^{1} 4$ & $5^{1} 4^{81}$ & $5^{1} 495$ & $5^{1}$ & I & $5^{1}$ & $5^{1} 54^{8}$ & & $5^{I}$ & 9 \\
\hline .28 & $5^{1} 5^{87}$ & $5^{1} 601$ & 51614 & $5^{1} 627$ & 5 I & 51654 & $5^{I}$ & & $5^{I}$ & $5^{1} 706$ & 10 \\
\hline .29 & $5^{1} 720$ & 51733 & $5^{\text {I. } 746}$ & 51759 & $5177^{2}$ & $5^{1} 786$ & & 51812 & 51825 & $5^{1838}$ & 2 \\
\hline 3.30 & .51851 & .51865 & .518 & $.5^{1}$ & .5 & 7 & & & & & 13 \\
\hline $.3 \mathrm{I}$ & $5^{1} 983$ & $5^{1} 996$ & 52009 & 52022 & $5^{2}$ & 52048 & 52 & & & & I \\
\hline .32 & 52114 & 52127 & 52140 & $52 \times 53$ & 52166 & 52179 & 52 & $5^{2}$ & & & 3 \\
\hline .33 & 52244 & 52257 & 52270 & 52284 & 522 & $5^{2} 3$ & $5^{2}$ & $5^{2}$ & & & $T$ \\
\hline$\cdot 34$ & $5^{2} 375$ & $5^{2} 3$ & 52401 & 52414 & 52427 & $5^{2} 440$ & $5^{2} 453$ & 52466 & $5^{2} 479$ & $5^{2} 492$ & 5 \\
\hline 3.35 & .52504 & $.5^{2}$ & .52530 & $.5^{2} 543$ & .52556 & .52569 & $.5^{2} 5^{82}$ & .5 & .52608 & 1 & \\
\hline 6 & $5^{2} 634$ & & 52660 & 52673 & $5^{2}$ & $5^{2}$ & & & & & \\
\hline 37 & $5^{2} 763$ & 527 & $5^{2} 789$ & 52802 & 52 & $5^{2}$ & $5^{2}$ & & & & 9 \\
\hline$\cdot 38$ & 52892 & 52905 & 52917. & 52930 & $5^{2} \mathrm{~s}$ & 529 & 52969 & $5^{2}$ & & 53 & 10 \\
\hline .39 & 53020 & $53 \circ 33$ & 53046 & 53058 & 53071 & 53084 & 53097 & 53110 & 53122 & 53135 & 12 \\
\hline 3.40 & & & & & & & & & & & 12 \\
\hline $.4 I$ & 53275 & 53 & & 53 & 533 & & 53 & 53364 & & 53390 & I \\
\hline .42 & 53403 & 53415 & 53428 & 53441 & 53453 & 53466 & 53479 & 53491 & & 53517 & 2 \\
\hline .43 & 53529 & 53542 & 53555 & 53567 & 535 & 53593 & 53605 & 53618 & 53 & & 4 \\
\hline .44 & 53656 & 536 & $5368 I$ & 53694 & 53706 & 53719 & 53732 & 53744 & 53757 & 53769 & $J$ \\
\hline 3.45 & .53782 & .53794 & .53807 & .53820 & .53832 & .53845 & .53857 & .53870 & & .53895 & 6 \\
\hline .46 & 53908 & 53920 & 53933 & 53945 & $5395^{8}$ & 53970 & 53983 & 53995 & 54008 & 54020 & \\
\hline .47 & 54033 & 54045 & 54058 & 54070 & 54083 & 54095 & 54108 & 54120 & 54133 & $54 \mathbf{I} 45$ & \\
\hline .48 & $5415^{8}$ & $54 I 70$ & 54183 & 54195 & 54208 & 54220 & 54233 & 54245 & $5425^{8}$ & 54270 & Io \\
\hline .49 & 54283 & 54295 & 54307 & 54320 & $5433^{2}$ & 54345 & 54357 & 54370 & $543^{82}$ & 54394 & II \\
\hline
\end{tabular}




\begin{tabular}{|c|c|c|c|c|c|c|c|c|c|c|c|}
\hline No. & 0 & I & 2 & 3 & 4 & 5 & 6 & 7 & 8 & 9 & $\begin{array}{l}\text { INTP. } \\
\text { TAB. }\end{array}$ \\
\hline 3.50 & .54407 & .54419 & .54432 & .54444 & .54456 & .54469 & .54481 & .54494 & .54506 & .54518 & 13 \\
\hline .51 & 54531 & 54543 & 54555 & 54568 & 54580 & 54593 & 54605 & 54617 & 54630 & 54642 & I \\
\hline$-5^{2}$ & 54654 & 54667 & 54679 & 54691 & 54704 & 54716 & 54728 & $5474 I$ & 54753 & 54765 & 3 \\
\hline .53 & 54777 & 54790 & 54802 & 54814 & 54827 & 54839 & $5485^{1}$ & 54864 & 54876 & & 4 \\
\hline .54 & 54900 & 54913 & 54925 & 54937 & 54949 & 54962 & 54974 & 54986 & 54998 & 55011 & 5 \\
\hline 3.55 & .55023 & .55035 & .55047 & .55060 & .55072 & .55084 & .55096 & $.55 \quad 108$ & .55121 & $.55 \mathrm{I} 33$ & 7 \\
\hline .56 & 55145 & $55 \quad 157$ & $55^{169}$ & 55182 & 55194 & 55206 & 55218 & 55230 & $55^{242}$ & 55 & 8 \\
\hline .57 & 55267 & 55279 & 55291 & 55303 & 55315 & 55328 & 55340 & $5535^{2}$ & 55364 & 55376 & 9 \\
\hline $.5^{8}$ & $553^{88}$ & 55400 & 55413 & 55425 & 55437 & 55449 & 55461 & 55473 & 55485 & 55497 & 10 \\
\hline .59 & 55509 & $555^{22}$ & 55534 & 55546 & $5555^{8}$ & 55570 & $555^{82}$ & 55594 & 55606 & 55618 & 12 \\
\hline 3.60 & .55630 & .55642 & .55654 & .55666 & .55678 & .55691 & .55703 & .55 & .55 & .55739 & 12 \\
\hline .61 & 55751 & 55763 & 55775 & $557^{8} 7$ & 55799 & $55 \mathrm{SII}$ & 55823 & 55835 & 55847 & 55 & I \\
\hline .62 & 55871 & $55 \mathrm{Ss}_{3}$ & 55895 & 55907 & 55919 & 55931 & 55943 & 55955 & 55967 & 55 & 2. \\
\hline .63 & 55991 & 56003 & $5^{6} 015$ & 56027 & 56 o38 & 56050 & 56062 & 56074 & 56086 & & 4 \\
\hline .64 & 56110 & 56122 & 56 I34 & 56146 & $5615^{8}$ & 56170 & 56182 & 56194 & 56205 & 56217 & 5 \\
\hline 3.65 & .56229 & $.5624 \mathrm{I}$ & .56253 & .56265 & .56277 & .56289 & .56301 & .56312 & .56324 & .56336 & 6 \\
\hline .66 & $5^{6} 34^{8}$ & 56360 & 56372 & $5^{6} 3^{84}$ & 56396 & 56407 & 56419 & $5^{6} 431$ & 56443 & 55 & 7 \\
\hline .67 & 56467 & 56478 & 56490 & 56502 & 56514 & 56526 & $5653^{8}$ & 56549 & 56561 & 56573 & 8 \\
\hline .68 & $5^{6} 5^{8}$ & 56597 & 56608 & 56620 & 56632 & 56644 & 56656 & 56667 & 56679 & 56691 & 10 \\
\hline .69 & 56703 & 56714 & 56726 & $5^{6} 73^{8}$ & $5^{6} 750$ & 56761 & 56773 & $5^{6} 785$ & 56797 & 56 & II \\
\hline 3.70 & .56820 & .56832 & .56 & .56 & .56 & .56 & 1 & .56 & .56914 & .56926 & 12 \\
\hline .71 & $5^{6} 937$ & 56949 & $5696 \mathrm{I}$ & 56972 & 56984 & 56996 & 57008 & 57019 & $57 \circ 31$ & 57043 & I \\
\hline$\cdot 7^{2}$ & 57054 & 57066 & $57^{078}$ & 57089 & 57 IOI & 57113 & 57124 & 57136 & 57148 & 57 & 2 \\
\hline .73 & 57171 & $57 \times 8_{3}$ & 57194 & 57206 & 57217 & 57229 & 57241 & 57252 & 57264 & 57276 & 4 \\
\hline .74 & 57287 & 57299 & 57310 & 57322 & 57334 & $57345^{\circ}$ & 57357 & 57368 & $573^{80}$ & 57392 & 5 \\
\hline 3.75 & .57403 & .57415 & .57426 & $.5743^{8}$ & .57449 & $.5746 \mathrm{I}$ & .57473 & .57484 & .57496 & .57507 & 6 \\
\hline $.7^{6}$ & 57519 & 57530 & 57542 & 57553 & 57565 & $5757^{6}$ & 57588 & 57600 & 57611 & 57623 & 7 \\
\hline .77 & 57634 & 57646 & 57657 & 57669 & 57680 & 57692 & 57703 & $577^{15}$ & 57726 & $5773^{8}$ & 8 \\
\hline .78 & 57749 & 57761 & 57772 & $577^{84}$ & 57795 & $57 \mathrm{So} 7$ & 57818 & 57830 & $578_{4} 1$ & $57 \$_{52}$ & 10 \\
\hline .79 & 57864 & 57875 & 57887 & 57898 & 579 & 57921 & 57933 & 57944 & 57955 & 57967 & II \\
\hline 3.80 & $.57^{9} 97^{8}$ & $.579 \%$ & .58001 & $.5^{8013}$ & .58024 & 35 & .58047 & $.5^{\mathrm{S} 05^{8}}$ & $.5^{8}$ & .58 & 11 \\
\hline .81 & $5^{800,2}$ & 58104 & 58115 & 58127 & $5^{8} 1_{3}^{8}$ & $5^{8} 149$ & $5^{8} 161$ & $5^{8}$ & $5^{8}$ & $5^{8}$ & I \\
\hline .82 & $5^{8} 206$ & $5^{8218}$ & $5^{8229}$ & 58240 & $5^{8252}$ & $5^{8} 26_{3}$ & $5^{8} 274$ & 58286 & 58 & $5^{8} 300$ & 2 \\
\hline .83 & $5^{8} 320$ & $5^{8} 331$ & $5^{8} 343$ & $5^{8} 354$ & $5^{8} 3^{6} 5$ & $5^{8} 377$ & $5^{8} 3^{8 S}$ & $5^{8} .399$ & $5^{8}$ & $5^{5}+22$ & 3 \\
\hline .84 & $5^{8} 433$ & $5^{8} 444$ & $5^{8} 45^{6}$ & $5^{8} 467$ & $5^{8} 47^{8}$ & $5^{8} 490$ & $5^{8} 501$ & $5^{8} 512$ & $5^{8} 5^{24}$ & $5^{8} 535$ & 4 \\
\hline 3.85 & $.5^{8} 54^{6}$ & $.5^{8} 557$ & $.5^{8} 560$ & $.5^{8} 580$ & $.5^{8} 59^{1}$ & $.5^{8602}$ & .58614 & $.5^{8625}$ & $.5^{86} 63^{6}$ & .58647 & 6 \\
\hline & 58659 & $5^{8} 670$ & 58681 & $5^{8692}$ & $5^{87}$ & $5^{8}$ & $5^{8} 726$ & $5^{8} 737$ & $5^{8} 749$ & $5^{8} 760$ & 7 \\
\hline .87 & $5^{8} 771$ & $5^{8}-S_{2}$ & 58794 & $58 \mathrm{SO}_{5}$ & 58816 & 58.27 & $58 s_{3} \mathrm{~s}$ & $5^{8} \$ 50$ & $58 \$ 61$ & ${ }_{5} s_{72}$ & 8 \\
\hline .85 & $588_{3}$ & $5^{8} \sin 4$ & 58006 & 58917 & 58 y28 & $5^{8} 0.39$ & $5^{5} 95^{\circ}$ & 58061 & 58973 & $589 S_{4}$ & 9 \\
\hline .80 & $5^{8} 9095$ & 69006 & 59017 & 59028 & 5 & 59051 & 590612 & 59073 & 59084 & 59095 & 10 \\
\hline 3.90 & .59) 106 & .59118 & .59) 129 & .59140 & .59151 & .59162 & .59173 & .59184 & .59195 & .59207 & 11 \\
\hline .91 & 59218 & 59220 & 59240 & 59251 & 59262 & 59273 & 5) $2 \mathrm{~S}_{4}$ & 59205 & 59306 & 59318 & 1 \\
\hline .92 & 59329 & 59340 & 59351 & 59.362 & 59373 & $593^{84}$ & 59395 & 51) 406 & 59417 & 59428 & 2 \\
\hline .93 & 594.39 & 59450 & 59461 & 59472 & $5948_{3}$ & 59404 & 59500 & 50517 & 5952. & 59539 & 3 \\
\hline .94 & $5955^{\circ}$ & $595^{611}$ & 59572 & $595^{\mathrm{S}_{3}}$ & 59594 & 59605 & 59616 & 59627 & $5963 \mathrm{~s}$ & 59649 & 4 \\
\hline 3.95 & .59660 & .50671 & .50682 & $.59 \ln _{3}$ & .59704 & .59715 & .59726 & .50737 & .59748 & .59759 & 6 \\
\hline & 59770 & $59>50$ & 59791 & $59 \mathrm{Soz}$ & $59 \mathrm{Sr}_{3}$ & & $598_{35}$ & 59846 & $59 \mathrm{~S} 57$ & & 7 \\
\hline .97 & 50879 & 59800 & 59801 & 59912 & 59923 & 59934 & 59045 & 59956 & 59960 & & s \\
\hline .98 & 59988 & 59090 & 60010 & 10021 & 60032 & 6043 & 60054 & 60005 & 60 o 6 & 10086 & 9 \\
\hline .99 & 60097 & $60 \mathrm{ros}$ & $60114)$ & 60130 & (x) 141 & 60152 & 60163 & 60173 & $(10) 184$ & 60195 & 10 \\
\hline
\end{tabular}




\section{4. FIVE PLACE LOGARITHMS.}

LOGS. 5 PL.

\begin{tabular}{|c|c|c|c|c|c|c|c|c|c|c|c|}
\hline 0. & 0 & $\mathbf{I}$ & 2 & 3 & 4 & 5 & 6 & 7 & 8 & 9 & \\
\hline $\begin{array}{l}4.00 \\
.01 \\
.02 \\
.03 \\
.04\end{array}$ & $\begin{array}{l}.60206 \\
603^{14} \\
60423 \\
60531 \\
60638\end{array}$ & $\begin{array}{l}.60217 \\
60325 \\
60433 \\
60541 \\
60649\end{array}$ & $\begin{array}{l}60228 \\
60336 \\
60444 \\
6055^{2} \\
60660\end{array}$ & $\begin{array}{l}60239 \\
60347 \\
60455 \\
60563 \\
60670\end{array}$ & $\begin{array}{l}60249 \\
60358 \\
60466 \\
60574 \\
60681\end{array}$ & $\begin{array}{r}60260 \\
60369 \\
60477 \\
60584 \\
60692\end{array}$ & $\begin{array}{l}.60271 \\
60379 \\
60487 \\
60595 \\
60703\end{array}$ & $\begin{array}{l}60282 \\
60390 \\
60498 \\
60606 \\
60713\end{array}$ & $\begin{array}{l}60293 \\
60401 \\
60509 \\
60617 \\
60724\end{array}$ & $\begin{array}{l}60304 \\
60412 \\
60520 \\
60627 \\
60735\end{array}$ & $\begin{array}{r}11 \\
\text { I } \\
2 \\
3 \\
4\end{array}$ \\
\hline $\begin{array}{r}4.05 \\
.06 \\
.07 \\
.08 \\
.09\end{array}$ & $\begin{array}{l}60746 \\
60853 \\
60959 \\
61066 \\
61172\end{array}$ & $\begin{array}{l}60756 \\
60863 \\
60970 \\
61077 \\
61183\end{array}$ & $\begin{array}{l}60767 \\
60874 \\
60981 \\
61087 \\
61194\end{array}$ & $\begin{array}{l}60778 \\
60885 \\
60991 \\
61098 \\
61204\end{array}$ & $\begin{array}{ll}60 & 788 \\
60 & 895 \\
61 & 002 \\
61 & 109 \\
61 & 215\end{array}$ & $\begin{array}{r}60799 \\
60906 \\
61013 \\
61119 \\
61225\end{array}$ & $\begin{array}{l}.60810 \\
60917 \\
61023 \\
61130 \\
61236\end{array}$ & $\begin{array}{ll}60 & 821 \\
60 & 927 \\
61 & 034 \\
61 & 140 \\
61 & 247\end{array}$ & $\begin{array}{l}60831 \\
60938 \\
61045 \\
61151 \\
61257\end{array}$ & $\begin{array}{ll}60 & 842 \\
60 & 949 \\
61 & 055 \\
61 & 162 \\
61 & 268\end{array}$ & $\begin{array}{r}6 \\
7 \\
8 \\
9 \\
10\end{array}$ \\
\hline $\begin{array}{c}4.10 \\
.11 \\
.12 \\
.13 \\
.14\end{array}$ & $\begin{array}{ll}61 & 278 \\
61 & 384 \\
61 & 490 \\
61 & 595 \\
61 & 700\end{array}$ & $\begin{array}{ll}61 & 289 \\
61 & 395 \\
61 & 500 \\
61 & 606 \\
61 & 711\end{array}$ & $\begin{array}{cc}61 & 300 \\
61 & 405 \\
61 & 511 \\
61 & 616 \\
61 & 721\end{array}$ & $\begin{array}{l}61310 \\
61416 \\
61521 \\
61627 \\
61731\end{array}$ & $\begin{array}{l}613 \\
614 \\
615 \\
616 \\
617\end{array}$ & $\begin{array}{rr}6 & \text { I } 331 \\
61 & 437 \\
61 & 542 \\
61 & 648 \\
61 & 75^{2}\end{array}$ & $\begin{array}{l}61342 \\
61448 \\
61553 \\
6165^{8} \\
61763\end{array}$ & $\begin{array}{r}6135^{2} \\
6145^{8} \\
61563 \\
61669 \\
61773\end{array}$ & & & $\begin{array}{r}11 \\
1\end{array}$ \\
\hline $\begin{array}{r}4.15 \\
.16 \\
.17 \\
.18 \\
.19\end{array}$ & $\begin{array}{l}61805 \\
61909 \\
62014 \\
62118 \\
62221\end{array}$ & $\begin{array}{ll}61 & 815 \\
61 & 920 \\
62 & 024 \\
62 & 128 \\
62 & 232\end{array}$ & $\begin{array}{l}.61826 \\
61930 \\
62034 \\
62138 \\
62 \quad 242\end{array}$ & $\begin{array}{l}.61836 \\
61941 \\
62045 \\
62149 \\
62252\end{array}$ & & & $\begin{array}{l}.61868 \\
61972 \\
62076 \\
62180 \\
62284\end{array}$ & & & $\begin{array}{ll}61 & 899 \\
62 & 003 \\
62 & 107 \\
62 & 211 \\
62 & 315\end{array}$ & $\begin{array}{r}7 \\
8 \\
9 \\
10\end{array}$ \\
\hline $\begin{array}{c}4.20 \\
.21 \\
.22 \\
.23 \\
.24\end{array}$ & $\begin{array}{l}.62325 \\
62428 \\
62531 \\
62634 \\
62737\end{array}$ & $\begin{array}{l}.62 .335 \\
62439 \\
62542 \\
62644 \\
62747\end{array}$ & $\begin{array}{l}.62346 \\
62449 \\
62552 \\
62655 \\
62757\end{array}$ & $\begin{array}{l}.62356 \\
62459 \\
62562 \\
62665 \\
62767\end{array}$ & $\begin{array}{l}62469 \\
62572 \\
62675 \\
62778\end{array}$ & & & $\begin{array}{l}62500 \\
62603 \\
62706 \\
62808\end{array}$ & $\begin{array}{l}62408 \\
62511 \\
62613 \\
62716 \\
62818\end{array}$ & & $\begin{array}{r}10 \\
1 \\
2 \\
3 \\
4\end{array}$ \\
\hline $\begin{array}{r}4.25 \\
.26 \\
.27 \\
.28 \\
.29\end{array}$ & $\begin{array}{l}62839 \\
62941 \\
63043 \\
63144 \\
63246\end{array}$ & $\begin{array}{ll}62 & 849 \\
62 & 951 \\
63 & 053 \\
63 & 155 \\
63 & 256\end{array}$ & $\begin{array}{ll}.62 & 859 \\
62 & 961 \\
63 & 063 \\
63 & 165 \\
63 & 266\end{array}$ & $\begin{array}{l}62870 \\
62972 \\
63073 \\
63175 \\
63276\end{array}$ & $\begin{array}{l}.62880 \\
62982 \\
63083 \\
63185 \\
63286\end{array}$ & & $\begin{array}{l}.62900 \\
63002 \\
63104 \\
63205 \\
63306\end{array}$ & $\begin{array}{l}63012 \\
63114 \\
63215 \\
63317\end{array}$ & $\begin{array}{l}62921 \\
63022 \\
63124 \\
63225 \\
63327\end{array}$ & 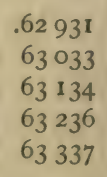 & $\begin{array}{l}5 \\
6 \\
7 \\
8 \\
9\end{array}$ \\
\hline $\begin{array}{c}4,30 \\
\cdot 31 \\
\cdot 32 \\
\cdot 33 \\
\cdot 34\end{array}$ & $\begin{array}{l}63347 \\
63448 \\
63548 \\
63649 \\
63749\end{array}$ & $\begin{array}{l}.63357 \\
63458 \\
6355^{8} \\
63659 \\
63759\end{array}$ & $\begin{array}{l}.63367 \\
63468 \\
63568 \\
63669 \\
63769\end{array}$ & $\begin{array}{l}63377 \\
63478 \\
63579 \\
63679 \\
63779\end{array}$ & & & & $\begin{array}{l}63417 \\
63518 \\
63619 \\
63719 \\
63819\end{array}$ & $\begin{array}{l}.63428 \\
63528 \\
63629 \\
63729 \\
63829\end{array}$ & & $\begin{array}{l}10 \\
1 \\
2 \\
3\end{array}$ \\
\hline $\begin{array}{r}4.35 \\
.36 \\
.37 \\
.38 \\
.39\end{array}$ & $\begin{array}{l}.63849 \\
63949 \\
64048 \\
64147 \\
64246\end{array}$ & $\begin{array}{l}.63859 \\
63959 \\
64058 \\
64157 \\
64256\end{array}$ & $\begin{array}{r}.63869 \\
63969 \\
64068 \\
64167 \\
64266\end{array}$ & $\begin{array}{r}.63879 \\
63979 \\
64078 \\
64177 \\
64276\end{array}$ & $\begin{array}{l}63889 \\
63988 \\
64088 \\
64187 \\
64286\end{array}$ & $\begin{array}{r}63899 \\
63998 \\
64098 \\
64197 \\
64296\end{array}$ & $\begin{array}{l}.63909 \\
64008 \\
64108 \\
64207 \\
64306\end{array}$ & $\begin{array}{r}63919 \\
64018 \\
64118 \\
64217 \\
64316\end{array}$ & $\begin{array}{r}.63929 \\
64028 \\
64128 \\
64227 \\
64326\end{array}$ & $\begin{array}{r}63939 \\
64038 \\
64137 \\
64237 \\
64335\end{array}$ & $\begin{array}{l}0 \\
7 \\
8 \\
9\end{array}$ \\
\hline $\begin{array}{c}4.40 \\
.41 \\
.42 \\
.43 \\
.44\end{array}$ & $\begin{array}{l}.64345 \\
64444 \\
64542 \\
64640 \\
6473^{8}\end{array}$ & $\begin{array}{l}.64355 \\
64454 \\
64552 \\
64650 \\
64748\end{array}$ & $\begin{array}{l}.64365 \\
64464 \\
64562 \\
64660 \\
6475^{8}\end{array}$ & $\begin{array}{l}64375 \\
64473 \\
64572 \\
64670 \\
64768\end{array}$ & $\begin{array}{l}.64385 \\
64483 \\
64582 \\
64680 \\
64777\end{array}$ & $\begin{array}{r}.64395 \\
64493 \\
64591 \\
64689 \\
64787\end{array}$ & $\begin{array}{l}.64404 \\
64503 \\
64601 \\
64699 \\
64797\end{array}$ & $\begin{array}{l}.64414 \\
64513 \\
64611 \\
64709 \\
64807\end{array}$ & $\begin{array}{r}.64424 \\
64523 \\
64621 \\
64719 \\
64816\end{array}$ & $\begin{array}{l}64434 \\
64532 \\
64631 \\
64729 \\
64826\end{array}$ & $\begin{array}{l}\text { I } \\
2 \\
3 \\
4\end{array}$ \\
\hline $\begin{array}{r}4.45 \\
.46 \\
.47 \\
.48 \\
.49\end{array}$ & $\begin{array}{ll}64 & 836 \\
64 & 933 \\
65 & 031 \\
65 & 128 \\
65 & 225\end{array}$ & $\begin{array}{ll}.64 & 846 \\
64 & 943 \\
65 & 040 \\
65 & 137 \\
65 & 234\end{array}$ & 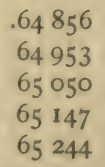 & $\begin{array}{ll}64 & 865 \\
64 & 963 \\
65 & 060 \\
65 & 157 \\
65 & 254\end{array}$ & $\begin{array}{l}.64875 \\
64972 \\
65070 \\
65167 \\
65263\end{array}$ & $\begin{array}{ll}64 & 885 \\
64 & 982 \\
65 & 079 \\
65 & 176 \\
65 & 273\end{array}$ & $\begin{array}{ll}.64 & 895 \\
64992 \\
65089 \\
65 \quad 186 \\
65283\end{array}$ & $\begin{array}{l}64904 \\
65002 \\
65099 \\
65196 \\
65292\end{array}$ & $\begin{array}{l}65 \text { ol } 1 \\
65108 \\
65205 \\
65302\end{array}$ & $\begin{array}{l}.64924 \\
65021 \\
65118 \\
65215 \\
65312\end{array}$ & $\begin{array}{l}5 \\
5 \\
6 \\
7 \\
8\end{array}$ \\
\hline
\end{tabular}


5 PL. LOGS.

\begin{tabular}{|c|c|c|c|c|c|c|c|c|c|c|c|}
\hline No. & 0 & I & 2 & 3 & 4 & 5 & 6 & 7 & 8 & 9 & $\begin{array}{l}\text { INTP } \\
\text { TAB. }\end{array}$ \\
\hline 4.50 & .65321 & .65331 & .65341 & .65350 & .65360 & .65369 & .65379 & .65389 & .65398 & .65408 & 10 \\
\hline .51 & 65418 & 65427 & 65437 & 65447 & 65456 & 65466 & 65475 & 65485 & 65495 & 65504 & I \\
\hline .52 & $655^{14}$ & 65523 & 65533 & 65543 & 65552 & 65562 & $6557^{1}$ & $655^{81}$ & $6559 \mathrm{I}$ & 65600 & 2 \\
\hline .53 & 65610 & 65619 & 65629 & 65639 & $6_{5} 648$ & 65658 & 65667 & 65677 & 65686 & 65696 & 3 \\
\hline .54 & 65706 & 65715 & 65725 & 65734 & 65744 & 65753 & 65763 & $6577^{2}$ & 65782 & 65792 & 4 \\
\hline $4 \cdot 55$ & $.65 \mathrm{Sor}$ & .65811 & .65820 & .65830 & .65839 & .65849 & $.6585^{8}$ & .65868 & .65877 & .65887 & 5 \\
\hline .56 & 65896 & 65906 & 65916 & 65925 & 65935 & 65944 & 65954 & 65963 & 65973 & 65982 & 6 \\
\hline .57 & 65992 & 66001 & 66011 & 66020 & $66 c$ & 66039 & 66049 & 66058 & & 66077 & 7 \\
\hline $.5^{8}$ & 66087 & 66096 & 66106 & 66115 & 66124 & 66134 & 66143 & 66153 & 66162 & 66172 & 8 \\
\hline .59 & 66181 & 66191 & 66200 & 66210 & 66219 & 66229 & $6623^{8}$ & 66247 & 66257 & 66266 & 9 \\
\hline 4.60 & .66276 & .66285 & .66295 & .66304 & .66314 & .66323 & .66332 & .66342 & $.6635 \mathrm{I}$ & $.6636 \mathrm{I}$ & 9 \\
\hline .61 & 66370 & $663^{80}$ & 66389 & 66398 & 66408 & 66417 & 66427 & 66436 & 66445 & 66455 & I \\
\hline .62 & 66464 & 66474 & $664^{8} 3$ & 66492 & 66502 & 66511 & 66521 & 66530 & 66539 & 66549 & 2 \\
\hline .63 & $6655^{8}$ & $665^{67}$ & 66577 & 66586 & 66596 & 66605 & 66614 & 66624 & 66633 & 66642 & 3 \\
\hline .64 & 66652 & 66661 & 66671 & 66680 & 66689 & 66699 & 66708 & 66717 & 66727 & 66736 & 4 \\
\hline 65 & .66745 & .66755 & .66764 & .66773 & .66783 & .66792 & .66801 & .66811 & .66820 & .06829 & 5 \\
\hline 66 & 66839 & 66848 & 66857 & 66867 & 66876 & 66885 & 66894 & 66904 & 66913 & 66922 & 5 \\
\hline .67 & 66932 & 66941 & 66950 & 66960 & 66969 & $6697^{8}$ & 66987 & 66997 & 67006 & 67015 & 6 \\
\hline .68 & 67025 & 67034 & 67043 & 67052 & 67062 & 67071 & 67080 & 67089 & 67090 & 67108 & 7 \\
\hline .69 & 67117 & 67127 & 67136 & 67145 & 67 I54 & 67164 & 67173 & 67182 & 67191 & 67201 & 8 \\
\hline 4.70 & .67210 & .67219 & .67228 & .67237 & .67247 & .67256 & .67265 & .67274 & .67284 & .67 & 9 \\
\hline .71 & 67302 & 67311 & 67321 & 67330 & 67339 & 67348 & 67357 & 67367 & 67376 & $673^{85}$ & I \\
\hline .72 & 67394 & 67403 & $674^{1} 3$ & 67422 & 67431 & 67440 & 67449 & 67459 & 67468 & 67477 & 2 \\
\hline .73 & 67486 & 67495 & 67504 & 67514 & $675^{23}$ & 67532 & 67541 & 67550 & 67560 & 67569 & 3 \\
\hline$\cdot 74$ & $6757^{8}$ & $675^{87}$ & 67596 & 67605 & 67614 & 67624 & 67633 & 67642 & 67651 & 67660 & 4 \\
\hline 4.75 & .67669 & .67679 & .67688 & .67697 & .67706 & .67715 & .67724 & .67733 & .67742 & .67752 & 5 \\
\hline & 67761 & 67770 & 67779 & 67788 & 67797 & 67806 & 67815 & 67825 & 67834 & 43 & 5 \\
\hline 77 & 67852 & $6786 \mathrm{I}$ & 67870 & 67879 & 67888 & 67897 & 67906 & 67916 & 67925 & 67934 & 6 \\
\hline$-7^{8}$ & 67943 & 67952 & 67961 & 67970 & 67979 & 67988 & 67997 & 68006 & 68 o1 5 & 68024 & 7 \\
\hline .79 & $68 \circ 34$ & 68043 & 68052 & $6806 \mathrm{I}$ & 68070 & 9 & 68088 & 7 & 68106 & 68115 & 8 \\
\hline 4.80 & .68124 & $.68 \mathrm{I} 33$ & .68142 & .68 I 51 & .68160 & .68169 & $.6817^{8}$ & .68187 & .68196 & .68 & 9 \\
\hline .81 & 68215 & 68224 & 68233 & 68242 & 68251 & 68260 & 68269 & 68278 & 68257 & 68296 & 1 \\
\hline .82 & 68305 & $683^{14}$ & 68323 & 68332 & $6834^{1}$ & 68350 & 68359 & 68368 & 68377 & $683^{86}$ & 2 \\
\hline .83 & 68395 & 68404 & $68+13$ & 68422 & 68431 & 68440 & 68449 & $6845^{8}$ & $684^{67}$ & 68476 & 3 \\
\hline .84 & 68485 & 68494 & 68502 & 68511 & 68520 & 68529 & 68538 & 68547 & 68556 & 68565 & 4 \\
\hline 4.85 & .68574 & $.685^{8} 3$ & .68592 & $.6860 \mathrm{I}$ & .68610 & .68619 & .68628 & .68637 & .68646 & .68655 & 5 \\
\hline .86 & 68664 & 68673 & $6868 \mathrm{I}$ & 68690 & 68699 & 68708 & 68717 & 68726 & 68735 & 68744 & 5 \\
\hline .87 & 68753 & 68762 & 68771 & 68780 & 68789 & 68797 & 68806 & 68815 & 68824 & $688_{33}$ & 6 \\
\hline .88 & $688_{42}$ & 68851 & 68860 & 68869 & 68878 & 68586 & 68805 & 68904 & 68913 & 65922 & 7 \\
\hline .80 & 68931 & 68940 & 68949 & $6895^{8}$ & 68966 & 68975 & 68984 & 68993 & 69002 & 6011 & 8 \\
\hline 4.90 & 69020 & .6028 & .69037 & .69046 & .69055 & 64 & 73 & 82 & 90 & & 8 \\
\hline .91 & (x) 108 & 60117 & 69126 & 69135 & 69144 & 69152 & 69161 & $6 x+170$ & 69179 & 69 ISs & 1 \\
\hline .92 & 6x) 197 & 69205 & 69214 & 69223 & 69232 & 60) 241 & $(x) 249$ & (x) 258 & 692677 & (x) 276 & 2 \\
\hline .93 & (6) 285 & (x) 294 & 60,302 & (x) 311 & (6) $3^{20}$ & 60329 & $60,33^{8}$ & (x) $34^{6}$ & (x) 355 & 6) 364 & 2 \\
\hline .94 & 69373 & $693^{81}$ & 69390 & 69 399 & 69408 & 69417 & 69425 & $6 x+34$ & 69443 & 6) 452 & 3 \\
\hline 4.95 & .60461 & .69469 & . $6097^{8}$. & 60487 & 69496 & (x) 504 & (x) 513 & .6x) 522 & (x) 531 & (x) 539 & 4 \\
\hline .96 & 69548 & 69557 & 69566 & 69574 & $695^{8} 3$ & 6) 592 & 6) 601 & $60(609$ & 69618 & (x) 627 & 5 \\
\hline .97 & 69636 & 69644 & 69653 & 69662 & 69671 & 609679 & (6) GS8 & $6 x) 607$ & 69705 & (x) 714 & 6 \\
\hline .98 & $697^{23}$ & 69732 & 69740 & 69749 & $6975^{8}$ & 69767 & (x) 775 & (x) 784 & (x) 703 & $(x) .501$ & 6 \\
\hline .99 & $6 x 810$ & 69819 & 69827 & 69836 & 69845 & 69854 & (x) $\mathrm{S} 62$ & $(x) 871$ & 60.850 & (x) 588 & 7 \\
\hline
\end{tabular}


LOGS. 5 PL.

\begin{tabular}{|c|c|c|c|c|c|c|c|c|c|c|c|}
\hline No. & 0 & & 2 & 3 & 4 & 5 & 6 & 7 & 8 & 9 & $\begin{array}{l}\text { INTP } \\
\text { TAB. }\end{array}$ \\
\hline 5.00 & $.69 S_{97}$ & .69906 & .69914 & .69923 & .69932 & .69940 & .69949 & $.6995^{8}$ & .69966 & .69975 & 9 \\
\hline .01 & $699 S_{4}$ & 69992 & 70001 & 70010 & 70018 & 70027 & 70036 & 70044 & 70053 & 70062 & I \\
\hline .02 & 70070 & 70079 & 70088 & 70096 & 70105 & 70114 & 70122 & 70131 & 70140 & 70148 & 2 \\
\hline .03 & 70157 & 70165 & 70174 & $7018_{3}$ & 70191 & 70200 & 70209 & 70217 & 70226 & 70234 & 3 \\
\hline .04 & 70243 & 70252 & 70260 & 70269 & 70278 & 70286 & 70295 & 70303 & 70312 & 70321 & 4 \\
\hline 5.05 & .70329 & $.7033^{8}$ & .70346 & .70355 & .70364 & .70372 & $.7038 \mathrm{I}$ & .70389 & .70398 & .70406 & 5 \\
\hline .06 & 70415 & 70424 & 70432 & $7044 I$ & 70449 & $7045^{8}$ & 70467 & 70475 & 70484 & 70492 & 5 \\
\hline .07 & 70501 & 70509 & 70518 & 70526 & 70535 & 70544 & $7055^{2}$ & $7056 r$ & 70569 & 70578 & 6 \\
\hline .08 & 70586 & 70595 & 70603 & 70612 & 70621 & 70629 & 70638 & 70646 & 70655 & 70663 & 7 \\
\hline .09 & 70672 & 70680 & 70689 & 70697 & 70706 & 70714 & 70723 & 70731 & 70740 & 70749 & 8 \\
\hline 5.10 & .70757 & .70766 & & 783 & .70791 & .70800 & .70808 & .70817 & .70825 & .70834 & 8 \\
\hline .II & 70842 & 70851 & 70859 & 70868 & 70876 & 70885 & 70893 & 70902 & 70910 & 70919 & I \\
\hline .12 & 70927 & 70935 & 70944 & 70952 & 7096 I & 70969 & 70978 & 70986 & 70995 & 71003 & 2 \\
\hline .13 & 71012 & 71020 & 71029 & 71037 & 71046 & 71054 & . 71063 & 71071 & 71079 & 71088 & 2 \\
\hline .14 & 71096 & 71105 & 71113 & 71122 & 71130 & 71139 & 71147 & 71155 & 71164 & 71172 & 3 \\
\hline 5.15 & .71 I 81 & .71189 & .71198 & .71206 & .71214 & .71223 & .71231 & .71240 & .71248 & .71257 & 4 \\
\hline .16 & 71265 & 71273 & 71282 & 71290 & 71299 & 71307 & 71315 & 71324 & 71332 & 71341 & 5 \\
\hline .17 & 7 I 349 & 71357 & 71366 & 7I 374 & 71383 & 71391 & 71399 & 71408 & 71416 & 71425 & 6 \\
\hline .18 & 71433 & $7144 I$ & 71450 & $7145^{8}$ & 71466 & 71475 & 71483 & 71492 & 71500 & 71508 & 6 \\
\hline .19 & 71517 & 71525 & 71533 & 71542 & $7155^{\circ}$ & 71559 & 71567 & 71575 & 71584 & 71592 & 7 \\
\hline 5.20 & .71600 & .71609 & .71617 & .71625 & .71634 & .71642 & .71650 & .71659 & & .71675 & 8 \\
\hline .21 & 71684 & 71692 & 71700 & 71709 & 71717 & 71725 & 71734 & 71742 & $7175^{\circ}$ & 71759 & I \\
\hline .22 & 71767 & 71775 & 71784 & 71792 & 71800 & 71809 & 71817 & 71825 & 71834 & 71842 & 2 \\
\hline .23 & 71850 & 71858 & 71867 & 71875 & 71883 & 71892 & 71900 & 71908 & 71917 & 71925 & 2 \\
\hline .24 & 71933 & 7 I 941 & $7195^{\circ}$ & $7195^{8}$ & 71966 & 71975 & 71983 & 71991 & 71999 & 72008 & 3 \\
\hline 5.25 & .72016 & .72024 & .72032 & .72041 & .72049 & .72057 & .72066 & .72074 & .72082 & .72090 & 4 \\
\hline .26 & 72099 & 72107 & 72115 & 72123 & 72132 & 72140 & 72148 & 72 I 56 & 72165 & 72173 & .5 \\
\hline .27 & 72181 & 72189 & 72198 & 72206 & 72214 & 72222 & 72230 & 72239 & 72247 & 72255 & 6. \\
\hline .28 & 72263 & 72272 & 72280 & 72288 & 72296 & 72304 & $7^{2} 313$ & 72321 & 72329 & 72337 & 6 \\
\hline .29 & $7234^{6}$ & 72354 & 72362 & 72370 & $7237^{8}$ & $7^{2} 3^{87}$ & 72395 & 72403 & 72411 & $724 \times 9$ & 7 \\
\hline 5.30 & .72428 & .72436 & .72444 & $.7245^{2}$ & .72460 & .72469 & .72477 & .72485 & .72493 & .72 & 8 \\
\hline $.3 I$ & 72509 & 72518 & 72526 & 72534 & 72542 & $7255^{\circ}$ & $7255^{8}$ & 72567 & 72575 & $7^{2} 5^{8} 3$ & I \\
\hline .32 & 72591 & 72599 & 72607 & 72616 & 72624 & 72632 & 72640 & 72648 & 72656 & 72665 & 2 \\
\hline .33 & 72673 & $7268 \mathrm{I}$ & 72689 & 72697 & 72705 & 72713 & 72722 & 72730 & $7273^{8}$ & 72746 & 2 \\
\hline .34 & 72754 & 72762 & 72770 & 72779 & 72787 & 72795 & 72803 & $7281 \mathrm{I}$ & 72819 & 72827 & 3 \\
\hline $5 \cdot 35$ & .72835 & .72843 & .72852 & .72860 & .72868 & .72876 & .72884 & .72892 & .72900 & .72908 & 4 \\
\hline .36 & 72916 & 72925 & 72933 & 72941 & 72949 & 72957 & 72965 & 72973 & $7298 \mathrm{I}$ & 72989 & 5 \\
\hline$\cdot 37$ & 72997 & 73006 & 73 Or 4 & 73022 & 73030 & $7303^{8}$ & 73046 & 73054 & 73062 & 73070 & 6 \\
\hline .38 & 73078 & 73086 & 73094 & 73102 & 73 I I I & 73119 & 731 & 73 & 73 & $7315 \mathrm{I}$ & 6 \\
\hline .39 & 73159 & 73167 & 73175 & 73 I83 & 73 I9I & 73199 & 73207 & 73215 & 73223 & 73231 & 7 \\
\hline 5.40 & .73239 & .73247 & .73255 & .73263 & .73272 & .73280 & .73288 & .73296 & & .73312 & 7 \\
\hline .41 & 73320 & 73328 & 73336 & 73344 & $7335^{2}$ & 73360 & 73368 & $7337^{6}$ & 73384 & 73392 & I \\
\hline .42 & 73400 & 73408 & 73416 & 73424 & $7343^{2}$ & 73440 & $7344^{8}$ & $7345^{6}$ & 73464 & 73472 & I \\
\hline .43 & 73480 & 73488 & 73496 & 73504 & 73512 & 73520 & 73528 & 73536 & 73544 & $7355^{2}$ & 2 \\
\hline .44 & 73560 & 73568 & 73576 & 73584 & 73592 & 73600 & 73608 & 73616 & 73624 & 73632 & 3 \\
\hline 5.45 & .73640 & $.7364^{8}$ & .73656 & .73664 & .73672 & .73679 & .73687 & .73695 & .73703 & .73711 & 4 \\
\hline .46 & 73719. & .73727 & 73735 & 73743 & $7375^{1}$ & 73759 & 73767 & 73775 & $737^{8} 3$ & 73791 & 4 \\
\hline .47 & 73799 & 73807 & 73815 & 73823 & 73830 & 73838 & 73846 & 73854 & 73862 & 73870 & 5 \\
\hline .48 & 73878 & 73886 & 73894 & 73902 & 73910 & 73918 & 73926 & 73933 & 73941 & 73949 & 6 \\
\hline .49 & 73957 & 73965 & 73973 & $7398 \mathrm{I}$ & 73989 & 73997 & 74005 & 74 OI 3 & 74020 & 74028 & 6 \\
\hline
\end{tabular}

LOGS. 5 PL.

5.

(20) 


\begin{tabular}{|c|c|c|c|c|c|c|c|c|c|c|c|}
\hline No. & 0 & I & 2 & 3 & 4 & 5 & 6 & 7 & 8 & 9 & $\begin{array}{l}\text { INTP } \\
\text { TAB. }\end{array}$ \\
\hline 5.50 & .74036 & .740 .44 & .74052 & .74060 & .74068 & .74076 & $.7408_{4}$ & .74092 & .74099 & .74107 & 8 \\
\hline .51 & 74115 & $74 \quad 123$ & 74131 & 74139 & 74147 & 74155 & 74162 & 74170 & 74178 & 74186 & I \\
\hline .52 & 74194 & 74202 & 74210 & 74218 & 74225 & 74233 & 74241 & 74249 & 74257 & 74265 & 2 \\
\hline .53 & 74273 & 74280 & $7+288$ & $7+296$ & 74304 & 74312 & 74320 & 74327 & 74335 & 74343 & 2 \\
\hline .54 & 74351 & 74359 & 74367 & 74374 & $7+3 S_{2}$ & 74390 & 74398 & 74406 & $744^{14}$ & 74421 & 3 \\
\hline 5.55 & .74429 & .74437 & .74445 & .74453 & .74461 & .74468 & .74476 & .74484 & .74492 & .74500 & 4 \\
\hline .56 & 74507 & 74515 & $745^{23}$ & 74531 & 74539 & 74547 & 74554 & $7+562$ & 74570 & 74578 & 5 \\
\hline .57 & $745 \$ 6$ & 74593 & 74601 & 74609 & 74617 & $74^{624}$ & 74632 & 74640 & 74648 & 74656 & 6 \\
\hline .58 & 74663 & 74671 & 74679 & 74687 & 74695 & 74702 & 74710 & 74718 & 74726 & 74733 & 6 \\
\hline .59 & 74741 & 74749 & 74757 & 74764 & $7477^{2}$ & 74750 & 74788 & 74796 & $74 \mathrm{SO}_{3}$ & 74811 & 7 \\
\hline 5.60 & .74819 & .74827 & $.74 \delta_{34}$ & .74842 & .74850 & .74858 & .74865 & .74873 & $.7488 \mathrm{I}$ & .74889 & 7 \\
\hline $.6 \mathrm{I}$ & 74896 & 74904 & 74912 & 74920 & 74927 & 74935 & 74943 & $7495^{\circ}$ & $7+95^{8}$ & 74966 & 1 \\
\hline .62 & 74974 & 74981 & 74989 & 74997 & 75005 & 75012 & 75020 & 75028 & $75 \bigcirc 35$ & 75043 & I \\
\hline .63 & 75051 & 75059 & 75066 & 75074 & 75082 & 75089 & 75097 & 75105 & 75113 & 75120 & 2 \\
\hline .64 & 75128 & 75136 & 75143 & 75151 & 75159 & 75166 & 75174 & 75182 & 75189 & 75197 & 3 \\
\hline 5.65 & .75205 & .75213 & .75220 & .75228 & .75236 & .75243 & .75251 & .75259 & .75266 & .75274 & 4 \\
\hline .66 & 75282 & $75^{289}$ & 75297 & 75305 & 75312 & 75320 & 75328 & 75335 & 75343 & 75351 & 4 \\
\hline .67 & $7535^{8}$ & 75366 & 75374 & 75381 & 75389 & 75397 & 75404 & 75412 & 75420 & 75427 & 5 \\
\hline .68 & 75435 & 75442 & 75450 & $7545^{8}$ & 75465 & 75473 & $7548 I$ & 75488 & 75496 & 75504 & 6 \\
\hline .69 & $755^{11}$ & 75519 & $755^{26}$ & 75534 & 75542 & 75549 & 75557 & 75565 & $7557^{2}$ & 75580 & 6 \\
\hline 5.70 & $.755^{87}$ & & .75 & 610 & .75618 & .75626 & .75633 & .75641 & .75648 & .75656 & 8 \\
\hline .71 & 75664 & 75671 & 75679 & 75686 & 75694 & 75702 & 75709 & 75717 & 75724 & 75732 & I \\
\hline .72 & 75740 & 75747 & 75755 & 75762 & 75770 & $7577^{8}$ & 75785 & 75793 & 75800 & 75808 & 2 \\
\hline .73 & 75815 & 75823 & 75831 & $75^{838}$ & 75846 & 75853 & 75861 & 75868 & 75876 & 75884 & 2 \\
\hline .74 & $75^{891}$ & $75^{899}$ & 75906 & 75914 & 75921 & 75929 & 75937 & 75944 & $7595^{2}$ & 75959 & 3 \\
\hline 5.75 & .75967 & .75974 & .75982 . & .75989 & .75 & .76005 & .76012 & .76020 & .76027 & .76035 & 4 \\
\hline .75 & 76042 & 76050 & 76057 & 76065 & 76072 & 76080 & 76087 & 76095 & 76103 & 10 & 5 \\
\hline .77 & 76118 & 76125 & $7^{6} \quad 133$ & 76140 & 76148 & 76155 & 76163 & 76170 & 76178 & 76 is 5 & 6 \\
\hline $.7^{8}$ & 76193 & 76200 & 76208 & 76215 & 76223 & 76230 & 76238 & 76245 & 76253 & 76260 & 6 \\
\hline .79 & 76268 & 76275 & 76283 & 76290 & 76298 & 76305 & $7^{6} 313$ & $7^{6} 320$ & 76328 & 76335 & 7 \\
\hline 5.80 & .76343 & .76350 & $.7^{6} 35^{8}$ & $.7^{6} 365$ & .76373 & .76380 & .76388 & .76395 & .76403 & .76410 & 7 \\
\hline 81 & 76418 & 76425 & 76433 & 76440 & 76448 & 76455 & 76462 & 76470 & $7^{6}+77$ & $764^{8} 5$ & 1 \\
\hline .82 & $7^{6} 49^{2}$ & 76500 & 76507 & 76515 & 76522 & 76530 & 76537 & 76545 & 76552 & 76559 & 1 \\
\hline .83 & $7^{6} 567$ & $7^{6} 574$ & 76582 & 76589 & 76597 & 76604 & 76612 & 76619 & 76626 & 76634 & 2 \\
\hline .84 & $7^{66} 641$ & 76649 & 76656 & 76664 & 76671 & 76678 & 76686 & 76693 & 76701 & $7^{6} 708$ & 3 \\
\hline 5.85 & .76716 & .76723 & .76730 & .76738 & .76745 & .76753 & .76760 & .76768 & .76775 & $.767^{32}$ & 4 \\
\hline .86 & 76790 & $7^{6} 797$ & $76 \mathrm{SO}_{5}$ & 76812 & 76819 & 76827 & 76834 & $76 s_{42}$ & $-6 S_{49}$ & 76856 & 4 \\
\hline .87 & 76864 & 76871 & 76879 & 76886 & 76893 & 76901 & 76908 & 76916 & 76923 & 760,30 & 5 \\
\hline $.8 \mathrm{~s}$ & 76935 & 76945 & 76953 & 76960 & 76967 & 76975 & 76982 & 76989 & 76997 & 77004 & 6 \\
\hline .89 & 77012 & 77019 & 77026 & 77034 & 77041 & 77048 & 77056 & 77063 & 77070 & 77075 & 6 \\
\hline 5.90 & $.7708_{5}$ & .77093 & .77100. & .77107 & & .77122 & 29 & & .77144 & 51 & 7 \\
\hline .91 & 77159 & 77166 & 77173 & 77181 & 77188 & 77195 & 77203 & 77210 & 77217 & 77225 & 1 \\
\hline .92 & 77232 & 77240 & 77247 & 77254 & 77262 & 77269 & 77276 & 77283 & 77291 & 77205 & 1 \\
\hline .93 & 77305 & 77313 & 77320 & 77327 & 77335 & $7734^{2}$ & 77349 & 77357 & 77364 & 77371 & 2 \\
\hline .24 & 77379 & $773^{86}$ & 77.393 & 77401 & 77408 & 77415 & $77+22$ & 77430 & 77437 & 77444 & 3 \\
\hline 5.95 & .77452 & .77459 & .77466 & .77474 & $.77+81$ & .77488 & .77495 & .77503 & 77510 & .77517 & 4 \\
\hline .00 & 77525 & 775.32 & 77539 & $77.54^{6}$ & 77554 & $775^{618}$ & 77565 & $7757^{6}$ & $775^{8} 3$ & 77590 & 4 \\
\hline .97 & 77597 & 77005 & 77612 & 77619 & 77627 & 77634 & 77 C.41 & 77648 & 77656 & 77603 & 5 \\
\hline 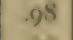 & 77670 & 77677 & $7765_{5}$ & $77 \log 2$ & 77609 & 77706 & $77 \div 14$ & 77721 & $777^{25}$ & 77735 & 6 \\
\hline .99 & 77743 & 77750 & 77757 & 7776.4 & 77772 & 77779 & 77786 & 77793 & 77 Sol & $77 \mathrm{sos}$ & 6 \\
\hline
\end{tabular}


LOGS. 5 PL.

\begin{tabular}{|c|ccccc}
\hline No: & 0 & I & 2 & 3 & 4 \\
\hline 6.00 & .77815 & .77822 & .77830 & .77837 & .77844 \\
.01 & 77887 & 77895 & 77902 & 77909 & 77916 \\
.02 & 77960 & 77967 & 77974 & 77981 & 77988 \\
.03 & 78032 & 78039 & 78046 & 78053 & 78061 \\
.04 & 78104 & 78111 & 78118 & 78125 & 78132 \\
6.05 & .78176 & 78183 & .78190 & .78197 & .78204 \\
.06 & 78247 & 78254 & 78262 & 78269 & 78276 \\
.07 & 78319 & 78326 & 78333 & 78340 & 78347 \\
.08 & 78390 & 78398 & 78405 & 78412 & 78419 \\
.09 & 78462 & 78469 & 78476 & 78483 & 78490 \\
\hline
\end{tabular}

6.10

\section{.II}

.12

.13

.14

6.15

.16

.17

.18

.I9

6.20

.21

.23

.24

6.25

.26

.27

.28

.29

6.30

.3I

.32

.33

.34

6.35

.36

.37

.38

.39

6.40

.41

.42
.43

.44

6.45

$. 7 7 \longdiv { 4 6 }$

$.84 \quad .47$

.48
.49
.78533 .78540 .78547 .78554 .78561 $\begin{array}{lllllll}78604 & 78611 & 78618 & 78625 & 78633\end{array}$ $\begin{array}{lllllll}78675 & 78682 & 78689 & 78696 & 78 & 704\end{array}$

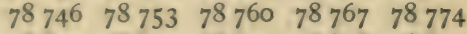
$\begin{array}{lllllll}78817 & 78824 & 78831 & 78838 & 78845\end{array}$ $.78888 .78895 \cdot 78902.78909 .78916$ $\begin{array}{llllllll}78958 & 78 & 965 & 78972 & 78979 & 78986\end{array}$ $\begin{array}{lllllllll}79029 & 79036 & 79043 & 79050 & 79057\end{array}$ $\begin{array}{lllllllll}79 & 099 & 79 & 106 & 79 & 113 & 79 & 120 & 79127\end{array}$ $\begin{array}{lllllllll}79 & 169 & 79 & 176 & 79 & 183 & 79 & 190 & 79197\end{array}$

$\begin{array}{lllllll}.79 & 239.79246 .79253 .79 & 260 & .79 & 267\end{array}$ $\begin{array}{llllllllll}79 & 309 & 79 & 316 & 79 & 323 & 79 & 330 & 79 & 337\end{array}$ $\begin{array}{llllllllll}79 & 379 & 79 & 386 & 79 & 393 & 79 & 400 & 79 & 407\end{array}$ $\begin{array}{llllllll}79449 & 79456 & 79463 & 79470 & 79477\end{array}$ $\begin{array}{lllllll}79 & 518 & 79 & 525 & 79532 & 79539 & 79546\end{array}$ .79588 .79595 .79602 .79609 .79616 $\begin{array}{lllllllll}79657 & 79664 & 79 & 671 & 79 & 678 & 79685\end{array}$

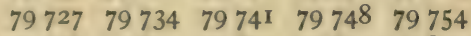
$\begin{array}{lllllllll}79796 & 79803 & 79810 & 79817 & 79824\end{array}$ $\begin{array}{lllllll}79865 & 79872 & 79879 & 79886 & 79893\end{array}$

$.79934 .7994 I \quad 79948.79955 .79962$ 8000380010800178002480030 $\begin{array}{lllllll}80072 & 80079 & 80085 & 80092 & 80099\end{array}$ 80 I $40 \quad 80$ I 4780 I54 80 I6I 80 I 68 $8020980216 \quad 802238022980236$

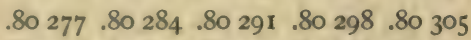
$80346 \quad 80353 \quad 80359 \quad 80366 \quad 80373$ $80414 \quad 80421 \quad 80428 \quad 804348044 I$ $80482 \quad 8048980496 \quad 80502 \quad 80509$ $80550 \quad 80557$ 80 564 80 $570 \quad 80577$

$\begin{array}{lllllll}.80618 & .80625 & .80632 & .80638 & .80645\end{array}$ $8068680693 \quad 8069980706 \quad 80713$ $80754 \quad 80760 \quad 80767 \quad 80774 \quad 8078 \mathrm{I}$ $\begin{array}{lllllll}80821 \quad 80828 & 80835 \quad 80841 \quad 80848\end{array}$ 8088980895809028090980916 $\begin{array}{llllll}.80956 & .80963 & .80969 & .80976 \quad .80983\end{array}$ $\begin{array}{llllllllllllllllll}81023 & 81030 & 81037 & 81043 & 81050\end{array}$ $\begin{array}{llllllllllllllll}81090 & 81097 & 81 & 104 & 81 & \text { III } & 81 & 117\end{array}$ $\begin{array}{llllllllllll}81 & 158 & 81 & 164 & 81 & 17 I & 81 & 178 & 81 & 184\end{array}$ $\begin{array}{llllllllllll}81 & 224 & 81 & 23 I & 8 I & 238 & 81 & 245 & 81 & 25 I\end{array}$ $\begin{array}{lllllll}.77851 & .77859 & .77866 & .77873 & .77880\end{array}$ $\begin{array}{lllllll}77924 & 77931 & 77938 & 77945 & 77952\end{array}$ $\begin{array}{llllllll}77996 & 78003 & 78010 & 78017 & 78025\end{array}$ $\begin{array}{lllllll}78068 & 78075 & 78082 & 78089 & 78097\end{array}$ $\begin{array}{lllllllll}78 & 140 & 78 & 147 & 78 & 154 & 78 & 161 & 78 \\ 168\end{array}$ $\begin{array}{lllllll}.78211 & .78219 & .78226 & .78233 & .78240\end{array}$ $\begin{array}{lllllllllll}78 & 283 & 78 & 290 & 78 & 297 & 78 & 305 & 78 & 312\end{array}$ $\begin{array}{lllllllllll}78 & 355 & 78 & 362 & 78 & 369 & 78 & 376 & 78 & 383\end{array}$

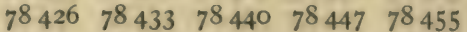

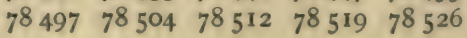

.78569 .78576 .78583 .78590 .78597 $\begin{array}{lllllllll}78640 & 78647 & 78654 & 78661 & 78668\end{array}$

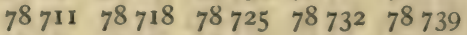
$\begin{array}{llllllll}7^{8} & 781 & 78 & 789 & 78 & 796 & 78803 & 78810\end{array}$ $\begin{array}{lllllll}78852 & 78859 & 78866 & 78873 & 78880\end{array}$ .78923 .78930 .78937 .78944 .7895 I $\begin{array}{lllllllll}78993 & 79000 & 79007 & 790 I 4 & 7902 I\end{array}$ $\begin{array}{llllllll}79064 & 79071 & 79078 & 79085 & 79092\end{array}$

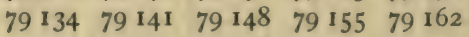
$\begin{array}{llllllll}79 & 204 & 79211 & 79218 & 79225 & 79232\end{array}$

$.79274 .79281 \quad .79288 .79295 \cdot 79302$ $\begin{array}{llllllllll}79 & 344 & 79 & 351 & 79 & 35^{8} & 79 & 365 & 79 & 372\end{array}$ $\begin{array}{lllllll}79414 & 79421 & 79428 & 79435 & 79442\end{array}$ $\begin{array}{llllllll}79484 & 79491 & 79498 & 79 & 505 & 79 & 5^{1} \text { I }\end{array}$

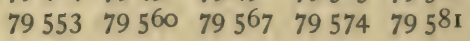
.79623 .79630 .79637 .79644 .79650 $\begin{array}{lllllllll}79 & 692 & 79699 & 79 & 706 & 79 & 713 & 79 & 720\end{array}$

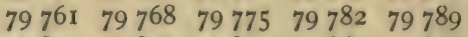
$\begin{array}{llllllll}79 & 831 & 79 & 837 & 79844 & 79851 & 79858\end{array}$ $\begin{array}{lllllll}79900 & 79906 & 79913 & 79920 & 79927\end{array}$

.79969 .79975 .79982 .79989 .79996 $800_{37} 800_{44} 800_{5}$ I $800_{5} 880065$ $\begin{array}{llllll}80 & 106 \quad 80 \text { II } 380 \text { I20 } & 80 \text { I27 } & 80 \text { I } 34\end{array}$ $\begin{array}{llllll}80 I 75 & 80182 & 80188 & 80195 \quad 80202\end{array}$ $8024380250 \quad 80257 \quad 802648027$ I

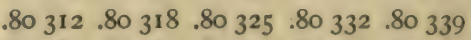
$\begin{array}{llllllllllllll}80 & 380 & 80 & 387 & 80 & 393 & 80 & 400 & 80 & 407\end{array}$ 80448 80 $455 \quad 80462 \quad 80468 \quad 80475$ 80516 80 523 80 530 80 $536 \quad 80543$ $805^{84} 8059$ I $80598 \quad 80604806$ I I

.80652 .80 659 $.80665 \quad .80672 \quad .80679$ $80720 \quad 80726 \quad 80733 \quad 80740 \quad 80747$ 80787 80 794 80 801 $80808 \quad 80814$ $\begin{array}{lllllll}80855 & 80862 & 80868 & 80875 & 80882\end{array}$ $809228092980936 \quad 8094380949$ .80990 .80996 .81003 .8 I 010.81 or 7

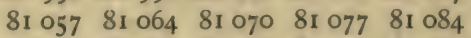
$\begin{array}{llllllllllllll}81 & 124 & 81 & \text { I3I } & 81 & \text { I } 37 & 8 \text { I I } 44 & 8 \text { I I5I }\end{array}$ $\begin{array}{llllllllllllll}81 & 19 I & 81 & 198 & 81 & 204 & 81 & 2 I I & 81 & 2 I\end{array}$ $\begin{array}{lllllllllll}81 & 258 & 81 & 265 & 81 & 271 & 81 & 278 & 81 & 285\end{array}$ 


\begin{tabular}{|c|c|c|c|c|c|c|c|c|c|c|c|}
\hline No. & 0 & I & 2 & 3 & 4 & 5 & 6 & 7 & 8 & 9 & $\begin{array}{l}\text { INTP. } \\
\text { TAB. }\end{array}$ \\
\hline 6.50 & .81291 & .81298 & .81305 & .81311 & .81318 & .81325 & .81331 & & .81345 & .81351 & 6 \\
\hline $.5^{1}$ & $8135^{\circ}$ & 81365 & 81371 & 81378 & $\mathrm{~S}_{1} \mathrm{~S}_{5}$ & 81391 & 81398 & 81405 & $81+11$ & 81418 & I \\
\hline .52 & 81425 & 81431 & $8143^{8}$ & S1 445 & 81451 & $8145^{8}$ & 81465 & 81471 & 81478 & 81485 & I \\
\hline .53 & 81491 & $8149^{8}$ & 81505 & 81511 & 81518 & 81525 & 81 531 & $8153^{8}$ & 81544 & 81551 & 2 \\
\hline .54 & $8155^{8}$ & 81564 & 81571 & $8157^{8}$ & 81584 & 81591 & 81598 & 81604 & 81611 & 81617 & 2 \\
\hline 6.55 & .81624 & .81631 & .81637 & .81644 & .81651 & .81657 & .81664 & .81671 & .81677 & .81684 & 3 \\
\hline $.5^{6}$ & 81690 & 81697 & 81704 & 81710 & 81717 & 81723 & 81730 & 81737 & 81743 & 81750 & 4 \\
\hline .57 & 81757 & 81763 & SI 770 & 81776 & $8_{1} 78_{3}$ & 81790 & 81796 & $8180_{3}$ & 81809 & 81816 & 4 \\
\hline $.5^{5}$ & 81823 & 81829 & 81836 & 81842 & 81849 & 81856 & 81862 & 81869 & 81875 & 81882 & 5 \\
\hline .59 & 81859 & 81895 & 81902 & 81908 & 81915 & 81921 & 81928 & S1 935 & 81941 & $8194^{8}$ & 5 \\
\hline 6.60 & .81954 & .81961 & .81968 & .81974 & .81981 & .81987 & .81994 & .82000 & .82007 & .82014 & 7 \\
\hline .61 & 82020 & 82027 & 82033 & 82040 & 82046 & 82053 & 82060 & 82066 & 82073 & 82079 & 1 \\
\hline .62 & 82086 & 82092 & 82099 & 82105 & 82112 & 82119 & 82125 & $\mathrm{~S}_{2} \mathrm{I}_{32}$ & 82138 & 82145 & 1 \\
\hline .63 & 82151 & $8215^{8}$ & 82164 & 82171 & 82178 & 82184 & 82191 & 82197 & 82204 & 82210 & 2 \\
\hline .64 & 82217 & 82223 & 82230 & 82236 & 82243 & 82249 & 82256 & 82263 & 82269 & 82276 & 3 \\
\hline 6.65 & .82282 & .82289 & .82295 & .82302 & .82308 & .82315 & .82321 & .82328 & .82334 & .82341 & 4 \\
\hline .66 & 82347 & 82354 & 82360 & 82367 & 82373 & 82380 & 82387 & 82393 & 82400 & 82406 & 4 \\
\hline .67 & $82+13$ & 82419 & 82426 & 82432 & 82439 & 82445 & $8245^{2}$ & $8245^{8}$ & 82465 & 82471 & 5 \\
\hline .68 & 82478 & 82484 & 82491 & 82497 & 82504 & 82510 & 82517 & 82523 & 82530 & 82536 & 6 \\
\hline .69 & 82543 & 82549 & $8255^{6}$ & 82562 & 82569 & 82575 & $825^{82}$ & 82588 & 82595 & 82601 & 6 \\
\hline 6.70 & .82607 & $.8261_{4}$ & .82620 & .82627 & .82633 & .82640 & .82646 & .82 & .82659 & .82666 & 6 \\
\hline .71 & 82672 & 82679 & 82685 & 82692 & 82698 & 82705 & 82711 & 82718 & 82724 & 82730 & I \\
\hline .72 & 82737 & 82743 & 82750 & 82756 & $8_{2} ; \sigma_{3}$ & 82769 & 82776 & 82782 & 82789 & 82795 & I \\
\hline .73 & 82802 & 82805 & 82814 & 82821 & 82827 & 82834 & 82840 & 82847 & 82853 & 82860 & 2 \\
\hline .74 & 82866 & 82872 & 82879 & 82885 & 82892 & 82898 & 82905 & 82911 & 82918 & 82924 & 2 \\
\hline 6.75 & .82930 & .82937 & .82943 & .82950 & .82956 & .82963 & .82969 & .82 & .82982 & .82988 & 3 \\
\hline .76 & 82995 & 83001 & 83008 & 83014 & 83020 & 83027 & 83033 & 83040 & 83046 & $8_{3} 0_{52}$ & 4 \\
\hline .77 & 83059 & 83065 & 83072 & 83078 & 83085 & 83091 & 83097 & $8_{3} 10_{4}$ & 83110 & 83117 & 4 \\
\hline $.7^{8}$ & 83123 & 83129 & 83136 & 83142 & 83149 & 83155 & $8316 I$ & 83168 & 83174 & 83181 & 5 \\
\hline .79 & 83187 & 83193 & 83200 & 83206 & 83213 & 83219 & 83225 & 83232 & $8323^{8}$ & 83245 & 5 \\
\hline 6.80 & .83251 & .83257 & .83264 & .83270 & .83276 & $.8328_{3}$ & .83289 & .83296 & .83302 & .83308 & 7 \\
\hline si & 83315 & 83321 & 83327 & 83334 & 83340 & 83347 & 83353 & 83359 & $8_{33} 66$ & $8337^{2}$ & I \\
\hline .82 & $8337^{8}$ & $8_{3} 3^{8} 5$ & 83391 & 83398 & 83404 & 83410 & $83+17$ & $8_{3423}$ & 83429 & 83436 & I \\
\hline .83 & $8_{3442}$ & 83448 & 83455 & 83461 & 83467 & 83474 & $8_{34} 80$ & $8_{34} s_{7}$ & 83493 & 83499 & 2 \\
\hline .84 & 83506 & 83512 & 83518 & 83525 & 83531 & 83537 & 83544 & $8355^{\circ}$ & $8355^{6}$ & $8_{35} 6_{3}$ & 3 \\
\hline 6.85 & .83569 & .83575 & $.835^{82}$ & .83588 & .83594 & .83601 &.$S_{3} 607$ & $8_{3} 61_{3}$ & .83620 &.$s_{3} 626$ & 4 \\
\hline .86 & $8_{3} 6_{32}$ & 83639 & 83645 & 83651 & $8365^{8}$ & 83664 & $8_{3670}$ & 83677 & $8_{3} 68_{3}$ & $83(68)$ & 4 \\
\hline .87 & $8_{3} 69^{6}$ & 83702 & 83708 & 83715 & $837^{21}$ & 83727 & 83734 & 83740 & 83746 & 83753 & 5 \\
\hline .88 & 83759 & 83765 & 83771 & 83778 & $8_{37} 84$ & $8_{3790}$ & 83797 & $\mathrm{~s}_{3} \mathrm{So}_{3}$ & $8_{3} S 09$ & 83816 & 6 \\
\hline .89 & 83822 & $8_{3} \mathrm{~S}_{2} \mathrm{~S}$ & $8_{3} 8_{35}$ & 83841 & 83847 & 83853 & 83560 & $8_{3} 866$ & $\delta_{3} \delta_{72}$ & 83879 & 6 \\
\hline 6.90 & $.8388_{5}$ & .83891 & .83897 & .83904 & .83910 & .83916 & .8 & .83929 & .83935 &.$s_{3}$ & 6 \\
\hline .91 & $8394^{x}$ & 83954 & 83960 & 83967 & 83973 & 83979 & 83955 & $8_{3} 092$ & $\mathrm{~s}_{3} 99^{\mathrm{s}}$ & 84004 & 1 \\
\hline .92 & 84011 & 84017 & 84023 & 84029 & 84036 & 84042 & $8_{4048}$ & 84055 & 84061 & 84067 & 1 \\
\hline .93 & 84073 & 84 oso & 84086 & 84092 & 84 ons & 84105 & 84111 & $8_{4} 117$ & $8_{4} 123$ & $\mathrm{~S}_{4} 130$ & 2 \\
\hline .94 & 84136 & 84142 & 84148 & 84155 & 84161 & 84167 & 84173 & 84180 & 84156 & 84192 & 2 \\
\hline 6.95 & .84198 & 84205 & .84211 & 84217 & 84223 & .84230 & .84236 & .84242 & .842 .48 & .84255 & 3 \\
\hline .96 & 84261 & 84267 & 84273 & 84250 & 84286 & $S_{4} 292$ & $8.20 \mathrm{~s}$ & $\mathrm{~S}_{4} 305$ & $S_{4} 311$ & 84317 & 4 \\
\hline .07 & 84323 & $8433^{\circ}$ & 843.36 & 84342 & 84348 & 84354 & 84361 & 84.367 & 84373 & 84379 & 4 \\
\hline $.9^{8}$ & 84386 & 84302 & 84308 & 84404 & 84410 & 54417 & $S_{4423}$ & $S_{4} 430$ & 844.35 & $s_{4442}$ & 5 \\
\hline 99 & 84448 & 84454 & 844 & 84466 & 84473 & 84479 & $\mathrm{~S}_{44} \mathrm{~S}_{5}$ & 84491 & 84497 & $\mathrm{~S}_{4} 5 \mathrm{O}_{4}$ & 5 \\
\hline
\end{tabular}




\section{FIVE PLACE LOGARITHMS.}

LOGS. 5 PL.

\begin{tabular}{|c|c|c|c|c|c|c|c|c|c|c|c|}
\hline No. & 0 & I & 2 & 3 & 4 & 5 & 6 & 7 & 8 & 9 & $\begin{array}{l}\text { INTP } \\
\text { TAB. }\end{array}$ \\
\hline 7.00 & .84510 & $.845^{16}$ & .84522 & .84528 & .84535 & $.8454 \mathrm{I}$ & .84547 & .84553 & 59 & .84566 & 7 \\
\hline .or & $8457^{2}$ & 84578 & $8_{4} 5^{S_{4}}$ & 84590 & 84597 & 84603 & 84609 & 84615 & & 84628 & I \\
\hline .02 & $8_{4} 634$ & 84640 & 84646 & $\mathrm{~S}_{4} 6_{52}$ & 84658 & 84665 & 84671 & 84677 & $8_{4} 68_{3}$ & 84689 & I \\
\hline .03 & 84696 & 84702 & 84708 & 84714 & 84720 & 84726 & 84733 & 84739 & 84745 & $8_{4} 75^{I}$ & 2 \\
\hline .04 & 84757 & $847^{6} 3$ & 84770 & 84776 & 84782 & 84788 & 84794 & 84800 & $84 \mathrm{SO}_{7}$ & 84813 & 3 \\
\hline 05 & .84819 & .84825 & .84831 & .84837 & .84844 & .84850 & .84856 & .84862 & .84868 & 4874 & 4 \\
\hline 06 & $8_{4} 980$ & $8_{4} 88_{7}$ & 84893 & 84899 & & 84911 & 84917 & 84924 & & 4936 & 4 \\
\hline .07 & 84942 & 84948 & 84954 & 84960 & 84 & 84973 & 84979 & 84985 & 8499 I & 4997 & 5 \\
\hline .08 & 85003 & 85009 & 85016 & 85022 & 85028 & 85034 & 85040 & 85046 & 85052 & $505^{8}$ & 6 \\
\hline .09 & 85065 & 85071 & 85077 & $8_{5} 083$ & 85089 & 85095 & $85 \mathrm{IOI}$ & 85107 & 85114 & 120 & 6 \\
\hline 7.10 & .85126 & .85132 & .85138 & .85144 & 50 & 156 & & .85169 & .85175 & .85 I 81 & 6 \\
\hline .II & 85187 & 85193 & 85199 & 85205 & 85211 & 85217 & 85224 & 85230 & $8523^{6}$ & 85242 & I \\
\hline .12 & 85248 & 85254 & 85260 & 85266 & 85272 & 85278 & 85285 & 85291 & 85297 & 85303 & I \\
\hline .13 & 85309 & 85315 & 85321 & 85327 & 85333 & 85339 & 85345 & $8535^{2}$ & $8535^{8}$ & 85364 & 2 \\
\hline .14 & 85370 & 85376 & 85382 & 85388 & 85 & & 85406 & 85412 & 85 & 25 & 2 \\
\hline 7.15 & .85431 & .85437 & .85 & .85449 & .8 & $5 \mathbf{I}$ & .85467 & .85473 & .85 & 85 & 3 \\
\hline .16 & 85491 & 85497 & 85503 & 85509 & 85 & & 85528 & 85534 & 40 & 85546 & 4 \\
\hline .17 & $8555^{2}$ & $8555^{8}$ & 85564 & 85570 & 85576 & $855^{82}$ & 85588 & 85594 & 85600 & 85606 & 4 \\
\hline .18 & 85612 & 85618 & 85625 & 85631 & 85637 & 85643 & 85649 & 85655 & $8566 r$ & 85667 & 5 \\
\hline .19 & 85673 & 85679 & 85685 & 85691 & 85697 & 85703 & 85709 & 85715 & 85721 & 85727 & 5 \\
\hline 7.20 & .85 & 39 & 3 & .0 &. & $.857^{6} 3$ & 769 & & 81 & 8 & 6 \\
\hline .21 & 85794 & 85800 & 85806 & 85812 & 85 & 85824 & $8_{5} 830$ & 85836 & 85842 & & I \\
\hline .22 & 85854 & 85860 & 85866 & 85872 & 85878 & 85884 & 85890 & 85896 & 85 & 85 & I \\
\hline .23 & 85914 & 85920 & 85926 & 85932 & 859 & 85944 & 85950 & 85956 & 85962 & 68 & 2 \\
\hline .24 & .85974 & 85980 & 85986 & 85992 & & & & & & & 2 \\
\hline 7.25 & .86034 & .86040 & .86046 & .86052 & $.8605^{8}$ & .86064 & .86070 & $.86 \circ 0^{6}$ & .86082 & 88 & 3 \\
\hline .26 & 86094 & 86100 & 86 Iо6 & 86 I1 2 & 86118 & 86124 & 30 & 86 I 36 & $y$ & & 4 \\
\hline .27 & 86 I53 & $86 \times 59$ & 86165 & 86 I 7 I & 86 & 86183 & 86189 & 95 & & & 4 \\
\hline .28 & 86213 & 86219 & 86225 & 86231 & 862 & 86243 & 86249 & 86255 & $8626 r$ & 67 & 5 \\
\hline .29 & 86273 & 86279 & 85 & 86291 & 86 & 3 & 308 & 86 & 20 & 26 & 5 \\
\hline 7.30 & .86332 & $.8633^{8}$ & .8 & & & & & & & & 6 \\
\hline .31 & 86392 & 86398 & 86404 & 864 & 86 & II & 27 & 33 & & & I \\
\hline .32 & $8645^{1}$ & 86457 & 86463 & 86469 & 86475 & 8 & $864^{87}$ & 86 & 99 & 04 & I \\
\hline .33 & 86510 & 86516 & 86522 & 86528 & 86534 & 40 & 86546 & 86552 & $8655^{8}$ & 64 & 2 \\
\hline .34 & 86570 & 86576 & $8658 \mathrm{I}$ & $865^{87}$ & 86593 & 99 & 86605 & 86 6II & 17 & 623 & 2 \\
\hline $7 \cdot 35$ & .86629 & .86635 & $.8664 \mathrm{I}$ & .86646 & .86652 & .86658 & .86664 & .86670 & .86676 & .86682 & 3 \\
\hline .36 & 86688 & 86694 & 86700 & 86705 & & & 86723 & 29 & & & 4 \\
\hline .37 & 86747 & 86753 & 86759 & 86764 & 86770 & 86776 & 86782 & 86788 & 86794 & 800 & 4 \\
\hline .38 & 86806 & 86812 & 86817 & 86823 & 86 & 86835 & $8684 I$ & 86847 & 86853 & 86859 & 5 \\
\hline$\cdot 39$ & 86864 & 86870 & 86876 & 86882 & & 86 & 86900 & 6 & 86911 & 86917 & 5 \\
\hline 7.40 & .869 & .86 & & & & & & & & & 5 \\
\hline & 86982 & 86988 & 86 & 86 & 87 & 87 & 87 & 87 & 87 & 87 & I \\
\hline 42 & 87040 & 87046 & 87052 & $8705^{8}$ & 87 & 87 & 87075 & 87081 & 87087 & 93 & I \\
\hline 43 & 87099 & 87105 & 87 III & 87116 & 87 & 87 & 87134 & 87 & 87 & & 2 \\
\hline 44 & 87157 & 87163 & 87169 & 87175 & 87 I 81 & 87186 & 87192 & 87198 & 87204 & 87210 & 2 \\
\hline 7.45 & .87216 & .87221 & .87 & .87233 & .87 & .87 & $.8725 \mathrm{I}$ & .87256 & .87 & .87 & 3 \\
\hline .46 & 87274 & 87280 & 87286 & 87291 & .87297 & & 87309 & 87315 & 87320 & 87326 & 3 \\
\hline .47 & 87332 & $8733^{8}$ & 87344 & 873 & 873 & 87361 & 87367 & 87373 & 87379 & 87384 & 4 \\
\hline .48 & 87390 & 87396 & & 874 & 87413 & 87419 & 87425 & & 87437 & 87 & 4 \\
\hline .49 & 87448 & 87454 & 87460 & 87466 & 87471 & 87477 & 87483 & 87489 & 87495 & 87500 & 5 \\
\hline
\end{tabular}




\begin{tabular}{|c|c|c|c|c|c|c|c|c|c|c|c|}
\hline No. & 0 & I & 2 & 3 & 4 & 5 & 6 & 7 & 8 & 9 & $\begin{array}{l}\text { INTP. } \\
\text { TAB. }\end{array}$ \\
\hline 7.50 & .87506 & .87512 & .87518 & .87523 & .87529 & .87535 & .87541 & .87547 & $.8755^{2}$ & $.8755^{8}$ & 6 \\
\hline .51 & 87564 & 87570 & 87576 & 87581 & $875^{87}$ & 87593 & 87599 & 87604 & 87610 & 87616 & I \\
\hline .52 & 87622 & $s_{7} 628$ & 87633 & $s_{7} 639$ & $S_{7} 645$ & 87651 & 87656 & 87662 & 87668 & 87674 & I \\
\hline .53 & 87679 & 87685 & 87691 & 87697 & 87703 & 87708 & 87714 & 87720 & 87726 & 87731 & 2 \\
\hline .54 & 87737 & 87743 & 87749 & 87754 & 87760 & 87766 & 87772 & 87777 & 87783 & 87789 & 2 \\
\hline 7.53 & .87795 & .87800 & .87806 & .87812 & .87818 & .87823 & .87829 & .87835 & .87841 & .87846 & 3 \\
\hline .56 & $8_{7} S_{52}$ & $S_{7} s_{5} 8$ & 87864 & 87869 & 87875 & $8788 \mathrm{I}$ & 87887 & 87892 & 87898 & 87904 & 4 \\
\hline .57 & 87910 & $8_{7915}$ & 87921 & 87927 & 87933 & $8793^{8}$ & 87944 & 87950 & 87955 & 87961 & 4 \\
\hline .53 & 87967 & 87973 & 87978 & 87984 & 87990 & 87996 & 88001 & 88007 & 88 or 3 & 88018 & 5 \\
\hline .59 & SS 024 & 88 o30 & 88036 & $880_{41}$ & 88047 & 88053 & 88058 & 88064 & 88070 & 88076 & $\begin{array}{l}5 \\
5\end{array}$ \\
\hline 7.60 &.$S S$ oSI & .88087 & .85093 & $.88 \circ 98$ & .88104 & .88 I 10 & .88 I16 & .85121 & .88127 & .88133 & 5 \\
\hline .61 & 88138 & 88144 & 88150 & 88 I56 & 88161 & 88167 & 88173 & 88178 & 88184 & 88190 & I \\
\hline .62 & 88195 & 88201 & 88207 & 88213 & 88218 & 88224 & 88230 & 88235 & 88241 & 88247 & I \\
\hline .63 & 88252 & $8825^{8}$ & 88264 & 88270 & 88275 & 88281 & 88287 & 88292 & 88298 & 88304 & 2 \\
\hline .64 & 88309 & 88315 & 88321 & 88326 & 88332 & $8833^{8}$ & 88343 & 88349 & 88355 & 88360 & 2 \\
\hline. .65 & .88366 & .88372 & .88377 & $.88_{3} 8_{3}$ & .88389 & .88395 & .88400 & .88406 & .88412 & .88417 & 3 \\
\hline .66 & $88_{423}$ & 88429 & 88434 & 88440 & 88446 & $8845^{1}$ & 88457 & $88_{4} 6_{3}$ & 88468 & $88+74$ & 3 \\
\hline .67 & $\mathrm{SS}_{4} 8 \mathrm{O}$ & 88485 & 88491 & $88+97$ & 88502 & 88508 & $885^{1} 3$ & 88519 & 88525 & 88530 & 4 \\
\hline .68 & 88536 & 88542 & 88547 & 88553 & 88559 & 88564 & 88570 & 88576 & $885^{8 i}$ & $\mathrm{SS}_{5}{ }^{87}$ & 4 \\
\hline .69 & 88593 & 88598 & 88604 & 88610 & 88615 & 88621 & 88627 & 88632 & 88638 & 88643 & 5 \\
\hline 7.70 & .88649 & .88655 & .88660 & .88666 & .88672 & .88677 & $.8868_{3}$ & .88689 & .88694 & .88700 & 6 \\
\hline .71 & $\mathrm{Ss}_{7} \mathrm{O}_{5}$ & 88711 & 88717 & 88722 & 88728 & 88734 & 88739 & 88745 & 88750 & $8875^{6}$ & I \\
\hline .72 & $887(62$ & 88767 & 88773 & 88779 & 88784 & 88790 & 88795 & 88 SoI & 88807 & SSS12 & I \\
\hline .73 & 88818 & 88824 & 88829 & $888_{35}$ & 88840 & 88846 & 88852 & $88 S_{57}$ & $8886_{3}$ & $88 \$ 68$ & 2 \\
\hline .74 & 88874 & 88880 & 88885 & 88891 & $88 \mathrm{~S} 97$ & 88902 & 88908 & 88913 & 88919 & 88925 & 2 \\
\hline 7.75 & .88930 & .88936 & .88941 & .88947 & .88953 & $.8895^{8}$ & .88964 & .88969 & .88975 & .8898 r & 3 \\
\hline-76 & $889 \$ 6$ & $8 S_{992}$ & 88997 & 89003 & 89009 & 89014 & 89020 & 89025 & 89031 & $\mathrm{~S}_{9} \mathrm{O}_{37}$ & 4 \\
\hline .77 & 89042 & 8r) 048 & 89053 & 89059 & 89064 & 89070 & 89076 & S9 08i & 89087 & $\mathrm{Sg}_{9} \mathrm{O}_{92}$ & 4 \\
\hline .78 & $8900 \mathrm{~s}$ & 89) 104 & 89109 & 89115 & $89 \times 20$ & 89126 & 89131 & 89137 & 89143 & 89148 & 5 \\
\hline .79 & 89154 & S9 159 & 89165 & 89170 & 89176 & 89182 & 89187 & 89193 & 89198 & 89204 & 5 \\
\hline 7.80 & .59209 & So 215 & .89221 & .89226 & .89232 & .S9 237 & .89243 & .89248. & .89254 & .89260 & 5 \\
\hline 81 & $S_{9} 265$ & 89271 & 89276 & S9 282 & 89287 & 89293 & 89298 & 89304 & 89310 & 89315 & I \\
\hline .82 & Su) 321 & 8) 326 & 8) 332 & 89337 & 89343 & S9 $34^{8}$ & 89) 354 & $\operatorname{sg}_{3} 60$ & 89365 & $8937 \mathrm{I}$ & I \\
\hline 83 & So 376 & $\mathrm{So}_{3} \mathrm{~S}_{2}$ & $893^{87}$ & 89 393 & 89398 & 89404 & 89409 & $S_{9}+15$ & 89421 & 89426 & 2 \\
\hline .84 & $S_{9} 43^{2}$ & S9 437 & 89443 & 89448 & 89454 & Si) 459 & $\mathrm{~S}_{9} 4 \mathrm{O}_{5}$ & 89470 & 89476 & 89481 & 2 \\
\hline 7.85 & So 487 & .89492 & $.8949^{8}$ & .89504 & .89509 & .89515 & S9 520 & .89526 & .89531 &.$S 95.37$ & 3 \\
\hline .86 & $S_{9} 542$ & $8954^{8}$ & S9 553 & 89 559 & 8) 564 & 89570 & 89575 & $895^{81}$ & 89586 & 89592 & 3 \\
\hline$s_{7}$ & Sig 597 & $8 y \mathrm{KO}_{3}$ & Sofron & $\mathrm{Sg}_{914}$ & 89620 & 89625 & 80631 & $\mathrm{sig}_{9} 6$ & 89642 & S9 647 & 4 \\
\hline .ss & So 653 & So $65 \mathrm{~s}$ & $\mathrm{Sg} 664$ & So) $6(x)$ & 89675 & 89680 & 89686 & $89(x) 1$ & 89697 & 89702 & 4 \\
\hline .89 & S9 708 & 89713 & 89719 & S9 724 & 89730 & 89735 & 89741 & S9 746 & $8975^{2}$ & 89757 & 5 \\
\hline 7.90 & $.897^{6} 3$ & .89768 & .89774 & S9 779 & $.897_{5}$ & .89790 & .89796 & So Sor & .89807 & .80912 & 6 \\
\hline .) 1 & Sigs sis & $\mathrm{Sig}_{23}$ & $8082 y$ & $S_{y} 8_{34}$ & $\mathrm{~S}_{9} \mathrm{~S}_{40}$ & $898_{45}$ & $80 S_{51}$ & 89856 & 89862 & $80 \$ 67$ & I \\
\hline .92 & $\mathrm{~S}_{0} \mathrm{~S}_{73}$ & $\mathrm{~S}_{9} \mathrm{~S}_{7} \mathrm{~S}$ & $\mathrm{SoSs}_{3}$ & 80889 & 80894 & S9 900 & $\mathrm{Sog}_{9}$ & So git & 89916 & 80922 & 1 \\
\hline .93 & Si, 927 & 80933 & 879.38 & 89944 & So 949 & S9.955 & 89910 & 89966 & 89971 & 89977 & 2 \\
\hline .94 & $809 \$ 2$ & 89958 & 89093 & S9 pos & 90004 & 90009 & 90015 & 90020 & 90026 & 90031 & 2 \\
\hline 7.95 & .90037 & .90042 & .900 .48 & .90053 & .90059 & .90064 & $.90 \mathrm{ol}(\mathrm{s})$ & .90075 & .90080 & .90086 & 3 \\
\hline .96 & 90091 & 90097 & 90102 & 90108 & 20113 & (90 III) & 90124 & 90120 & 90135 & 90140 & 4 \\
\hline .97 & 20146 & 90151 & 90157 & 9016,2 & 90168 & 90173 & 90179 & 90184 & 90189 & 90195 & 4 \\
\hline $0 \mathrm{~s}$ & 90200 & $90 \mathrm{an}$ & 90211 & 90217 & 90222 & 90227 & 90233 & $m=38$ & 90244 & 90249 & 5 \\
\hline .99 & 90255 & 90260 & 90266 & 90271 & 90276 & 90282 & 90287 & 90203 & 90298 & 90.304 & 5 \\
\hline
\end{tabular}


8. FIVE PLACE LOGARITHMS.

LOGS. 5 PL.

.90

\begin{tabular}{|c|c|c|c|c|c|}
\hline No. & 0 & $\mathbf{I}$ & 2 & 3 & 4 \\
\hline 00 & .90309 & $.903^{14}$ & .90320 & .90325 & $.9033 \mathrm{I}$ \\
\hline & 90363 & 90369 & 90374 & 90380 & 90385 \\
\hline .02 & 90417 & 90423 & 90428 & 90434 & 90439 \\
\hline .03 & $9047^{2}$ & 90477 & 90482 & 90488 & 90493 \\
\hline .04 & 90526 & 90531 & $9053^{6}$ & 90542 & 90547 \\
\hline 8.05 & .90580 & .90585 & .90590 & .90596 & .90601 \\
\hline & 90634 & 90639 & 90644 & 90650 & 90655 \\
\hline & 90687 & 90693 & 90698 & 90703 & 90709 \\
\hline . 08 & 90741 & 90747 & $9075^{2}$ & 90757 & 90763 \\
\hline .09 & 90795 & 90800 & 90806 & 90811 & 90816 \\
\hline
\end{tabular}

8.10 .90 849.90854 .90859 .90865 .90870 .II $9090290907 \quad 9091390918 \quad 90924$

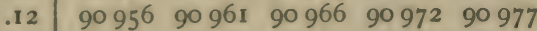
.r3 91009 91 014 91 O20 91 025 91030 $\begin{array}{llllllllll}.14 & 91062 & 91 & 068 & 91073 & 91078 & 91084\end{array}$

$8.15 \quad .91116 .91121 .91126 .91132 .91137$ $\begin{array}{llllllllll}.16 & 91169 & 91 & 174 & 91 & 180 & 91 & 185 & 91 & 190\end{array}$

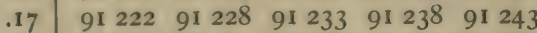

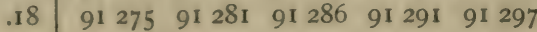

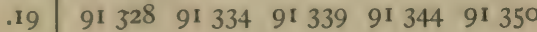

8.20 .21 .22 .23 .24 8.25

.26

.27

.28

.29

8.30

.31

.32

.33

.34

8.35

.36

.37

.38

.39

8.40

$.4 \mathrm{I}$

.42

.43

.44

8.45

.90

.95
.91 381.91 387.91392 .91397 .91402 91434 91 440 91 445 91 450 91 455 $9148791492 \quad 91498 \quad 91503 \quad 91508$ 91 540 9I 545 9I 55 I 91 I $55^{6}$ 91 56 I 91 593 91 598 91 603 91 609 91 614 .91 645 .91 651 I 9 .91 656 .91 661 I 666 91 698 91 703 91 709 91 714 91 719 91 751 91756 9I 761 91 766 91 772

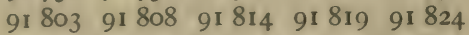
91 855 91 86 I 91866 9I 871 9ा 876

.91908 .91913 .91918 .91924 .91929 91960 91 965 91 971 91 976 91 98I $\begin{array}{llllll}92012 & 92018 & 92023 & 92028 & 92033\end{array}$ $\begin{array}{lllll}92065 & 92070 & 92075 & 92080 & 92085\end{array}$

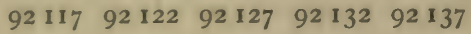
$.92169 .92 \times 74 \quad .92179 .92184 .92189$

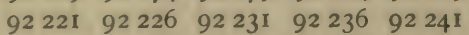
$\begin{array}{llllll}92 & 273 & 92278 & 92283 & 92288 & 92293\end{array}$ $92324 \quad 92330 \quad 92335 \quad 9234092345$

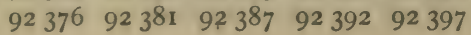

.92428 .92433 .92438 .92443 .92449 $\begin{array}{llllll}92480 & 92485 & 92490 & 92495 & 92500\end{array}$ $\begin{array}{lllllll}92531 & 92536 & 92542 & 92547 & 92552\end{array}$

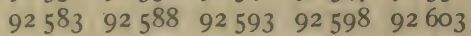
$\begin{array}{llllll}92634 & 92639 & 92645 & 92650 & 92655\end{array}$ $.92686 .92691 \quad .92696 .92701 \quad 92706$ $\begin{array}{ll}92737 \quad 92742 \quad 92747 \quad 9275^{2} & 9275^{8}\end{array}$ $\begin{array}{llllll}92788 & 92793 & 92799 & 92804 & 92809\end{array}$ $\begin{array}{lllllll}92840 & 92845 & 92850 & 92855 & 92860\end{array}$ $92891 \quad 92896 \quad 92901 \quad 92906929 \mathrm{II}$

$\begin{array}{llllll}5 & 6 & 7 & 8 & 9\end{array}$

.90336 .90342 .90347 .90352 .90358 9039090396904019040790412 $90445 \quad 90450 \quad 90455 \quad 90461 \quad 90466$ $90499 \quad 90504 \quad 90509 \quad 90515 \quad 90520$

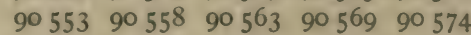
.90607 .90612 .90617 .90623 .90628 $90660 \quad 90666 \quad 90671 \quad 90677 \quad 90682$ $90714 \quad 90720 \quad 90725 \quad 9073090736$

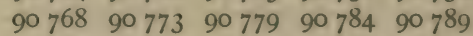
$\begin{array}{llllll}90822 & 90827 & 90832 & 90 & 838 & 90843\end{array}$

.90875 .90881 r.90 886.90891 .90897 $9092990934 \quad 9094090945 \quad 90950$ $90982909889099390998 \quad 91004$ $\begin{array}{llllllllllllllll}91036 & 91041 & 91 & 046 & 91052 & 91057\end{array}$ 91089 91 094 9r roo 91 105 91 x10

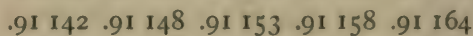

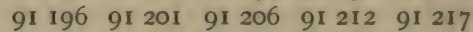
9I 249 9I 254 9I 259 9I 265 9I 270 91 $302 \quad 91307$ 91 $31291318 \quad 91323$ 91 355 91 360 91 365 91 371 91 376

.91 408.91413 .91418 .91424 .91420

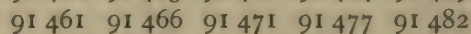
91 $514 \quad 915199152491529$ 91 535 91 566 91 572 91 577 91 582 91 587 9r 619 9r 624 9r 630 9r 635 91 640 .91 672 .91 677.91682 .91687 .91693

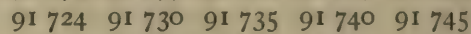
91 777 91 782 91 787 91 793 91 798 91 829 91 $834 \quad 91840$ 91 $845 \quad 91850$ 91 882 91 887 91 892 91 897 91 903

91934.91939 .91944 .91950 .95195

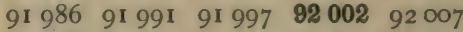
$\begin{array}{llllll}92038 & 92044 & 92049 & 92054 & 92059\end{array}$ 920919209692 IOI 92 I06 92 III $\begin{array}{lllllll}921143 & 92148 & 92.153 & 92158 & 92 & 163\end{array}$ .92195 .92200 .92205 .92210 .92215

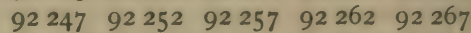

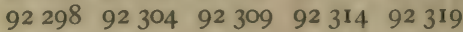

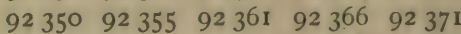
$\begin{array}{lllllll}92402 & 92407 & 92 & 412 & 92418 & 92 & 423\end{array}$ .92454 .92459 .92464 .92469 .92474 $9250592511 \quad 92516 \quad 92521 \quad 92526$

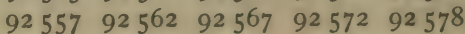
$\begin{array}{llllll}92609 & 92614 & 92619 & 92624 & 92629\end{array}$ $\begin{array}{lllllll}92660 & 92665 & 92670 & 92675 & 92681\end{array}$ .92711 .92716 .92722 .92727 .92732

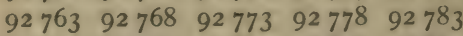
$\begin{array}{llllllll}92814 & 92819 & 92824 & 92829 & 92834\end{array}$ $\begin{array}{lllllll}92865 & 92.870 & 92875 & 92881 & 92886\end{array}$ $92.916 \quad 92921 \quad 92927 \quad 9293292937$ $\mid \begin{aligned} & \text { INTP. } \\ & \text { TAB. }\end{aligned}$

5

1

3

3

4 5

6

1

I

2

3

4 4 5 5 I I 2 3 3 4 4 5 6 I I 2 3 4
4 5 5 5 I 2 2 3 3
4 4
5 


\begin{tabular}{|c|c|c|c|c|c|c|c|c|c|c|c|}
\hline No. & 0 & I & 2 & 3 & 4 & 5 & 6 & 7 & 8 & 9 & $\begin{array}{l}\text { INTP } \\
\text { TAB. }\end{array}$ \\
\hline 8.50 & .92942 & .92947 & .92952 & .92957 & .92962 & .92967 & .92973 & .92978 & $.929 S_{3}$ & $.929 S S$ & 6 \\
\hline .51 & 92993 & 92998 & 93003 & 93008 & 93013 & 93018 & 93024 & 93029 & 93034 & 93039 & I \\
\hline .52 & 93044 & 93049 & 93054 & 93059 & 93064 & 93069 & 93075 & 93080 & 93085 & 93090 & I \\
\hline .53 & 93095 & 93100 & 93105 & 93110 & 93115 & 93120 & 93125 & 93131 & 93136 & 93141 & 2 \\
\hline .54 & 93146 & $9315^{1}$ & 93156 & 93161 & 93166 & 93171 & 93176 & 93181 & 93186 & 93192 & 2 \\
\hline$\$ .55$ & .93197 & .93202 & .93207 & .93212 & .93217 & .93222 & .93227 & .93232 & .93237 & .93242 & 3 \\
\hline .56 & 93247 & 93252 & 93258 & 93263 & 93268 & 93273 & 93278 & 93283 & 93288 & 93293 & 4 \\
\hline .57 & 93298 & $933_{3}$ & 93308 & $933^{1} 3$ & 93318 & 93323 & 93328 & 93334 & 93339 & 93344 & 4 \\
\hline $.5^{5}$ & 93349 & 93354 & 93359 & 93364 & 93369 & 93374 & 93379 & $933^{8} 4$ & 93389 & 93394 & 5 \\
\hline .59 & 93399 & 93404 & 93409 & 93414 & 93420 & 93425 & 93430 & 93435 & 93440 & 93445 & 5 \\
\hline 8.60 & $.93+50$ & .93455 & .93460 & .93465 & .93470 & .93475 & .93480 & .93485 & .93490 & .93495 & 5 \\
\hline .61 & 93500 & 93505 & 93510 & 93515 & 93520 & 93526 & $9353^{1}$ & 93536 & $9354 I$ & 93546 & I \\
\hline .62 & 93551 & 93556 & 93561 & 93566 & $9357^{1}$ & 93576 & $935^{81}$ & 93586 & 93591 & 93596 & I \\
\hline .63 & 93601 & 93606 & 93611 & 93616 & 93621 & 93626 & 93631 & 93636 & 93641 & 93646 & 2 \\
\hline .64 & 93651 & 93656 & 93661 & 93666 & 93671 & 93676 & 93682 & 93687 & 93692 & 93697 & 2 \\
\hline S.65 & .93702 & .93707 & .93712 & .93717 & .93722 & .93727 & .93732 & .93737 & .93742 & .93747 & 3 \\
\hline .66 & 93752 & 93757 & 93762 & 93767 & 93772 & 93777 & $937 S_{2}$ & 93787 & 93792 & 93797 & 3 \\
\hline .67 & $93 \mathrm{SO}_{2}$ & $93 \mathrm{SO}_{7}$ & 93812 & 93817 & 93822 & 93827 & $93 \$_{32}$ & $938_{37}$ & 93842 & 93847 & 4 \\
\hline .68 & $93 \mathrm{~S}_{52}$ & 93857 & $93 \$ 62$ & 93867 & 93872 & 93877 & 93882 & 93887 & $93 \mathrm{Sg} 2$ & 93897 & 4 \\
\hline .69 & 93902 & 93907 & 93912 & 93917 & 93922 & 93927 & 93932 & 93937 & 93942 & 93947 & 5 \\
\hline 8.70 & $.9395^{2}$ & .93957 & .93962 & .93967 & .93972 & .93977 & $.939 \$_{2}$ & .93987 & & .93 & 4 \\
\hline .71 & 94002 & 94007 & 94012 & 94017 & 94022 & 94027 & 94032 & 94037 & 94042 & 94047 & 0 \\
\hline .72 & 94052 & 94057 & 94062 & 94067 & 94072 & 94077 & 94082 & 94086 & 94091 & 94096 & I \\
\hline .73 & 94101 & 94106 & 94111 & 94 II 6 & $94 \mathbf{I 2 1}$ & 94126 & 94131 & $94 \quad 136$ & 94141 & 94146 & I \\
\hline .74 & 94 I $5 I$ & 94156 & 94 I6I & 94166 & 94171 & 94176 & 94181 & $941 \$ 6$ & 94191 & 94196 & 2 \\
\hline S. 75 & .94201 & .94206 & .94211 & .94216 & .94221 & .94226 & .94231 & .94236 & .94240 & .94245 & 2 \\
\hline $.7^{6}$ & 94250 & 94255 & 94260 & 94265 & 94270 & 94275 & 94280 & $9+285$ & 94290 & 94295 & 2 \\
\hline .77 & 94300 & 94305 & 94310 & 94315 & 94320 & 94325 & 94330 & 94335 & 94340 & 94345 & 3 \\
\hline $.7^{5}$ & 94349 & 94354 & $9+359$ & 94364 & 94369 & 94374 & 94379 & 94384 & $943^{\mathrm{So}}$ & 94394 & 3 \\
\hline .79 & 94399 & 94404 & 94409 & $944^{14}$ & 94419 & 94424 & 94429 & 94433 & 94438 & 94443 & 4 \\
\hline 8.80 & $.94+48$ & .94453 & $.9445^{8}$ & .94463 & .94468 & .94473 & $.9447^{-S}$ & .94483 & .94488 & .94493 & 5 \\
\hline .81 & 24498 & 94503 & 94507 & 94512 & 94517 & $945^{22}$ & 94527 & 94532 & 94537 & 94542 & I \\
\hline .82 & 94547 & 94552 & 94557 & $945^{62}$ & 94567 & 94571 & 94576 & 94581 & 94556 & 94591 & I \\
\hline.$S_{3}$ & 94596 & 94601 & 94606 & 94611 & 94616 & 94621 & 94626 & 94630 & 94635 & 94640 & 2 \\
\hline .84 & 94645 & 94650 & 94655 & 94660 & 94665 & 94670 & 94675 & 94650 & $9468_{5}$ & 94689 & 2 \\
\hline $8.8_{5}$ & .94604 & .94609 & .94704 & .94709 & .94714 & .94719 & .94724 & .94729 & .94734 & $.9473^{5}$ & 3 \\
\hline .86 & 94743 & 94748 & 94753 & $9475^{\mathrm{S}}$ & $947^{6} 3$ & 94768 & 94773 & $9477^{\mathrm{S}}$ & $94-53$ & $947^{37}$ & 3 \\
\hline .87 & 94792 & 94707 & $94 \mathrm{SO}_{2}$ & $94 \therefore 07$ & $94 \$ 12$ & $948: 7$ & $94 \delta_{22}$ & $94 \mathrm{~S}_{27}$ & $94 \$_{j 2}$ & $04 \$_{36}$ & 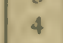 \\
\hline .85 & $94 S_{41}$ & 94846 & $94 S_{51}$ & 94856 & 94861 & 94866 & 94871 & 94876 & 94550 & $94 \mathrm{SS}_{5}$ & 4 \\
\hline$s_{9}$ & 94890 & 94895 & 94900 & 94905 & 94910 & 94915 & 94919 & 94924 & 94929 & 94934 & 5 \\
\hline 8.90 & .949 .39 & .04944 & .24049 & & .94959 & .94963 & .94968 & .94973 & $0497^{-8}$ & $.9498_{3}$ & 4 \\
\hline .91 & 94058 & $94 \mathrm{~m}_{3}$ & 94098 & 95002 & 95007 & 95012 & 95017 & 95022 & 95027 & 950,32 & 0 \\
\hline .92 & 95036 & $95 \cap 41$ & 95046 & 95051 & 95056 & 95061 & 95066 & $950 ; 1$ & 95075 & 950 oso & 1 \\
\hline 93 & 95 os 5 & 95090 & 95095 & 95100 & 95105 & 95100 & 95114 & 95119 & 95124 & 95129 & I \\
\hline .94 & 95134 & $95 \quad 139$ & 95143 & 951.48 & $95 \quad 153$ & $9515^{8}$ & 95163 & 95165 & 95173 & 95177 & 2 \\
\hline 8.95 & .95182 & .95187 & .95192 & .95197 & .95202 & .95207 & .95211 & .95216 & .95221 & .05226 & 2 \\
\hline .96 & 95231 & 95236 & 95240 & 95245 & 95250 & 95255 & 95260 & 95265 & 95270 & 95274 & 2 \\
\hline .97 & 95279 & $952 R_{4}$ & $952 S_{9}$ & 95.244 & 95290 & 95303 & $95.30 \mathrm{~s}$ & 95313 & 953 is & 95323 & 3 \\
\hline .28 & 95328 & 05,332 & 95337 & 95.342 & 95347 & 95,352 & 95357 & $953^{61}$ & $953^{(x)}$ & 95371 & 3 \\
\hline 99 & 95376 & $953^{81}$ & 95356 & 95390 & 95395 & 95400 & 95405 & 95410 & $95+15$ & 95419 & 4 \\
\hline
\end{tabular}




\begin{tabular}{|c|c|c|c|c|c|c|c|c|c|c|c|}
\hline No. & 0 & I & 2 & 3 & 4 & 5 & 6 & 7 & 8 & 9 & $\begin{array}{l}\text { INTP } \\
\text { TAB. }\end{array}$ \\
\hline 9.00 & .95424 & .95429 & .95434 & .95439 & .95444 & .95448 & .95453 & $.9545^{8}$ & .95463 & .95468 & 5 \\
\hline .01 & 95472 & 95477 & 95482 & 95487 & 95492 & 95497 & & 95506 & & & I \\
\hline .02 & 95521 & 95525 & 95530 & 95535 & 95540 & 95545 & 95550 & 95554 & 95559 & 95564 & I \\
\hline .03 & 95569 & 95574 & $9557^{8}$ & $955^{8} 3$ & 95588 & 95593 & 95598 & 95602 & 95607 & 95612 & 2 \\
\hline .04 & 95617 & 95622 & 95626 & $9563 I$ & 95636 & $9564 \mathrm{I}$ & 95646 & 95650 & 95655 & 95660 & 2 \\
\hline 9.05 & .95665 & .95670 & .95674 & .95679 & .95684 & .95689 & .95694 & .95698 & .95703 & .95708 & 3 \\
\hline .06 & 95713 & 95718 & 95722 & 95727 & 95732 & 95737 & 95742 & 95746 & $9575^{1}$ & 95756 & 3 \\
\hline .07 & $9576 \mathrm{r}$ & 95766 & 95770 & 95775 & 95780 & 95785 & 95789 & 95794 & 95799 & 95 & 4 \\
\hline .08 & 95809 & 95813 & 95818 & 95823 & 95828 & 95832 & 95837 & 95842 & 95847 & 95852 & 4 \\
\hline .09 & 95856 & $9586 \mathrm{I}$ & 95866 & 95871 & 95875 & 95880 & 95885 & 95890 & 95895 & 95899 & 5 \\
\hline 9.10 & .95904 & .95909 & .95914 & .95 & .95 & .95928 & .95 & & 12 & 47 & 4 \\
\hline .11 & $9595^{2}$ & 959 & $9596 \mathbf{I}$ & 95966 & 95 & 95 & 95980 & & & & 0 \\
\hline .12 & 95999 & 96004 & 96009 & 96014 & 96019 & $96 c$ & 96028 & $96 \circ 33$ & & & I \\
\hline .13 & 96047 & 96052 & 96057 & 96061 & 96066 & 96 & $96 \circ 76$ & 96080 & 96085 & & I \\
\hline .14 & 96095 & 96099 & 96104 & 96109 & 96114 & 96 & 96123 & 96 I 28 & $96 \div 33$ & 96 1 37 & 2 \\
\hline 9.15 & .96142 & .96147 & $.9615^{2}$ & .96156 & .961 & .96166 & .96 & .9 & .96180 & .96 I 85 & 2 \\
\hline .16 & 96190 & $96 \mathrm{I}$ & 96199 & 96204 & 962 & 96 & & & 96227 & & 2 \\
\hline .17 & 96237 & 96242 & 96246 & 96251 & 962 & 96 & 96 & 96 & 96275 & & 3 \\
\hline .18 & 96284 & 96289 & 96294 & 96298 & 963 & 96 & 96 & 96 & 96322 & 27 & 3 \\
\hline .19 & 96332 & 96336 & $9634 \mathrm{I}$ & 96346 & 963 & 96355 & 96360 & 96365 & 96369 & 74 & 4 \\
\hline 9.20 & .96 & .96384 & 9 & .9 & & .96 & & & & & 5 \\
\hline .21 & 964 & 964 & 964 & 96 & 96 & 96 & & & & & I \\
\hline .22 & 96473 & 96478 & 96483 & 96487 & 964 & 96 & 9 & 06 & 965 I I & 96 & I \\
\hline .23 & 96520 & 96525 & 96530 & 96534 & 965 & 965 & 96548 & 96553 & $9655^{8}$ & 96 & 2 \\
\hline .24 & 96567 & 96572 & 96577 & $9^{6} 5^{8 I}$ & $965^{86}$ & 96591 & 96595 & 96600 & 96605 & 96609 & 2 \\
\hline 9.25 & .96614 & .96619 & .96624 & .96628 & .96633 & .96638 & .96642 & .96647 & .96652 & .96656 & 3 \\
\hline .26 & $9666 \mathrm{r}$ & 96666 & 966 & 96675 & 96 & 96685 & 96689 & & 96699 & 96 & 3 \\
\hline .27 & 96708 & 96713 & 96717 & 96722 & 96727 & $9673 \mathrm{I}$ & 96736 & $9674 \mathrm{I}$ & 96745 & & 4 \\
\hline .28 & 96755 & 96759 & 96764 & 96769 & 96774 & 96778 & 96783 & 96788 & 96792 & 96797 & 4 \\
\hline 29 & 96802 & 96806 & 96811 & 96816 & 96820 & 96825 & 96830 & 96834 & 96839 & 96 & 5 \\
\hline 9.30 & .96848 & .96853 & .96858 & .96862 & .96867 & .96872 & .968 & 81 & .9 & .9 & 4 \\
\hline & 96895 & 96900 & 96904 & 969 & $96 \mathrm{c}$ & $96 \mathrm{c}$ & 96 & 96 & 96 & & 0 \\
\hline .32 & 96942 & 96946 & 9695 I & 96956 & 96960 & 96965 & 96 & 96 & 96979 & & I \\
\hline 33 & 96988 & 96993 & 96997 & 97002 & 97007 & 97 OI I & 97 & & 97 & & I \\
\hline .34 & $97 \bigcirc 35$ & $97 \bigcirc 39$ & 97044 & 97049 & $97 \bigcirc 53$ & 97058 & 97063 & 97067 & 97072 & 97077 & 2 \\
\hline 9.35 & .97081 & .97086 & .97090 & .97 & .9 & .97104 & 09 & 14 & .97118 & 23 & 2 \\
\hline & 97128 & $97 \times 32$ & $97 \times 37$ & 97 & $97 \times 46$ & $5 \mathrm{I}$ & & 60 & & & 2 \\
\hline .37 & 971 & 97179 & $97 \mathbf{1} 83$ & 97188 & 97192 & 97197 & 97 & 97206 & 97 & 9 & 3 \\
\hline & 97220 & 97225 & 972 & 97234 & 972 & 97243 & 97248 & 97253 & 97257 & & 3 \\
\hline 30 & & & & & & & & & 97304 & & 4 \\
\hline 9.40 & .97313 & 7 & .97 & .97 & .97 & 336 & & & & & 5 \\
\hline .41 & 97359 & 97364 & 97368 & 97373 & 973 & 97382 & $973^{8} 7$ & 9739 I & 97396 & & I \\
\hline .42 & 97405 & 97410 & 97414 & 97419 & 974 & 97428 & 97433 & 97 & & & I \\
\hline .43 & $9745^{I}$ & $9745^{6}$ & 97460 & 97465 & 974 & 97474 & 974 & & & & 2 \\
\hline .44 & 97497 & 97502 & 97506 & 97511 & 97516 & 97520 & 97525 & 97529 & 97534 & 97539 & 2 \\
\hline 9.45 & .97543 & .97548 & .97552 & .975 & .97562 & .97566 & $.9757 x$ & .97575 & $.975^{80}$ & $.975^{85}$ & 3 \\
\hline & 97589 & 97594 & 97598 & 97603 & 97607 & 97612 & 97617 & & 97626 & & 3 \\
\hline .47 & 97635 & 97640 & 97644 & 97649 & 97653 & 97658 & 97663 & 97667 & 97672 & 97676 & 4 \\
\hline .48 & 97681 & 97685 & 97690 & 97695 & 97699 & 97704 & 97708 & 97713 & & & 4 \\
\hline .49 & 97727 & 9773 I & 97736 & 97740 & 97745 & 97749 & 97754 & 97759 & 97763 & 97768 & 5 \\
\hline
\end{tabular}




\begin{tabular}{|c|c|c|c|c|c|c|c|c|c|c|c|}
\hline No. & 0 & I & 2 & 3 & 4 & 5 & 6 & 7 & 8 & 9 & $\mid \begin{array}{l}\mid \text { INTP } \\
\text { TAB. }\end{array}$ \\
\hline 9.50 & .97772 & .97777 & $.977^{82}$ & & .97791 & .97795 & .97800 & $.97 \mathrm{Sa}_{4}$. & .97809. & $.978_{13}$ & 4 \\
\hline .51 & 97818 & $97 \$ 23$ & 97827 & $97 \$_{32}$ & $97 S_{36}$ & $97 S_{41}$ & $978_{45}$ & $97 S_{50}$ & $97 \delta_{55}$ & $97 S_{59}$ & o \\
\hline .52 & $97 \mathrm{S6}_{4}$ & 97868 & 97873 & $97 S_{77}$ & $97 \mathrm{SB}_{2}$ & 97886 & 97891 & 97 S96 & 97900 & 97905 & 1 \\
\hline .53 & 97009 & 97914 & 97918 & 97923 & 97928 & 97932 & 97937 & $9794^{1}$ & $979+6$ & $9795^{\circ}$ & I \\
\hline .54 & 97955 & 97959 & 97964 & 97968 & 97973 & $9797^{8}$ & 97982 & 97987 & 97991 & 97996 & 2 \\
\hline 9.55 & .98000 & $.9 \$ 005$ & .98009 & .98014 & .98019 & .98023 & .98028 & $.9 \mathrm{SO}_{32}$. & .98037 & .98041 & 2 \\
\hline .56 & 95046 & $99^{S} \circ 50$ & $9 \$ 055$ & 98059 & 93064 & 99068 & 98073 & 98078 & 98082 & 98087 & 2 \\
\hline .57 & $9 \$ \circ 91$ & $9 \$ 096$ & $9 \$ 100$ & 98 105 & $9 \$ 109$ & yS 114 & 98118 & 98123 & 98127 & $9813^{2}$ & 3 \\
\hline $.5^{\mathrm{S}}$ & $9 \$ 137$ & 98141 & 98146 & $98 \times 50$ & $9 S 155$ & 98159 & 98164 & $9 \$ 168$ & 98173 & 98177 & 3 \\
\hline .59 & 98182 & 98 IS6 & $9 S 191$ & 98195 & 98200 & 98204 & 98209 & 98214 & 98218 & 98223 & 4 \\
\hline 9.60 & .98227 & $.9823^{2}$ & .98236 & $.9824 \mathrm{I}$ & .98245 & .98250 & .98254 & .98259 & .98263 & .98268 & 5 \\
\hline $.6 t$ & $9827^{2}$ & $98=77$ & 98281 & $98 \geq 86$ & 93290 & $9 \$ 295$ & 98299 & 98304 & 98308 & $9^{8} 3^{1} 3$ & I \\
\hline .62 & $9^{8} 3^{18}$ & 98322 & 98327 & 98331 & 98336 & 98340 & $9^{8} 345$ & 98349 & 98354 & $9^{8} 35^{8}$ & I \\
\hline .63 & 98363 & $9^{8} 3^{67}$ & $9^{8} 37^{2}$ & $9^{9} 37^{6}$ & 98381 & $9 S_{3} 85$ & 98390 & 98394 & 98399 & 98403 & 2 \\
\hline .64 & 93408 & $9^{8}+12$ & 98417 & $9 S_{421}$ & 98426 & 98430 & $9^{8} 435$ & 98439 & $9^{8} 444$ & $9844^{8}$ & 2 \\
\hline 9.65 & $.9^{8}+53$. & $.9^{8} 457$ & .98462 & $.98+66$ & .98471 & .98475 & .98480 & $.984_{4}{ }_{4}$ & .98489 & .98493 & 3 \\
\hline .66 & $9 S 49 S$ & 98502 & 98507 & 98511 & $98 ; 16$ & 98520 & 98525 & 98529 & $9^{95} 534$ & $9^{S} 53^{8}$ & 3 \\
\hline .67 & 98543 & 98547 & $9855^{2}$ & $9 \$ 556$ & 9856 I & 98565 & $9^{8} 57^{\circ}$ & 98574 & $3^{8} 579$ & $9^{\mathrm{S}} 5_{5} \mathrm{~s}_{3}$ & 4 \\
\hline .68 & $98 ; 88$ & 98592 & 98597 & 98601 & 98605 & 98010 & 98614 & 98619 & 98623 & 98628 & 4 \\
\hline .69 & 98632 & 98637 & $9864 I$ & 98646 & 98650 & 98655 & 98659 & 98664 & 98668 & 98673 & 5 \\
\hline 9.70 & .98677. & .98682 & .08686 & $.9869 \mathrm{I}$ & .98695 & .98700 & .98704 & $.9 \$ 709$ & $.987^{8} 3$ & .98717 & 4 \\
\hline .71 & 98722 & 98726 & 98731 & $9 \$ 735$ & 98740 & 98744 & 98749 & 98753 & $9875^{8}$ & $98-62$ & 0 \\
\hline .72 & 98767 & 98771 & 98776 & 98750 & 98784 & 98789 & 98793 & 98798 & $98 \mathrm{So2}$ & 98807 & I \\
\hline .73 & $98 \mathrm{sin}$ & 98816 & $9 \mathrm{~S} S 20$ & $98 \$ 25$ & 98829 & 98834 & $9883^{8}$ & 98843 & 98847 & 98851 & 1 \\
\hline .74 & 98856 & 98860 & $98 \$ 65$ & 98869 & 98874 & 98878 & $9888_{3}$ & 98887 & 98892 & 98596 & 2 \\
\hline 9.75 & $.9^{8}$ yoo. & $.9 \$ 905$ & .98909 & $.98,914$. & .98918 & .98923 & .98927 & .98932 & .98936 . & .98941 & 2 \\
\hline .76 & 98945 & $9^{\$} 949$ & 93954 & ys & $9^{8} 963$ & 7 & 72 & 6 & 1 & 85 & 2 \\
\hline .77 & 989.99 & 98994 & 98998 & 99003 & 99007 & 99012 & 99016 & 99021 & 99025 & 99029 & 3 \\
\hline .78 & 99034 & $993^{8}$ & $990+3$ & 99047 & 99052 & 99056 & 99061 & 99065 & 99069 & 99074 & 3 \\
\hline .79 & $9907^{8}$ & $9908_{3}$ & $990 \$ 7$ & 99092 & 99096 & 99100 & 99105 & 99109 & 99 เ14 & 99118 & 4 \\
\hline 9.80 & .99123 & .99127 & .99131 & .99136 & .99140 & .99145 & .99149 & .99154 . & .99158. & .99162 & 5 \\
\hline$S t$ & 99167 & 99 וז & 99176 & 99180 & $9918_{5}$ & 99189 & 99193 & 99108 & 99202 & 99207 & 1 \\
\hline .82 & 99211 & 99216 & 99220 & 9) 224 & 99229 & 99233 & $9923^{8}$ & 99242 & 99247 & 99251 & I \\
\hline .83 & 9) 255 & 99260 & 99264 & $992(x)$ & 99273 & 99277 & 99282 & 99286 & 99291 & 99295 & 2 \\
\hline .84 & 99300 & 99304 & 99308 & $993^{1} 3$ & 99317 & 99322 & 99326 & 99330 & 99335 & 99339 & 2 \\
\hline 9.85 & .99344 & .99 $34^{8}$ & .99352 & .99357 & $.993^{61}$ & .99366 & .99370 & .99374 . & .99379 & $.993^{8} 3$ & 3 \\
\hline .86 & 99388 & 99) 392 & 99396 & 99401 & 99405 & 99410 & $99+14$ & 90419 & 99423 & 99427 & 3 \\
\hline .87 & $9943^{2}$ & $99+36$ & $9944^{1}$ & 99445 & 99449 & 99454 & 99458 & 99463 & 99467 & 99471 & 4 \\
\hline .88 & $9947^{6}$ & 994 So & $994^{8} 4$ & $994^{89}$ & 99493 & 99498 & 99502 & 99506 & 99511 & 99515 & 4 \\
\hline .89 & 99520 & 99524 & $995^{28}$ & 99 5.33 & 99537 & 99542 & $9954^{6}$ & $9955^{\circ}$ & 99555 & 99559 & 5 \\
\hline 9.90 & $.995^{64}$ & .99 568 & .99 572 & .99577 & $.995^{81}$ & $.905^{85}$ & .99590 & .90594 & .99509 & .99603 & 4 \\
\hline .91 & 99607 & 99612 & 99616 & 99621 & 99625 & 99629 & 90634 & $996_{3} \mathrm{~s}$ & 99642 & 99647 & 0 \\
\hline .92 & 99651 & 99656 & 99660 & 99664 & 99669 & 99673 & 99677 & $996 \mathbb{S}_{2}$ & 99686 & $99(x) 1$ & 1 \\
\hline .93 & $y y^{(x) 5}$ & $99(x) 9$ & 90704 & 99708 & 99712 & 99717 & 90721 & 99726 & 99730 & 99734 & 1 \\
\hline .94 & 99739 & 9974.3 & 90747 & 99752 & $9975^{6}$ & 99760 & $997^{6} 5$ & $997^{60}$ & 99774 & $9977^{8}$ & 2 \\
\hline 0.05 & $997^{82}$ & $.997^{8} 7$ & 40701 & .09795 & .99 \$oo & .09804 & .90808 & $.99 \mathrm{~S}_{3}$ & .99817. & .99822 & 2 \\
\hline 90 & 90826 & 90830 & $99 \$_{35}$ & 99839 & $99 \mathrm{~S}_{43}$ & 99848 & 99852 & $998_{56}$ & 96864 & 90865 & 2 \\
\hline .197 & $998-6$ & 99874 & 99875 & $99 \mathrm{Ss}_{3}$ & $90 \mathrm{SS7}$ & $90 \mathrm{~S} 91$ & 99 So6 & 90900 & 9990.4 & 99909 & 3 \\
\hline 498 & 99913 & 99017 & 99022 & 99926 & 99930 & 99035 & 99930 & 90944 & $9994 \mathrm{~S}$ & 99952 & 3 \\
\hline .90 & 90457 & 90061 & (x) 965 & 99970 & 90974 & $9947^{8}$ & $99^{\$} 3$ & 99087 & 99991 & 99996 & 4 \\
\hline
\end{tabular}




\section{SQUARE ROOTS AND SQUARES.}

Note. The table gives roots directly, squares by inverse interpolation.

\begin{tabular}{|c|c|c|c|c|c|c|c|c|c|c|c|c|}
\hline 10. & 0 & I & 2 & 3 & 4 & 5 & 6 & 7 & 8 & 9 & \multicolumn{2}{|c|}{$\begin{array}{l}\text { Interpola, } \\
\text { for Thousandths. }\end{array}$} \\
\hline 1.0 & 1.000 & 1.005 & 1.010 & 1.015 & 1.020 & 1.025 & 1.030 & 1.034 & 1.039 & 1.044 & 5 & 4 \\
\hline .I & 1.049 & 1.054 & $1.05^{8}$ & 1.063 & 1.068 & 1.072 & I.077 & I. 082 & I.086 & 1.091 & I & 0 \\
\hline .2 & I.095 & 1.100 & I. 105 & I.109 & I.II4 & 1.118 & I.1 22 & 1.127 & I.I 3I & I.1 36 & I & I \\
\hline$\cdot 3$ & I.I 40 & I.145 & I.I 49 & I.153 & I.1 58 & 1.162 & I.I 66 & 1.170 & I.1 75 & I.I 79 & 2 & I \\
\hline .4 & 1.183 & 1.187 & 1.192 & 1.196 & 1.200 & 1.204 & I. 208 & 1.212 & I. 217 & I.22I & 2 & 2 \\
\hline 1.5 & 1.225 & I. 229 & 1.233 & 1.237 & I.24I & 1.245 & I. 249 & 1.253 & I.257 & $\mathrm{I} .26 \mathrm{I}$ & 3 & 2 \\
\hline .6 & I. 265 & I. 269 & I. 273 & I. 277 & $\mathrm{I} .28 \mathrm{I}$ & 1.285 & I. 288 & 1.292 & I.296 & 1.300 & 3 & 2 \\
\hline .7 & I. 304 & I. 308 & 1.311 & I. 315 & 1.319 & 1.323 & 1.327 & 1.330 & I. 334 & I. $33^{8}$ & 4 & 3 \\
\hline .8 & I. 342 & 1.345 & I. 349 & 1. 353 & I. 356 & 1.360 & I. 364 & 1.367 & I. 371 & I. 375 & 4 & 3 \\
\hline .9 & I. $37^{8}$ & $1.3^{82}$ & I. 386 & I. 389 & I.393 & I.396 & 1.400 & 1.404 & 1.407 & I.4II & 5 & 4 \\
\hline 2.0 & 1.414 & 1.418 & 1.421 & I. 425 & 1.428 & 1.4 & I. 435 & I.439 & 1.442 & 1.446 & 4 & 3 \\
\hline . I & I. 449 & I. 453 & I. 456 & I. 459 & 1.463 & 1.466 & 1.470 & I. 473 & 1.476 & 1.480 & o & 0 \\
\hline .2 & 1.483 & I. 487 & 1.490 & 1.493 & I.497 & 1.500 & 1.503 & I.507 & $1.5^{10}$ & $1.5^{13}$ & I & I \\
\hline .3 & 1.517 & I. 520 & I. 523 & $1.526^{\circ}$ & x. 530 & 1.533 & 1. 536 & I. 539 & I. 543 & I. 546 & I & I \\
\hline .4 & I.549 & I. $55^{2}$ & $1.55^{6}$ & 1.559 & 1. 562 & 1.565 & I. 568 & 1.572 & I. 575 & I. $57^{8}$ & 2 & I \\
\hline 2.5 & I. $5^{81}$ I & I. $5^{84}$ & I. $5^{87}$ & I. $59 \mathrm{I}$ & I. 594 & I.597 & 1.600 & I. 603 & 1.606 & 1.609 & 2 & 2 \\
\hline .6 & 1.612 & I. 616 & I. 619 & 1.622 & 1.625 & 1. 628 & 1.631 & I. 634 & 1.637 & I. 640 & 2 & 2 \\
\hline .7 & 1.643 & I. 646 & 1.649 & $1.65^{2}$ & 1. 655 & 1.658 & I. $66 \mathrm{I}$ & 1.664 & $x .667$ & I. $67^{\circ}$ & 3 & 2 \\
\hline .8 & I. 673 & I. 676 & x.679 & 1.682 & 1.685 & 1.688 & I.69I & 1.694 & 1.697 & 1.700 & 3 & 2 \\
\hline .9 & 1.703 & I.706 & 1.709 & 1.712 & 1.715 & $x .718$ & 1.720 & 1.723 & 1.726 & 1.729 & 4 & 3 \\
\hline 3.0 & 1.732 & I. 735 & $1.73^{8}$ & I. 741 & I. 744 & 1. 746 & I. 749 & I. $75^{2}$ & I. 755 & $1.75^{8}$ & 3 & 2 \\
\hline .I & 1.761 & I. 764 & 1.766 & 1.769 & 1.772 & I. 775 & 1. 778 & 1.780 & 1.783 & I.786 & 0 & o \\
\hline .2 & 1. 789 & I. 792 & 1.794 & I.797 & 1.800 & 1.803 & I.806 & 1.808 & $\mathrm{I} .8 \mathrm{II}$ & 1.814 & I & o \\
\hline$\cdot 3$ & 1.817 & I.819 & 1.822 & 1.825 & I. 828 & 1.830 & 1.833 & 1. 836 & I. $83^{8}$ & I. $84 \mathrm{I}$ & I & I \\
\hline .4 & 1.844 & 1.847 & I. 849 & $1.85^{2}$ & I. 855 & 1.857 & 1.860 & 1.863 & I. 865 & I. 868 & I & I \\
\hline 3.5 & I. $87 \mathrm{I}$ & I. 873 & 1. 876 & I. 879 & I. $88 \mathrm{I}$ & I. 884 & 1.887 & I. 889 & I. 892 & I. 895 & 2 & $\mathbf{I}$ \\
\hline .6 & 1.897 & 1.900 & 1.903 & I.905 & 1.908 & 1.910 & 1.913 & 1.916 & I.91 8 & 1.921 & 2 & I \\
\hline$\cdot 7$ & 1.924 & 1.926 & 1.929 & 1.931 & I.934 & 1.936 & I.939 & 1.942 & I. 944 & 1.947 & 2 & I \\
\hline .8 & I. 949 & 1.952 & 1.954 & 1.957 & 1.960 & 1.962 & 1.965 & 1.967 & 1.970 & 1.972 & 2 & 2 \\
\hline .9 & I.975 & 1.977 & I. 980 & 1.982 & 1.985 & 1.987 & I.990 & I.992 & I.995 & x.997 & 3 & 2 \\
\hline 4.0 & 2.000 & 2.002 & 2.005 & 2.007 & 2.010 & 2.012 & 2.015 & 2.017 & 2.020 & 2.022 & 8 & 2 \\
\hline .1 & 2.025 & 2.027 & 2.030 & 2.032 & 2.035 & 2.037 & 2.040 & 2.042 & 2.045 & 2.047 & 0 & o \\
\hline .2 & 2.049 & $2.05^{2}$ & 2.054 & 2.057 & 2.059 & 2.062 & 2.064 & 2.066 & 2.069 & $2.07 \mathrm{I}$ & I & 0 \\
\hline .3 & 2.074 & 2.076 & 2.078 & 2.081 & 2.083 & 2.086 & 2.088 & 2.090 & 2.093 & 2.095 & I & I \\
\hline .4 & 2.098 & 2.100 & 2.102 & 2.105 & 2.107 & 2.110 & 2.112 & 2.114 & 2.117 & 2.119 & I & I \\
\hline 4.5 & 2.121 & 2.124 & 2.126 & 2.128 & $2.13 \mathrm{I}$ & 2.133 & 2.135 & $2.13^{8}$ & 2.140 & 2.142 & 2 & $\mathbf{I}$ \\
\hline .6 & 2.145 & 2.147 & 2.149 & 2.152 & 2.154 & 2.156 & 2.159 & $2.16 I$ & 2.163 & 2.166 & 2 & I \\
\hline .7 & 2.168 & 2.170 & 2.173 & 2.175 & 2.177 & 2.179 & 2.182 & 2.184 & 2.186 & 2.189 & 2 & I \\
\hline .8 & 2.191 & 2.193 & 2.195 & 2.198 & 2.200 & 2.202 & 2.205 & 2.207 & 2.209 & 2.211 & 2 & 2 \\
\hline .9 & 2.214 & 2.216 & 2.218 & 2.220 & 2.223 & 2.225 & 2.227 & 2.229 & 2.232 & 2.234 & 3 & 2 \\
\hline
\end{tabular}


SQ. RTS. \& SQRS.

\begin{tabular}{|c|c|c|c|c|c|c|c|c|c|c|c|c|}
\hline No. & 0 & I & 2 & 3 & 4 & 5 & 6 & 7 & 8 & 9 & \multicolumn{2}{|c|}{$\begin{array}{l}\text { Interpola. for } \\
\text { Thousandths. }\end{array}$} \\
\hline 5.0 & 2.236 & $2.23^{8}$ & 2.241 & 2.243 & 2.245 & 2.247 & 2.249 & 2.252 & 2.254 & 2.256 & 3 & 2 \\
\hline .1 & $2.25^{8}$ & 2.261 & 2.263 & 2.265 & 2.267 & 2.269 & 2.272 & 2.274 & 2.276 & 2.278 & 0 & 0 \\
\hline .2 & $2.2 \mathrm{So}$ & $2.2 S_{3}$ & 2.285 & $2.2 S_{7}$ & 2.289 & $2.29 \mathrm{I}$ & 2.293 & 2.296 & 2.298 & 2.300 & I & 0 \\
\hline$\cdot 3$ & 2. 302 & 2. 304 & 2.307 & 2.309 & 2.311 & $2.3^{13}$ & 2.315 & 2.317 & 2.319 & 2.322 & I & I \\
\hline .4 & 2.324 & 2.326 & 2.328 & 2.330 & 2.332 & 2.335 & 2.337 & 2.339 & 2.341 & 2.343 & I & 1 \\
\hline $5 \cdot 5$ & 2.345 & 2.347 & 2.349 & $2.35^{2}$ & 2.354 & $2.35^{6}$ & $2.35^{8}$ & 2.360 & 2.362 & 2.364 & 2 & I \\
\hline .6 & 2. 366 & 2.369 & 2.371 & 2.373 & 2.375 & 2.377 & 2.379 & $2.3^{81}$ & 2. $3^{8} 3$ & 2. 385 & 2 & I \\
\hline .7 & 2. $3^{8} 7$ & 2.390 & 2.392 & 2.394 & 2. 396 & 2.398 & 2.400 & 2.402 & 2.404 & 2.406 & 2 & I \\
\hline .8 & 2.408 & 2.410 & 2.412 & 2.415 & 2.417 & 2.419 & 2.421 & 2.423 & 2.425 & 2.427 & 2 & 2 \\
\hline .9 & 2.429 & $2.43^{1}$ & 2.433 & 2.435 & 2.437 & 2.439 & $2.44 \mathrm{I}$ & 2.443 & 2.445 & 2.447 & 3 & 2 \\
\hline 6.0 & 2.449 & 2.452 & 2.454 & $2.45^{6}$ & $2.45^{8}$ & 2.460 & 2.462 & 2.464 & 2.466 & 2.468 & 8 & 8 \\
\hline . I & 2.470 & 2.472 & 2.474 & 2.476 & $2.47^{8}$ & $2.4 \mathrm{SO}$ & 2.482 & 2.484 & 2.486 & 2.488 & 0 & 0 \\
\hline .2 & 2.490 & 2.492 & 2.494 & 2.496 & $2.49^{8}$ & 2.500 & 2.502 & 2.504 & 2.506 & 2.508 & 1 & 0 \\
\hline$\cdot 3$ & 2.510 & 2.512 & 2.514 & 2.516 & 2.518 & 2.520 & 2.522 & 2.524 & 2.526 & 2.528 & 1 & $I$ \\
\hline 4 & 2.530 & 2.532 & 2.534 & 2.536 & $2.53^{8}$ & 2. 540 & $2.54^{2}$ & 2.544 & 2.546 & 2.548 & 1 & 1 \\
\hline 6.5 & 2.550 & $2.55^{I}$ & 2.553 & 2.555 & 2.557 & 2.559 & $2.56 \mathrm{I}$ & 2.563 & 2.565 & 2.567 & 2 & I \\
\hline .6 & 2.560 & 2.571 & 2.573 & 2.575 & 2.577 & 2.579 & $2.58 \mathrm{I}$ & $2.5^{8} 3$ & 2.585 & 2.587 & 2 & 1 \\
\hline .7 & 2.588 & 2.590 & 2.592 & 2.594 & 2.596 & 2.598 & 2.600 & 2.602 & 2.604 & 2.606 & 2 & I \\
\hline .8 & 2.608 & 2.610 & 2.612 & $2.61_{3}$ & 2.615 & 2.617 & 2.619 & 2.621 & 2.623 & 2.625 & 2 & 2 \\
\hline .9 & 2.627 & 2.629 & 2.631 & 2.632 & 2.634 & 2.636 & 2.638 & 2.640 & 2.642 & $2.6+4$ & 3 & 2 \\
\hline 7.0 & 2.646 & 2.648 & 2.650 & 2.651 & 2.653 & 2.655 & 2.657 & 2.659 & $2.66 \mathrm{I}$ & $2.66_{3}$ & 2 & 1 \\
\hline .I & 2.665 & 2.666 & 2.668 & 2.670 & 2.672 & 2.674 & 2.676 & 2.678 & 2.680 & $2.68 I$ & 0 & 0 \\
\hline .2 & 2.683 & 2.685 & 2.687 & 2.689 & $2.69 \mathrm{I}$ & 2.693 & 2.694 & 2.696 & 2.698 & 2.700 & o & 0 \\
\hline$\cdot 3$ & 2.702 & 2.704 & 2.706 & 2.707 & 2.709 & 2.711 & 2.713 & 2.715 & 2.717 & 2.718 & 1 & 0 \\
\hline .4 & 2.720 & 2.722 & 2.724 & 2.726 & 2.728 & 2.729 & $2.73 \mathrm{I}$ & 2.733 & 2.735 & 2.737 & 1 & 0 \\
\hline 7.5 & 2.739 & 2.740 & 2.742 & 2.744 & 2.746 & 2.748 & 2.750 & $2.75^{1}$ & 2.753 & 2.755 & 1 & 1 \\
\hline .6 & 2.757 & 2.759 & 2.760 & 2.762 & 2.764 & 2.766 & 2.768 & 2.769 & 2.771 & 2.773 & 1 & 1 \\
\hline 7 & 2.775 & 2.777 & 2.778 & $2.7^{80}$ & 2. $7^{82}$ & $2.7^{8} 4$ & 2.786 & $2.7 \delta_{7}$ & 2.789 & 2.791 & I & I \\
\hline .8 & 2.793 & 2.795 & 2.796 & 2. 798 & 2.800 & $2 . \mathrm{SO}_{2}$ & 2.804 & 2.805 & 2.807 & 2.509 & 2 & I \\
\hline .9 & 2.811 & 2.812 & 2.814 & 2.816 & $2.81 \mathrm{~S}$ & 2.820 & 2.821 & 2.823 & 2.825 & 2.827 & 2 & 1 \\
\hline 8.0 & 2.828 & 2.830 & 2.832 & 2.834 & 2.835 & 2.837 & 2.839 & $2.84 \mathrm{I}$ & 2.843 & 2.844 & 2 & 1 \\
\hline .1 & 2.846 & 2.848 & $2.5_{50}$ & $2.85 \mathrm{I}$ & 2.853 & 2.855 & 2.857 & 2.858 & 2.860 & 2.862 & 0 & 0 \\
\hline .2 & 2.864 & 2.865 & 2.867 & 2.869 & 2.871 & 2.872 & 2.874 & 2.876 & 2.877 & 2.879 & 0 & 0 \\
\hline$\cdot 3$ & 2.881 & $2.88_{3}$ & 2.884 & 2.886 & 2.888 & 2.890 & $2.89 \mathrm{I}$ & 2.893 & 2.895 & 2.897 & I & 0 \\
\hline 4 & 2.898 & 2.900 & 2.902 & 2.903 & 2.905 & 2.907 & 2.909 & 2.910 & 2.912 & 2.914 & 1 & 0 \\
\hline 8.5 & 2.915 & 2.917 & 2.9)19 & 2.921 & 2.922 & 2.924 & 2.926 & 2.927 & 2.929 & 2.931 & 1 & 1 \\
\hline .6 & 2.933 & 2.934 & 2.936 & 2.9) 38 & 2.9 .39 & 2.941 & 2.943 & 2.944 & 2.946 & 2.948 & 1 & 1 \\
\hline .7 & 2.950 & 2.951 & 2.95 .3 & 2.955 & $2.95^{6}$ & $2.95^{8}$ & 2.960 & 2.961 & 2.963 & 2.965 & I & 1 \\
\hline .8 & 2.966 & 2.968 & 2.970 & 2.972 & 2.973 & 2.975 & 2.977 & $2.97^{\mathrm{S}}$ & 2.980 & $2.9 \mathrm{~S}_{2}$ & 2 & 1 \\
\hline 9 & $2.98_{3}$ & $2.98_{5}$ & 2.987 & 2.988 & 2.990 & 2.992 & 2.993 & 2.995 & 2.997 & 2.998 & 2 & $I$ \\
\hline 9.0 & 3.000 & 3.002 & 3.003 & 3.005 & 3.007 & 3.008 & 3.010 & 3.012 & 3.013 & 3.015 & 2 & 1 \\
\hline .1 & 3.017 & 3.018 & 3.020 & 3.022 & 3.023 & 3.025 & 3.027 & 3.028 & 3030 & 3.032 & 0 & 0 \\
\hline .2 & 3.033 & 3.035 & 3.036 & $3.03^{8}$ & 3.040 & 3.041 & 3.043 & 3.045 & 3.046 & 3.048 & 0 & 0 \\
\hline .3 & 3.050 & 3.051 & 3.053 & 3.055 & 3.056 & 3.058 & 3.059 & 3.061 & 3.063 & $3.06_{4}$ & 1 & 0 \\
\hline 4 & 3.066 & 3.068 & 3.060 & 3.071 & 3.072 & 3.074 & 3.076 & 3.077 & 3.079 & 3.081 & I & 0 \\
\hline 9.5 & 3.082 & $305_{4}$ & $3.0 \$_{5}$ & $3.08_{7}$ & 3.089 & 3.090 & $3 \cdot(x) 2$ & 3.094 & 3.095 & 3.097 & 1 & 1 \\
\hline .6 & $3 .(0) 8$ & 3.100 & 3.102 & $3 \cdot 10_{3}$ & 3.105 & 3.106 & 3.108 & 3.180 & 3.111 & 3.113 & 8 & I \\
\hline .7 & 3.114 & 3116 & 3.115 & 3.119 & 3.121 & 3.122 & 3.124 & 3.126 & 3.127 & 3.129 & I & $I$ \\
\hline .8 & 3130 & 3.132 & 3.134 & 3.135 & 3.137 & $3.13^{8}$ & 3.140 & 3.142 & 3.143 & 3.845 & 2 & $t$ \\
\hline .9 & 3.146 & 3.148 & 3.150 & 3.151 & 3.153 & 3.154 & 3.156 & $3.15^{\mathrm{S}}$ & 3.150 & 3.161 & 2 & 1 \\
\hline
\end{tabular}




\section{SQUARE ROOTS AND SQUARES.}

\begin{tabular}{|c|c|c|c|c|c|c|c|c|c|c|c|c|}
\hline No. & 0 & I & 2 & 3 & 4 & 5 & 6 & 7 & 8 & 9 & \multicolumn{2}{|c|}{$\begin{array}{l}\text { Interpola, } \\
\text { for Hundredths. }\end{array}$} \\
\hline 10. & 3.162 & $3.17^{8}$ & 3.194 & 3.209 & 3.225 & 3.240 & 3.256 & 3.271 & 3.286 & $3 \cdot 302$ & 16 & 14 \\
\hline II. & 3.317 & $3.33^{2}$ & 3.347 & $3 \cdot 362$ & $3 \cdot 37^{6}$ & 3.391 & 3.406 & $3 \cdot 421$ & 3.435 & 3.450 & 2 & I \\
\hline 12. & 3.464 & 3.479 & 3.493 & 3.507 & 3.521 & 3.536 & $3.55^{\circ}$ & $3 \cdot 5^{64}$ & $3.57^{8}$ & 3.592 & 3 & 3 \\
\hline 13. & 3.606 & 3.619 & 3.633 & 3.647 & $3.66 \mathrm{I}$ & 3.674 & 3.688 & 3.701 & 3.715 & 3.7 & 5 & 4 \\
\hline 14. & 3.742 & 3.755 & 3.768 & 3.782 & 3.795 & 3.808 & $3.82 \mathrm{I}$ & 3.834 & 3.847 & 3.860 & 6 & 6 \\
\hline 15. & 3.873 & 3.886 & 3.899 & 3.912 & 3.924 & 3.937 & 3.950 & 3.962 & 3.975 & 3.987 & 8 & 7 \\
\hline 16. & 4.000 & 4.012 & 4.025 & 4.037 & 4. & 2 & 4.074 & 4.087 & 4.099 & 4. III & 10 & 8 \\
\hline 17. & 4.123 & 4.135 & 4.147 & 4.159 & 4.171 & 4.183 & 4.195 & 4.207 & 4.219 & 4.231 & II & 10 \\
\hline 18. & 4.243 & 4.254 & 4.266 & 4.278 & 4.290 & 4.301 & $4 \cdot 3^{1} 3$ & $4 \cdot 324$ & $4 \cdot 336$ & $4 \cdot 347$ & 13 & II \\
\hline 19. & $4 \cdot 359$ & 4.370 & $4 \cdot 3^{82}$ & $4 \cdot 393$ & 4.405 & 4.416 & 4.427 & $4.43^{8}$ & 4.450 & 4.461 & 14 & 13 \\
\hline 20. & 4.472 & 4.483 & 4.494 & 4.506 & $4 \cdot 5^{17}$ & $4 \cdot 528$ & 4.539 & $4.55^{\circ}$ & $4 \cdot 5^{6} \mathrm{I}$ & 4.572 & 12 & 10 \\
\hline 21. & $4.5^{8} 3$ & 4.593 & 4.604 & 4.615 & 4.6 & 4.637 & 4.648 & 4.658 & 4.669 & 4.680 & I & I \\
\hline 22. & 4.690 & $4.70 \mathrm{I}$ & 4.712 & 4.722 & 4.733 & 4.743 & 4.754 & $4 \cdot 764$ & 4.775 & $4 \cdot 78_{5}$ & 2 & 2 \\
\hline 23. & 4.796 & 4.806 & 4.817 & 4.827 & 4.837 & 4.848 & $4.85^{8}$ & 4.868 & 4.879 & 4.889 & 4 & 3 \\
\hline 24. & 4.899 & 4.909 & 4.919 & 4.930 & 4.940 & $4.95^{\circ}$ & 4.960 & 4.970 & 4.980 & 4.990 & 5 & 4 \\
\hline 25. & 5.000 & 5.010 & 5.020 & 5.030 & 5.040 & 5.050 & 5.060 & 5.070 & 5.079 & 5.089 & 6 & 5 \\
\hline 26. & 5.099 & 5.109 & 5.119 & 5.128 & $5.13^{8}$ & 5.148 & $5.15^{8}$ & 5.167 & 5.177 & 5.187 & 7 & 0 \\
\hline 27. & 5.196 & 5.206 & 5.215 & 5.225 & 5.235 & 5.244 & 5.254 & 5.263 & 5.273 & 5.282 & 8 & 7 \\
\hline 28. & 5.292 & $5 \cdot 30 \mathrm{I}$ & $5 \cdot 310$ & $5 \cdot 320$ & $5 \cdot 329$ & $5 \cdot 339$ & $5 \cdot 34^{8}$ & $5 \cdot 357$ & $5 \cdot 367$ & $5 \cdot 376$ & 10 & 8 \\
\hline 29. & $5 \cdot 385$ & 5.394 & 5.404 & 5.413 & $5 \cdot 422$ & $5.43 \mathrm{I}$ & $5 \cdot 44 \mathrm{I}$ & 5.450 & 5.459 & $5 \cdot 468$ & II & 9 \\
\hline 30. & 5.477 & 5.486 & 5.495 & 5.505 & 5.5 & 3 & & & & & 9 & 8 \\
\hline 31. & 5.568 & 5.577 & $5 \cdot 5^{86}$ & 5.595 & 5.6 & 2 & I & 0 & 39 & 8 & I & I \\
\hline 32. & 5.657 & 5.666 & 5.675 & 5.683 & 5.692 & 5.701 & 5.710 & 5.718 & 5.727 & $5.73^{6}$ & 2 & 2 \\
\hline 33. & 5.745 & 5.753 & $5 \cdot 7^{62}$ & $5 \cdot 77^{1}$ & 5.779 & 5.788 & 5.797 & 5.805 & 5.814 & 5.822 & 3 & 2 \\
\hline 34. & 5.831 & 5.840 & 5.848 & 5.857 & 5.865 & 5.874 & 5.882 & $5.89 \mathrm{I}$ & 5.899 & 5.908 & 4 & 3 \\
\hline 35. & 5.916 & 5.925 & 5.933 & $5.94 \mathrm{I}$ & 5.950 & $5.95^{8}$ & 5.967 & 5.975 & 5.983 & 5.992 & 5 & 4 \\
\hline 36. & 6. & 6 & 6. & 5 & 6. & & 0 & 8 & 6 & 5 & 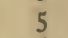 & 5 \\
\hline 37. & 6.083 & $6.09 x$ & 6.099 & 6.107 & $6.1: 6$ & 6. & 6.132 & 6.140 & 48 & 6.156 & 6 & 6 \\
\hline 38. & 6.164 & 6.173 & 6.181 & 6.189 & 6.197 & 6.2 & 6.213 & 6.221 & 6.229 & 6.237 & 7 & 6 \\
\hline 39. & 6.245 & 6.253 & 6.261 & 6.269 & 6.277 & 6.285 & 6.293 & 6.301 & 6.309 & 6.317 & 8 & 7 \\
\hline 40. & 6.325 & 6.332 & 6.340 & 6.348 & 6.356 & 4 & 6.372 & 6.380 & 6.387 & 6.395 & 8 & 7 \\
\hline 41. & 6.403 & 6.411 & 6.419 & 6.427 & 6.434 & $6.44^{2}$ & 6.450 & $6.45^{8}$ & $6.46_{5}$ & 6.473 & 1 & 1 \\
\hline 42. & $6.48 \mathrm{I}$ & 6.488 & 6.496 & 6.504 & 6.512 & 6.519 & 6.527 & 6.535 & 6.542 & $6.55^{\circ}$ & 2 & I \\
\hline 43. & 6.557 & 6.565 & 6.573 & 6.580 & 6.588 & 6.595 & 6.603 & $6.6 \mathrm{II}$ & 6.618 & 6.626 & 2 & 2 \\
\hline 44. & 6.633 & $6.64 I$ & 6.648 & 6.656 & 6.663 & $6.67 \mathrm{I}$ & 6.678 & 6.686 & 6.693 & 6.701 & 3 & 3 \\
\hline 45. & 6.708 & 6.716 & 6.723 & $6.73 \mathrm{I}$ & $6.73^{8}$ & 6.7 & 6.753 & 6.760 & 6.768 & 6.775 & 4 & 4 \\
\hline 46. & 6.782 & 6.790 & 6.797 & 6.804 & 6.812 & 6.819 & 6.826 & 6.834 & $6.84 \mathrm{I}$ & 6.848 & 5 & 4 \\
\hline 47. & 6.856 & 6.863 & 6.870 & 6.877 & 6.885 & 6.892 & 6.899 & 6.907 & 6.914 & 6.921 & 6 & 5 \\
\hline 48. & 6.928 & 6.935 & 6.943 & 6.950 & 6.957 & 6.964 & 6.971 & 6.979 & 6.986 & 6.993 & 6 & 6 \\
\hline 49. & 7.000 & 7.007 & 7.014 & 7.021 & 7.029 & 7.036 & 7.043 & 7.050 & 7.057 & 7.064 & 7 & 6 \\
\hline
\end{tabular}


SQ. RTS. \& SQRS.

\begin{tabular}{|c|c|c|c|c|c|c|c|c|c|c|c|c|}
\hline No. & 0 & I & 2 & 3 & 4 & 5 & 6 & 7 & 8 & 9 & \multicolumn{2}{|c|}{$\begin{array}{l}\text { Interpola, for } \\
\text { Hundredths. }\end{array}$} \\
\hline 50. & 7.071 & 7.078 & $7.08_{5}$ & 7.092 & 7.099 & 7.106 & 7.113 & 7.120 & 7.127 & 7.134 & 7 & 6 \\
\hline 51. & 7.141 & $7.1+8$ & 7.155 & 7.162 & 7.169 & 7.176 & $7.18_{3}$ & 7.190 & 7.197 & 7.204 & I & I \\
\hline 52. & 7.211 & 7.218 & 7.225 & 7.232 & 7.239 & 7.246 & 7.253 & 7.259 & 7.266 & 7.273 & I & $I$ \\
\hline 53. & 7.280 & $7.2 \mathrm{~S}_{7}$ & 7.294 & 7.301 & $7 \cdot 308$ & $7 \cdot 3^{14}$ & $7 \cdot 321$ & $7 \cdot 328$ & $7 \cdot 335$ & $7 \cdot 342$ & 2 & 2 \\
\hline 54. & $7 \cdot 348$ & $7 \cdot 355$ & $7 \cdot 362$ & $7 \cdot 369$ & $7 \cdot 37^{6}$ & $7 \cdot 3^{82}$ & $7 \cdot 3^{89} 9$ & $7 \cdot 396$ & 7.403 & 7.409 & 3 & 2 \\
\hline 55. & $7 \cdot 416$ & 7.423 & 7.430 & $7 \cdot 43^{6}$ & $7 \cdot 443$ & 7.450 & $7 \cdot 457$ & $7.46_{3}$ & $7 \cdot 470$ & $7 \cdot 477$ & 4 & 3 \\
\hline 56. & $7 \cdot 4^{8} 3$ & 7.490 & $7 \cdot 497$ & 7.503 & 7.510 & $7 \cdot 517$ & $7 \cdot 5^{23}$ & 7.530 & 7.537 & 7.543 & 4 & 4 \\
\hline 57. & 7.550 & $7.55^{6}$ & 7.563 & 7.570 & 7.576 & $7 \cdot 5^{8} 3$ & $7 \cdot 5^{89}$ & $7 \cdot 596$ & $7.60_{3}$ & 7.609 & 5 & 4 \\
\hline $5^{8 .}$ & 7.616 & 7.622 & 7.629 & 7.635 & 7.642 & 7.649 & 7.655 & 7.662 & 7.668 & 7.675 & 6 & 5 \\
\hline 59. & 7.681 & 7.689 & 7.694 & $7 \cdot 701$ & 7.707 & $7 \cdot 7^{14}$ & $7 \cdot 720$ & $7 \cdot 727$ & 7.733 & 7.740 & 6 & 5 \\
\hline 60. & $7 \cdot 746$ & $7.75^{2}$ & 7.759 & $7.76_{5}$ & 7.772 & $7 \cdot 77^{8}$ & 7.785 & $7 \cdot 791$ & 7.797 & 7.804 & 7 & 6 \\
\hline 61. & 7.810 & 7.817 & 7.823 & 7.829 & 7.836 & 7.842 & 7.849 & 7.855 & 7.861 & 7.868 & 1 & I \\
\hline 62. & $7: 874$ & 7.850 & 7.887 & 7.893 & 7.899 & 7.906 & 7.912 & 7.918 & 7.925 & 7.931 & 1 & 1 \\
\hline 63. & 7.937 & 7.944 & $7.95^{\circ}$ & 7.956 & 7.962 & 7.969 & 7.975 & 7.981 & 7.987 & 7.994 & 2 & 2 \\
\hline 64. & 8000 & 8.006 & 8.012 & 8.019 & 8.025 & 8.031 & 8.037 & 8.044 & 8.050 & 8.056 & 3 & 2 \\
\hline 65. & 8.062 & 8.068 & 8.075 & 8.081 & 8.087 & 8.093 & 8.099 & 8.106 & 8.112 & 8.118 & 4 & 3 \\
\hline 66. & 8.124 & 8.130 & 8.136 & 8.142 & 8.149 & 8.155 & 8.161 & 8.167 & 8.173 & 8.179 & 4 & 4 \\
\hline 67. & $8.15_{5}$ & 8.191 & 8.198 & 8.204 & 8.210 & 8.216 & 8.222 & 8.228 & 8.234 & 8.240 & 5 & 4 \\
\hline 68. & 8.246 & $8.25^{2}$ & $8.25^{8}$ & 8.264 & 8.270 & 8.276 & $8.28_{3}$ & 8.289 & 8.295 & 8.301 & 6 & 5 \\
\hline 69. & 8.307 & 8.313 & 8.319 & 8.325 & 8.331 & 8.337 & 8.343 & 8.349 & 8.355 & 8.361 & 6 & 5 \\
\hline 70. & 8. 367 & 8. 373 & 8.379 & $8.3^{8}{ }_{5}$ & 8.390 & 8.396 & 8.402 & 8.408 & 8.414 & 8.420 & 6 & 5 \\
\hline 71. & 8.426 & $8.43^{2}$ & $8.43^{8}$ & 8.444 & 8.450 & 8.456 & 8.462 & 8.468 & 8.473 & 8.479 & 1 & 1 \\
\hline 72. & 8.485 & 8.491 & 8.497 & 8.503 & 8.509 & 8.515 & $8.5^{21}$ & 8.526 & 8.532 & $8.53^{8}$ & 1 & I \\
\hline 73. & 8.544 & 8.550 & $8.55^{6}$ & 8.562 & 8.567 & 8.573 & 8.579 & 8. $5^{85}$ & 8.591 & 8.597 & 2 & 2 \\
\hline 74. & 8.602 & 8.603 & 8.614 & 8.620 & 8.626 & 8.631 & 8.637 & 8.643 & 8.649 & 8.654 & 2 & 2 \\
\hline 75. & 8.660 & 8.666 & 8.672 & $8.67^{8}$ & $8.68_{3}$ & 8.689 & 8.695 & 8.701 & 8.706 & 8.712 & 3 & 3 \\
\hline 76. & 8.718 & 8. $7^{2} 4$ & 8. 729 & 8.735 & 8.741 & 8.746 & $8.75^{2}$ & $8.75^{8}$ & 8.764 & 8.769 & 4 & 3 \\
\hline 77. & 8. 775 & 8. $7 \mathrm{Si}$ & 8.786 & 8. $79^{2}$ & 8. 798 & $8 . \mathrm{SO}_{3}$ & 8.809 & $8 . S_{15}$ & 8.820 & 8.826 & 4 & 4 \\
\hline 78. & $8.8_{32}$ & $8.8_{37}$ & 5.843 & $8 . \$ 49$ & $8 . S_{54}$ & 8.860 & 8.866 & $8 . S_{71}$ & 8.877 & $8.8 s_{3}$ & 5 & 4 \\
\hline 79. & 8.558 & 8.594 & 8.899 & S.yo5 & 8.911 & 8.916 & 8.922 & 8.927 & 8.933 & 8.939 & 5 & 5 \\
\hline 80. & 8.944 & 8.050 & 8.955 & 8.961 & 8.967 & 8.972 & 8. $) 7^{8}$ & $8.98_{3}$ & 8.989 & 8.994 & 6 & 5 \\
\hline Si. & 9.000 & 9.006 & 9.011 & 9017 & 9.022 & 9.028 & 9.033 & 9.039 & 9.044 & 9.050 & I & 1 \\
\hline 82. & 9055 & 9.061 & 9.066 & $9.07^{2}$ & 9.077 & $9.0 \$_{3}$ & 9.088 & 9.094 & 9.099 & 9.105 & 1 & I \\
\hline 83. & 9.110 & 9.116 & 9.121 & 9.127 & 9.132 & $9.13^{8}$ & 9.143 & 9.149 & 9.154 & 9.160 & 2 & 2 \\
\hline 84. & 9.165 & 9.171 & 9.176 & 9.182 & 9.187 & 9.192 & 9.198 & 9.203 & 9.209 & 9.214 & 2 & 2 \\
\hline $8_{5}$. & 9.220 & 9.225 & 9.230 & 9.236 & 9.241 & 9.247 & 9.252 & 9.257 & 9.26 .3 & 9.268 & 3 & 3 \\
\hline 86. & 9.274 & $9.27^{\circ}$ & 9.284 & 9.290 & 9.295 & 9.301 & 9.306 & 9.311 & 9.317 & $9 \cdot 322$ & 4 & 3 \\
\hline 87. & 9.327 & $9 \cdot 333$ & $9.33^{\mathrm{s}}$ & $9 \cdot 343$ & $9 \cdot 349$ & $9 \cdot 354$ & $9 \cdot 359$ & 9.365 & 9.370 & $9 \cdot 375$ & 4 & 4 \\
\hline 88. & $9 \cdot 3^{88}$ & $9 \cdot 386$ & 9. 391 & 9.397 & 1) 402 & 9.407 & 9.413 & $9 \cdot 418$ & 9.423 & $9.421)$ & 5 & 4 \\
\hline 89. & $9 \cdot 4.34$ & 9.439 & 9.445 & 9.450 & 9.455 & 9.460 & 9.466 & 9.471 & $9 \cdot 476$ & 9.482 & 5 & 5 \\
\hline 90. & 9.487 & 9.492 & 9.497 & 9.503 & 9.508 & 9.513 & 9.518 & 9.524 & 9.529 & 9.5 .34 & 5 & \\
\hline 91. & 9.539 & 9.545 & 9.550 & 9.555 & 9.560 & 9.566 & 9.571 & 9.576 & $9.5^{81}$ & 9.586 & 1 & \\
\hline 92. & 9.592 & 9.597 & 9.602 & 9.607 & 9.612 & 9.618 & 9.623 & $9.6 .2 \mathrm{~S}$ & 9.63 .3 & 9.638 & 1 & \\
\hline 93. & 9.644 & 9.649 & 9.654 & 9.659 & 9.664 & 9.670 & 9.675 & 9.6800 & $9.6 \$_{5}$ & $9.6 \mathrm{~kg}$ & 2 & \\
\hline 94. & 9.695 & 9.701 & 9706 & 9.711 & 9.716 & 9.721 & 9.726 & 9.731 & 9.737 & 9.742 & 2 & \\
\hline 95. & 9.747 & 9.752 & 9.757 & $9 \cdot 7^{6 / 2}$ & 9.767 & 9.772 & $9.77^{8}$ & 9.783 & 9.788 & 9.793 & 3 & \\
\hline 96. & 9.798 & $9 \mathrm{SO}_{3}$ & 9508 & $9 . \$ 13$ & 9.815 & 9.823 & 9.829 & $9.8_{34}$ & 9.839 & 9.844 & 3 & \\
\hline 97. & 9.8 .49 & 9.854 & $9 . \$ 50$ & 9.864 & 9.869 & 9.874 & 9.879 & $9.8 s_{4}$ & 9.8Sy & 9.S94 & 4 & \\
\hline gs. & 9.899 & 9.005 & 9.010 & 9.915 & 0.920 & $9.9) 25$ & y.9.30 & 9.035 & 9.940 & 9.945 & 4 & 1 \\
\hline 99. & $9.95^{\circ}$ & 9.955 & 9.960 & 9.965 & 9.970 & 9.975 & 9980 & $9.98_{5}$ & 9.0000 & 9.995 & 5 & 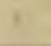 \\
\hline
\end{tabular}




\section{RECIPROCALS.}

RECIP.

\begin{tabular}{|c|c|c|c|c|c|c|c|c|c|c|c|c|c|c|}
\hline No. & 0 & I & 2 & 3 & 4 & 5 & 6 & 7 & 8 & 9 & \multicolumn{4}{|c|}{$\begin{array}{l}\text { INTERPOLATION } \\
\text { TABLES. }\end{array}$} \\
\hline 00 & \multicolumn{5}{|c|}{0.99900 .99800 .99700 .9960} & \multicolumn{5}{|c|}{0.99500 .99400 .99300 .99210 .991 I } & \multicolumn{4}{|c|}{$-85-75-65-55$} \\
\hline ol & 0.9901 & $9 S_{91}$ & $9 S S I$ & $9 \$ 72$ & 9502 & $9 S_{52}$ & 9843 & 9833 & 9823 & 9814 & 9 & 8 & 7 & \\
\hline .02 & $9 \mathrm{SO}_{4}$ & 9794 & & 9775 & 9766 & 9756 & 9747 & & & & 17 & 15 & 13 & II \\
\hline .03 & 9709 & 9699 & & & & & & & 9634 & 9625 & 26 & 23 & 20 & \\
\hline .04 & 9615 & 9606 & 9597 & $95^{88}$ & 9579 & 9569 & 9560 & $955^{1}$ & $954^{2}$ & 9533 & 34 & 30 & 26 & 22 \\
\hline 1.05 & 0.9524 & .9515 & .95060 & 0.94970 & .9488 & 0.94790 & .94700 & $0.946 \mathrm{I} \mathrm{C}$ & .94520 & .9443 & 43 & $3^{8}$ & 33 & \\
\hline .06 & 9434 & 9425 & 9416 & 9407 & 9399 & & & & & & $5^{1}$ & 45 & 39 & 33 \\
\hline .07 & 9346 & 933 & & & & & & & & & 60 & 53 & 46 & 39 \\
\hline .08 & 9259 & 9251 & 9242 & 9234 & 9225 & & 9000 & & 9191 & 183 & 68 & 60 & $5^{2}$ & 44 \\
\hline .09 & 9174 & 9166 & $915^{8}$ & 9149 & $914 \mathrm{I}$ & 2 & 9124 & 91 & 9107 & 099 & 77 & 68 & 59 & 50 \\
\hline 0 & \multicolumn{5}{|c|}{0.99010 .98040 .97090 .9615} & \multicolumn{5}{|c|}{0.95240 .94340 .93460 .92590 .9174} & \multicolumn{4}{|c|}{$-45-35-25-22$} \\
\hline .1 & 0.9091 & 9009 & 8929 & 8850 & $877^{2}$ & 8696 & I & 8547 & 8475 & 8403 & 5 & 4 & 3 & 2 \\
\hline .2 & 8333 & 8264 & 8197 & 81 & $806_{5}$ & 8000 & 7937 & & & & 9 & 7 & 5 & 4 \\
\hline$\cdot 3$ & 7692 & 7634 & 75 & 75 & & & & & & & I4 & II & 8 & 7 \\
\hline .4 & 7143 & 7092 & $704^{2}$ & 6993 & 6944 & & & & 6757 & 6711 & 18 & 14 & 10 & 9 \\
\hline 1.5 & \multicolumn{5}{|c|}{0.66670 .66230 .65790 .65360 .6494} & \multicolumn{5}{|c|}{$0.645^{2} 0.64100 .63690 .63290 .6289$} & 23 & 18 & 13 & \\
\hline .6 & 6250 & 6211 & 6173 & 6135 & & & & & 5952 & 5917 & 27 & 21 & 15 & 13 \\
\hline .7 & $5^{882}$ & 5848 & $5^{814}$ & & 5 & & & & & & 32 & 25 & 18 & 15 \\
\hline .8 & 5 & 55 & & & & & & & & & 36 & 28 & 20 & 18 \\
\hline 0 & 3 & $5^{2}$ & & & $5^{1}$ & & & & & & $4 \mathrm{I}$ & 32 & 23 & 0 \\
\hline 2.0 & \multicolumn{5}{|c|}{0.50000 .49750 .49500 .49260 .4902} & \multicolumn{5}{|c|}{0.48780 .48540 .48310 .48080 .4785} & \multicolumn{4}{|c|}{$-18-16-14-12$} \\
\hline .1 & 4762 & 47 & 4717 & 4695 & 4673 & 46 & 4630 & 4608 & $45^{87}$ & 4566 & & 2 & & I \\
\hline .2 & 4545 & 45 & 4 & 44 & 4 & 4 & 4 & & & & 4 & 3 & 3 & 2 \\
\hline$\cdot 3$ & 4. & 43 & 43 & & & & & & & & 5 & 5 & 4 & 4 \\
\hline .4 & 4167 & 4149 & 4132 & 4115 & 4098 & (1) & 4005 & 4049 & 4032 & 4016 & 7 & 6 & 6 & 5 \\
\hline 2.5 & \multicolumn{5}{|c|}{0.40000 .39840 .39680 .39530 .3937} & \multicolumn{5}{|c|}{0.39220 .39060 .38910 .38760 .3861} & 9 & 8 & 7 & 6 \\
\hline .6 & 3846 & $383 I$ & 3817 & 3802 & & 3774 & 3759 & 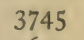 & 3731 & 3717 & II & 10 & 8 & 7 \\
\hline .7 & 3 & 36 & & & & & & & & & I3 & II & 10 & 8 \\
\hline .8 & & 35 & 35 & & & & & & & & 14 & 13 & I I & 10 \\
\hline$c_{2}>$ & 3 & 34 & & & & & & & & & 16 & 14 & 13 & II \\
\hline 3.0 & \multicolumn{5}{|c|}{0.33330 .33220 .331110 .33000 .3289} & \multicolumn{5}{|c|}{0.32790 .32680 .32570 .32470 .3236} & -11 & -9 & -8 & -7 \\
\hline .1 & 3226 & 3215 & 3205 & & 3185 & & & & & & I & $\mathbf{I}$ & I & 1 \\
\hline .2 & 312 & $3 i$ & & & & & & & & & 2 & 2 & 2 & I \\
\hline$\cdot 3$ & 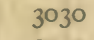 & 302 & 30 & & & & & & & & 3 & 3 & 2 & 2 \\
\hline .4 & 2941 & 2933 & 2924 & 2 & & & & & & & 4 & 4 & 3 & 3 \\
\hline 3.5 & 0.2857 & 0.2849 & .28410 & 0.28330 & .2825 & 0.28 & .28090 & .28 & .27930 & .2786 & 6 & 5 & 4 & 4 \\
\hline 6 & $277^{8}$ & 2770 & 2762 & & 2747 & & & & & & 7 & 5 & 5 & 4 \\
\hline 1 & & 26 & & & & & & & & & 8 & 6 & 6 & \\
\hline .8 & 2632 & 2625 & 261 & 26 & 26 & & & 2 & & & 9 & 7 & 6 & 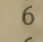 \\
\hline .9 & 2564 & $255^{8}$ & 255 & 25 & $253^{8}$ & 2 & 2 & 25 & 2513 & 2506 & 10 & 8 & 7 & 6 \\
\hline 4.0 & 0.2500 & .2494 & .24000 & 0.24 & 0.24 & .24 & 0.22 & & .24 & & -6 & -5 & $x$ & \\
\hline .I & 2439 & 2433 & 2427 & & 24 & 2410 & 24 & 2398 & 2392 & 2387 & I & I & 0 & \\
\hline .2 & $238 \mathrm{I}$ & 2375 & 23 & & 2 & & & & 2336 & 2331 & I & I & I & \\
\hline$\cdot 3$ & 2326 & 232 & & 23 & & & & 38 & 2283 & 2278 & 2 & 2 & 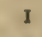 & \\
\hline .4 & 2273 & 2268 & 2262 & 2257 & 2252 & 2247 & 2242 & 2237 & 2232 & 2227 & 2 & 2 & 2 & \\
\hline $4 \cdot 5$ & 0.2222 & .2217 & .2212 & 0.22080 & .2203 & .21980 & .21930 & .21880 & .21830 & .2179 & 3 & 3 & 2 & \\
\hline .6 & 2174 & 2169 & 2165 & 2160 & 2155 & 2151 & 2146 & $214 \mathrm{I}$ & 2137 & 2132 & 4 & 3 & 2 & \\
\hline 17 & 2128 & 212 & $2 \mathrm{I}$ & & & & & 2096 & & 2088 & 4 & 4 & 3 & \\
\hline .8 & 2083 & 2079 & 207 & & 20 & 20 & 2058 & 2053 & & 2045 & 5 & 4 & 3 & \\
\hline .9 & 2041 & 2037 & 2033 & 2028 & 2024 & 2020 & 2016 & 2012 & 2008 & 2004 & 5 & 5 & r & \\
\hline
\end{tabular}




\begin{tabular}{|c|c|c|c|c|c|c|c|c|c|c|c|c|}
\hline No. & 0 & $\mathbf{I}$ & 2 & 3 & 4 & 5 & 6 & 7 & 8 & 9 & \multicolumn{2}{|c|}{$\begin{array}{l}\text { INTERPOLATION N } \\
\text { FOR THOUS. }\end{array}$} \\
\hline 5.0 & \multicolumn{5}{|c|}{0.20000 .19960 .19920 .19880 .1984} & \multicolumn{5}{|c|}{0.19800 .19760 .19720 .19690 .1965} & -4 & -3 \\
\hline 1 & 1961 & 1957 & 1953 & 1949 & 1946 & 1942 & 1938 & 1934 & 1931 & 1927 & o & 0 \\
\hline .2 & 1923 & 1919 & 1916 & 1912 & 1908 & 1905 & 1901 & 1898 & 1894 & 1890 & 1 & I \\
\hline .3 & 1887 & $18 S_{3}$ & 1580 & 1876 & 1873 & 1869 & 1866 & 1862 & 1859 & 1855 & I & $I$ \\
\hline .4 & 1852 & 1848 & 1845 & 1842 & 1838 & 1835 & 1832 & 1828 & 1825 & 1821 & 2 & I \\
\hline $5 \cdot 5$ & \multicolumn{5}{|c|}{0.18180 .18150 .18120 .18050 .1805} & \multicolumn{5}{|c|}{0.18020 .17990 .17950 .17920 .1789} & 2 & 2 \\
\hline .6 & 1786 & $178_{3}$ & 1779 & 1776 & 1773 & $177^{\circ}$ & 1767 & 1764 & 1761 & 1757 & 2 & 2 \\
\hline .7 & 1754 & 1751 & 1748 & 1745 & 1742 & 1739 & 1736 & 1733 & 1730 & 1727 & 3 & 2 \\
\hline .8 & 1724 & 1721 & 1718 & 1715 & 1712 & 1709 & 1706 & 1704 & 1701 & 1698 & 3 & 2 \\
\hline .9 & 1695 & $16 g_{2}$ & 1689 & 1656 & 1684 & $168 I$ & 1678 & 1675 & 1672 & 1669 & 4 & 3 \\
\hline 6.0 & \multicolumn{5}{|c|}{0.16670 .16640 .16610 .16580 .1656} & \multicolumn{5}{|c|}{0.16530 .16500 .16470 .16450 .1642} & -3 & -2 \\
\hline .1 & 1639 & 1637 & 1634 & 1631 & 1629 & 1626 & 1623 & 1621 & 1618 & 1616 & o & o \\
\hline .2 & 1613 & 1610 & 1608 & $160_{5}$ & 1603 & 1600 & 1597 & 1595 & 1592 & 1590 & I & o \\
\hline$\cdot 3$ & 1587 & $15{ }^{8} 5$ & $15^{82}$ & $15 S 0$ & 1577 & 1575 & 1572 & 1570 & 1567 & 1565 & I & 1 \\
\hline .4 & 1563 & 1560 & $155^{8}$ & $\mathbf{I} 555$ & 1553 & $155^{\circ}$ & 1548 & 1546 & I 543 & 1541 & I & I \\
\hline 6.5 & \multicolumn{5}{|c|}{0.15380 .15360 .15340 .15310 .1529} & \multicolumn{5}{|c|}{0.15270 .15240 .15220 .15200 .1517} & 2 & 1 \\
\hline .6 & 1515 & 1513 & 1511 & 1508 & 1506 & 1504 & 1502 & 1499 & 1497 & 1495 & 2 & $\mathbf{I}$ \\
\hline .7 & 1493 & 1490 & 1488 & 1486 & 1484 & 1481 & 1479 & 1477 & 1475 & 1473 & 2 & 1 \\
\hline .8 & 1471 & 1468 & 1466 & 1464 & 1462 & 1460 & $145^{8}$ & $145^{6}$ & 1453 & $145^{1}$ & 2 & 2 \\
\hline .9 & 1449 & 1447 & 1445 & 1443 & $144^{I}$ & 1439 & 1437 & 1435 & 1433 & 1431 & 3 & 2 \\
\hline 7.0 & \multicolumn{5}{|c|}{0.14290 .14270 .14250 .14220 .1420} & \multicolumn{5}{|c|}{0.14180 .14160 .14140 .14120 .1410} & -2 & -1 \\
\hline .1 & 1408 & 1406 & 1404 & 1403 & 1401 & I 399 & 1397 & I 395 & 1393 & I 391 & 0 & 0 \\
\hline .2 & I 389 & $13^{87}$ & $1_{3} 8_{5}$ & 1383 & $138 \mathbf{I}$ & 1379 & 1377 & 1376 & 1374 & 1372 & o & 0 \\
\hline$\cdot 3$ & 1370 & 1368 & I 366 & 1364 & 1362 & 1361 & 1359 & I 357 & 1355 & I353 & 1 & 0 \\
\hline .4 & 1351 & 1350 & 1348 & 1346 & 1344 & I 342 & I 340 & 1339 & I 337 & I 335 & $\mathbf{I}$ & 0 \\
\hline $7 \cdot 5$ & \multicolumn{5}{|c|}{0.13330 .13320 .13300 .13280 .1326} & \multicolumn{5}{|c|}{0.13250 .13230 .13210 .13190 .1318} & $\mathbf{I}$ & I \\
\hline .6 & 1316 & 1314 & 1312 & 1311 & 1309 & 1307 & I 305 & 1304 & 1302 & 1300 & I & $\mathbf{I}$ \\
\hline$\cdot 7$ & 1299 & 1297 & 1295 & 1294 & 1292 & 1290 & 1289 & 1287 & 1285 & 1284 & I & I \\
\hline .8 & $12 S_{2}$ & 1280 & 1279 & 1277 & $127^{6}$ & 1274 & 1272 & 1271 & 1269 & 1267 & 2 & $\mathbf{I}$ \\
\hline .9 & 1266 & 1264 & 1263 & 1261 & 1259 & 1258 & 1256 & 1255 & 1253 & 1252 & 2 & I \\
\hline 8.0 & \multicolumn{5}{|c|}{0.12500 .12480 .12470 .12450 .1244} & \multicolumn{5}{|c|}{0.12420 .12410 .12390 .12380 .1236} & -8 & -1 \\
\hline.$I$ & 1235 & 1233 & 1232 & 1230 & 1229 & 1227 & 1225 & 1224 & 1222 & 1221 & 0 & 0 \\
\hline .2 & 1220 & 1218 & 1217 & 1215 & 1214 & 1212 & 1211 & 1209 & 1208 & 1206 & o & o \\
\hline$\cdot 3$ & 1205 & $12 O_{3}$ & 1202 & 1200 & 1109 & 1198 & 1196 & 1195 & 1193 & 1192 & 1 & 0 \\
\hline .4 & 1100 & 1189 & 1188 & I 186 & 1185 & 1183 & 1182 & 1181 & 1179 & $117^{8}$ & 1 & 0 \\
\hline 8. 5 & \multicolumn{5}{|c|}{0.11760 .11750 .11740 .11720 .1171} & \multicolumn{5}{|c|}{0.11700 .11680 .11670 .11660 .1164} & I & 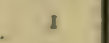 \\
\hline .6 & 1163 & 1161 & 1160 & 1159 & 1157 & 1156 & II 55 & 1153 & 1152 & 1151 & 1 & I \\
\hline .7 & 11.49 & 1148 & 1147 & 1145 & 1144 & 1143 & 1142 & 1140 & 1139 & 1138 & 1 & I \\
\hline .8 & 11,36 & 11,35 & 1134 & 1133 & 1131 & 1130 & 1129 & 1127 & 1126 & 1125 & 2 & 1 \\
\hline .9 & 1124 & 1822 & 1121 & 8120 & 1119 & 8187 & 1816 & 8185 & 1114 & 1112 & 2 & 1 \\
\hline 9.0 & о ий & .11100 & 0.11090 & .11070 & 0.1106 & 0.11050 & $.180_{4} 0$ & .11030 & . I IOI 0. & .1100 & -2 & -1 \\
\hline .1 & $10 \times 19$ & Ior): & $\operatorname{sog} \theta$ & 1095 & 1094 & 1093 & $\log 2$ & 1001 & 1080 & ross & 0 & 0 \\
\hline .2 & 1087 & $\ln 86$ & $\operatorname{los}_{5}$ & $\operatorname{los}_{3}$ & $10 S_{2}$ & 1081 & 1050 & 1079 & 1078 & 1076 & 0 & 0 \\
\hline$\cdot 3$ & 1075 & 1074 & 1073 & 1072 & 1071 & 1070 & 1068 & 1067 & 1066 & 1065 & 1 & 0 \\
\hline 4 & 1064 & 1063 & 1062 & 10\% & 1059 & $\operatorname{cog} 8$ & 1057 & 1056 & 1055 & 1054 & 1 & 0 \\
\hline $9 \cdot 5$ & 0.10530 & .10520 & $0.1050 \mathrm{C}$ & 10490 & 0.1048 & 0.10470 & .10460 & 10.450 & .10 .440 & .1043 & 1 & I \\
\hline .6 & 1042 & 1041 & 10.90 & 1038 & 1037 & $103^{6}$ & 1035 & 10.34 & 1033 & 1032 & 1 & 1 \\
\hline$\cdot 7$ & 1031 & 10,30 & 1029 & 1028 & 1027 & 1026 & 1025 & 1024 & 1022 & 1021 & 1 & 1 \\
\hline .8 & 1020 & 1019 & 1018 & 1017 & 1016 & rot5 & 1014 & 1013 & 1012 & 1011 & 2 & I \\
\hline .9 & 1010 & 1009 & 1008 & 1007 & 1006 & 1005 & 1004 & 1003 & 1002 & 1001 & 2 & 1 \\
\hline
\end{tabular}


SLIDE-WIRE RATIOS.

S. W. RATIOS.

\begin{tabular}{|c|c|c|c|c|c|c|c|c|c|c|}
\hline $\mathrm{cm}$. & $\mathrm{o}^{\mathrm{mm}}$. & I $\mathrm{mm}$. & $2^{\mathrm{mm}}$. & $3^{\mathrm{mm}}$. & $4^{\mathrm{mm}}$. & $5^{\mathrm{mm} .}$ & $6^{\mathrm{mm}}$. & $7^{\mathrm{mm}}$ & $8^{\mathrm{mm}}$. & $9^{\mathrm{mm}}$. \\
\hline 0 & 0.0000 & 0.0010 & 0.0020 & 0.0030 & 0.0040 & 0.0050 & 0.0060 & 0.0071 & 0.0081 & 0.0091 \\
\hline I & oror & OIII & 0122 & or 32 & OI 42 & 0152 & or63 & o173 & $018_{3}$ & OI94 \\
\hline 2 & 0204 & 0215 & 0225 & 0235 & 0246 & 0256 & 0267 & 0278 & 0288 & 0299 \\
\hline 3 & 0309 & 0320 & O331 & 0341 & 0352 & 0363 & 0373 & $\mathrm{O}_{3} 84$ & 0395 & 0406 \\
\hline 4 & 0417 & 0428 & $043^{8}$ & 0449 & 0460 & $047^{1}$ & 0482 & 0493 & 0504 & 0515 \\
\hline 5 & 0.0526 & 0.0537 & 0.0549 & 0.0560 & 0.0571 & $0.05^{82}$ & 0.0593 & 0.0605 & 0.0616 & 0.0627 \\
\hline 6 & $063^{8}$ & 0650 & o66r & 0672 & 0684 & 0695 & 0707 & 0718 & 0730 & 0741 \\
\hline 7 & 0753 & 0764 & 0776 & 0788 & 0799 & 0811 & 0823 & 0834 & 0846 & 0858 \\
\hline 8 & $0 \$_{70}$ & $088 \mathrm{I}$ & 0893 & 0905 & 0917 & 0929 & 0941 & 0953 & 0965 & 0977 \\
\hline 9 & 0989 & IOOI & 1013 & 1025 & $103^{8}$ & 1050 & 1062 & 1074 & 1087 & 1099 \\
\hline 10 & O.IIII & 0. 1124 & 0.1136 & 0.1148 & $0.116 x$ & 0.1173 & 0.1186 & 0.I198 & 0.1211 & 0.1223 \\
\hline II & 1236 & 1249 & I261 & 1274 & 1287 & 1299 & 1312 & 1325 & - 1338 & $135^{I}$ \\
\hline 12 & 1364 & 1377 & 1390 & 1403 & 1416 & 1429 & 1442 & I 455 & 1468 & $148 \mathrm{I}$ \\
\hline 13 & I 494 & 1508 & 1521 & 1534 & 1547 & 1561 & 1574 & 1588 & 1601 & 1614 \\
\hline 14 & $\mathbf{I} 628$ & 1641 & 1655 & 1669 & 1682 & I 696 & 1710 & 1723 & 1737 & 1751 \\
\hline 15 & 0.1765 & 0.1779 & 0.1793 & 0.1806 & 0.1820 & 0.1834 & 0.1848 & 0.1862 & 0.1877 & 0.1891 \\
\hline 16 & 1905 & 1919 & 1933 & 1947 & 1962 & 1976 & 1990 & 2005 & 2019 & 2034 \\
\hline 17 & 2048 & 2063 & 2077 & 2092 & 2107 & 2121 & 2136 & 2151 & 2166 & 2180 \\
\hline 18 & 2195 & 2210 & 2225 & 2240 & 2255 & $2270^{\circ}$ & 2285 & 2300 & 2315 & 2331 \\
\hline 19 & 2346 & 2361 & 2376 & 2392 & 2407 & 2422 & 2438 & 2453 & 2469 & 2484 \\
\hline 20 & 0.2500 & 0.2516 & 0.2531 & 0.2547 & 0.2563 & 0.2579 & 0.2595 & 0.2610 & 0.2626 & 0.2642 \\
\hline 21 & $265^{8}$ & 2674 & 2690 & 2707 & 2723 & 2739 & 2755 & 2771 & 2788 & 2804 \\
\hline 22 & $282 I$ & 2837 & 2854 & 2870 & 2887 & 2903 & 2920 & 2937 & 2953 & 2970 \\
\hline 23 & 2987 & 3004 & $302 \mathrm{I}$ & 3038 & 3055 & 3072 & 3089 & 3106 & 3123 & $314 I$ \\
\hline 24 & 3158 & 3175 & $3^{193}$ & 3210 & 3228 & 3245 & 3263 & 3280 & 3298 & 3316 \\
\hline 25 & 0.3333 & $0.335^{1}$ & 0.3369 & 0.3387 & 0.3405 & 0.3423 & 0.344 I & 0.3459 & 0.3477 & 0.3495 \\
\hline 26 & $35^{14}$ & 3532 & $355^{\circ}$ & 3569 & $35^{87}$ & 3605 & - 3624 & 3643 & 3661 & 3680 \\
\hline 27 & 3699 & 3717 & 3736 & 3755 & 3774 & 3793 & $3^{812}$ & $3^{8} 3^{1}$ & $3^{850}$ & 3870 \\
\hline 28 & 3889 & 3908 & 3928 & 3947 & 3967 & 3986 & 4006 & 4025 & 4045 & 4065 \\
\hline 29 & 4085 & 4104 & 4124 & $4 \mathrm{I} 44$ & 4164 & 4184 & 4205 & 4225 & 4245 & 4265 \\
\hline 30 & 0.4286 & 0.4306 & 0.4327 & 0.4347 & 0.4368 & $0.43^{89}$ & 0.4409 & 0.4430 & $0.445^{I}$ & 0.4472 \\
\hline $3 \mathrm{I}$ & 4493 & $45^{14}$ & 4535 & 4556 & 4577 & 4599 & 4620 & $464 I$ & 4663 & 4684 \\
\hline 32 & 4706 & 4728 & 4749 & $477^{1}$ & 4793 & $48 I_{5}$ & 4837 & 4859 & $488 I$ & 4903 \\
\hline 33 & 4925 & 4948 & 4970 & 4993 & 5015 & 5038 & 5060 & 5083 & $5^{106}$ & $5^{129}$ \\
\hline 34 & 5152 & $5^{175}$ & $5^{198}$ & 5221 & 5244 & 5267 & 5291 & 5314 & 5337 & 5361 \\
\hline 35 & 0.5385 & 0.5408 & $0.543^{2}$ & 0.5456 & 0.5480 & 0.5504 & $0.55^{28}$ & $0.555^{2}$ & 0.5576 & 0.5601 \\
\hline 36 & 5625 & 5650 & $5^{674}$ & 5699 & 5723 & 5748 & 5773 & 5798 & $5^{823}$ & 5848 \\
\hline 37 & $5^{873}$ & $5^{898}$ & 5924 & 5949 & 5974 & 6000 & 6026 & 6051 & 6077 & 6103 \\
\hline 38 & 6129 & 6155 & 6181 & 6208 & 6234 & 6260 & 6287 & 6313 & 6340 & 6367 \\
\hline 39 & 6393 & 6420 & 6447 & 6475 & 6502 & 6529 & $655^{6}$ & 6584 & 6611 & 6639 \\
\hline 40 & 0.6667 & 0.6695 & 0.6722 & $0.675^{\circ}$ & 0.6779 & 0.6807 & 0.6835 & 0.6863 & 0.6892 & 0.6921 \\
\hline $4 I$ & 6949 & 6978 & 7007 & 7036 & 7065 & 7094 & 7123 & 7153 & 7182 & 7212 \\
\hline 42 & $724 I$ & 7271 & 7301 & 7331 & 7361 & 7391 & 7422 & $745^{2}$ & $748_{3}$ & $75^{13}$ \\
\hline 43 & 7544 & 7575 & 7606 & 7637 & 7668 & 7699 & $773 \mathrm{I}$ & 7762 & 7794 & 7825 \\
\hline 44 & 7857 & 7889 & 7921 & 7953 & 7986 & 8018 & $80_{5} \mathrm{I}$ & $808_{3}$ & 8116 & 8149 \\
\hline 45 & 0.8182 & 0.8215 & 0.8248 & 0.8282 & 0.8315 & 0.8349 & 0.8382 & 0.8416 & $0.845^{\circ}$ & 0.8484 \\
\hline 46 & 8519 & 8553 & 8587 & 8622 & 8657 & 8692 & 8727 & 8762 & 8797 & 8832 \\
\hline 47 & 8868 & 8904 & 8939 & 8975 & 9011 & 9048 & 9084 & $912 I$ & 9157 & 9194 \\
\hline 48 & 9231 & 9268 & 9305 & 9342 & 9380 & 9418 & 9455 & 9493 & $953^{1}$ & 9570 \\
\hline 49 & 9608 & 9646 & 9685 & 9724 & 9763 & 9802 & $984 \mathrm{I}$ & $988 \mathbf{I}$ & 9920 & 9960 \\
\hline
\end{tabular}




\section{SLIDE-WIRE RATIOS.}

S. W. RATIOS.

\begin{tabular}{|c|c|c|c|c|c|c|c|c|c|c|}
\hline $\mathrm{cm}$. & $0^{\mathrm{mm}}$. & I mm. & $2^{\mathrm{mm}}$ & $3^{\mathrm{mm}}$ & $4^{\mathrm{mm}}$. & $5^{\mathrm{mm}}$ & $6^{\mathrm{mi}}$ & $7^{\mathrm{mm}}$ & $8^{\mathrm{mm}}$. & \\
\hline 50 & 1.000 & 1.004 & 1.008 & 1.012 & 1.016 & 1.020 & 1.024 & 1.028 & 1.033 & 1.037 \\
\hline $5^{1}$ & 1.041 & 1.045 & 1.049 & 1.053 & $1.05^{8}$ & 1.062 & 1.066 & 1.070 & 1.075 & 1.079 \\
\hline $5^{2}$ & 1.083 & 1.088 & 1.092 & 1.096 & I.IOI & 1.105 & 1.110 & 1.114 & 1.119 & 1.123 \\
\hline 53 & $1.12 \mathrm{~S}$ & 1.132 & 1.137 & 1.141 & 1.146 & $1.15^{1}$ & 1.155 & 1.160 & $1.16_{5}$ & 1.169 \\
\hline 54 & 1.174 & 1.179 & 1.183 & 1.188 & 1.193 & 1.198 & 1.203 & 1.208 & 1.212 & 1.217 \\
\hline 55 & 1.222 & 1.227 & 1.232 & 1.237 & 1.242 & 1. 247 & 1.252 & 1.257 & 1.262 & 1.268 \\
\hline $5^{6}$ & 1.273 & 1.278 & 1.283 & 1.288 & 1.294 & 1.299 & 1.304 & 1.309 & 1.315 & 1.320 \\
\hline 57 & 1.326 & 1.331 & 1.336 & I. 342 & 1.347 & I. 353 & 1. $35^{8}$ & 1.364 & $1.37^{\circ}$ & 1.375 \\
\hline $5^{8}$ & $1 \cdot 3^{8 I}$ & 1.387 & I. 392 & I. $39^{8}$ & 1.404 & 1.410 & 1.415 & 1.421 & 1.427 & 1.433 \\
\hline 59 & 1.439 & 1.445 & I. $45^{1}$ & 1.457 & 1.463 & 1.469 & 1.475 & I. $48 \mathrm{I}$ & 1.488 & 1.494 \\
\hline 60 & 1.500 & 1. 506 & 1.513 & 1.519 & 1.525 & 1. $53^{2}$ & $1.53^{8}$ & I. 545 & $1.55^{1}$ & $1.55^{8}$ \\
\hline 61 & 1.564. & 1.571 & I. 577 & 1.584 & 1.591 & 1. & & II & & 1.625 \\
\hline 62 & 1.632 & 1.639 & 1.646 & 1. 653 & 1.660 & 1.667 & 1.674 & $1.68 \mathrm{I}$ & & 1.695 \\
\hline 63 & 1.703 & 1.710 & 1.717 & 1.725 & 1.732 & 1.740 & 1.747 & I. 755 & 1.762 & 1.770 \\
\hline 64 & $1.77^{8}$ & 1.786 & 1.793 & $1.80 \mathrm{I}$ & 1.809 & 1.817 & 1.825 & 1.833 & 1.841 & 1.849 \\
\hline 65 & 1.857 & 1.865 & 1.874 & 1.882 & 1.890 & 1.899 & 1.907 & 1.915 & 1.924 & 1.933 \\
\hline 66 & 1.941 & 1.950 & 1.959 & 1.967 & 1.976 & 1.985 & I.994 & 2.003 & 2.012 & 2.021 \\
\hline 67 & 2.030 & 2.040 & 2.049 & $2.05^{8}$ & 2.067 & 2.077 & 2.086 & 2.096 & 2. 106 & 2.115 \\
\hline 68 & 2.125 & 2.135 & 2.145 & 2.155 & 2.165 & 2.175 & 2.185 & 2.195 & 2.205 & 2.215 \\
\hline 69 & 2.226 & 2.236 & 2.247 & 2.257 & 2.268 & 2.279 & 2.289 & 2.300 & 2.311 & 2.322 \\
\hline 70 & 2.333 & 2.344 & 2.356 & 2.367 & 2.378 & 2. 390 & 2.401 & 2.413 & 2.425 & 2.436 \\
\hline 71 & $2.44^{8}$ & 2.460 & 2.472 & 2.484 & 2.497 & 2.509 & 2.521 & 2.534 & 2.546 & 2.559 \\
\hline 72 & 2.571 & $2.5^{84}$ & 2.597 & 2.610 & 2.623 & 2.636 & 2.650 & $2.66_{3}$ & 2.676 & 2.690 \\
\hline 73 & 2.704 & 2.717 & 2.731 & 2.745 & 2.759 & 2.774 & 2.788 & 2.802 & 2.817 & 2.831 \\
\hline 74 & 2.846 & $2.86 \mathrm{I}$ & 2.876 & $2.89 \mathrm{I}$ & 2.906 & 2.922 & 2.937 & 2.953 & 2.968 & 2.984 \\
\hline 75 & 3.000 & 3.016 & 3.032 & 3.049 & 3.065 & 3.082 & 3.098 & 3.115 & 2 & 3.149 \\
\hline 76 & 3.167 & 3.184 & 3.202 & 3.219 & 3.237 & 3.255 & 3.274 & 3.292 & & 3.329 \\
\hline 77 & $3.34^{8}$ & 3.367 & $3 \cdot 3^{96}$ & 3.405 & 3.425 & 3.444 & $3 \cdot 464$ & $3 \cdot 4^{84}$ & 3.5 & 3.525 \\
\hline 78 & 3.545 & 3.566 & $3 \cdot 5^{87}$ & 3.608 & 3.630 & 3.651 & 3.673 & 3.695 & 3.717 & 3.739 \\
\hline 79 & 3.762 & 3.785 & 3.808 & 3.831 & 3.854 & 3.878 & 3.902 & 3.926 & 3.950 & 3.975 \\
\hline 80 & 4.000 & 4.0 & 4.0 & 4.076 & 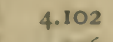 & 4.128 & .155 & 4.181 & 4.208 & $4.23^{6}$ \\
\hline 81 & 4.263 & 4.2 & 4.319 & $4 \cdot 34^{8}$ & 4.3 & 4.405 & 4.435 & 4.465 & 4. & 4.525 \\
\hline 82 & 4.556 & $4.5^{87}$ & 4.618 & 4.650 & 4.682 & 4.714 & 4.7 & 4.780 & 4.814 & 4.848 \\
\hline $8_{3}$ & 4.852 & 4.917 & 4.952 & 4.988 & 5.024 & $5.06 \mathrm{I}$ & 5.098 & 5.135 & 5.173 & 5.211 \\
\hline 84 & 5.250 & 5.289 & $5 \cdot 329$ & 5.369 & 5.410 & $5.45^{2}$ & 5.494 & 5.536 & 5.579 & 5.623 \\
\hline$\S_{5}$ & 5.667 & $5 \cdot 7$ & 5.7 & $5 . \mathrm{SO}_{3}$ & 5.849 & $=8$ & 5.944 & 5.493 & 6.042 & 6.092 \\
\hline 86 & 6.143 & 6.194 & 6 & 6 & 6.353 & 6. & & 6.519 & 6. & 6.634 \\
\hline 87 & 6.692 & $6.75^{2}$ & 6.813 & 6.874 & 6.937 & 7.000 & 7.065 & $7 \cdot 1.30$ & 7.197 & 7.264 \\
\hline s8 & $7 \cdot 333$ & 7.403 & $7 \cdot 475$ & $7 \cdot 547$ & 7.621 & $7 \cdot \ln (x) 6$ & $7.77^{2}$ & 7.850 & 7.029 & S.oxg \\
\hline 89 & 8.0511 & 8.174 & 8.259 & 8.346 & 8.434 & 8.524 & 8.615 & 8.709 & 8.504 & 8.001 \\
\hline 90 & 9.000 & 9.101 & 9.204 & 9.309 & 9.417 & 9.526 & $9.63^{8}$ & & 9.870 & 9.989 \\
\hline 91 & 10.11 & 10.33 & 10.36 & 10.49 & 10.63 & 10.77 & 10.90 & 11.05 & 11.20 & 11.35 \\
\hline 92 & 11.50 & 11.66 & 11.82 & $11 .(x)$ & 12.16 & 12.3 .3 & 12.51 & 12.70 & $12 \mathrm{Sig}$ & 13.08 \\
\hline 93 & 13.29 & 1.3 .49 & 13.71 & 13.93 & & 14. & 14.63 & 14.87 & & \\
\hline 94 & 15.67 & 15.25 & 16.24 & 16.54 & 16.86 & 17.18 & $17.5^{2}$ & 17.87 & 18.23 & 18.61 \\
\hline 95 & 19.00 & 19.41 & 19.83 & 20.28 & 20. & 21 & 2 & 22.26 & 22.81 & 23.39 \\
\hline 96 & 24.00 & 24.14 & $25 \cdot 32$ & 26.03 & 26.78 & 27.57 & 28.41 & 29. 30 & 30.25 & 31.26 \\
\hline 97 & 32.3 .3 & 3.4 .48 & 34.71 & 36.04 & $37 \cdot 46$ & 39.00 & 40.67 & 42.48 & 44.45 & 46.62 \\
\hline 98 & 4900 & 51.6 & 54.6 & 57.8 & 61.5 & 65.7 & 70.4 & 75.9 & 82.3 & 89.9 \\
\hline 99 & 990 & 110. & 124. & 142. & 866. & 199. & 249. & 332. & 499. & 999. \\
\hline
\end{tabular}




\title{
NATURAL SINES AND COSINES
}

\author{
TO
}

FOUR PLACES.

Note. For cosines use right-hand column of degrees and lower line of tenths.

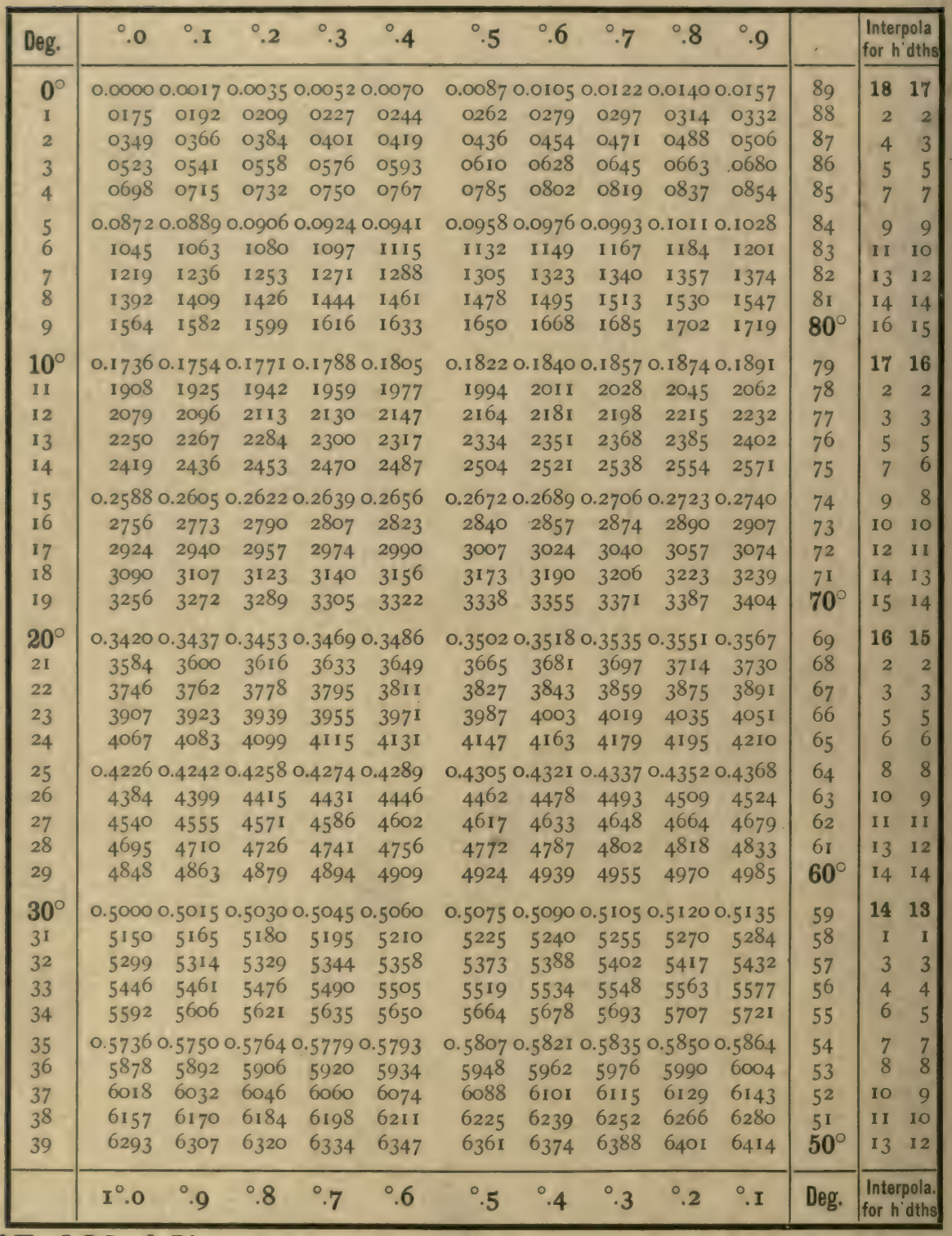

NAT. COS. 4 PL. 
4 PL. NAT. SIN.

\begin{tabular}{|c|c|c|c|c|c|c|c|c|c|c|c|c|c|}
\hline Deg. & $\stackrel{\circ}{\circ} 0$ & ${ }^{\circ} .1$ & ${ }^{\circ} .2$ & $\cdot 3$ & $\cdot 4$ & ${ }^{\circ} \cdot 5$ & .6 & ${ }^{\circ} \cdot 7$ & ${ }^{\circ} .8$ & $\cdot 9$ & & & \\
\hline 40 & 0.6428 & $0.6441 \mathrm{C}$ & .64550 & $0.6468 c$ & $0.648 I$ & $0.6494 \mathrm{C}$ & 0.6508 & 0.6521 & $0.6534 \mathrm{c}$ & 0.0547 & 49 & 13 & 12 \\
\hline 41 & 6561 & 6574 & $65^{87}$ & 6600 & 6613 & 6626 & 6639 & 6652 & 6665 & 6678 & 48 & I & I \\
\hline 42 & 6691 & 6704 & 6717 & 6730 & 6743 & 6756 & 6769 & 6752 & 6794 & 6807 & 47 & 3 & 2 \\
\hline 43 & 6820 & 6833 & 6845 & 6858 & 6871 & $688_{4}$ & 6896 & 6yog & 6921 & 6934 & 46 & 4 & 4 \\
\hline 44 & $69+7$ & 6959 & 6972 & $693_{4}$ & 6997 & 7009 & 7022 & 7034 & 7046 & 7059 & 45 & 5 & 5 \\
\hline 45 & 0.7071 & $0.7083 \mathrm{C}$ & $0.7096 \mathrm{c}$ & $0.7108 c$ & 0.7120 & 0.7133 & 0.7145 & $0.7^{1} 57$ & $0.7169 c$ & $0.718 \mathrm{I}$ & 44 & 7 & 6 \\
\hline 46 & 7193 & 7206 & 7218 & 7230 & 7242 & 7254 & 7266 & 7278 & 7290 & 7302 & 43 & 8 & 7 \\
\hline 47 & 7314 & 7325 & 7337 & 7349 & 7361 & 7373 & 7385 & 7396 & 7408 & 7420 & 42 & 9 & 8 \\
\hline 48 & 7431 & $7+43$ & 7455 & 7466 & 7478 & 7490 & 7501 & $75^{13}$ & $75^{24}$ & 7536 & 41 & 10 & 10 \\
\hline 49 & 7547 & 7559 & 7570 & $75^{81}$ & 7593 & 7604 & 7615 & 7627 & 7638 & 7649 & $40^{\circ}$ & 12 & 11 \\
\hline 50 & 0.7660 & $0.7672 \mathrm{c}$ & $.7683 c$ & 0.7694 & 0.7705 & 0.7716 & 0.7727 & $0.773^{8}$ & $0.7749 c$ & 0.7760 & 39 & 11 & 9 \\
\hline 51 & 7771 & 7782 & 7793 & 7804 & $7 \mathrm{~S} 15$ & 7826 & 7837 & 7848 & $75_{59}$ & 7869 & 38 & 1 & 1 \\
\hline $5^{2}$ & 7880 & 7891 & 7902 & 7912 & 7923 & 7934 & $79+4$ & 7955 & 7965 & 7976 & 37 & 2 & 2 \\
\hline 53 & $79 \$ 6$ & 7997 & 8007 & 8018 & 8028 & 8039 & 8049 & 8059 & 8070 & 8050 & 36 & 3 & 3 \\
\hline 54 & 8090 & 8100 & 8 III & 8121 & 8131 & $8 I_{4} \mathrm{I}$ & 8151 & 8161 & 8171 & 8181 & 35 & 4 & 4 \\
\hline 55 & 0.8192 & $0.8202 \mathrm{C}$ & .82110 & 0.8221 & 0.8231 & 0.8241 & 0.8251 & 0.8261 & $0.8271 \mathrm{C}$ & 0.8281 & 34 & 6 & 5 \\
\hline 56 & 8290 & 8300 & 8310 & 8320 & 8329 & 8339 & 8348 & $835^{8}$ & $8_{3} 68$ & 8377 & 33 & 7 & 5 \\
\hline 57 & $8_{3} 8_{7}$ & $8_{396}$ & 8406 & 8415 & 8425 & 8434 & 8443 & 8453 & 8462 & 8471 & 32 & 8 & 6 \\
\hline $5^{8}$ & 8480 & 8490 & 8499 & 8508 & 8517 & 8526 & 8536 & 8545 & 8554 & $8_{5} 6_{3}$ & 31 & 9 & 7 \\
\hline 59 & 8572 & $85^{81}$ & 8590 & 8599 & 8607 & 8616 & 8625 & 8634 & 8643 & 8652 & $30^{\circ}$ & 10 & 8 \\
\hline $60^{\circ}$ & 0.8660 & $0.8669 \mathrm{c}$ & $.8678 c$ & 0.8686 & 0.8695 & 0.8704 & 0.8712 & $0.872 \mathrm{I}$ & 0.8729 & $0.873^{8}$ & 29 & 8 & 7 \\
\hline 61 & 8746 & 8755 & 8763 & 8771 & 8780 & 8788 & 8796 & $880_{5}$ & 8813 & 8821 & 28 & 1 & 1 \\
\hline 62 & 8829 & $8_{3} 8$ & 8846 & 8854 & 8862 & 8870 & 8878 & 8886 & 8894 & 8902 & 27 & 2 & 1 \\
\hline 63 & 8910 & 8918 & 8926 & 8934 & 8942 & 8949 & 8957 & 8965 & 8973 & 8980 & 26 & 2 & 2 \\
\hline 64 & 8098 & 8996 & 9003 & 9011 & 9018 & 9026 & 9033 & 9041 & 9048 & 9056 & 25 & 3 & 3 \\
\hline 65 & $0.9063 c$ & $0.9070 \mathrm{C}$ & $0.9078 \mathrm{c}$ & $0.9085 \mathrm{c}$ & 0.9092 & 0.91000 & 0.9107 & 0.9114 & $0.9121 \mathrm{C}$ & 0.9128 & 24 & 4 & 4 \\
\hline 66 & 9135 & 9143 & 9150 & 9157 & 9164 & 9171 & 9178 & 9184 & 9191 & 9198 & 23 & 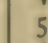 & 4 \\
\hline 67 & 9205 & 9212 & 9219 & 9225 & 9232 & 9239 & 9245 & 9252 & 9259 & 9265 & 22 & 6 & 5 \\
\hline 68 & 9272 & $927^{8}$ & 9285 & 9291 & 9298 & 9304 & 9311 & 9317 & 9323 & $933^{\circ}$ & 21 & 6 & 6 \\
\hline 69 & 9336 & $934^{2}$ & 9348 & 9354 & 9361 & 9367 & 9373 & 9379 & 9385 & 9391 & $20^{\circ}$ & 7 & 6 \\
\hline $70^{\circ}$ & 0.9397 & $0.9403 \mathrm{C}$ & .94090 & 0.94 & 0.94 & 0.9426 & 0.9432 & $0.943^{8}$ & $0.9444 C$ & 0.9449 & 19 & 6 & 4 \\
\hline 71 & 9455 & 9461 & 9466 & 9472 & 9478 & 9483 & $94 \$ 9$ & 9494 & 9500 & 9505 & 18 & I & o \\
\hline $7^{2}$ & 9511 & 9516 & 9521 & 9527 & 9532 & 9537 & $954^{2}$ & 9548 & 9553 & $955^{8}$ & 17 & 1 & I \\
\hline 73 & $95^{6} 3$ & 9568 & 9573 & 9578 & $95^{8} 3$ & 9588 & 9593 & 9598 & 9603 & 9608 & 16 & 2 & 1 \\
\hline 74 & 9613 & 9617 & 9622 & 9627 & 9632 & 9636 & 9641 & 9646 & 9650 & 9655 & 15 & 2 & 2 \\
\hline 75 & 0.9659 & 0.96640 & .96680 & $0.9673 \mathrm{C}$ & 0.9677 & $0.968 \mathrm{I}$ & 0.9686 & 0.9690 & $0.9694 c$ & 0.9699 & 14 & 3 & 2 \\
\hline $7^{6}$ & 9703 & 9707 & 9711 & 9715 & 9720 & 9724 & 9728 & 9732 & 9736 & 9740 & 13 & 4 & 2 \\
\hline 77 & 9744 & 9748 & 9751 & 9755 & 975 & 97634 & -9767 & 9770 & & $977^{8}$ & 12 & 4 & 3 \\
\hline 78 & $975 i$ & 9785 & 9789 & 9792 & 9796 & 9799 & $\int 9 \mathrm{SO}_{3}$ & $9 \$ 06$ & 9810 & 9813 & 11 & 5 & 3 \\
\hline 79 & 9816 & 9820 & 9823 & 9826 & 9829 & & $98_{36}$ & 9839 & $9 \delta_{42}$ & $9 S_{45}$ & $10^{\circ}$ & 5 & 4 \\
\hline 80 & $0.9848 c$ & 0.985 & $.98_{5}$ & 0.98 & 0.9860 & $0.9863 c$ & 0.9866 & 0.9869 & $0.9871 \mathrm{c}$ & 74 & 9 & 3 & 2 \\
\hline 81 & 9877 & gSSso & $98 S_{2}$ & $9 \mathrm{Ss}_{5}$ & $9 \$ 88$ & $98 y 0$ & 9893 & 9 Sos & $9 \sin 8$ & 9000 & 8 & o & o \\
\hline 82 & 9003 & 9005 & 9907 & 9010 & 9912 & 9914 & 9917 & 9919 & 9021 & 9) 23 & 7 & 1 & o \\
\hline $8_{3}$ & 9025 & 9928 & 99.30 & 9932 & 9934. & 9936 & 99,38 & 9940 & 9942 & 9943 & 6 & 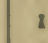 & 1 \\
\hline 84 & 9945 & 9947 & 9949 & 9951 & $995^{\circ}$ & 9954 & $995^{\circ}$ & 9957 & 9959 & 9060 & 5 & 8 & 1 \\
\hline$s_{5}$ & $0.9962 \mathrm{c}$ & 0.99630 & .99650 & $0.9966 \mathrm{c}$ & 0.9968 & $0.9969 c$ & 0.9971 & 0.9972 & $0.9973 \mathrm{C}$ & 0.9974 & 4 & 2 & I \\
\hline 86 & 9976 & 9977 & 9078 & 9979 & $99 \$ 0$ & 9081 & $99 \mathrm{~S}_{2}$ & $90 s_{3}$ & 9084 & 9085 & 3 & 2 & I \\
\hline 87 & 9986 & 0087 & 90,88 & 90.59 & 9900 & gropo & 9091 & 900,2 & gory 3 & 9093 & 2 & 2 & I \\
\hline 88 & 9904 & 9095 & 9995 & 9006 & 9906 & 9997 & yov7 & gov? & gros & 9008 & 1 & 2 & 2 \\
\hline \multirow[t]{2}{*}{89} & 9098 & youy & 9909 & 9999 & 9099 & 1.000 & 1.000 & 1.000 & 1.000 & 1.000 & $0^{\circ}$ & 3 & 2 \\
\hline & & & & & - & & & & & & 8. & \multicolumn{2}{|c|}{$\begin{array}{l}\text { Interpola. } \\
\text { for hi dths }\end{array}$} \\
\hline
\end{tabular}




\section{NATURAL TANGENTS AND COTANGENTS}

TO

FOUR PLACES.

Note. For cotangents use right-hand column of degrees and lower line of tenths

\begin{tabular}{|c|c|c|c|c|c|c|c|c|c|c|c|c|c|}
\hline Deg. & $\circ .0$ & $\circ \mathbf{I}$ & .2 & $\stackrel{\circ}{\cdot 3}$ & $\cdot 4$ & $\cdot 5$ & ${ }^{\circ} .6$ & $\cdot 7$ & ${ }^{\circ} .8$ & .9 & & \multicolumn{2}{|c|}{$\begin{array}{l}\text { Interpola. } \\
\text { for h'dths }\end{array}$} \\
\hline $0^{\circ}$ & \multicolumn{5}{|c|}{0.00000 .00170 .00350 .00520 .0070} & \multicolumn{5}{|c|}{0.00870 .01050 .01220 .01400 .0157} & 89 & 17 & 18 \\
\hline I & 0175 & OI92 & 0209 & 0227 & 0244 & 0262 & 0279 & 0297 & 0314 & 0332 & 88 & 2 & 2 \\
\hline 2 & 0349 & 0367 & 0384 & $\mathrm{O} 402$ & 0419 & 0437 & 0454 & 0472 & & 0507 & 87 & 3 & 4 \\
\hline 3 & 0524 & 0542 & 0559 & 0577 & 0594 & 0612 & 0629 & 0647 & 0664 & 0682 & 86 & 5 & 5 \\
\hline 4 & 0699 & 0717 & 0734 & $075^{2}$ & 0769 & 0787 & 0805 & 0822 & 0840 & 0857 & 85 & 7 & 7 \\
\hline 5 & \multicolumn{5}{|c|}{0.08750 .08920 .09100 .09280 .0945} & \multicolumn{5}{|c|}{0.09630 .09810 .09980 .10160 .1033} & 84 & 9 & 9 \\
\hline 6 & IO5I & 1069 & 1086 & I 104 & I 122 & I 139 & 1157 & 1175 & II 92 & 1210 & 83 & ro & II \\
\hline 7 & I 228 & 1246 & 1263 & I 28 I & 1299 & I317 & I 334 & 1352 & I 370 & 1388 & 82 & 12 & 13 \\
\hline 8 & 1405 & 1423 & 1441 & 1459 & 1477 & 1495 & 1512 & 1530 & I 548 & 1566 & 81 & 14 & 14 \\
\hline 9 & 1584 & 1602 & 1620 & 1638 & I655 & 1673 & 1691 & 1709 & 1727 & 1745 & $80^{\circ}$ & 15 & 16 \\
\hline $10^{\circ}$ & \multicolumn{5}{|c|}{0.17630 .17810 .17990 .18170 .1835} & \multicolumn{5}{|c|}{0.18530 .18710 .18900 .19080 .1926} & 79 & 19 & 20 \\
\hline II & 1944 & I $(1) 2$ & 1980 & 1998 & 2016 & 2035 & 2053 & 2071 & 2089 & 2107 & 78 & 2 & 2 \\
\hline 12 & 2126 & 2144 & 2162 & 2180 & 2199 & 2217 & 2235 & & & 2290 & 77 & 4 & \\
\hline 13 & 2309 & 2327 & 2345 & 2364 & 2382 & 2401 & 2419 & 2438 & 2456 & 2475 & 76 & 6 & 6 \\
\hline 14 & 2493 & 2512 & 2530 & 2549 & 2568 & 2586 & 2605 & 2623 & 2642 & 2661 & 75 & 8 & 8 \\
\hline 15 & \multicolumn{5}{|c|}{0.26790 .26980 .27170 .27360 .2754} & \multicolumn{5}{|c|}{0.27730 .27920 .28 I 10.28300 .2849} & 74 & 10 & 10 \\
\hline 16 & 2867 & 2886 & 2905 & 2924 & 2943 & 2962 & 2981 & 3000 & 3019 & 2038 & 73 & II & 12 \\
\hline 17 & 3057 & 3076 & 3096 & $31: 5$ & 3134 & 3153 & $3^{I}$ & & & 3230 & 72 & 13 & 14 \\
\hline 18 & 3249 & 3269 & 3288 & 3307 & 33 & 3346 & 3365 & 3385 & 3404 & 3424 & 71 & 15 & 16 \\
\hline 19 & 3443 & 3463 & 3482 & 3502 & 35 & 3541 & $35^{61}$ & $35^{81}$ & 3600 & 3620 & $70^{\circ}$ & 17 & 18 \\
\hline $20^{\circ}$ & \multicolumn{5}{|c|}{0.36400 .36590 .36790 .36990 .3719} & \multicolumn{5}{|c|}{0.37390 .37590 .37790 .37990 .3819} & 9 & \multicolumn{2}{|c|}{2224} \\
\hline 21 & 3839 & 3859 & $3^{879}$ & 3899 & 3919 & 3939 & 3959 & 3979 & 4000 & 4020 & 8 & 2 & 2 \\
\hline 22 & 4040 & 4061 & 4081 & $4 \mathrm{rOI}$ & 4122 & 41 & 41 & $4^{1}$ & 4 & 4224 & 7 & 4 & 5 \\
\hline 23 & 4245 & 4265 & 4286 & 4307 & 43 & 4348 & 4369 & 4390 & & 4431 & 66 & 7 & 7 \\
\hline 24 & $445^{2}$ & 4473 & 4494 & 4515 & 4536 & 4557 & 4578 & 4599 & 4621 & 4642 & 65 & 9 & 10 \\
\hline 25 & \multicolumn{5}{|c|}{0.46630 .46840 .47060 .47270 .4748} & \multicolumn{5}{|c|}{0.47700 .47910 .48130 .48340 .4856} & 64 & II & 12 \\
\hline 26 & 4877 & 4899 & 4921 & $494^{2}$ & 4964 & 4986 & 5008 & 5029 & 5051 & 5073 & 63 & I3 & 14 \\
\hline 27 & 5095 & 5117 & $5^{139}$ & 5161 & 518 & 5206 & 5228 & & & 5295 & 62 & 15 & 17 \\
\hline 28 & 5317 & 5340 & 5362 & 5384 & 5407 & 5430 & $545^{2}$ & 5475 & 5498 & 5520. & $6 I$ & 18 & 19 \\
\hline 29 & 5543 & 5566 & $55^{89}$ & 5612 & $5^{6} 35$ & $565^{8}$ & 5681 & 5704 & 5727 & $575^{\circ}$ & $60^{\circ}$ & 20 & 22 \\
\hline $30^{\circ}$ & \multicolumn{5}{|c|}{$0.57740 .57970 .5^{820} 0.5^{844} 0.5^{867}$} & \multicolumn{5}{|c|}{$0.5^{890} 0.59140 .59380 .59610 .5985$} & 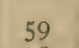 & 26 & 28 \\
\hline 31 & 6009 & 6032 & 6056 & 6080 & 6104 & 6128 & $615^{2}$ & 6176 & 6200 & 6224 & $5^{8}$ & 3 & 3 \\
\hline 32 & 6249 & 6273 & 6297 & 6322 & 6346 & $637 x$ & 6395 & 64 & 6445 & 6469 & 57 & 6 & 6 \\
\hline 33 & 6494 & 6519 & 6544 & 6569 & 659 & 6619 & 6644 & 6669 & 6694 & 6720 & $5^{6}$ & 8 & 8 \\
\hline 34 & 6745 & 6771 & 6796 & 6822 & 6847 & 6873 & 6899 & 6924 & $695^{\circ}$ & 6976 & 55 & 10 & II \\
\hline 35 & \multicolumn{5}{|c|}{0.70020 .70280 .70540 .70800 .7107} & \multicolumn{5}{|c|}{0.71330 .71590 .71860 .72120 .7239} & 54 & 13 & 14 \\
\hline 36 & 7265 & 7292 & 7319 & 7346 & 7373 & 7400 & 7427 & 7454 & 7481 & 7508 & 53 & 16 & 17 \\
\hline 37 & 7536 & $75^{6} 3$ & 759 & 7618 & & 7673 & & & 7757 & 7785 & $5^{2}$ & 18 & 20 \\
\hline 30 & 7813 & 7841 & 7869 & 7898 & 792 & & 7983 & 8012 & 8040 & 8069 & 51 & 21 & 22 \\
\hline \multirow[t]{2}{*}{39} & 8098 & 8127 & 8156 & 8185 & 8214 & 8243 & 8273 & 8302 & 8332 & 8361 & $50^{\circ}$ & 23 & 25 \\
\hline & $I^{\circ}$ & & & & & & & & ${ }^{\circ} .2$ & & Deg. & \multicolumn{2}{|c|}{$\begin{array}{l}\text { Interpola. } \\
\text { for h'dths }\end{array}$} \\
\hline
\end{tabular}

NAT. COT. 4 PL. 
NATURAL TANGENTS AND COTANGENTS.

4 PL. NAT. TAN.

\begin{tabular}{|c|c|c|c|c|c|c|c|c|c|c|c|c|}
\hline Deg. & ${ }^{\circ} .0$ & $\stackrel{\circ}{\circ}$ & $\stackrel{\circ}{\circ}$ & ${ }^{\circ} \cdot 3$ & ${ }^{\circ} \cdot 4$ & ${ }^{\circ} .5$ & ${ }^{\circ} .6$ & ${ }^{\circ} \cdot 7$ & .8 & ${ }^{\circ} .9$ & & $\begin{array}{l}\text { Interpola. } \\
\text { for h dths }\end{array}$ \\
\hline $40^{\circ}$ & $0.8391 \mathrm{C}$ &.$\S_{4210}$ & $0.845^{1}$ & $0 . S_{4} S_{1}$ & 0.8511 & $0 . \$_{541}$ & $0.8571 \mathrm{C}$ & 0.86010 & $0.8632 \mathrm{c}$ & 0.8662 & 49 & 3040 \\
\hline 41 & 8693 & 8724 & $S_{754}$ & $8_{7} s_{5}$ & 8816 & 8847 & 8878 & 8910 & 8941 & 8972 & 48 & 34 \\
\hline 42 & 9004 & 9036 & 9067 & 9099 & 9131 & 9163 & 9195 & 9228 & 9260 & 9293 & 47 & 68 \\
\hline 43 & 9325 & $935^{8}$ & 9391 & 9424 & 9457 & 9490 & 9523 & 9556 & 9590 & 9623 & 46 & 912 \\
\hline 44 & 9657 & 9691 & 9725 & 9759 & 9793 & 9827 & $986 \mathrm{I}$ & 9896 & 9930 & 9965 & 45 & 1216 \\
\hline 45 & 1.00001 & $1.0035 \mathrm{I}$ & 1.0070 & 1.0105 & 1.0141 & $1.017^{6}$ & 1.0212 & $1.0247 \mathrm{I}$ & $1.028_{3} I$ & 1.0319 & 44 & 1520 \\
\hline 46 & o355 & 0392 & 0428 & 0464 & 0501 & 0538 & 0575 & 0612 & 0649 & $06 \$ 6$ & 43 & 1824 \\
\hline 47 & 0724 & 0761 & 0799 & 0837 & 0875 & 0913 & 0951 & 0990 & 1028 & 1067 & 42 & 2128 \\
\hline $4^{8}$ & 1106 & 1145 & 1184 & 1224 & 1263 & 1303 & 1343 & ${ }_{13} \delta_{3}$ & 1423 & 1463 & 41 & $243^{2}$ \\
\hline 49 & 1504 & 1544 & $15^{8} 5$ & 1626 & 1667 & 1708 & 1750 & 1792 & 1833 & 1875 & $40^{\circ}$ & 2736 \\
\hline $50^{\circ}$ & 1.19181 & $1.1960 \mathrm{I}$ & 1.2002 & 1.2045 & 1.2088 & 1.2131 & 1.2174 & $1.2218 \mathrm{I}$ & 1.22611 & 1.2305 & 39 & 5060 \\
\hline $5^{I}$ & 2349 & 2393 & 2437 & 2482 & 2527 & $257^{2}$ & 2617 & 2662 & 2708 & 2753 & 38 & 56 \\
\hline $5^{2}$ & 2799 & 2546 & 2892 & $293^{8}$ & $298_{5}$ & 3032 & 3079 & $3^{127}$ & 3175 & 3222 & 37 & 1012 \\
\hline 53 & 3270 & 3319 & 3367 & 3416 & 3465 & $35^{14}$ & 3564 & $3^{613}$ & $366_{3}$ & $37^{13}$. & 36 & 1518 \\
\hline 54 & 3764 & 3814 & 3865 & 3916 & 3968 & 4019 & 4071 & 4124 & 4176 & 4229 & 35 & 2024 \\
\hline & 1.42811 & $.4335 \mathrm{I}$ & $1.43^{88}$ & $1.444^{2}$ & I. 4496 & $1.455^{\circ}$ & 1.4605 & 1.4659 & 1.4715 & 1.4770 & 34 & 2530 \\
\hline 56 & 4826 & 4882 & $493^{8}$ & 4994 & 5051 & 5108 & 5166 & 5224 & $52 \delta_{2}$ & 5340 & 33 & 3036 \\
\hline 57 & 5399 & $545^{8}$ & $55^{17}$ & 5577 & 5637 & 5697 & 5757 & $5^{818}$ & 5880 & 5941 & 32 & 3542 \\
\hline $5^{8}$ & 6003 & 6066 & 6128 & 6191 & 6255 & 6319 & $6_{3} 8_{3}$ & 6447 & 6512 & 6577 & 31 & 4048 \\
\hline 59 & 6643 & 6709 & 6775 & 6842 & 6909 & 6977 & 7045 & 7113 & 7182 & 7251 & $30^{\circ}$ & 4554 \\
\hline $60^{\circ}$ & 1.73211 & & $1.746 \mathrm{I}$ & $1.753^{2}$ & 1.7603 & 1.7675 & I & 1.7820 & 1.7893 & 1.7966 & 29 & 7080 \\
\hline UI & 8040 & 8115 & 8190 & 8265 & 8341 & 8418 & 8495 & 8572 & 8650 & 8728 & 28 & 78 \\
\hline 62 & 8807 & 8887 & 8967 & 9047 & 9128 & 9210 & 9292 & 9375 & $945^{8}$ & 9542 & 27 & 1416 \\
\hline 63 & $1.9626 \mathrm{I}$ & 1.97111 & 1.9797 & $1.988_{3}$ & I.9970 & 2.0057 & $2.0145=$ & 2.0233 & 2.03232 & 2.0413 & 26 & 2124 \\
\hline 64 & 2.05032 & 2.05942 & & 2.07 & $2.087^{2}$ & 2.0965 & 2.1060 & 2.1155 & 2.12512 & 2.1348 & 25 & 2832 \\
\hline 65 & 2.14452 & 2.15432 & 2.1642 & 2.1742 & 2.1842 & 2.19432 & 2.2045 & $2.2148=$ & 2.22512 & 2.2355 & 24 & 3540 \\
\hline 66 & 2460 & 2566 & 2673 & 2781 & 2889 & 2998 & $3^{109}$ & 3220 & 3332 & 3445 & 23 & $4^{2} 48$ \\
\hline 67 & 3559 & 3673 & 3789 & 3906 & 4023 & 4142 & 4262 & $43_{3} 8_{3}$ & 4504 & 4627 & 22 & 4956 \\
\hline 68 & $475^{1}$ & 4876 & 5002 & $5^{129}$ & 5257 & $53^{86}$ & $55^{17}$ & 5649 & 5782 & 5916 & 21 & $56 \quad 64$ \\
\hline 69 & 6051 & 6187 & 6325 & 6464 & 6605 & 6746 & 6889 & 7034 & 7179 & 7326 & 20 & 6372 \\
\hline $70^{\circ}$ & $2.7475^{2}$ & 2.76252 & 2.7776 & 2.79 & $2.808_{3}$ & 2.8239 & 2 & 2.8556 & 2.8716 & $2.88 ; 8$ & 19 & 90 \\
\hline $7^{1}$ & $2.9042=$ & 292082 & 2.9375 & 2.9544 & 2.9714 & 2.9887 & 3.0061 & 3.0237 & 3.0415 & 3.0595 & 18 & 9 \\
\hline $7^{2}$ & 3.07773 & 3.00613 & 3.1146 & 3.1334 & $3.15^{24}$ & 3.17163 & 3.1910 & 3.2106 & 3.2305 & 3.2506 & 17 & 18 \\
\hline 73 & 2709 & 2914 & 3122 & 3332 & 3544 & 3759 & 3977 & 4197 & $44^{20}$ & 4646 & 16 & 27 \\
\hline 74 & $4 S_{74}$ & 5105 & 5339 & 5576 & 5816 & 6059 & 6305 & 6554 & 6806 & 7062 & 15 & 36 \\
\hline 75 & 3.73213 & $3.75^{8} 3$ & 3.7848 & 3.8118 & 3.8391 & 3.86673 & 3.8947 & $3.923^{2}$ & 3.9520 & 3.9812 & 14 & 45 \\
\hline 76 & 4.01084 & 1.04 & 4.0 & 4.1022 & 4.1335 & $4.16_{53}$ & 4.19 & 4 & 4.26 & 4.2972 & 13 & 54 \\
\hline 77 & 4.33154 & 4.36624 & $4 \cdot 4 c$ & 4.4 .374 & $4 \cdot 4737$ & 4.51074 & $4.54{ }^{8} 3$ & $4.5^{864}$ & 4.6252 & 4.6046 & 12 & 63 \\
\hline $7^{8}$ & 4.70464 & .74534 & 4.7867 & 4.8288 & 4.8716 & 4.91524 & 4.9594 & 5.0 & 5.0504 & 5.0970 & 11 & 72 \\
\hline 79 & $5.144^{6} 5$ & 5.19295 & 5.24225 & 5.25 & 5.3435 & 5.39555 & $5 \cdot 4$ & 8 & $5.557^{8}$ & 5.6140 & 10 & 81 \\
\hline $80^{\circ}$ & 5.67135 & 5.7297 & 5.7894 & 5.8502 & 5.9124 & $5.975^{8}$ & 6.0405 & 6.1066 & 6.1742 & 6.243 & 9 & \\
\hline 81 & 6.31380 & $3^{3} 5_{90}$ & 6.45 & 6.5350 & 6.6122 & 6.6912 & 6.77 & $6 . S_{5}$ & 6.9395 & 7.0264 & 8 & \\
\hline $8_{2}$ & 7.11547 & .20667 & $7 \cdot 3002$ & $7 \cdot 3962$ & 7.4047 & $7.595^{8}$ & 7.6996 & 7.5062 & $7.915^{5}$ & $8.02 S_{5}$ & 7 & \\
\hline 83 & 8.1443 & $3.26,368$ & $8.3 \mathrm{~S}_{3}$ & 8.5126 & 8.6427 & 8. $77^{(x)}$ & 8.9152 & 9.0579 & 9.2052 & $9.357^{2}$ & 6 & \\
\hline 84 & 9.5144 & 9.677 & 9.845 & 10.02 & 10.20 & 10.39 & 10.58 & $10.7^{8}$ & 10.09 & 11.20 & 5 & \\
\hline $8_{5}$ & 11.43 & 11.66 & 11.98 & 12.16 & 12.43 & 12.71 & 13.00 & 13.30 & 13.62 & 13.95 & 4 & \\
\hline 86 & 14.30 & 14.67 & 15.06 & $15.4^{6}$ & 15.89 & 16.35 & $16 . s_{3}$ & 17.34 & 17.89 & 18.46 & 3 & \\
\hline 87 & 19.08 & 19.74 & 20.45 & 21.20 & 22.02 & 22.90 & 23.80 & 2490 & 26.03 & 27.27 & 2 & \\
\hline 88 & 28.64 & 30.14 & 31.82 & $33 \cdot(x)$ & 35.50 & $3^{5.19}$ & 40.92 & 44.07 & 47.74 & 52.08 & 1 & \\
\hline \multirow[t]{2}{*}{89} & 57.20 & 63.663 & 71.62 & 81.85 & 95.49 & 114.6 & 143.2 & 191.0 & 286.5 & 573.0 & 0 & \\
\hline & $I^{\circ} \cdot 0$ & & & & . & & & & .2 & 11 & Deg. & $\begin{array}{l}\text { Interpola. } \\
\text { for h dths }\end{array}$ \\
\hline
\end{tabular}




\section{LOGARITHMS OF SINES AND COSINES}

TO

\section{FOUR PLACES.}

Note. For log. cos. use right-hand column of degrees and lower line of tenths.

\begin{tabular}{|c|c|c|c|c|c|c|c|c|c|c|c|c|c|}
\hline Deg. & $\circ 0$ & . I & ${ }^{\circ} .2$ & $\cdot 3$ & $\cdot 4$ & $\cdot 5$ & ${ }^{\circ} .6$ & $\cdot 7$ & $\circ 8$ & 99 & & \multicolumn{2}{|c|}{$\begin{array}{l}\text { Interpola. } \\
\text { for h'dths }\end{array}$} \\
\hline $0^{\circ}$ & \multicolumn{5}{|c|}{${ }_{-\infty} \overline{\underline{3}} \cdot 2419 \overline{\overline{3}} \cdot 5429 \overline{\overline{3}} \cdot 7190 \overline{\overline{3}} \cdot 8439$} & \multicolumn{5}{|c|}{$\overline{3} .9408 \overline{2} .0200 \overline{2} .0870 \overline{2} .1450 \overline{2} .1961$} & oy & 35 & \\
\hline I & \multirow{2}{*}{\multicolumn{5}{|c|}{$\begin{array}{l}\overline{2} .2419 \overline{2} .2832 \overline{2} .3210 \overline{2} .355^{8} \overline{2} .3880 \\
\overline{2} .5428 \overline{2} .5640 \overline{2} .5842 \overline{2} .6035 \\
\overline{2} .6220\end{array}$}} & \multicolumn{5}{|c|}{$\overline{2} .4179 \overline{2} .4459 \overline{2} .4723 \overline{2} .4971 \overline{2} .5206$} & 88 & 4 & \\
\hline 2 & & & & & & \multirow{2}{*}{\multicolumn{5}{|c|}{ 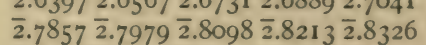 }} & 37 & 7 & \\
\hline 3 & \multirow{2}{*}{\multicolumn{5}{|c|}{ 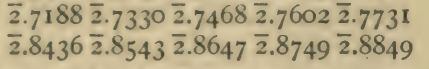 }} & & & & & & 86 & II & \\
\hline 4 & & & & & & \multirow{2}{*}{\multicolumn{5}{|c|}{$\begin{array}{l}2.89462 .90422 .91352 .92262 .9315 \\
\overline{2} .0816 \overline{2} .9804 \overline{2} .9070 \bar{J}\end{array}$}} & 85 & 14 & ( \\
\hline 5 & \multicolumn{5}{|c|}{$\overline{\mathbf{z}} .9403^{\overline{2}} .9489 \overline{\mathbf{z}} .9573^{\overline{2} .96} 55^{\overline{2} .9736}$} & & & & & & 84 & 8 & 13 \\
\hline 6 & \multicolumn{2}{|c|}{$\overline{1} .0192 \bar{I} .0264$} & & - & I.0472 & \multicolumn{5}{|c|}{$\overline{\mathrm{I}} .0539 \overline{\mathrm{I}} .0605$ I.0670 $\overline{\mathrm{I}} .0734 \overline{\mathrm{I}} .0797$} & & 21 & 15 \\
\hline 7 & \multirow{2}{*}{\multicolumn{2}{|c|}{$\overline{\mathrm{I}} .0859 \overline{\mathrm{I}} .0920$}} & I. & $\overline{\mathbf{I}}$. & 9 & \multicolumn{5}{|c|}{$\overline{\mathrm{I}} .1157 \overline{\mathrm{I}} .1214 \overline{\mathrm{I}} .127 \mathrm{I} \overline{\mathrm{I}} .1326 \overline{\mathrm{I}} .138 \mathrm{I}$} & 82 & 5 & 18 \\
\hline 8 & & & $\overline{\mathbf{i}}$. & $\overline{\mathbf{I}}$. & 6 & \multicolumn{5}{|c|}{ 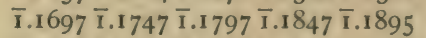 } & 81 & 0 & 20 \\
\hline 9 & \multicolumn{2}{|c|}{$\frac{1.14301 .1489}{1.1943 \overline{1} .1991}$} & & 1.20 & I & $\overline{\mathrm{I}} .2176 \overline{\mathrm{I}}$ & & & & & $80^{\circ}$ & 32 & 23 \\
\hline $10^{\circ}$ & \multicolumn{5}{|c|}{$\overline{\mathrm{I}} .2397 \overline{\mathrm{I}} .2439 \overline{\mathrm{I}} .2482 \overline{\mathrm{I}} .2524 \overline{\mathrm{I}} .2565$} & \multicolumn{5}{|c|}{$\overline{\mathrm{I}} .2606 \overline{\mathrm{I}} .2647 \overline{\mathrm{I}} .2687 \overline{\mathrm{I}} .2727 \overline{\mathrm{I}} .2767$} & 9 & 21 & 20 \\
\hline I & & & & & 2959 & 2997 & 3034 & 3070 & 3107 & 3143 & 8 & 2 & 2 \\
\hline $\mathbf{I}$ & 3 & & & & & & & & & & 77 & 4 & 4 \\
\hline I & & 3 & & & & & & & & ( & & 6 & J \\
\hline 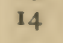 & 3837 & & & 3927 & 3957 & 3900 & $4=-3$ & T & $40 / 3$ & 4102 & 75 & 8 & 7 \\
\hline 15 & \multicolumn{5}{|c|}{$\overline{\mathrm{I}} .4 \mathrm{I} 30 \overline{\mathrm{I}} .4 \mathrm{I} 58 \overline{\mathrm{I}} .4 \mathrm{I} 86 \overline{\mathrm{I}} .42 \mathrm{I} 4 \mathrm{\textrm {I }} .4242$} & \multicolumn{5}{|c|}{$\overline{\mathrm{I}} .4269 \overline{\mathrm{I}} .4296 \overline{\mathrm{I}} .4323 \overline{\mathrm{I}} .4350 \overline{\mathrm{I}} .4377$} & 74 & I & 9 \\
\hline 1 & 4403 & 4430 & $445^{6}$ & 4482 & 4508 & 4533 & & $45^{84}$ & 4609 & 4634 & 73 & 3 & 11 \\
\hline 1 & & & & & & & & & & 4876 & 72 & 5 & I 3 \\
\hline $\mathbf{I}$ & & & & & & & & & & 4 & 71 & 7 & 14 \\
\hline 19 & 5126 & 514 & & 5 & & & & 5278 & 5299 & 5320 & $70^{\circ}$ & 19 & 16 \\
\hline $20^{\circ}$ & \multicolumn{5}{|c|}{$\overline{\mathbf{I}} .534 \mathrm{I} \overline{\mathbf{I}} .536 \mathrm{I} \overline{\mathrm{I}} .53^{82} \overline{\mathrm{I}} \cdot 5402 \overline{\mathbf{I}} .5423$} & \multicolumn{5}{|c|}{$\overline{\mathbf{I}} .5443 \overline{\mathrm{I}} \cdot 5463 \overline{\mathrm{I}} \cdot 5484 \overline{\mathrm{I}} \cdot 5504 \overline{\mathrm{I}} .5523$} & 69 & 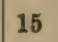 & 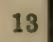 \\
\hline 2 & & & & & & & & & & & 8 & 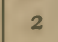 & $\theta^{2}$ \\
\hline 2 & & & & & & & & & & & 7 & 3 & \\
\hline 2 & & & & & & & & & & 6076 & 66 & & \\
\hline 24 & & & & & & & & & & & $u_{5}$ & & 3 \\
\hline 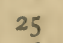 & $\overline{\mathbf{1}} .6259^{\overline{\mathrm{T}}}$ & $\overline{1} .6276 \bar{i}$ & $\bar{I} .6292 \bar{I}$ & $\overline{1} .6308$ & $\overline{\mathrm{I}} .6324$ & $\overline{\mathbf{I}}$ & & & & 3 & 64 & 8 & \\
\hline 2 & & & & 6465 & 6480 & & & & 654 & 6556 & 63 & $y$ & \\
\hline 2 & & & & 6615 & & 66 & & & & 67 & 62 & II & 9 \\
\hline 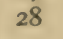 & & & & & $6 \%$ & 6787 & 68 & 68 & 6828 & 6842 & 61 & 12 & 10 \\
\hline 29 & & 686 & & & 6910 & 60 & 6 & & 6963 & 6977 & $60^{\circ}$ & 14 & 12 \\
\hline 30 & $\overline{\mathbf{1}} .6$ & & & & & & & & & 106 & & $=$ & 9 \\
\hline $3 \mathbf{I}$ & & & & & & 7 & & & & 7230 & $5^{8}$ & 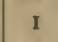 & 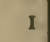 \\
\hline 32 & & & & & & & & & & 7349 & 57 & & s \\
\hline 33 & & & 7 & 7 & & & & & & 7464 & 56 & & \\
\hline 34 & 7476 & 7487 & 7498 & 7509 & & (100 & דיד & 年 & . & 7575 & 55 & & 4 \\
\hline 35 & $\overline{\mathrm{x}} .75^{86}$ & $\overline{\mathbf{I}} \cdot 759^{\prime}$ & $\overline{1} .760$ & $\bar{I} .7618$ & I. 7629 & $\overline{\mathbf{I}} \cdot 7640$ & I. 76 & $\overline{\mathrm{I}} \cdot 766 \mathrm{I}$ & $\overline{1} .7671$ & I. 7682 & 54 & 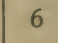 & 5 \\
\hline on & -60 & 77 & & & 77 & & & 7764 & 7774 & 7785 & 53 & 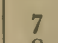 & \\
\hline 37 & & & & & & & & & & & & & \\
\hline 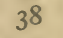 & & 79 & & & & & & & & & & 9 & \\
\hline 39 & & & & 8017 & 80 & 8035 & & 8053 & & 80 & $50^{\circ}$ & 10 & 0 \\
\hline & & & & & & & & & & & & & \\
\hline
\end{tabular}

LOG. COS. 4 PL. 
4 PL. LOG. SIN.

\begin{tabular}{|c|c|c|c|c|c|c|c|c|c|c|c|c|}
\hline Deg. & ${ }^{\circ} .0$ & ${ }^{\circ} .1$ & ${ }^{\circ} .2$ & $\cdot 3$ & ${ }^{\circ} .4$ & ${ }^{\circ} \cdot 5$ & ${ }^{\circ} .6$ & ${ }^{\circ} \cdot 7$ & $\circ 8$ & .9 & & $\begin{array}{c}\text { Interpola. } \\
\text { tables. }\end{array}$ \\
\hline $40^{\circ}$ & $\overline{1} \cdot \operatorname{Sos}_{1}$ & I. 8090 & İ.8099 & $\overline{1} .8108$ & 1.8117 & I. $8125 \overline{1}$ & 1.8134 & I. 8143 & $8.8152 \bar{I}$ & $\overline{1} .816 \mathrm{I}$ & 49 & 987 \\
\hline 41 & 8169 & 8178 & 8187 & 8195 & 8204 & 8213 & 8221 & 8230 & 8238 & 8247 & 48 & $\begin{array}{lll}1 & 1 & 1\end{array}$ \\
\hline 42 & 8255 & 8264 & 8272 & 8280 & 8289 & 8297 & $830_{5}$ & $8_{313}$ & $8_{322}$ & $8_{330}$ & 47 & 221 \\
\hline 43 & $833^{8}$ & 8346 & 8354 & 8362 & 8370 & 8378 & 8386 & 8394 & 8402 & 8410 & 46 & $\begin{array}{ll}322 \\
\end{array}$ \\
\hline 44 & 8418 & 8426 & 8433 & 8441 & 8449 & 8457 & 8464 & $847^{2}$ & 8480 & 8487 & 45 & 433 \\
\hline 45 & T. $\$ 495$ & 1.8502 & $\overline{\mathrm{I}} .8510$ & 1.8517 & I. 8525 & I. 8532 I & I. $8540 \mathrm{I}$ & I. 8547 & $.8555^{\mathrm{I}}$ & 1.8562 & 44 & 544 \\
\hline 46 & $8_{5} 69$ & $8_{577}$ & 8584 & 8591 & 8598 & 8606 & 8613 & 8620 & 8627 & 8634 & 43 & 554 \\
\hline 47 & 8641 & 8648 & 8655 & 8662 & 8669 & 8676 & 8683 & 8690 & 8697 & 8704 & 42 & 665 \\
\hline 48 & 8711 & $8_{718}$ & 8724 & 8731 & $873^{8}$ & 8745 & $875^{1}$ & $875^{8}$ & 8765 & 8771 & $4 I$ & 766 \\
\hline 49 & 8778 & 8784 & 8791 & 8797 & 8804 & 8810 & 8817 & 8823 & 8830 & 8836 & $40^{\circ}$ & 876 \\
\hline $50^{\circ}$ & $\overline{\mathrm{I}} .88+3$ & 1.8849 & $\overline{1} .8855$ & 1.8862 & I. 8868 & $\overline{\mathrm{I}} .8874 \mathrm{I}$ & 1.8880 i & 1.8887 & $.8893 \overline{\mathrm{I}}$ & і. .8899 & 39 & 654 \\
\hline 51 & $8 y 05$ & 8911 & 8917 & 8923 & 8929 & 8935 & $89+1$ & 8947 & 8953 & 8959 & 38 & 110 \\
\hline 52 & 8965 & 8971 & 8977 & $898_{3}$ & 8989 & 8995 & 9000 & 9006 & 9012 & 9018 & 37 & $\begin{array}{lll}1 & 1 & 1 \\
2 & & \end{array}$ \\
\hline 53 & 9023 & 9029 & 9035 & 9041 & 9046 & 9052 & 9057 & 9063 & 9069 & 9074 & 36 & $\begin{array}{lll}2 & 2 & 1\end{array}$ \\
\hline 54 & $90 S 0$ & $90 S_{5}$ & 909 I & 9096 & 9101 & 9107 & 9112 & 9118 & 9123 & 9128 & 35 & 222 \\
\hline 55 & $\overline{\mathrm{I}} 9134 \overline{1}$ & 1.9139 & I. 9144 & $1.9149^{i}$ & I.9155 & $\overline{1} .9160 \overline{1}$ & $1.9165 \overline{1}$ & 1.91701 & $.9175 \overline{\mathrm{I}}$ & $.918 \mathrm{I}$ & 34 & 332 \\
\hline 56 & 9186 & 9191 & 9196 & 9201 & 9206 & 9211 & 9216 & 9221 & 9226 & 9231 & 33 & 432 \\
\hline 57 & 9236 & 9241 & 9246 & 9251 & 9255 & 9260 & 9265 & 9270 & 9275 & 9279 & 32 & 443 \\
\hline $5^{8}$ & 9284 & 9289 & 9294 & 9298 & 9303 & 9308 & 9312 & 9317 & 9322 & 9326 & $3 I$ & 543 \\
\hline 59 & 9331 & 9335 & 9340 & 9344 & 9349 & 9353 & $935^{8}$ & 9362 & 9367 & 9371 & $30^{\circ}$ & 554 \\
\hline $60^{\circ}$ & T.9375 & $1.9380 \overline{1}$ & I. 9384 & I. 9388 & $\overline{1} .9393$ & I. $.9397 \bar{I}$ & $.9401 \mathrm{I}$ & $.9406 \bar{I}$ & $.9410 \overline{1}$ & .9414 & 29 & 432 \\
\hline 61 & 9418 & 9422 & 9427 & $943 \mathrm{I}$ & 9435 & 9439 & 9443 & 9447 & $945^{1}$ & 9455 & 28 & 000 \\
\hline 62 & 9459 & 9463 & 9467 & 9471 & 9475 & 9479 & 9483 & 9487 & 949 I & 9495 & 27 & 110 \\
\hline 63 & 9499 & 9503 & 9506 & 9510 & $9514^{\circ}$ & 9518 & 9522 & 9525 & 9529 & 9533 & 26 & 111 \\
\hline 64 & 9537 & $954^{\circ}$ & 9544 & $954^{8}$ & $955^{I}$ & 9555 & $955^{8}$ & 9562 & 9566 & 9569 & 25 & 211 \\
\hline 65 & $\overline{1} .9573 \bar{I}$ & $9576 \mathrm{i}$ & I. 9580 & .9583 & I. $95^{87}$ & I. 9590 i & I. $9594 \bar{I}$ & .9597 I & $.9601 \overline{1}$ & 1.9604 & 24 & 221 \\
\hline 66 & 9607 & 9611 & 9614 & 9617 & 9621 & 9624 & 9627 & 9631 & 9634 & 9637 & 23 & 221 \\
\hline 67 & 9640 & 9643 & 9647 & 9650 & 9653 & 9656 & 9659 & 9662 & 9666 & 9669 & 22 & $\begin{array}{lll}3 & 2 & 1\end{array}$ \\
\hline 68 & 9672 & $9^{6} 75$ & 9678 & 9681 & 9684 & 9687 & 9690 & 9693 & 9696 & 9699 & 21 & 322 \\
\hline 69 & 9702 & 9704 & 9707 & 9710 & $97^{13}$ & 9716 & 9719 & 9722 & 9724 & 9727 & $20^{\circ}$ & 432 \\
\hline $70^{3}$ & $\overline{\mathbf{I}} .973 \mathrm{O} \overline{\mathrm{I}}$ & 1.9733 & i. $9735^{i}$ & i. $973^{\circ}$ & $\overline{1} .9741$ & $\overline{1} .9743 \bar{I}$ & $\bar{x}$ & (1) & $.9751 \bar{I}$ & 54 & 19 & 321 \\
\hline 71 & 9757 & 9759 & 9762 & 9764 & 9767 & 9770 & $977^{2}$ & 9775 & 9777 & 9780 & is & 000 \\
\hline $7^{2}$ & 9782 & 9785 & 9787 & 9789 & 9792 & 9794 & 9797 & 9799 & 9801 & 9804 & 17 & 100 \\
\hline 73 & 9806 & 9808 & 9811 & 9813 & 9815 & 9817 & 9820 & $9 \$ 22$ & 9824 & $9 \$ 26$ & 16 & 110 \\
\hline 74 & $\begin{array}{r}9828 \\
-\quad 8\end{array}$ & 9831 & 9833 & $9^{8} 35$ & 9837 & 9839 & $984 r^{\circ}$ & 9843 & 9845 & 9847 & 15 & 110 \\
\hline 75 & '..9849 & i. 98517 & 1.9853 & i. 9855 & I. .9857 & '. 9859 i & i. $9861 \overline{1}$ & $.980_{3} \mathrm{I}$ & $.9805 \overline{1}$ & $.9 \$ 67$ & 14 & 211 \\
\hline 76 & $98(x)$ & $9^{8} 71$ & $9^{873}$ & 9875 & 9876 & $9^{8} 7^{8}$ & 9880 & $98 S_{2}$ & 9884 & $98 s_{5}$ & 13 & 211 \\
\hline 77 & 9887 & 9889 & 9891 & 9892 & 9894 & $98 x, 6$ & 9897 & 9899 & 9901 & 9002 & 12 & 211 \\
\hline $7^{8}$ & 9904 & 9906 & 9907 & 9909 & 9910 & 9912 & 9913 & 9915 & 9916 & 9918 & 11 & 221 \\
\hline 79 & 9919 & 9921 & 9922 & 9924 & 9925 & 9927 & 9928 & 9929 & 9931 & 9932 & 10 & 321 \\
\hline $80^{\circ}$ & $\bar{I} .9034 \mathrm{I}$ & i.9935 i & T.9936 & T.9937 & T. 9939 & I. 9940 I & I. 9941 i & (.9943 & 99441 & .9945 & 9 & 21 \\
\hline $8 i$ & $994^{6}$ & 9947 & 9949 & 9950 & 9951 & 9952 & 9953 & 9954 & 9955 & $995^{6}$ & 8 & 00 \\
\hline 82 & $995^{8}$ & 9959 & 9960 & $996 \mathrm{x}$ & 9962 & 9063 & 9964 & 9065 & 9966 & 9967 & 7 & 00 \\
\hline 83 & 9968 & 9968 & $99(x)$ & 9970 & 9971 & 9972 & 9973 & 9974 & 99 & 9975 & 6 & 10 \\
\hline 84 & $997^{6}$ & 9977 & $997^{8}$ & $997^{8}$ & 9979 & 99 so & 9088 & gosi & 9982 & $x_{0} 8_{3}$ & 5 & 10 \\
\hline 85 & T. $998_{3} \overline{\mathrm{I}}$ & $.9984 i$ & .9985 i & .9985 i & I. $.99 \$ 6$ & ז. $90 \delta_{7}$ ז & .9987 i & .99851 & $.9985 \mathrm{~T}$ & .9089 & 4 & 11 \\
\hline 86 & 99.89 & 9990 & 9900 & 9991 & 9991 & 9992 & 9992 & 9993 & 9993 & 9994 & 3 & 11 \\
\hline 87 & 9904 & y994 & 9005 & 9905 & 9096 & 9906 & 9996 & 9096 & 9997 & 9997 & 2 & 11 \\
\hline 88 & 9007 & 9998 & 9008 & 9098 & 9008 & $90 \times(9)$ & $9 x 9$ & 9909 & 9909 & 9000 & 1 & 21 \\
\hline \multirow[t]{2}{*}{80} & 9699 & 9090 & zero & zero & zero & zero & zero & zero & zeri) & nere & 0 & 21 \\
\hline & 1.0 & & & .8 & & & & & 8 & ${ }^{\circ}, \mathrm{I}$ & Deg. & $\begin{array}{l}\text { Interpole. } \\
\text { for h dthe }\end{array}$ \\
\hline
\end{tabular}




\section{LOGARITHMS OF TANGENTS AND COTANGENTS}

TO

FOUR PLACES.

Note. For log. cot. use right-hand column of degrees and lower line of tenth

\begin{tabular}{|c|c|c|c|c|c|c|c|c|c|c|c|c|c|}
\hline Deg. & $\because 0$ & $\stackrel{\circ}{\circ}$ & $\stackrel{\circ}{2}$ & $\cdot 3$ & $\stackrel{\circ}{\circ} 4$ & ${ }^{\circ} \cdot 5$ & .6 & $\cdot 7$ & $\circ .8$ & .9 & & \multicolumn{2}{|c|}{$\begin{array}{l}\text { In:erpola } \\
\text { for } h \text { dthe }\end{array}$} \\
\hline $0^{\circ}$ & \multicolumn{5}{|c|}{$-\infty \overline{3} .2419 \overline{\overline{3}} .5429 \overline{\overline{3}} \cdot 7190 \overline{\underline{3}} .8439$} & \multicolumn{5}{|c|}{$\overline{\mathbf{3}} .9409 \overline{\mathbf{2}} .0200 \overline{2} .0870 \overline{\mathbf{2}} .1450 \overline{\mathbf{2}} .1962$} & 89 & & \\
\hline I & \multirow{2}{*}{\multicolumn{5}{|c|}{$\overline{2} .5431 \overline{2} .5643 \overline{2} .5845 \overline{2} .6038 \overline{2} .6223$}} & \multirow{2}{*}{\multicolumn{5}{|c|}{ 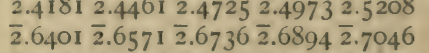 }} & 88 & 4 & 3 \\
\hline 2 & & & & & & & & & & & 87 & 7 & 5 \\
\hline 3 & \multirow{2}{*}{\multicolumn{5}{|c|}{ 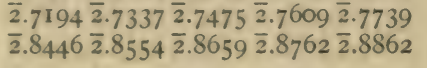 }} & \multicolumn{5}{|c|}{$\overline{2} .7865 \overline{2} .7988 \overline{2} .8107 \overline{2} .8223 \overline{2} .8336$} & 86 & II & \\
\hline 4 & & & & & & & $=700-7$ & $\overline{2} .9150 \overline{2}$ & $2.9241 \overline{2}$ & $\overline{2} .9331$ & 85 & 14 & II \\
\hline 5 & \multirow{2}{*}{\multicolumn{5}{|c|}{ 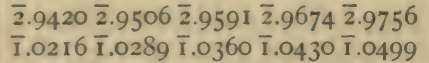 }} & \multicolumn{5}{|c|}{$\overline{2} .9836 \overline{2} .9915 \overline{2} .9992 \overline{\mathbf{1}} .0068 \overline{\mathbf{1}} .0143$} & 84 & 18 & 14 \\
\hline 6 & & & & & & $\overline{1} .0567$ & {$[.0633]$} & $\overline{\mathrm{I}} .0699 \overline{\mathrm{I}}$ & $\bar{I} .0764 \bar{I}$ & I. .0828 & 83 & $2 \mathrm{I}$ & 16 \\
\hline 7 & \multicolumn{2}{|c|}{$\overline{\mathrm{I}} .089 \mathrm{I} \overline{\mathrm{I}} .0954$} & $\overline{\mathrm{I}} . \mathrm{IOI}_{5} \overline{\mathrm{I}}$ & I.I & $\overline{\mathbf{I}} . \mathrm{I}$ I 35 & \multicolumn{5}{|c|}{ 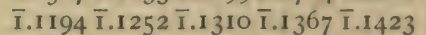 } & 82 & 25 & 19 \\
\hline 8 & \multirow{2}{*}{\multicolumn{2}{|c|}{$\begin{array}{l}\overline{\mathrm{I}} .1478 \overline{\mathrm{I}} .1533 \\
\overline{\mathrm{I}} .1997 \overline{\mathrm{I}} .2046\end{array}$}} & $\overline{\mathrm{I}} .15$ & 1 & I.1693 & \multirow{2}{*}{\multicolumn{5}{|c|}{ 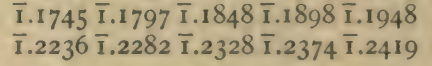 }} & $8 \mathrm{I}$ & 28 & 22 \\
\hline 9 & & & $\overline{\mathbf{I}}$. & & 189 & & & & & & $80^{\circ}$ & 32 & 24 \\
\hline $10^{\circ}$ & \multicolumn{5}{|c|}{ 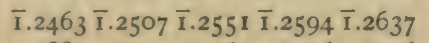 } & \multicolumn{5}{|c|}{$\overline{\mathrm{I}} .2680 \overline{\mathrm{I}} .2722 \overline{\mathrm{I}} .2764 \overline{\mathrm{I}} .2805 \overline{\mathrm{I}} .2846$} & 79 & 25 & 23 \\
\hline II & 2887 & 2927 & 2967 & 3006 & 3046 & 3085 & 3123 & 3162 & 3200 & 3237 & 78 & 3 & 2 \\
\hline 12 & 3275 & $33^{12}$ & 3349 & $33^{8} 5$ & 3422 & $345^{8}$ & 3493 & 3529 & 3564 & 3599 & 77 & 5 & 5 \\
\hline 13 & 3634 & 3668 & 3702 & $373^{6}$ & 3770 & 3804 & $3^{8} 37$ & $3^{8} 70$ & 3903 & 3935 & 76 & 8 & 7 \\
\hline 14 & 3968 & 4000 & 4032 & 4064 & 4095 & 4127 & $4^{1} 5^{8}$ & 4189 & 4220 & 4250 & 75 & 10 & 9 \\
\hline 15 & \multicolumn{5}{|c|}{$\overline{\mathrm{I}} .428$ I I. .43 II $\overline{\mathrm{I}} .434$ I $\overline{\mathrm{I}} .437$ I I .4400} & \multicolumn{5}{|c|}{$\overline{\mathrm{I}} \cdot 4430 \overline{\mathrm{I}} \cdot 4459 \overline{\mathrm{I}} \cdot 4488 \overline{\mathrm{I}} \cdot 45 \mathrm{I} 7 \overline{\mathrm{I}} \cdot 4546$} & 74 & 13 & 12 \\
\hline 16 & 4575 & 4603 & 4632 & 4660 & 4688 & 4716 & 4744 & 4771 & 4799 & 4826 & 73 & 15 & 14 \\
\hline 17 & 4853 & 4880 & 4907 & 4934 & 4961 & 4987 & 5014 & 5040 & 5066 & 5092 & 72 & 18 & 16 \\
\hline 18 & 5118 & $5^{143}$ & $5^{169}$ & 5195 & 5220 & 5245 & 5270 & 5295 & 5320 & 5345 & 71 & 20 & 18 \\
\hline 19 & 5370 & 5394 & 5419 & 5443 & 5467 & 5491 & 5516 & 5539 & $55^{6} 3$ & $55^{87}$ & $70^{\circ}$ & 23 & $2 I$ \\
\hline $20^{\circ}$ & \multicolumn{5}{|c|}{$\overline{\mathrm{I}} .561 \mathrm{I} \overline{\mathrm{I}} .5634 \overline{\mathrm{I}} .565^{8} \overline{\mathrm{I}} .568 \mathrm{I} \overline{\mathrm{I}} \cdot 5704$} & \multicolumn{5}{|c|}{$\overline{\mathrm{I}} .5727 \overline{\mathrm{I}} .5750 \overline{\mathrm{I}} .5773 \overline{\mathrm{I}} .5796 \overline{\mathrm{I}} .5819$} & 9 & 21 & 19 \\
\hline 21 & 5842 & 5864 & 5887 & 5909 & 5932 & 5954 & 5976 & 5998 & 6020 & 6042 & 8 & 2 & 2 \\
\hline 22 & 6064 & 6086 & 61 & 61 & $6 I$ & & 61 & 5 & 6 & 6257 & 67 & 4 & 4 \\
\hline 23 & 6279 & 6300 & 6321 & 6341 & 6362 & 6383 & 6404 & 6424 & 6445 & 6465 & 66 & 6 & 6 \\
\hline 24 & 6486 & 6506 & 6527 & 6547 & 6567 & 6587 & 6607 & 6627 & 6647 & 6667 & 65 & 8 & 8 \\
\hline 25 & \multicolumn{5}{|c|}{$\overline{\mathrm{I}} .6687 \overline{\mathrm{r}} .6706 \overline{\mathrm{r}} .6726 \overline{\mathrm{I}} .6746 \overline{\mathrm{r}} .6765$} & \multicolumn{5}{|c|}{ 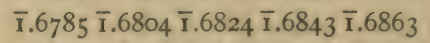 } & 64 & II & 10 \\
\hline 26 & 6882 & 6901 & 6920 & 6939 & 6958 & 6977 & 6996 & 7015 & 7034 & 7053 & 63 & 13 & II \\
\hline 27 & 7072 & 7090 & 7109 & 7128 & 7146 & 7165 & 7183 & 7202 & 7220 & 7238 & 62 & 15 & 13 \\
\hline 28 & 7257 & 7275 & 7293 & 7311 & 7330 & 7348 & 7366 & 7384 & 7402 & 7420 & $6 \mathbf{r}$ & 17 & 15 \\
\hline 29 & $743^{8}$ & 7455 & 7473 & $749 \mathrm{I}$ & 7509 & 7526 & 7544 & $75^{62}$ & 7579 & 7597 & $60^{\circ}$ & 19 & 17 \\
\hline $30^{\circ}$ & $\overline{\mathrm{I}} \cdot 7614$ & $\overline{\mathrm{I}} .7632$ & $\overline{\mathrm{I}} .7649 \overline{\mathrm{I}}$ & .7667 & $\overline{\mathbf{I}} \cdot 7684$ & $\overline{\mathbf{I}} \cdot 77$ & $\overline{1} .7719$ & $6 \overline{\mathrm{I}}$ & $\overline{\mathbf{I}}$ & $\mathbf{1} \cdot 777^{1}$ & 59 & 17 & 15 \\
\hline $3 I$ & 7788 & 7805 & 7822 & 7839 & 7856 & 7873 & 7890 & 7907 & 7924 & 7941 & $5^{8}$ & 2 & 2 \\
\hline 32 & $795^{8}$ & 7975 & 7992 & 8008 & 8025 & $80_{42}$ & $80_{59}$ & 8075 & 8092 & 8109 & 57 & 3 & 3 \\
\hline 33 & 8125 & $8 \mathbf{I}_{4} 2$ & $815^{8}$ & 8175 & 8191 & 8208 & 8224 & 8241 & 8257 & 8274 & 56 & 5 & 5 \\
\hline 34 & 8290 & 8306 & 8323 & 8339 & 8355 & 8371 & 8388 & 8404 & 8420 & 8436 & 55 & 7 & 6 \\
\hline 35 & $\overline{1} .845^{2}$ & I. 8468 & $\overline{\mathrm{I}} .8484 \overline{\mathrm{I}}$ & .8501 & $\overline{\mathbf{I}} .8517$ & $\overline{\mathbf{1}} .8533$ & I. 8549 & $\overline{\mathrm{I}} .8565 \overline{\mathrm{I}}$ & $\overline{\mathrm{I}} .858 \mathrm{I} \overline{\mathrm{I}}$ & $\overline{\mathbf{x}} .8597$ & 54 & 9 & 8 \\
\hline 36 & 8613 & 8629 & 8644 & 8660 & 8676 & 8692 & 8708 & 8724 & 8740 & 8755 & 53 & 10 & 9 \\
\hline 37 & 8771 & 8787 & $880_{3}$ & 8818 & 8834 & 8850 & 8865 & 888 I & 8897 & 8912 & $5^{2}$ & 12 & II \\
\hline 38 & 8928 & 8944 & 8959 & 8975 & 8990 & 9006 & 9022 & 9037 & 9053 & 9068 & 51 & 14 & 12 \\
\hline 39 & 9084 & 9099 & 9115 & 9130 & 9146 & $916 I$ & 9176 & 9192 & 9207 & 9223 & $50^{\circ}$ & 15 & I4 \\
\hline & $\mathbf{r}^{\circ}$. & & & & & & & & \% & I & Deg. & & \\
\hline
\end{tabular}


4 PL. LOG. TAN.

\begin{tabular}{|c|c|c|c|c|c|c|c|c|c|c|c|c|}
\hline Deg. & $\circ 0$ & . $I$ & .2 & $\cdot 3$ & $\cdot 4$ & ${ }^{\circ} \cdot 5$ & .6 & ${ }^{\circ} .7$ & .8 & .9 & & $\begin{array}{l}\text { Interpola. } \\
\text { for hidths }\end{array}$ \\
\hline $40^{\circ}$ & …9238 & .92541 & $9269 \overline{1}$ & $.92 S_{4} \mathrm{I}$ & 1.9300 & $\overline{\mathrm{I}} .9315 \overline{\mathrm{I}}$ & $.9330 \mathrm{I}$ & $8.9346 \bar{i}$ & $.9361 \overline{1}$ & $\overline{\mathbf{I}} .9376$ & 49 & 1516 \\
\hline 41 & I. 9392 & $.9407 \mathrm{I}$ & $.9422 \mathrm{I}$ & $.9438 \mathrm{I}$ & 1. $9+53$ & I. $9468 \overline{\mathrm{I}}$ & $.94 S_{3} \mathrm{I}$ & $1.9499 \mathrm{I}$ & $.95^{1}+1$ & 1. 9529 & 48 & 22 \\
\hline 42 & I. 9544 & $.9560 \mathrm{I}$ & $.9575^{\mathrm{I}}$ & .95901 & 1.9605 & J. 9621 I & $.9636 \mathrm{I}$ & $1.9651 \mathrm{I}$ & $7.9666 \mathrm{I}$ & I. $968 \mathrm{I}$ & 47 & 33 \\
\hline 43 & i. 9697 & .97121 & $1.9727 \mathrm{I}$ & $.9742 \mathrm{I}$ & 1.9757 & I. 9772 i & $.9788 \mathrm{I}$ & ${ }^{7} .9 \mathrm{SO}_{3} \mathrm{I}$ & I. $9 S_{1} \mathrm{~S}_{\mathrm{I}}$ & 1. 9833 & 46 & 55 \\
\hline 44 & T. 9848 & $.9 \mathrm{SG}_{4} \mathrm{I}$ & $1.9879 \mathrm{I}$ & $.9894 \overline{1}$ & 1.9909 & I. 9924 I & I.9939 & I. $9955 \mathrm{I}$ & 1.99701 & 1. .9985 & 45 & 66 \\
\hline 45 & 0.0000 & $.0015 \mathrm{c}$ & $.0030 \mathrm{C}$ & .00450 & 0.0061 & 0.00760 & $.0091 \mathrm{c}$ & 0.01060 & $0.0121 \mathrm{C}$ & 0.0136 & 44 & 88 \\
\hline 46 & 0152 & 0167 & 0182 & 0197 & 0212 & 0228 & 0243 & $025^{8}$ & 0273 & 0288 & 43 & 910 \\
\hline 47 & 0303 & 0319 & 0334 & 0349 & 0364 & 0379 & 0395 & 0410 & 0425 & 0440 & 42 & II II \\
\hline 48 & 0456 & 0471 & 0.486 & 0501 & 0517 & 0532 & 0547 & 0562 & $057^{8}$ & 0593 & $4 I$ & 1213 \\
\hline 49 & 0608 & 0624 & 0639 & 0654 & 0670 & $068_{5}$ & 0700 & 0716 & 0731 & 0746 & $40^{\circ}$ & 1414 \\
\hline $50^{\circ}$ & 0.0762 & $.0777 \mathrm{C}$ & 0.07930 & .08080 & 0.0824 & 0.08390 & $.0854 \mathrm{C}$ & $0.0870 \mathrm{c}$ & $.0885 \mathrm{c}$ & 0.0901 & 39 & 1718 \\
\hline $5^{1}$ & 0916 & 0932 & 0947 & 0963 & 0978 & 0994 & 1010 & 1025 & 1041 & 1056 & 38 & 22 \\
\hline $5^{2}$ & 1072 & 1088 & 1103 & 1119 & 1135 & 1150 & 1166 & 1182 & 1197 & 1213 & 37 & 34 \\
\hline 53 & 1229 & 1245 & 1260 & 1276 & 1292 & 1308 & 1324 & 1340 & 1356 & 1371 & 36 & 55 \\
\hline 54 & ${ }_{13} \delta_{7}$ & 1403 & 1419 & 1435 & 1451 & 1467 & 1483 & 1499 & 1516 & 1532 & 35 & 77 \\
\hline 55 & $0.154^{8}$ & $.1564 c$ & $.15^{80} \mathrm{c}$ & .15960 & 0.1612 & 0.16290 & $0.1645 c$ & $0.1661 \mathrm{c}$ & $0.1677 c$ & 0.1694 & 34 & 99 \\
\hline 56 & 1710 & 1726 & 1743 & 1759 & 1776 & 1792 & 1809 & 1825 & 1842 & $185^{8}$ & 33 & $10 \mathrm{II}$ \\
\hline 57 & 1875 & 1891 & 1908 & 1925 & 1941 & $195^{8}$ & 1975 & 1992 & 2008 & 2025 & 32 & 1213 \\
\hline $5^{8}$ & 2042 & 2059 & 2076 & 2093 & 2110 & 2127 & 2144 & $216 I$ & 2178 & 2195 & 31 & I 4.14 \\
\hline 59 & 2212 & 2229 & 2247 & 2264 & $228 I$ & 2299 & 2316 & 2333 & 2351 & 2368 & $30^{\circ}$ & 1516 \\
\hline $60^{\circ}$ & 0.2386 & $.2403 c$ & $0.2421 \mathrm{C}$ & 0.243 & 0.2456 & 0 & $0.249 \mathrm{IC}$ & 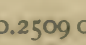 & $0.2527 \mathrm{C}$ & 0.2545 & 29 & 2123 \\
\hline 61 & 2562 & 2580 & 2598 & 2616 & 2634 & 2652 & 2670 & 2689 & 2707 & 2725 & 28 & 22 \\
\hline 62 & 2743 & 2762 & 2780 & 2798 & 2817 & 2835 & 2854 & 2872 & $289 i$ & 2910 & 27 & 45 \\
\hline 63 & 2928 & 2947 & 2966 & 2985 & 3004 & 3023 & 3042 & 3061 & 3080 & 3099 & 26 & 67 \\
\hline 64 & 3118 & 3137 & 3157 & 3176 & 3196 & 3215 & 3235 & 3254 & 3274 & 3294 & 25 & 89 \\
\hline 65 & 0.3313 & .33330 & $.3353 c$ & .33730 & 0.3393 & 0.34130 & $.3433 c$ & 0.34530 & $0.3473 c$ & 0.3494 & 24 & II 12 \\
\hline 66 & 3514 & 3535 & 3555 & $357^{6}$ & 3596 & 3617 & 3638 & 3659 & 3679 & 3700 & 23 & 1314 \\
\hline 67 & 3721 & 3743 & 3764 & $37^{8} 5$ & 3806 & 3828 & 3849 & $3^{871}$ & 3892 & 3914 & 22 & 1516 \\
\hline 68 & 3936 & $395^{8}$ & 3980 & 4002 & 4024 & 4046 & 4068 & 409 I & 4113 & 4136 & 21 & 1718 \\
\hline 69 & $4^{1} 5^{8}$ & 4181 & 4204 & 4227 & 4250 & 4273 & 4296 & 4319 & $434^{2}$ & 4366 & $20^{\circ}$ & 1921 \\
\hline $70^{\circ}$ & 0.4389 & .44130 & $.4437 \mathrm{C}$ & $.4461 \mathrm{c}$ & 0.4484 & 0.45090 & $0.4533 c$ & 0.45570 & $0.45^{81} \mathrm{C}$ & 0.4606 & 19 & 2527 \\
\hline 71 & 4630 & 4655 & 4680 & 4705 & 4730 & 4755 & 4780 & $4 \mathrm{SO}_{5}$ & $4 S_{31}$ & $4 S_{57}$ & 18 & 33 \\
\hline 72 & 4882 & 4908 & 4934 & 4960 & 4986 & 5013 & 5039 & 5066 & 5093 & 5120 & 17 & 55 \\
\hline 73 & $5^{1} 47$ & 5174 & 5201 & 5229 & 5256 & 5284 & 5312 & 5340 & 5368 & 5397 & 16 & 88 \\
\hline 74 & 5425 & 5454 & 5483 & 5512 & 5541 & 5570 & 5600 & 5629 & 5659 & 5689 & 15 & Io II \\
\hline 75 & 0.5719 & 575 & $5 ;-500$ & .58110 & $0.5^{8} 4^{2}$ & $0.5^{8730}$ & $.5905 \mathrm{C}$ & .59360 & $0.5968 c$ & 0.6000 & 14 & 1314 \\
\hline 76 & 6032 & 6065 & 6097 & 6130 & 6163 & 6196 & 6230 & 6264 & 6298 & 63,32 & 13 & 1516 \\
\hline 77 & 6366 & 6401 & 6436 & 6471 & 6507 & 6542 & $657^{8}$ & 6615 & 6651 & 6658 & 12 & 1819 \\
\hline 78 & 6725 & 6763 & 6800 & 6838 & 6877 & 6915 & 6954 & 6094 & 7033 & 7073 & II & 2022 \\
\hline 79 & 7113 & 7154 & 7195 & 7236 & 7278 & 7320 & 7363 & 7406 & 7449 & 7493 & $10^{\circ}$ & 2324 \\
\hline $80^{\circ}$ & 0.7537 & .75810 & 2.7626 & .767 & 0.7718 & 0.77640 & $0.7811 \mathrm{C}$ & .78580 & $0.7906 \mathrm{c}$ & rer & 9 & 8546 \\
\hline 81 & $0.8 \mathrm{OOO}_{3}$ &. $\mathrm{SO}_{52} 20$ & $.8102 \mathrm{C}$ & .81520 & 0.8203 & 0.82550 & $.8307 \mathrm{C}$ & $0.8,3600$ & $0 . S_{4} 13 \mathrm{C}$ & 0.8467 & 8 & 45 \\
\hline 82 & 0.8522 & 85770 & .86330 & $.86 \operatorname{loc}^{\circ}$ & 0.8748 & 0.55060 & $0.8865 \mathrm{C}$ & $0.8 y 240$ & $0 . \mathrm{SgS}_{5} \mathrm{C}$ & 0.9046 & 7 & 79 \\
\hline $8_{3}$ & 0.9109 & .01720 & .92360 & .93010 & 0.9367 & 0.94330 & $0.9501 \mathrm{C}$ & 0.95700 & $0.9640 \mathrm{c}$ & 0.9711 & 6 & 1114 \\
\hline 84 & 0.9784 & .98570 & .9932 & 1.0008 & 1.0085 & 1.01641 & 1.0244 & 1.03261 & 1.0409 & 1.0494 & 5 & 1418 \\
\hline 85 & 1.0580 & .086091 & 1.0759 & 1.08501 & 1.0944 & 1.10401 & 1.1138 & 1.12381 & $1.134 t$ & 1.14 & 4 & $18 \quad 23$ \\
\hline 86 & 1.1554 & $.1664:$ & 1.17771 & 1.18031 & 1.2012 & 1.21351 & 1.2261 & 1.23911 & 1.2525 & 1.2663 & 3 & 2127 \\
\hline 87 & 1.2506 & $.2054 \mathrm{I}$ & 1.31001 & 1.32641 & 1.3429 & 1. $3599 \mathrm{I}$ & .3777 & 1.3962 I & 1.4155 & 1.4357 & 2 & 2532 \\
\hline 88 & $1.45(x)$ & 47921 & 1.5027 & 1.5275 & 1.5539 & $1.5 \sin 1$ & 1.6110 & 1.64 .418 & 1. $6,-50$ & 1.7167 & 1 & 2836 \\
\hline \multirow[t]{2}{*}{89} & 1.7581 & $\operatorname{son}_{3} S_{1}$ & .8550 & .91301 & 1.9500 & 2.05912 & $2.1561=$ & $2.2810^{2}$ & 2.4571 & $2.75^{8 i}$ & $0^{\circ}$ & 3241 \\
\hline & & & 0. & & & & & & 2 & $\because .1$ & Dog. & $\begin{array}{l}\text { Interpola. } \\
\text { for th dths }\end{array}$ \\
\hline
\end{tabular}





\section{LOGARITHMS OF TRIGONOMETRIC FUNCTIONS}

TO

\section{FIVE PLACES.}

Note. The table gives the log of the natural value of the function, and hence the characteristic is negative when that value is fractional. The common practice of adding 10. to avoid the negative characteristic is not recommended.

\begin{tabular}{|c|c|c|c|c|c|}
\hline $0^{\circ}$ & $\log \cos$ & & $0^{\circ}$ & $\log 008$ & \\
\hline $\begin{array}{r}0^{\prime}-16^{\prime} \\
17^{\prime}-28^{\prime} \\
29^{\prime}-36^{\prime} \\
37^{\prime}-43^{\prime}\end{array}$ & $\begin{array}{l}0.00000 \\
\overline{\mathbf{I}} .99999 \\
\overline{\mathbf{I}} .99998 \\
\overline{\mathbf{x}} .99997\end{array}$ & $\begin{array}{l}44^{\prime}-60^{\prime} \\
32^{\prime}-43^{\prime} \\
24^{\prime}-31^{\prime} \\
17^{\prime}-23^{\prime}\end{array}$ & $\begin{array}{r}44^{\prime}-49^{\prime} \\
50^{\prime}-54^{\prime} \\
55^{\prime}-59^{\prime} \\
60^{\prime}\end{array}$ & $\begin{array}{l}\overline{1} .99996 \\
\overline{\mathbf{1}} .99995 \\
\overline{\mathbf{x}} .99994 \\
\overline{\mathbf{x}} .99993\end{array}$ & $\begin{array}{l}11^{\prime}-16^{\prime} \\
6^{\prime}-10^{\prime} \\
I^{\prime}-5^{\prime} \\
d^{\prime}\end{array}$ \\
\hline & $\log \sin$ & $89^{\circ}$ & & $\log$ in & $89^{\circ}$ \\
\hline
\end{tabular}

$0^{\circ} \quad 0^{\circ}$

\begin{tabular}{|c|c|c|c|c|c|c|c|c|c|}
\hline 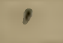 & $\log \sin$ & $\log \tan$ & $\log$ oot & & ' & $\log \sin$ & $\log \tan$ & $\log$ oot & \\
\hline $0^{\prime}$ & $\bar{\infty}$ & $\bar{\infty}$ & $\infty$ & $60^{\prime}$ & $30^{\prime}$ & $\overline{3} .94084$ & $\overline{3} .94086$ & 2.05914 & $30^{\prime}$ \\
\hline i & $\overline{4} \cdot 4^{6} 373$ & $\overline{4} \cdot 46373$ & 3.53627 & 59 & $3 i$ & 95508 & 95510 & 04490 & 29 \\
\hline 2 & $47^{6}+7^{0}$ & $4.7647^{6}$ & 3.23524 & $5^{8}$ & 32 & 96887 & 96889 & 03 III & $2 \hat{8}$ \\
\hline 3 & $4 y+0_{5}$ & 4.94085 & 3.05915 & 57 & 33 & 98223 & $9^{8} 225$ & ol 775 & 27 \\
\hline 4 & $\overline{3} 06579$ & $\overline{3} 06579$ & 2.93421 & 56 & 34 & $\overline{3} .99520$ & $\overline{3} .99522$ & $2.00+78$ & 26 \\
\hline 5 & $\overline{3} .16270$ & $\overline{3} .16270$ & 2.83730 & 55 & 35 & $\overline{2} .00779$ & $\overline{2} .0078 \mathbf{I}$ & 1.99219 & 25 \\
\hline 6 & 24158 & 24188 & 75812 & 54 & 36 & 02002 & 02004 & 97996 & 24 \\
\hline 7 & $30 \mathrm{SS}_{2}$ & $30 \$ 22$ & 69118 & 53 & 37 & 03192 & 03194 & 96806 & 23 \\
\hline 8 & $3^{(i) 6 S 2}$ & 36682 & 63318 & $5^{2}$ & $3^{8}$ & 04350 & 04353 & 95647 & 22 \\
\hline 9 & 41797 & 41797 & $5^{8203}$ & 51 & 39 & $0547^{8}$ & $0548 i$ & 94519 & 21 \\
\hline 10 & $\overline{3} .46 .373$ & $\overline{3} \cdot 4^{6} 373$ & 2.53627 & 50 & 40 & $\overline{2} .0657^{8}$ & $\overline{2} .0658 \mathrm{i}$ & 1.93419 & 20 \\
\hline 11 & 50512 & 50512 & 49488 & 49 & 41 & 07650 & 07053 & 92.347 & 19 \\
\hline 12 & $5429 \mathrm{t}$ & 54201 & 45709 & $4^{8}$ & 42 & $0 \$(x) 6$ & 05700 & 91 300 & is \\
\hline 13 & 57767 & $577^{67}$ & 42233 & 47 & 43 & a) 718 & 09722 & 90278 & 17 \\
\hline 14 & 6 ys 5 & 60956 & 39014 & 46 & 44 & 10717 & 10720 & 89280 & 16 \\
\hline 15 & $\overline{3} .639 \$ 2$ & $\overline{3} .63982$ & 2.36018 & 45 & 45 & $\overline{2.11693}$ & 2.11696 & $1.85,304$ & 15 \\
\hline 16 & 66784 & $66-85$ & 33215 & 44 & 46 & 12647 & 12651 & 87340 & 14 \\
\hline 17 & $6 \times 9417$ & $69+18$ & 30582 & 43 & 47 & $135^{8 i}$ & $135^{x_{5}}$ & 86415 & 13 \\
\hline 18 & 71900 & 71900 & 28100 & 42 & $4^{8}$ & 14495 & 14500 & $\$ 5500$ & 12 \\
\hline 19 & $7424^{8}$ & 74248 & 25752 & $4^{1}$ & 49 & 15391 & 15.395 & 84605 & 11 \\
\hline 20 & $\overline{3} \cdot 76475$ & $\overline{3} .76476$ & 2.23524 & 40 & 50 & $\overline{2} .1626 .8$ & $\overline{2} .1627 .3$ & 1. $\$ 3727$ & 10 \\
\hline 21 & 75594 & 785175 & 21405 & 3) & 51 & 17128 & 17133 & $\mathrm{~S}_{2} \mathrm{Si}_{7}$ & 9 \\
\hline 22 & Sohis 5 & 80615 & 1935 & 38 & $5^{2}$ & 17971 & 17076 & 82024 & 8 \\
\hline 23 & 82545 & $8254^{6}$ & $\begin{array}{l}17454 \\
15606\end{array}$ & 37 & 53 & $\begin{array}{l}18795 \\
10610\end{array}$ & $\begin{array}{l}18504 \\
10616\end{array}$ & $\begin{array}{l}\text { S1 } 196 \\
\text { So } 354\end{array}$ & $\frac{7}{6}$ \\
\hline 24 & 84,373 & 84394 & 15606 & $3^{6}$ & 54 & 19610 & 19616 & $303^{3} 4$ & 0 \\
\hline 25 & 3.861660 & 3. 96167 & 2.13833 & 35 & 55 & $\overline{2} .20407$ & $\begin{array}{r}2.20+113 \\
21105\end{array}$ & 1.79587 & 5 \\
\hline $\begin{array}{l}20 \\
27\end{array}$ & $\begin{array}{l}57570 \\
8050(x)\end{array}$ & $8757 t$ & 12129 & 34 & 56 & 21159 & $\begin{array}{ll}21 & 105 \\
21 & 964\end{array}$ & 78036 & 4 \\
\hline 28 & 91 uss & 91 using & os 911 & $\begin{array}{l}33 \\
32\end{array}$ & $\begin{array}{l}57 \\
58\end{array}$ & 22713 & 22720 & 77250 & 2 \\
\hline 29 & 92612 & 92613 & 07357 & 31 & 59 & $2345^{6}$ & 23462 & $7653^{8}$ & 1 \\
\hline \multirow[t]{2}{*}{$30^{\prime}$} & 3.94084 & 3.94086 & 2.05914 & $30^{\prime}$ & $60^{\prime}$ & $\overline{2} .24186$ & $\overline{2} .24192$ & 1.75808 & $0^{\prime}$ \\
\hline & $\log \cos$ & $\log$ oot & $\log \tan$ & , & & $\log 008$ & $\log \cot$ & $\log \tan$ & 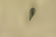 \\
\hline
\end{tabular}




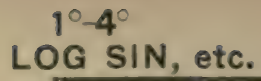

$1^{\circ}$

2

\begin{tabular}{|c|c|c|c|c|c|c|c|c|c|}
\hline I & $\log \sin$ & $\log \tan$ & $\log \cot$ & $\log \cos$ & $\log \sin$ & $\log \tan$ & $\log \cot$ & $\log c 08$ & \\
\hline $0^{\prime}$ & $\overline{2} .24186$ & $\overline{2} .24192$ & $1.75 \mathrm{SOS}$ & I. 99993 & $\overline{2} .54282$ & $\overline{2} \cdot 54308$ & 1.45692 & $\overline{1} .99974$ & $60^{\prime}$ \\
\hline 1 & 24903 & 24910 & $75 \circ 90$ & 99993 & 54642 & 54669 & 45331 & 99973 & 59 \\
\hline 2 & 25609 & 25616 & $7+354$ & 99993 & 54999 & 55027 & 44973 & 99973 & $5^{5}$ \\
\hline 3 & 26304 & 26312 & 73688 & 99993 & 55354 & 55382 & 44618 & 99972 & 57 \\
\hline 4 & 26985 & 26996 & 73004 & 99992 & 55705 & 55734 & 44266 & 99972 & 56 \\
\hline 5 & $\overline{2} .2766 \mathrm{I}$ & $\overline{2} .27669$ & 1.72331 & '..99992 & $\overline{2} .56054$ & $\overline{2} .56083$ & 1.43917 & $\overline{\mathbf{I}} .99971$ & 55 \\
\hline 6 & 28324 & 28332 & 71608 & 99992 & 56400 & 56429 & 43571 & 99971 & 54 \\
\hline 7 & 28977 & 28956 & 71014 & 99992 & $5^{6} 743$ & $5^{6} 773$ & 43227 & 99970 & 53 \\
\hline 8 & 29621 & 29629 & $7037 \mathrm{I}$ & 99992 & 57084 & $57 \because 14$ & 42886 & 99970 & $5^{2}$ \\
\hline 9 & 30255 & 30263 & 69737 & 99991 & 57421 & $5745^{2}$ & $4^{2} 54^{8}$ & 99969 & 51 \\
\hline 10 & $\overline{\mathbf{2}} \cdot 30879$ & $\overline{2} .30888$ & 1.69112 & $\overline{\text { I. }} 9999$ I & $\overline{2} .57757$ & $\overline{2} .57788$ & I. $422 \mathrm{I} 2$ & $\overline{\text { I. }} 99969$ & 50 \\
\hline II & 31495 & 31505 & 68495 & 99991 & 58089 & $5^{8121}$ & 41879 & 99968 & 49 \\
\hline 12 & 32103 & 32 I1 & 67858 & 99990 & $5^{8} 419$ & $5^{8} 45^{1}$ & 41549 & 99968 & 48 \\
\hline 13 & 32702 & 32711 & 67289 & 99990 & $5^{8} 747$ & $5^{8} 779$ & $4122 I$ & 99967 & 47 \\
\hline 14 & 33292 & 33302 & 66698 & 99990 & 59072 & 59105 & 40895 & 99967 & 46 \\
\hline 15 & $\overline{2} .33875$ & $\overline{2} .33886$ & I. 66 I I 4 & $\overline{\mathbf{I}} .99990$ & $\overline{2} .59395$ & $\overline{2} .59428$ & I. $4057^{2}$ & $\overline{\mathbf{1}} .99967$ & 45 \\
\hline 16 & $3445^{\circ}$ & 34461 & 65539 & 99989 & 59715 & 59749 & 4025 I & 99966 & 44 \\
\hline 17 & 35018 & 35029 & 64971 & 99989 & 60033 & 60068 & 39932 & 99966 & 43 \\
\hline 18 & 35578 & 35590 & $6+410$ & 99989 & 60349 & 60384 & 39616 & 99965 & 42 \\
\hline 19 & 36 I 31 & 36143 & 63857 & 99989 & 60662 & 60698 & 39302 & 64 & 41 \\
\hline 20 & $\overline{2} \cdot 36678$ & $\overline{2} \cdot 36689$ & I. 633 I I & $\overline{\mathbf{I}} .99988$ & $\overline{2} .60973$ & $\overline{2} .61009$ & I. 38991 & $\overline{\mathrm{I}} .99964$ & 40 \\
\hline 2 I & 37217 & 37229 & 62771 & 99988 & 61282 & 61319 & 38681 & 99963 & 39 \\
\hline 22 & $3775^{\circ}$ & 37762 & 62238 & 99988 & 61589 & 61626 & $3^{8} 374$ & 99963 & $3^{8}$ \\
\hline 23 & 38276 & 38289 & 6I 7III & 99987 & 61894 & 61 93I & 38069 & 99962 & 37 \\
\hline 24 & $3^{8} 796$ & 38809 & 61191 & 99987 & 62196 & 62234 & 37766 & 99962 & 36 \\
\hline 25 & $\overline{2} .39310$ & $\overline{2} .39323$ & 1. 60677 & $\overline{\mathbf{I}} .99987$ & $\overline{2} .62497$ & $\overline{2} .62535$ & 1. 37465 & $\overline{\mathbf{I}} .99961$ & 35 \\
\hline 26 & 39818 & 39832 & 60168 & 99986 & 62795 & 62834 & 37166 & $9996 \mathrm{I}$ & 34 \\
\hline 27 & 40320 & 40334 & 59666 & 99986 & 63091 & 63131 & 36869 & 99960 & 33 \\
\hline 28 & 40816 & 40830 & 59170 & 99986 & $633^{8} 5$ & 63426 & 36574 & 99960 & $3^{2}$ \\
\hline 29 & 41307 & 41321 & 58679 & 99985 & 63678 & 63718 & 36282 & 99959 & 31 \\
\hline 30 & $\overline{2} \cdot 41792$ & $\overline{2} .41807$ & 1.58193 & $\overline{\mathbf{I}} .99985$ & $\overline{2} .63968$ & $\overline{2} .64009$ & I.35 99I & $\overline{\mathbf{I}} .99959$ & 30 \\
\hline 31 & 42272 & 42287 & 57713 & 99985 & 64256 & 64298 & 35702 & & 29 \\
\hline 32 & 42746 & 42762 & 57238 & 99984 & 64543 & 64585 & $354^{1} 5$ & $9995^{8}$ & 28 \\
\hline 33 & 43216 & 43232 & 56768 & 99984 & 64827 & 64870 & 35130 & 57 & 27 \\
\hline 34 & 43680 & 43696 & 56304 & 99984 & 65110 & $65 \times 54$ & 34846 & 99956 & 26 \\
\hline 35 & $\overline{2} .44139$ & $\overline{2} .44156$ & I. 55844 & $\overline{\mathbf{I}} .99983$ & $\overline{2} .6539 \mathrm{I}$ & $\overline{2} .65435$ & I. 34565 & $\overline{1} .9995^{6}$ & 25 \\
\hline 36 & 44594 & 44611 & 55389 & 99983 & 65670 & 65715 & 34285 & 99955 & 24 \\
\hline 37 & $45 \circ 44$ & 45061 & 54939 & 99983 & 65947 & 65993 & 34007 & 55 & 23 \\
\hline $3^{8}$ & 45489 & 45507 & 54493 & 99982 & 66223 & 66269 & $3373^{I}$ & 99954 & 22 \\
\hline 39 & 45930 & $4594^{8}$ & 54052 & 99982 & 66497 & 66543 & 33457 & 954 & $2 I$ \\
\hline 40 & $\overline{2} \cdot 46366$ & $\overline{2} .46385$ & 1.53615 & $\overline{\mathbf{I}} \cdot 99982$ & $\overline{2} .66769$ & $\overline{2} .66816$ & I. 33184 & $\overline{\mathbf{I}} .99953$ & 20 \\
\hline 41 & 46799 & 46817 & 53183 & 99 98 $\mathbf{r}$ & 67039 & 67087 & $3^{2} 913$ & $9995^{2}$ & 19 \\
\hline 42 & 47226 & 47245 & $5^{2} 755$ & $9998 \mathbf{I}$ & 67308 & 67356 & 32644 & 52 & 18 \\
\hline 43 & 47650 & 47669 & $5^{2} 33^{1}$ & $9998 \mathbf{I}$ & 67575 & 67624 & 32376 & 99951 & 17 \\
\hline 44 & 48069 & 48089 & 51911 & 99980 & $6784 \mathrm{I}$ & 67890 & 32110 & 99 95 I & 16 \\
\hline 45 & $\overline{2} .48485$ & $\overline{2} .48505$ & 1.51495 & $\overline{\mathbf{I}} \cdot 99980$ & $\overline{2} .68104$ & $\overline{2} .68$ I 54 & I. 31846 & $\overline{\mathbf{I}} .99950$ & 15 \\
\hline 46 & 48896 & 48917 & $\begin{array}{lll}5 & 083\end{array}$ & 99979 & 68367 & 68417 & $3^{1} 5^{8} 3$ & 99949 & 14 \\
\hline 47 & 49304 & 49325 & 50675 & 99979 & 68627 & 68678 & $\begin{array}{ll}31 & 322\end{array}$ & 99949 & 13 \\
\hline $4^{8}$ & 49708 & 49729 & 50271 & 99979 & 68886 & 68938 & 31062 & 99948 & 12 \\
\hline 49 & 50108 & 50130 & 49870 & 99978 & 69144 & 69196 & 30804 & 948 & II \\
\hline 50 & $\overline{2} \cdot 50504$ & $\overline{2} .50527$ & I. 49473 & $\overline{\mathbf{x}} .99978$ & $\overline{2} .69400$ & $\overline{2} .69453$ & I. 30547 & г..99 947 & 10 \\
\hline $5^{1}$ & 50897 & 50920 & 49080 & 99 & 69654 & 69708 & 30292 & 99946 & 9 \\
\hline $5^{2}$ & 51287 & 51310 & 48690 & 99977 & 69907 & 69962 & 30038 & 99946 & 8 \\
\hline 53 & 51673 & 51696 & 48304 & 99977 & 70159 & 70214 & 29786 & 99945 & 7 \\
\hline 54 & 52055 & 52079 & 47921 & 99976 & 70409 & 70465 & 29535 & 99 & 6 \\
\hline 55 & $\overline{2} .52434$ & $\overline{2} .52459$ & I. 47541 & $\overline{\mathbf{x}} .99976$ & $\overline{2} .7065^{8}$ & $\overline{2} .70714$ & I .29286 & $\overline{\mathbf{I}} .99944$ & 5 \\
\hline $5^{6}$ & 52810 & 52835 & 47165 & 99975 & 70905 & 70962 & 29038 & 99943 & 4 \\
\hline 57 & 53183 & 53208 & 46792 & 99975 & 71151 & 71208 & 28792 & 99942 & 3 \\
\hline 58 & $5355^{2}$ & $5357^{8}$ & 46422 & 99974 & 71395 & 71453 & 28547 & 99942 & 2 \\
\hline & 53919 & -53945 & 46055 & 99974 & 71638 & 71697 & .28303 & $-9994 \mathrm{I}$ & I \\
\hline \multirow[t]{2}{*}{$60^{\prime}$} & $\overline{2} .54282$ & 2.54308 & 1.45692 & $\overline{\mathbf{1}} .99974$ & $\overline{2} .71880$ & $\overline{2} .71940$ & 1.28060 & $\overline{\mathrm{I}} .99940$ & $0^{\prime}$ \\
\hline & $\log \cos$ & $\log \cot$ & $\log \tan$ & $\log \sin$ & $\log \cos$ & $\log \cot$ & $\log \tan$ & $\log \sin$ & f \\
\hline
\end{tabular}




\begin{tabular}{|c|c|c|c|c|c|c|c|c|c|}
\hline 1 & $\log \sin$ & $\log \tan$ & $\log 00 t$ & $\log c 08$ & $\log \sin$ & $\log \tan$ & $\log \cot$ & $\log \cos$ & \\
\hline 0 & $\overline{2} .71850$ & $\overline{2.71940}$ & 1.25000 & $\overline{1} .99940$ & $\overline{2} .8435^{8}$ & 2.84464 & 1.15536 & $\overline{\mathbf{I}} .99 \mathrm{~S} 94$ & 60 \\
\hline I & 72120 & 1 & & & & & 54 & & 59 \\
\hline 2 & 359 & o & & & & 6 & & & $5^{8}$ \\
\hline 3 & 72597 & 72 & 1 & 023 & 84897 & 85006 & 14994 & 99 S9I & 57 \\
\hline 4 & $72 S_{34}$ & 72 & 27104 & $993^{8}$ & 85075 & 85185 & 14815 & 99891 & $5^{6}$ \\
\hline 5 & $\overline{2} .73069$ & $\overline{2} .731$ & 1.26868 & ז. 99937 & $\overline{2} .85252$ & $\overline{2} .853^{6} 3$ & 1.14637 & '̄.99 S9o & 55 \\
\hline 6 & 73303 & & 26634 & & 85429 & $8554^{\circ}$ & 14460 & $99 \$ 89$ & 54 \\
\hline 7 & 73535 & 73600 & $26+00$ & 99936 & 85 & 85717 & $1428_{3}$ & 888 & 53 \\
\hline 8 & 7 & 73832 & 26 i6s & & 85780 & & 14107 & $99 \$ 87$ & $5^{2}$ \\
\hline 9 & 73497 & 74063 & 25937 & 99934 & 85955 & 069 & 13931 & 86 & $5^{1}$ \\
\hline 10 & $\overline{2} .74226$ & $\overline{2} .74292$ & 1.25708 & โ. .99934 & $\overline{2} .86128$ & $\overline{2} .86243$ & 1.13757 & ז. .99885 & 50 \\
\hline II & 454 & 1 & & & 86301 & & & & 49 \\
\hline 12 & & 74 & 25252 & 2 & 86474 & 86591 & & 99883 & 48 \\
\hline 13 & 74 & 74 & 25026 & & 86 & 86 & & & 47 \\
\hline 14 & 75130 & 75 & $24 \mathrm{SOI}$ & & 86816 & 86935 & 13065 & 99881 & 46 \\
\hline 15 & $\overline{2} .75353$ & $\overline{2} .75423$ & 1.24577 & 1. 99 & $\overline{2} .86987$ & $\overline{2} .87106$ & 1.12894 & I.99 8So & 45 \\
\hline 16 & & 75645 & 24355 & & & & & 79 & 44 \\
\hline 17 & 795 & $75^{8}$ & 24133 & & & 87 & & & 43 \\
\hline is & 015 & 76087 & 23913 & & 87494 & 87616 & 12384 & $99 \mathrm{~S}_{7} 8$ & 42 \\
\hline 19 & 76234 & 76.306 & 23694 & 99927 & 87661 & 87785 & 12215 & 77 & 41 \\
\hline 20 & $\overline{2} \cdot 7^{6}+5^{1}$ & $\overline{2} .7^{6} 525$ & 1.23475 & 1.99926 & $\overline{2} .87829$ & $\overline{2} .87953$ & 47 & ז. 99876 & 40 \\
\hline 21 & 76667 & $767+2$ & & & & & & & 39 \\
\hline 22 & $76 s_{3}$ & 763 & 23042 & 99925 & & 88287 & & 74 & $3^{8}$ \\
\hline 23 & & 77173 & 22827 & 99924 & & & & & 37 \\
\hline 24 & 77310 & 77387 & 22613 & 23 & 90 & & & & 36 \\
\hline 25 & $\overline{2} .77522$ & $\overline{2} .77600$ & I. 22400 & $\overline{\mathbf{I}} .99$ & $\overline{2} .88654$ & $\overline{2} .88$ & 1.11217 & 71 & 35 \\
\hline 26 & 77733 & 77811 & $22 \mathrm{isy}$ & & 88817 & 88 & & & 34 \\
\hline 27 & 77943 & 78022 & $2197^{8}$ & 99921 & $85980^{\circ}$ & 89 iा & & & 33 \\
\hline $2^{2}$ & 78152 & 78232 & 21768 & 20 & 89142 & 89274 & 26 & $6 \hat{S}$ & $3^{2}$ \\
\hline 29 & 78360 & $7^{5} 44^{1}$ & 21559 & 99920 & 89304 & 7 & & 67 & 31 \\
\hline 30 & $\overline{2} .78568$ & $\overline{2} .78649$ & 1.21351 & $\overline{\mathbf{I}} .9$ & $\overline{2} .89464$ & $\overline{2} .8$ & 1.1 & 366 & 30 \\
\hline 31 & $7^{5} 774$ & 78855 & 145 & & 25 & 89 & & 65 & 29 \\
\hline 32 & & $79 \mathrm{C}$ & 39 & & 89784 & & & & 28 \\
\hline 33 & 79183 & 792 & 20734 & & 89943 & & & & 27 \\
\hline 34 & $\begin{array}{r}79386 \\
-\end{array}$ & -79470 & 20530 & 16 & 102 & & 60 & 32 & 26 \\
\hline 35 & $\overline{2} .79588$ & $\overline{2} .79673$ & 1.20327 & $\overline{\mathbf{I}} .99915$ & $\overline{2} .90260$ & $\overline{2} .9$ & 1.09601 & I. .99 & 25 \\
\hline 36 & $79 \div 59$ & 79875 & 20125 & & 90417 & & 43 & & 24 \\
\hline 37. & xyo & 80076 & 24 & & & & & & 23 \\
\hline $3^{8}$ & 80 is9. & So 277 & 19723 & 3 & 730 & & 28 & & 22 \\
\hline 39 & 80385 & 80476 & 19524 & 99912 & $9088_{5}$ & 91029 & 08971 & 57 & 21 \\
\hline 40 & 2. $105^{8} 5$ & $\overline{2} .80674$ & 1.19326 & $\overline{\mathbf{1}} .9$ & 040 & $\overline{2} .91185$ & 1.08815 & ז. 9 & 20 \\
\hline 41 & 80782 & 80872 & 19128 & & 195 & & & & 10 \\
\hline 42 & 80978 & $8 r$ ous & & & & & & & 8 \\
\hline 43 & 81173 & 81 & 36 & & & & & & 7 \\
\hline 44 & 81367 & $8145^{\circ}$ & 541 & & 55 & 03 & 97 & & 16 \\
\hline 45 & 2.81560 & $\overline{2} .8165 .3$ & 1.18347 & $\bar{I} .99907$ & $\overline{2.91 \mathrm{So7}}$ & $\overline{2} .9$ & $1.080_{4} 3$ & $\overline{\mathbf{1}} . \mathrm{g}$ & 15 \\
\hline $4^{6}$ & 81752 & & & & & & & & 14 \\
\hline 47 & 81944 & 82 & 2 & & & & & & 2 \\
\hline 48 & 82134 & 82230 & 0 & & & & & & 12 \\
\hline 49 & 82324 & 82420 & 17580 & 99904 & 92411 & 92565 & 074.35 & 99846 & 11 \\
\hline 50 & 2.82513 & $\overline{2} 82$ & I. & T. & $\overline{2} .92561$ & $\overline{2} .92716$ & $1.072 \mathrm{~S}_{4}$ & i. $.99 \mathrm{~S}_{45}$ & 10 \\
\hline $5 i$ & $8_{2} 701$ & 82709 & & & & & & & \\
\hline $5^{2}$ & 82888 & 82957 & & & & & & & 8 \\
\hline 53 & 83075 & 8375 & 25 & & 07 & & 06835 & $99 \mathrm{~B}_{42}$ & 7 \\
\hline 54 & 83261 & 83361 & 16639 & 90 $\operatorname{seg}$ & 93154 & 93313 & 06687 & 99841 & 6 \\
\hline 55 & $2.8344^{6}$ & $\overline{2} .83547$ & 1.16453 & T. .09 Sys & $\overline{2} .93301$ & $\overline{2} .934^{62}$ & $1.0653^{8}$ & i. $99 \mathrm{~S}_{40}$ & 5 \\
\hline $5^{6}$ & $8,6,30$ & & & $99 \mathrm{~s} 8$ & 93448 & & 06394 & & : \\
\hline 57 & ${ }_{3} \mathrm{SI}_{3}$ & 83416 & 16084 & 9) $\sin 7$ & 93594 & 93 & 06 & $99 \mathrm{~s}_{3} \mathrm{~s}$ & 3 \\
\hline 5 & $S_{3} 90^{\circ}$ & $S_{4} 100$ & & $92 \mathrm{~S} 96$ & & & $06 \times 7$ & & 2 \\
\hline & 84177 & $8_{4} 2 . S_{2}$ & 15718 & Whang & $93 \mathrm{SS}_{5}$ & & $0595 i$ & $99 \$ 36$ & \\
\hline \multirow[t]{2}{*}{60} & 2.84358 & $2.844^{6} 4$ & 1.15536 & 1. $.05 \mathrm{So4}$ & 2.24030 & 2.04105 & $105 \mathrm{Kos}$ & $\mathrm{ims}_{34}$ & 0 \\
\hline & $\log \mathrm{ens}$ & log $\cot$ & $\log \tan$ & $\log \sin$ & log cos & log cot & log $\tan$ & log. sin & \\
\hline
\end{tabular}




\begin{tabular}{|c|c|c|c|c|c|c|c|c|c|}
\hline ' & $\log \sin$ & $\log \tan$ & $\log \cot$ & $\log \cos$ & $\log \sin$ & $\log \tan$ & $\log \cot$ & $\log \cos$ & \\
\hline $0^{\prime}$ & $\overline{2} .94030$ & $\overline{2} .94195$ & $1.05 \bigcirc 05$ & $\overline{1} .99834$ & 1.01923 & $\overline{1} .02162$ & 0.97838 & $\overline{\mathbf{I}} .9976 \mathrm{I}$ & $60^{\prime}$ \\
\hline I & 94174 & 94340 & 05660 & $998_{33}$ & 02043 & 02283 & 97717 & 99760 & 59 \\
\hline 2 & $943^{17}$ & 94485 & 05515 & $99 S_{32}$ & 02163 & 02404 & 97596 & 99759 & $5^{8}$ \\
\hline 3 & $9446 \mathrm{r}$ & 94630 & 05370 & 99831 & 02283 & 02525 & 97475 & 99757 & 57 \\
\hline 4 & 94603 & 94773 & 05227 & $99 \&_{30}$ & 02402 & -02645 & 97355 & 99756 & $5^{6}$ \\
\hline 5 & $\overline{2} .94746$ & $\overline{2} .94917$ & 1.05083 & $\overline{\mathbf{I}} .99 \$ 29$ & $\overline{1} .02520$ & $\overline{1} .02766$ & 0.97234 & $\overline{1} .99755$ & 55 \\
\hline 6 & $94 \mathrm{SS}_{7}$ & 95060 & 04940 & 99828 & 02639 & 02885 & 97115 & 99753 & 54 \\
\hline 7 & 95029 & 95202 & 04798 & 99827 & 02757 & 03005 & 96995 & 99752 & 53 \\
\hline 8 & 95170 & 95344 & 04656 & 99825 & 02874 & 03124 & 96876 & $9975 \mathrm{I}$ & 52 \\
\hline 9 & 95310 & 95486 & $045^{14}$ & 99824 & 02992 & 03242 & $9675^{8}$ & 99749 & 51 \\
\hline 10 & $\overline{2} .95450$ & $\overline{2} .95627$ & 1.04373 & $\overline{\mathbf{I}} .99823$ & I.03 109 & $\overline{1} .0336 \mathrm{I}$ & 0.96639 & I. 99748 & 50 \\
\hline iI & 95589 & 95767 & 04233 & 99822 & 03226 & O3 479 & 96521 & 99747 & 49 \\
\hline 12 & 95728 & 95908 & 04092 & $9982 \mathbf{I}$ & 03342 & O3597 & 96403 & 99745 & 48 \\
\hline 13 & 95867 & 96047 & 03953 & 99820 & $0345^{8}$ & 03714 & 96286 & 99744 & 47 \\
\hline 14 & 96005 & 96187 & 03813 & 99819 & 03574 & $\mathrm{O}_{3} 832$ & 96 I 68 & 99742 & 46 \\
\hline 15 & $\overline{2} .96143$ & $\overline{2} .96325$ & 1.03675 & $\overline{\mathbf{I}} .99817$ & $\overline{\mathrm{I}} .03690$ & $\overline{\mathbf{1}} .03948$ & 0.96052 & $\overline{\mathbf{I}} .9974 \mathrm{I}$ & 45 \\
\hline 16 & 96280 & 96464 & 03536 & 99816 & 03805 & 04065 & 95935 & 99740 & 44 \\
\hline 17 & 96417 & 96602 & 03398 & 99815 & 03920 & $0418 \mathrm{I}$ & 95819 & $9973^{8}$ & 43 \\
\hline 18 & 96553 & 96739 & 03261 & 99814 & 04034 & 04297 & 95703 & 99737 & 42 \\
\hline 19 & 96689 & 96877 & 03123 & 99813 & 04149 & $044^{1} 3$ & $955^{87}$ & 99736 & $4 I$ \\
\hline 20 & $\overline{2} .96825$ & $\overline{2} .97013$ & 1.02987 & $\overline{\mathbf{I}} .998 \mathrm{I} 2$ & $\overline{\mathrm{I}} .04262$ & $\overline{\mathrm{I}} .04528$ & 0.95472 & $\overline{\mathbf{x}} .99734$ & 40 \\
\hline $2 I$ & 96960 & 97150 & 02850 & 99810 & 04376 & 04643 & 95357 & 99733 & 39 \\
\hline 22 & 97095 & 97285 & 02715 & 99809 & 04490 & $0475^{8}$ & 95242 & 99731 & 38 \\
\hline 23 & 97229 & 97421 & 02579 & 99808 & 04603 & 04873 & 95127 & 99730 & 37 \\
\hline 24 & 97363 & 97556 & 02444 & 99807 & 04715 & 04987 & 95013 & 99728 & 36 \\
\hline 25 & $\overline{2} .97496$ & $\overline{2} .9769 \mathrm{I}$ & 1.02309 & $\overline{\mathbf{I}} .99806$ & I. 04828 & $\overline{\mathbf{I}} .05$ Ior & 0.94899 & $\overline{1} .99727$ & 35 \\
\hline 26 & 97629 & 97825 & 02175 & 99804 & 04940 & 05214 & 94786 & 99726 & 34 \\
\hline 27 & 97762 & 97959 & $\mathrm{O}_{2} \mathrm{O}_{4} \mathrm{I}$ & 99803 & 05052 & 05328 & 94672 & 99724 & 33 \\
\hline 28 & 97894 & 98092 & OI 908 & 99802 & 05164 & $0544 \mathrm{I}$ & 94559 & 99723 & 32 \\
\hline 29 & 98026 & 98225 & OI 775 & 99801 & 05275 & 05553 & 94447 & 99721 & 31 \\
\hline 30 & $\overline{2} .98157$ & $\overline{2} .9835^{8}$ & I.01 642 & $\overline{\text { I. }} 99800$ & $\overline{\mathrm{I}} .05386$ & $\overline{\mathbf{I}} .05666$ & 0.94334 & $\overline{\mathbf{I}} .99720$ & 30 \\
\hline $3 \mathrm{I}$ & 98288 & 98490 & OI 510 & 99798 & o5 497 & 05778 & 94222 & 99718 & 29 \\
\hline 32 & 98419 & 98622 & O1 378 & 99797 & 05607 & 05890 & 94110 & 99717 & 28 \\
\hline 33 & 98549 & 98753 & or 247 & 99796 & 05717 & 06002 & 93998 & 99716 & 27 \\
\hline 34 & 98679 & 98884 & or $\times 16$ & 99795 & 05827 & $06 \mathrm{II}_{3}$ & 93887 & 99714 & 26 \\
\hline 35 & $\overline{2} .98808$ & $\overline{2} .99$ oI 5 & I.00 985 & I. 99793 & $\overline{\text { I. }} .05937$ & $\overline{\mathbf{I}} .06224$ & 0.93776 & $\bar{I} .99713$ & 25 \\
\hline 36 & 98937 & 99145 & $\infty 0855$ & 99792 & 06046 & o6 335 & 93665 & 997 I I & 24 \\
\hline 37 & 99066 & 99275 & 00725 & 99791 & 06155 & 06445 & 93555 & 99710 & 23 \\
\hline 38 & 99194 & 99405 & 00595 & 99790 & 06264 & 06556 & 93444 & 99708 & 22 \\
\hline 39 & 99322 & 99534 & 00466 & 99788 & 06372 & 06666 & 93334 & 99707 & 21 \\
\hline 40 & $\overline{2} .99450$ & $\overline{2} .99662$ & 1.00338 & $\overline{\mathbf{I}} .99787$ & $\overline{\mathbf{I}} .0648 \mathrm{I}$ & $\overline{\mathbf{I}} .06775$ & 0.93225 & $\overline{\mathbf{I}} .99705$ & 20 \\
\hline $4^{I}$ & $\overline{2} .99577$ & $\overline{2} .9979$ I & 1.00209 & 99786 & 06589 & 06885 & 93115 & 99704 & 19 \\
\hline 42 & $\overline{2} .99704$ & $\overline{2} .99919$ & $1.0008 I$ & 99785 & 06696 & 06994 & 93006 & 99702 & 18 \\
\hline 43 & $\overline{2} .99830$ & $\overline{1} .00046$ & 0.99954 & 99783 & 06804 & 07103 & 92897 & 99701 & 17 \\
\hline 44 & $\overline{2} .99956$ & $\overline{\mathbf{I}} .00 \mathrm{I} 74$ & 0.99826 & 99782 & 069 II & 07211 & 92789 & 99699 & 16 \\
\hline 45 & $\overline{1} .00082$ & $\overline{1} .00301$ & 0.99699 & $\overline{\mathbf{I}} .9978 \mathbf{I}$ & $\overline{\mathbf{I}} .07018$ & $\overline{1} .07320$ & 0.92680 & '. .99698 & 15 \\
\hline 46 & 00207 & 00427 & 99573 & 99780 & 07124 & 07428 & $9257^{2}$ & 99696 & 14 \\
\hline 47 & 00332 & 00553 & 99447 & $9977^{8}$ & $0723 \mathrm{I}$ & 07536 & 92464 & 99695 & 13 \\
\hline 48 & 00456 & 00679 & 99321 & 99777 & 07337 & 07643 & 92357 & 99693 & 12 \\
\hline 49 & $\infty 5^{8} \mathbf{1}$ & 00805 & 99195 & 99776 & 07442 & 07751 & 92249 & 99692 & II \\
\hline 50 & $\overline{\mathrm{I}} .00704$ & $\overline{\mathbf{I}} .00930$ & 0.99070 & I.99775 & $\overline{\mathrm{I}} .07548$ & โ. $.0785^{8}$ & 0.92142 & $\overline{\mathbf{I}} .99690$ & 10 \\
\hline 51 & 00828 & or 055 & 98945 & 73 & 07653 & 07964 & 92036 & 99689 & 9 \\
\hline 52 & $0095 \mathrm{I}$ & Or 179 & 98821 & 99772 & $0775^{8}$ & 08071 & 91929 & 99687 & 8 \\
\hline 53 & 01074 & ol 303 & 98697 & $9977 \mathrm{I}$ & 07863 & 08177 & 91823 & 99686 & 7 \\
\hline 54 & or 196 & OI 427 & 98573 & 99769 & 07968 & 08283 & 91717 & 99684 & 6 \\
\hline 55 & $\overline{\mathbf{I}} .01318$ & $\overline{1} .01550$ & 0.98450 & $\overline{1} .99768$ & $\overline{\mathrm{I}} .08072$ & $\overline{\mathbf{x}} .08389$ & 0.91611 & $\overline{\mathbf{I}} .99683$ & 5 \\
\hline 56 & or 440 & or 673 & 98327 & 99767 & 08176 & 08495 & 91505 & $9968 \mathrm{I}$ & 4 \\
\hline 57 & or 561 & or 796 & 98204 & 99765 & 08280 & 08600 & 91400 & 99680 & 3 \\
\hline $5^{8}$ & or 682 & O1 918 & 98082 & 99764 & 08383 & 08705 & 91295 & 99678 & 2 \\
\hline & or 803 & _. 02040 & 97960 & $\begin{array}{r}-99763 \\
\end{array}$ & 08486 & 08810 & 91190 & 99677 & I \\
\hline \multirow[t]{2}{*}{$60^{\prime}$} & $\overline{\mathbf{I}} .01923$ & $\overline{1} .02162$ & 0.978 .38 & $\overline{\mathbf{I}} .9976 \mathbf{1}$ & $\overline{\mathbf{1}} .08589$ & $\overline{\mathbf{I}} .08914$ & 0.91086 & $\overline{\mathbf{I}} .99675$ & $0^{\prime}$ \\
\hline & $\log \cos$ & $\log 00 t$ & $\log \tan$ & $\log \sin$ & $\log \cos$ & $\log \cot$ & $\log \tan$ & $\log \sin$ & 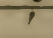 \\
\hline
\end{tabular}




\begin{tabular}{|c|c|c|c|c|c|c|c|c|c|}
\hline ' & $\log \sin$ & $\log \tan$ & $\log \cot$ & $\log \cos$ & $\log \sin$ & $\log \tan$ & $\log \cot$ & $\log \cos$ & \\
\hline $0^{\prime}$ & $\overline{1} .055^{\circ}(y)$ & $\overline{1} .05$ y14 & 0.91050 & $\overline{1} .99675$ & $\overline{1} .1+356$ & $\overline{1} .147$ So & $0 . \$_{5} 220$ & $\overline{1} .99575$ & $60^{\prime}$ \\
\hline 1 & of $(x)^{2}$ & ogorg & 90 y 81 & 99674 & $14+45$ & 14872 & 85128 & 99574 & 59 \\
\hline 2 & os 795 & O9 123 & $90 \$ 77$ & 99672 & 14535 & 14963 & 85037 & 99572 & $5^{8}$ \\
\hline 3 & $08 \$ 97$ & o) 227 & 90773 & 99670 & 14624 & 15054 & 84946 & 99570 & 57 \\
\hline 4 & os 999 & 09330 & 90670 & 99669 & 14714 & 15145 & 84855 & 99568 & $5^{6}$ \\
\hline 5 & $\overline{1} .09101$ & T.og) 434 & 0.90566 & $\overline{1} .99667$ & $\overline{\mathrm{I}} .14 \mathrm{SO}_{3}$ & $\bar{I} .15236$ & 0.84764 & $\overline{\mathbf{I}} .99566$ & 55 \\
\hline 6 & 09 202 & O9 537 & 90463 & 99666 & 14891 & 15327 & 84673 & 99565 & 54 \\
\hline 7 & 09304 & o9 640 & 90360 & 99664 & 14980 & 15417 & 84583 & 99563 & 53 \\
\hline 8 & a) 405 & $0974^{2}$ & $9025^{8}$ & 99663 & 15069 & 15508 & 84492 & & 52 \\
\hline 9 & 09506 & og $\$ 45$ & 90155 & 99661 & 15157 & 15598 & 84402 & 99559 & 51 \\
\hline 10 & I. 09606 & I.09947 & 0.90053 & I. .99659 & $\bar{I} .15245$ & $\overrightarrow{\mathbf{I}} .15688$ & 0.84312 & I. 99557 & 50 \\
\hline 11 & 09707 & 10049 & 89951 & 99658 & 15333 & 15777 & 84223 & 99556 & 49 \\
\hline 12 & a) $\mathrm{SO} 7$ & 10150 & $\mathrm{~S}_{9} \mathrm{~S}_{5} \mathrm{O}$ & 99656 & 15421 & 15867 & 84133 & 99554 & 48 \\
\hline 13 & 099 907 & 10252 & $8974^{8}$ & 99655 & 15508 & 15956 & 84044 & $9955^{2}$ & 47 \\
\hline 14 & 10006 & 10353 & 89647 & 99653 & 15596 & 16046 & 83954 & 99550 & 46 \\
\hline 15 & $\overline{\mathbf{I}} .10106$ & I. 10454 & 0.89546 & $\bar{I} .9965 \mathrm{I}$ & $\overline{1} .15683$ & $\overline{\mathbf{I}} .16135$ & 0.83865 & I. $9954^{8}$ & 45 \\
\hline 16 & 10205 & 10555 & S9 445 & 99650 & 15770 & 16224 & 83776 & 99546 & 44 \\
\hline 17 & 10304 & 10656 & 89344 & 99648 & 15857 & 16312 & 83688 & 99545 & 43 \\
\hline 18 & 10402 & $1075^{6}$ & 89244 & 99647 & 15944 & 16401 & 83599 & 99543 & 42 \\
\hline 19 & 10501 & 10856 & 89144 & 99645 & 30 & 16489 & $835^{11}$ & 99541 & 41 \\
\hline 20 & '. .10599 & $\overline{\mathbf{x}} .10956$ & 0.89044 & $\bar{I} .99643$ & $\overrightarrow{\mathrm{I}} .16116$ & $\overrightarrow{\mathbf{I}} .16577$ & $0.834^{23}$ & $\overline{\mathrm{I}} .99$ & 40 \\
\hline 21 & 10697 & 11056 & 88944 & 99642 & 03 & 16665 & 83335 & & 39 \\
\hline 22 & 10795 & 11155 & 88845 & 99640 & 16289 & 16753 & 83247 & 35 & $3^{8}$ \\
\hline 23 & 10893 & II 254 & 88746 & 99638 & 16374 & 16841 & 83159 & 33 & 37 \\
\hline 24 & 10990 & II 353 & 88647 & 99637 & 16460 & 16928 & 83072 & 99532 & 36 \\
\hline 25 & I.II 1087 & 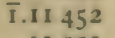 & 0.88548 & $\overline{\mathbf{I}} .99635$ & $\overline{\mathbf{I}} .16545$ & $\overline{\mathbf{I}} .17016$ & 0.82984 & $\overline{\mathrm{I}} .99530$ & 35 \\
\hline 26 & II $1 S_{4}$ & $1155^{1}$ & $88+49$ & 99633 & 16631 & 17103 & $82 S 97$ & 99 & 34 \\
\hline 27 & II 281 & 11649 & 88351 & 99632 & 16716 & 17190 & 82810 & & 33 \\
\hline 28 & II 377 & II $7+7$ & 88253 & 99630 & 16801 & 17277 & 82723 & & $3^{2}$ \\
\hline 29 & II 474 & 11845 & 88155 & 99629 & 86 & 17363 & 37 & & 31 \\
\hline 30 & I.11 570 & T.11 943 & 0.88057 & I. .99627 & $\overline{\mathbf{I}} .16970$ & I. 17450 & $0.8255^{\circ}$ & Iิ.99 & 30 \\
\hline 31 & 11666 & 12040 & 87960 & 99625 & 17055 & 17536 & 82464 & 8 & 29 \\
\hline $3^{2}$ & 11761 & $1213^{8}$ & 87862 & 99624 & 17139 & 17622 & $8237^{8}$ & 17 & 28 \\
\hline 33 & $11 \$_{57}$ & 12235 & 87765 & 99622 & 17223 & 17708 & 82292 & 99515 & 27 \\
\hline 34 & 11952 & 12332 & 87668 & 99620 & 17307 & 17794 & 82206 & 13 & 26 \\
\hline 35 & I.12047 & I. 12428 & $0.8757^{2}$ & $\overline{\mathbf{I}} .99618$ & $\overline{1} .17391$ & $\overline{\mathbf{I}} . \mathbf{1} 7880$ & 0.82 I 20 & $\overline{\mathbf{I}} .99511$ & 25 \\
\hline $3^{6}$ & 12142 & 12525 & 87475 & 99617 & 17474 & 17965 & 82035 & 99509 & 24 \\
\hline 37 & $1223^{6}$ & 12621 & 87379 & 99615 & $1755^{8}$ & 18051 & 949 & 99 & 23 \\
\hline $3^{\mathrm{s}}$ & $123.3^{1}$ & 12717 & 87283 & 99613 & 17641 & 18136 & 81864 & 25 & 22 \\
\hline 39 & 12425 & 12813 & 87187 & 99612 & 17724 & 18221 & 81779 & 3 & 21 \\
\hline 40 & $\overline{1} .12 ; 19$ & $\overline{1} .12909$ & 0.87091 & $\overline{1} .99610$ & T..17 807 & T.18 306 & 0.81604 & I. 99 & 20 \\
\hline 41 & 126,12 & 13004 & 86996 & 608 & 17800 & 18391 & $81(10 x)$ & 199 & 19 \\
\hline 42 & 12706 & 13099 & 86901 & 99607 & 17973 & 18475 & 81525 & 99497 & 18 \\
\hline 43 & 12799 & 13194 & 86806 & 99605 & 55 & 18560 & 81440 & 99495 & 17 \\
\hline 44 & $12 S_{9} 2$ & 13289 & $867^{11}$ & 99603 & 18137 & 18644 & 81356 & 94 & 6 \\
\hline 45 & $\overline{1} .12085$ & T.13384 & 0.86616 & $\overline{1} .99601$ & $\bar{I} .18220$ & T.18 728 & 0.81272 & I. .99492 & 15 \\
\hline 46 & 13078 & $1347^{8}$ & 86522 & 99600 & 18,302 & $18 \$ 12$ & 81188 & 99490 & 14 \\
\hline 47 & 13171 & 13573 & 86427 & 99598 & $18_{3} s_{3}$ & $18 \mathrm{Sig} 6$ & 81104 & 99485 & 13 \\
\hline 48 & 1326,3 & 13667 & 86333 & 99596 & 18465 & 18979 & 81021 & 99456 & 12 \\
\hline 49 & 13355 & 13761 & 86239 & 99595 & 18547 & 19063 & 80937 & $994^{8} 4$ & II \\
\hline 50 & I. $13+47$ & 7.13854 & 0.86146 & ז..99593 & T.1S6,28 & T.19 146 & o.So $S_{54}$ & T. $994 \mathrm{~S}_{2}$ & 10 \\
\hline 51 & 135.30 & 1,3948 & 86052 & & 18709 & 19229 & So 771 & 99450 & (0) \\
\hline $5^{2}$ & 13630 & 14041 & 85059 & 99589 & 18790 & 19312 & 88 & 99475 & 8 \\
\hline 53 & 13722 & 14134 & 85856 & 995 Ss & 18871 & 19) 395 & 80605 & $9947^{6}$ & 7 \\
\hline 54 & 1,3813 & 14227 & 85773 & 99586 & IS952 & $1947^{8}$ & 80522 & 99474 & 6 \\
\hline 55 & T.1.3004 & 7.14320 & 0.856 .80 & T.99 $5^{8.4}$ & T.19033 & $\overline{1} .19561$ & 0.804 .39 & 1.99472 & 5 \\
\hline $5^{6}$ & $13 \% 94$ & 14412 & $855 \mathrm{sS}$ & 99582 & 89113 & 19643 & So 357 & $9947^{\circ}$ & 4 \\
\hline 57 & $1400_{5}$ & 14504 & $\$_{5} 496$ & 99 $5^{81}$ & 19193 & 19) 725 & 80275 & 99465 & 3 \\
\hline $5^{8}$ & 14175 & 145127 & 85403 & 99579 & 19273 & $19 \mathrm{Sol}$ & 80193 & 99466 & 2 \\
\hline 59 & 14266 & 146.58 & 85312 & 99577 & 10. 353 & 19850 & So 118 & 92464 & 1 \\
\hline \multirow[t]{2}{*}{$60^{\prime}$} & I.1.4356 & 1.14750 & 0.55220 & i. 00575 & 1.11433 & $\overline{1} .109-1$ & 0.80020 & 1.90462 & $0^{\prime}$ \\
\hline & $\log 008$ & $\operatorname{lng} \cot$ & $\log \tan$ & $\log \sin$ & $\log \cos$ & $\log \mathrm{cot}$ & $\log \operatorname{lan}$ & $\log \sin$ & 1 \\
\hline
\end{tabular}




\begin{tabular}{|c|c|c|c|c|c|c|c|c|c|}
\hline ' & $\log \sin$ & $\log \tan$ & $\log \cot$ & $\log 008$ & $\log \sin$ & $\log \tan$ & $\log \cot$ & $\log 008$ & \\
\hline $0^{\prime}$ & $\overline{1} .19433$ & $\overline{1} .1997 \mathrm{I}$ & 0.80029 & $\overline{1} .99462$ & $\overline{1} .23967$ & $\overline{\mathrm{I}} .24632$ & 0.75368 & ז̄.99335 & $60^{\prime}$ \\
\hline 1 & 19513 & 20053 & 79947 & 99460 & $2+039$ & 24706 & 75294 & 99333 & 59 \\
\hline 2 & 19592 & 20134 & 79866 & $9945^{8}$ & 24110 & 24779 & 75221 & 99331 & $5^{8}$ \\
\hline 3 & 19072 & 20216 & 79784 & 99456 & $2418 I$ & 24853 & 75147 & 99328 & $\begin{array}{l}57 \\
56\end{array}$ \\
\hline 4 & $1975^{1}$ & 20297 & 79703 & 99454 & 24253 & 24926 & 75074 & 99326 & 56 \\
\hline 5 & $\overline{1} .19 \$_{30}$ & $\overline{1} .2037^{8}$ & 0.79622 & $\overline{1} .9945^{2}$ & $\overline{\mathrm{I}} .24324$ & $\overline{\mathbf{1}} .25000$ & 0.75000 & $\overline{\mathbf{I}} .99324$ & 55 \\
\hline 6 & 19909 & 20459 & 79541 & 99450 & 24395 & 25073 & 74927 & 99322 & 54 \\
\hline 7 & 19985 & 20540 & 79460 & $9944^{8}$ & 24466 & 25146 & 74854 & 99319 & 53 \\
\hline 8 & 20067 & 20621 & 79379 & 99446 & 24536 & 25219 & 74781 & 99317 & $5^{2}$ \\
\hline 9 & 20145 & 20701 & 79299 & 99444 & 24607 & 25292 & 74708 & 99315 & $5^{1}$ \\
\hline 10 & T. 20223 & $\overline{\mathrm{I}} .20782$ & 0.79218 & $\overrightarrow{\mathrm{I}} .99442$ & $\overline{\mathrm{I}} .24677$ & $\overline{\mathbf{I}} .25365$ & 0.74635 & I.99313 & 50 \\
\hline II & 20302 & 20862 & 79138 & 99440 & 24748 & 25437 & $745^{6} 3$ & 99310 & 49 \\
\hline 12 & $203 \mathrm{SO}$ & 20942 & $79 \circ 5^{8}$ & $9943^{8}$ & 24818 & 25510 & 74490 & 99308 & 48 \\
\hline 13 & $2045^{8}$ & 21022 & $7^{8} 97^{8}$ & 99436 & 24888 & 25582 & 74418 & 99306 & 47 \\
\hline 14 & 20535 & 21102 & $7^{8898}$ & 99434 & $2495^{8}$ & 25655 & 74345 & 99304 & 46 \\
\hline 15 & $\overline{1} .20613$ & $\overline{\mathrm{I}} .21 \mathrm{I} 82$ & 0.78818 & $\overline{\mathbf{I}} .99432$ & $\overline{\mathrm{I}} .25028$ & $\overline{\mathrm{I}} .25727$ & 0.74273 & $\overline{\mathrm{I}} .9930 \mathrm{I}$ & 45 \\
\hline 16 & 20691 & 21261 & 78739 & 99429 & 25098 & 25799 & 74201 & 99299 & 44 \\
\hline 17 & 20768 & 21341 & 78659 & 99427 & 25168 & 25871 & 74129 & 99297 & 43 \\
\hline 18 & 20845 & 21420 & 78580 & 99425 & 25237 & 25943 & 74057 & 99294 & $4^{2}$ \\
\hline 19 & 20922 & 21499 & 78501 & 99423 & 25307 & 26015 & 73985 & 99292 & $4 \mathrm{I}$ \\
\hline 20 & İ.20 999 & $\overrightarrow{\mathrm{I}} \cdot 21578$ & 0.78422 & $\bar{I} .99421$ & $\overline{\mathbf{I}} .25376$ & $\overline{\mathbf{I}} .26086$ & 0.73914 & $\overline{\mathbf{I}} .99290$ & 40 \\
\hline 21 & 21076 & 21657 & 78343 & 99419 & 25445 & $2615^{8}$ & 73842 & 99288 & 39 \\
\hline 22 & 21153 & 21736 & 78264 & 99417 & 25514 & 26229 & 73771 & 99285 & $3^{8}$ \\
\hline 23 & 21229 & 21814 & 78186 & 99415 & $255^{83}$ & 26301 & 73699 & 99283 & 37 \\
\hline 24 & 21306 & 21893 & 78107 & $994 \mathbf{1} 3$ & 25652 & 26372 & 73628 & 99281 & 36 \\
\hline 25 & $\overline{\mathrm{I}} .2 \mathrm{I} 382$ & $\overline{\mathrm{I}} .2197 \mathrm{I}$ & 0.78029 & $\overline{\mathbf{I}} .994$ I I & $\overline{\mathrm{I}} .2572 \mathrm{I}$ & $\overline{\mathbf{I}} .26443$ & 0.73557 & $\overline{\mathrm{I}} .99278$ & 35 \\
\hline 26 & $2145^{8}$ & 22049 & 77951 & 99409 & 25790 & 26514 & 73486 & 99276 & 34 \\
\hline 27 & 21 534 & 22127 & 77873 & 99407 & $25^{8} 5^{8}$ & $265^{85}$ & 73415 & 99274 & 33 \\
\hline 28 & 21610 & 22205 & 77795 & 99404 & 25927 & 26655 & 73345 & 99271 & 32 \\
\hline 29 & 21685 & 22283 & 77717 & 99402 & 25995 & 26726 & 73274 & 269 & 31 \\
\hline 30 & $\overline{\mathrm{I}} .2176 \mathrm{I}$ & $\overline{\mathrm{I}} .2236 \mathrm{I}$ & 0.77639 & $\overline{\mathbf{I}} .99400$ & $\overline{\mathbf{I}} .26063$ & $\overrightarrow{\mathbf{I}} .26797$ & 0.73203 & $\overline{\mathbf{1}} .99267$ & 30 \\
\hline 31 & 21836 & $2243^{8}$ & $775^{62}$ & 99398 & 26 I 31 & 26867 & 73133 & 99264 & 29 \\
\hline $3^{2}$ & 21912 & $225^{16}$ & 77484 & 99396 & 26199 & 26937 & 73063 & 99262 & 28 \\
\hline 33 & 21987 & 22593 & 77407 & 99394 & 26267 & 27008 & 72992 & 99260 & 27 \\
\hline 34 & 22062 & 22670 & 77330 & 99392 & 26335 & 27078 & 72922 & 99257 & 26 \\
\hline 35 & $\overline{\mathrm{I}} .22137$ & $\overline{\mathbf{I}} .22747$ & 0.77253 & I.99390 & $\overline{\mathrm{I}} .26403$ & $\overline{\mathrm{I}} .27148$ & 0.72852 & $\overline{\mathrm{I}} .99255$ & 25 \\
\hline 36 & 22211 & 22824 & 77176 & 99388 & 26470 & 27218 & 72782 & 99252 & 24 \\
\hline 37 & 22286 & 22901 & 77099 & 99385 & $2653^{8}$ & 27288 & 72712 & 99250 & 23 \\
\hline 38 & 22361 & 22977 & 77023 & 99383 & 26605 & 27357 & 72643 & 99248 & 22 \\
\hline 39 & 22435 & 23054 & 76946 & 99381 & 26672 & 27427 & $7^{2} 573$ & 99245 & 21 \\
\hline 40 & $\overline{\mathbf{1}} .22509$ & $\overline{\mathrm{I}} .23130$ & 0.76870 & 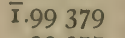 & $\overline{\mathrm{I}} .26739$ & $\overrightarrow{\mathbf{I}} .27496$ & 0.72504 & $\overrightarrow{\mathbf{I}} .99243$ & 20 \\
\hline 41 & 22583 & 23206 & 76794 & 77 & 26806 & $275^{66}$ & 72434 & $9924 \mathrm{I}$ & 19 \\
\hline 42 & 22657 & 23283 & 76717 & 99375 & 26873 & 27635 & 72365 & $9923^{8}$ & 18 \\
\hline 43 & 22731 & 23359 & 76641 & 99372 & 26940 & 27704 & 72296 & 99236 & 17 \\
\hline 44 & 22805 & 23435 & 76565 & 99370 & 27007 & 27773 & 72227 & 99233 & 16 \\
\hline 45 & $\overline{\text { I. }} 22878$ & $\overline{\mathrm{I}} .23510$ & 0.76490 & $\overline{\mathrm{I}} .99368$ & $\overline{1} .27073$ & $\overline{\mathrm{I}} .27842$ & $0.7^{2} 15^{8}$ & $\overline{\mathbf{I}} .9923 \mathrm{I}$ & 15 \\
\hline 46 & 22952 & 23586 & 76414 & 99366 & 27140 & 27911 & 72089 & 99229 & 14 \\
\hline 47 & 23025 & 23661 & 76339 & 99364 & 27206 & 27980 & 72020 & 99226 & 13 \\
\hline 48 & 23098 & 23737 & 76263 & 99362 & 27273 & 28049 & 71951 & 99224 & 12 \\
\hline 49 & 23171 & 23812 & 76188 & 99359 & 27339 & 28117 & 71883 & $9922 \mathrm{I}$ & II \\
\hline 50 & $\overline{\mathrm{I}} .23244$ & $\overline{\mathrm{I}} .23887$ & 0.76 II 3 & $\overline{\mathbf{I}} .99357$ & $\overline{\mathrm{I}} .27405$ & $\overline{\mathbf{x}} .28186$ & 0.71814 & $\overline{\mathbf{I}} .99219$ & 10 \\
\hline $5^{1}$ & 23317 & 23962 & 76038 & 99355 & $2747 \mathrm{I}$ & 28254 & 71746 & 99217 & 9 \\
\hline $5^{2}$ & 23390 & $24 \circ 37$ & 75963 & 99353 & 27537 & 28323 & 71677 & 99214 & 8 \\
\hline 53 & 23462 & 24112 & 75888 & $9935^{1}$ & 27602 & 28391 & 71609 & 99212 & 7 \\
\hline 54 & 23535 & 24186 & 75814 & 99348 & 27668 & 28459 & 71541 & 99209 & 6 \\
\hline 55 & $\overline{\mathrm{I}} .23607$ & $\overline{\mathrm{I}} .2426 \mathrm{I}$ & 0.75739 & $\overline{\mathrm{I}} .99346$ & $\overrightarrow{\mathrm{I}} .27734$ & $\overline{\mathrm{I}} .28527$ & 0.71473 & $\overline{\mathrm{I}} .99207$ & 5 \\
\hline $5^{6}$ & 23679 & 24335 & 75665 & 99344 & 27799 & 28595 & 71405 & 99204 & 4 \\
\hline 57 & $2375^{2}$ & 24410 & 75590 & $9934^{2}$ & 27864 & 28662 & $7^{1} 33^{8}$ & 99202 & 3 \\
\hline $5^{8}$ & 23823 & 24484 & $755^{16}$ & 99340 & 27930 & 28730 & 71270 & 99200 & 2 \\
\hline & 23895 & $2455^{8}$ & 75442 & 99337 & 27995 & 28798 & 71202 & 99197 & I \\
\hline \multirow{2}{*}{$60^{\prime}$} & ธ. 23967 & $\overline{\mathrm{I}} .24632$ & 0.75368 & $\overline{\mathrm{x}} .99335$ & I. 28060 & $\overline{\mathbf{I}} .28865$ & 0.71135 & $\overline{\mathbf{I}} .99 \mathbf{1 9 5}$ & $0^{\prime}$ \\
\hline & $\log \cos$ & $\log \cot$ & $\log \tan$ & $\log \sin$ & $\log \mathrm{cos}$ & $\log \cot$ & $\log \tan$ & $\log \sin$ & 1 \\
\hline
\end{tabular}




\begin{tabular}{|c|c|c|c|c|c|c|c|c|c|}
\hline ' & $\log \sin$ & $\log \tan$ & $\log \cot$ & $\log 608$ & $\log \sin$ & $\log \tan$ & $\log \cot$ & $\log c 08$ & \\
\hline $0^{\prime}$ & $\overline{1} .25060$ & $\overline{1} .28 \mathrm{Su}_{5}$ & 0.71135 & $\overline{1} .99195$ & $\overline{1} .31 .758$ & $\overline{\mathrm{I}} \cdot 32747$ & 0.67253 & $\overline{1} .990 .40$ & $60^{\prime}$ \\
\hline 1 & 28125 & 28933 & 71067 & 99192 & 31847 & 32510 & 67 190 & $99<38$ & 59 \\
\hline 2 & 28190 & 29000 & 71000 & 99190 & 31907 & 32872 & 67128 & 99035 & $5^{8}$ \\
\hline 3 & 28254 & 29067 & 70933 & 99187 & 31966 & $3^{2} 933$ & 67067 & 99032 & 57 \\
\hline 4 & 28319 & 29134 & 70566 & 99 IS 5 & 32025 & 32995 & 67005 & 99030 & $5^{6}$ \\
\hline 5 & $\overline{\mathrm{I}} .2 \mathrm{~S}_{3} \mathrm{~S}_{4}$ & $\overline{\mathrm{I}} .2920 \mathrm{I}$ & 0.70799 & $\overline{\mathrm{I}} .99 \mathrm{ISz}$ & I. $3200_{4}$ & $\overline{\mathrm{i}} \cdot 33057$ & 0.66943 & T. .99027 & 55 \\
\hline 6 & 25448 & 29268 & 70732 & 99 1So & 32143 & 33119 & 66881 & 99024 & \\
\hline 7 & 28512 & 29335 & 70665 & 99177 & 32202 & 33180 & 66820 & & 53 \\
\hline 8 & 28577 & 29402 & 70598 & 99175 & $3226 \mathrm{I}$ & 33242 & $6675^{8}$ & 99019 & $5^{2}$ \\
\hline 9 & 28641 & $29+65$ & 70532 & 99172 & $3^{2} 3^{19}$ & 33303 & 97 & 16 & $5^{1}$ \\
\hline 10 & T. 28705 & $\overline{1} .29535$ & 0.70465 & $\overline{1} .99170$ & $\overline{1} \cdot 32378$ & $\overline{\mathbf{x}} \cdot 33365$ & 0.66635 & I. 99013 & 50 \\
\hline 11 & 28769 & 29601 & 70399 & 99167 & 32437 & 33426 & 66574 & 11 & 49 \\
\hline 12 & 28833 & 29668 & 70332 & 99165 & 32495 & $33+87$ & $665^{13}$ & 99008 & 48 \\
\hline 13 & 25596 & 29734 & 70266 & 99162 & 32553 & $3354^{8}$ & $6645^{2}$ & 99005 & 47 \\
\hline 14 & $2 S 960$ & 29800 & 70200 & 99160 & 32612 & 33609 & 66391 & 02 & 46 \\
\hline 15 & İ.29024 & '̄. 29866 & 0.70134 & $\overline{\mathbf{I}} .99157$ & $\overline{1} .32670$ & $\overline{\mathbf{I}} \cdot 33670$ & 0.66330 & $\overline{\mathbf{I}} .99000$ & 45 \\
\hline 16 & 29087 & 29932 & 70068 & 99155 & 32728 & 33731 & 66269 & 98997 & 44 \\
\hline 17 & 29150 & $2999 \mathrm{~S}$ & 70002 & $99 \times 52$ & $3^{2} 7^{86}$ & 33792 & 66208 & 98994 & 43 \\
\hline 18 & 29214 & 30064 & 69936 & 99150 & $32 S_{44}$ & $33 S_{53}$ & 66147 & 98991 & $4^{2}$ \\
\hline 19 & 29277 & 301 & 69870 & 7 & $\mathrm{O} 2$ & 3 & 7 & 9 & $4 I$ \\
\hline 20 & $\overline{\mathrm{I}} .29340$ & $\overline{1} .30195$ & $0.69 \mathrm{SO}_{5}$ & '. 99145 & I. 32960 & $\overline{\mathbf{I}} \cdot 33974$ & 0.66026 & T. 98986 & 40 \\
\hline 21 & 29403 & $3026 i$ & 69739 & $99 \mathbf{I}_{4} 2$ & 33 or 8 & 34034 & 65966 & $989^{8} s_{3}$ & 39 \\
\hline 22 & 29466 & 30326 & 69674 & 99 I 40 & 33075 & 34095 & 65905 & $9 S 980$ & $3^{8}$ \\
\hline 23 & 29529 & 30391 & 69609 & 99137 & 33133 & 34155 & 65845 & $9897^{8}$ & 37 \\
\hline 24 & $2959 \mathrm{I}$ & 30457 & 69543 & 99135 & 33190 & 34215 & $657 S_{5}$ & $9 S 975$ & 36 \\
\hline 25 & $\overline{\mathrm{I}} .29654$ & $\overline{1} .30522$ & $0.6947^{8}$ & $\overline{1} .99132$ & $\overline{\mathbf{I}} \cdot 332.48$ & $\overline{\mathrm{I}} \cdot 34276$ & 0.65724 & 1. 98972 & 35 \\
\hline 26 & 29716 & 30587 & $69+13$ & 99 I 30 & 33305 & 34336 & 65664 & 98969 & 34 \\
\hline 27 & 29779 & 30652 & $6934^{8}$ & 99127 & 33362 & 34396 & 65604 & 98967 & 33 \\
\hline 28 & 29841 & 30717 & 69283 & 99124 & 33420 & 34456 & 65544 & 98964 & 32 \\
\hline 29 & 29903 & 30782 & 69218 & 99122 & 33477 & 34516 & 65484 & 98961 & 31 \\
\hline 30 & $\overline{\mathrm{I}} .29966$ & $\overline{\text { I. }} \cdot 30846$ & 0.69154 & $\overline{\mathrm{I}} .99119$ & $\overline{\mathrm{I}} \cdot 33534$ & $\overline{\mathbf{I}} \cdot 3457^{6}$ & 0.65424 & $\overline{1} .9895 \mathrm{~s}$ & 30 \\
\hline 31 & 30028 & 30911 & 69089 & 99117 & 33591 & 34635 & 65365 & 98955 & 29 \\
\hline 32 & 30090 & 30975 & 69025 & $99 \mathrm{II}_{4}$ & 33647 & 34695 & 65305 & 98953 & 28 \\
\hline 33 & 30151 & 31040 & 68960 & 99112 & 33704 & 34755 & 65245 & 98950 & 27 \\
\hline 34 & 30213 & 31104 & $6 \$ \$ 96$ & 99109 & 33761 & 34814 & 65186 & 99947 & 26 \\
\hline 35 & i. 30275 & ‥31 168 & $0.68 \$_{32}$ & $\overline{\mathrm{I}} .99106$ & $\overline{1} .33818$ & $\overline{1} .34874$ & $0.65+26$ & $\overline{1} .98944$ & 25 \\
\hline 36 & 30.3 .36 & 31233 & 68767 & 99104 & 33874 & 34933 & 65067 & 98941 & 24 \\
\hline 37 & $3039 \mathrm{~s}$ & 31297 & 68703 & 99101 & 33931 & $3+992$ & 65008 & $9893^{8}$ & 23 \\
\hline 38 & 30459 & 31361 & 68639 & 99099 & 33987 & 35051 & 64949 & 98936 & 22 \\
\hline 39 & 30521 & 31425 & 68575 & 99096 & 34043 & 35111 & 64859 & 98933 & 21 \\
\hline 40 & $\overline{1} \cdot 305^{82}$ & $\overline{1} \cdot 31489$ & 0.68511 & $\overline{1} .99093$ & '. 34100 & $\overline{\mathrm{I}} .35170$ & 0.64830 & $\overline{1} .98930$ & 20 \\
\hline 41 & 30643 & 31552 & 68448 & 99 og) 1 & 34156 & 35229 & 64771 & 98927 & 19 \\
\hline 42 & 30704 & 31616 & 68384 & 99088 & 34212 & 35288 & 64712 & 95924 & 18 \\
\hline 43 & $307^{65}$ & 31679 & 68321 & $9 y 086$ & 34268 & 35347 & 64653 & 98921 & 17 \\
\hline 44 & $30 \$ 26$ & 31743 & $6 S 257$ & $990_{3}$ & 34324 & 35405 & 64595 & $9^{8} 919$ & 16 \\
\hline 45 & ‥ 30887 & T. 31806 & 0.68194 & I. .99 oso & T. 343 so & i. 35464 & $0.6453^{6}$ & T.98916 & 15 \\
\hline 46 & 30947 & 31870 & 68130 & 99078 & 34436 & 35523 & 64477 & $982_{3}$ & 14 \\
\hline 47. & 31008 & 3193.3 & 68067 & 99075 & 34491 & 35 & 64419 & yS910 & 13 \\
\hline $4^{8}$ & 31068 & $3^{1} 99^{6}$ & 68004 & 99072 & 34547 & 35640 & 64360 & 98907 & 12 \\
\hline 49 & $31+29$ & 32059 & 67941 & 99070 & 34602 & $35 \mathrm{bogs}$ & 64302 & c5 904 & 11 \\
\hline 50 & ‥3 3189 & T. 32122 & 0.67878 & I. .99067 & $\overline{1} .34658$ & ‥35 757 & 0.64243 & T.0S 001 & 10 \\
\hline $5 !$ & 31250 & 32185 & 67815 & 99064 & 34713 & 35815 & 64185 & 98.98 & 9 \\
\hline $5^{2}$ & 31310 & $3224^{8}$ & 67752 & 9906,2 & $3+7^{(x)}$ & 35873 & 64127 & $95 \mathrm{sg} 6$ & 8 \\
\hline 53 & 31.370 & 32311 & 67689 & 99059 & $34 \times 24$ & 35931 & $6406 x$ & $28 \mathrm{So3}$ & 7 \\
\hline 54 & 314,30 & 32373 & 67627 & 99056 & 34879 & 35989 & 64011 & ys Sigo & 6 \\
\hline 55 & $\bar{T} \cdot 31 \cdot 480$ & T. 32436 & 0.67564 & i. 90054 & T. 34934 & T. 36047 & 0.63953 & T.gS SS7 & 5 \\
\hline 56 & 31549 & $324^{18} 8$ & 67502 & $000=1$ & 34959 & 36105 & 63595 & 95854 & 4 \\
\hline 57. & $31(x(x)$ & 32564 & 674,39 & $9904 \mathrm{~s}$ & 35044 & 36163 & 63837 & $9 \mathrm{SSSt}$ & 3 \\
\hline $5^{8}$ & $316(x)$ & 32623 & 67377 & 90.46 & 35 ond & 36221 & 63779 & 08878 & 2 \\
\hline$=0$ & 31728 & $3265_{5}$ & 67.315 & 9904.3 & .35154 & 30279 & 63721 & $9 \times 875$ & 1 \\
\hline \multirow[t]{2}{*}{$60^{\prime}$} & $1.31-5.5$ & 1.32747 & 0.67253 & I.00 0.90 & I. 35200 & $1 . ; 6336$ & 0.6366 .4 & $1.085,2$ & $0^{\prime}$ \\
\hline & $\log \cos$ & $\log \cot t$ & $\log \tan$ & log $\sin$ & $\log \cos$ & $\log \cot$ & $\log \tan$ & log $\sin$ & \\
\hline
\end{tabular}




\begin{tabular}{|c|c|c|c|c|c|c|c|c|c|}
\hline ' & $\log \sin$ & $\log \tan$ & $\log \cot$ & $\log \cos$ & $\log \sin$ & $\log \tan$ & $\log \cot$ & $\log \cos$ & \\
\hline $0^{\prime}$ & $\overline{1} .35209$ & $\overline{\mathbf{1}} .36336$ & 0.63064 & $\overline{1} .98572$ & $\overline{1} \cdot 3^{S} 368$ & $\overline{1} .39677$ & 0.60323 & $\overline{1} .98600$ & $60^{\prime}$ \\
\hline I & 35263 & 36394 & 63606 & 98869 & 38418 & $3973 \mathbf{I}$ & 60269 & 98687 & 59 \\
\hline 2 & 35318 & 36452 & 63548 & 98867 & 38469 & 39785 & 60215 & 98684 & 58 \\
\hline 3 & 35373 & 36509 & 63491 & 98864 & $3^{8} 5^{19}$ & $39 \mathrm{~S}_{3} \mathrm{~s}$ & 60162 & $9868 \mathrm{i}$ & 57 \\
\hline 4 & 35427 & 36566 & 63434 & $9886 \mathbf{I}$ & 38570 & 39892 & 60108 & 98678 & 56 \\
\hline 5 & $\overline{\mathbf{I}} \cdot 3548 \mathrm{I}$ & $\overline{\mathrm{I}} \cdot 36624$ & 0.63376 & $\overline{\mathbf{I}} .988_{5} 8$ & $\overline{\mathbf{1}} \cdot 39620$ & $\overline{\mathbf{I}} .39945$ & 0.60055 & $\overline{\mathrm{I}} .98675$ & 55 \\
\hline 6 & 35536 & $3668 \mathrm{i}$ & 63319 & $98 S_{55}$ & 38670 & 39999 & 60001 & $9867 \mathbf{I}$ & 54 \\
\hline 7 & 35590 & $3673^{8}$ & 63262 & 98852 & $3^{8} 721$ & 40052 & 59948 & 98668 & 53 \\
\hline 8 & 35644 & $3^{6} 795$ & 63205 & 98849 & $3^{8} 77^{1}$ & 40106 & 59894 & 98665 & 52 \\
\hline 9 & 35698 & 36852 & 63148 & 98846 & 38821 & 40159 & 59841 & 98662 & 51 \\
\hline 10 & $\overline{\mathbf{1}} \cdot 35752$ & $\overline{\mathbf{I}} \cdot 36909$ & 0.63091 & $\overline{\mathbf{I}} .98843$ & $\overline{\mathbf{I}} \cdot 3887 \mathbf{I}$ & $\overline{1} .40212$ & 0.59788 & I. 98659 & 50 \\
\hline II & 35806 & 36966 & 63034 & 98840 & $3892 \mathbf{I}$ & 40266 & 59734 & 98656 & 49 \\
\hline 12 & 35860 & 37023 & 62977 & 98837 & $3^{8} 971$ & 40319 & $5968 \mathrm{I}$ & 98652 & 48 \\
\hline 13 & 35914 & 37080 & 62920 & 98834 & 39021 & 40372 & 59628 & 98649 & 47 \\
\hline 14 & 35968 & 37137 & 62863 & $988_{3} \mathrm{I}$ & 39071 & 40425 & 59575 & 98646 & 46 \\
\hline 15 & $\overline{\mathrm{I}} \cdot 36022$ & $\overline{\mathrm{I}} \cdot 37193$ & 0.62807 & $\overline{\mathrm{I}} .98828$ & $\overline{\mathrm{I}} \cdot 3912 \mathrm{I}$ & $\overline{\mathbf{I}} .40478$ & 0.59522 & $\overline{\mathbf{I}} .98643$ & 45 \\
\hline 16 & 36075 & 37250 & 62750 & 98825 & 39170 & $4053 \mathrm{I}$ & 59469 & 98640 & 44 \\
\hline 17 & 36129 & 37306 & 62694 & 98822 & 39220 & 40584 & 59416 & 98636 & 43 \\
\hline 18 & 36182 & 37363 & 62637 & 98819 & 39270 & 40636 & 59364 & 98633 & 42 \\
\hline 19 & 36236 & 37419 & $625^{8 i}$ & 98816 & 39319 & 40689 & 59311 & 98630 & 41 \\
\hline 20 & $\overline{\mathrm{I}} \cdot 36289$ & $\overline{\mathbf{r}} \cdot 37476$ & 0.62524 & $\overline{\mathbf{I}} .988_{\mathbf{I}} 3$ & $\overline{\mathbf{I}} \cdot 39369$ & $\overline{\mathbf{I}} .40742$ & $0.5925^{8}$ & $\overline{1} .98627$ & 40 \\
\hline $2 I$ & 36342 & 37532 & 62468 & 98810 & 39418 & 40795 & 59205 & 98623 & 39 \\
\hline 22 & 36395 & $375^{88}$ & 62412 & 98807 & 39467 & 40847 & 59153 & 98620 & 38 \\
\hline 23 & 36449 & 37644 & 62356 & 98804 & 39517 & 40900 & 59100 & 98617 & 37 \\
\hline 24 & 36502 & 37700 & 62300 & $9880 \mathrm{I}$ & 39566 & 40952 & 59048 & $986 I_{4}$ & 36 \\
\hline 25 & $\overline{\mathbf{I}} .36555$ & $\overline{\mathrm{I}} \cdot 37756$ & 0.62244 & $\overline{\mathbf{I}} .98798$ & $\overline{\mathrm{r}} \cdot 39615$ & $\overline{\mathbf{I}} .4 \mathrm{I} .005$ & 0.58995 & $\overline{\mathbf{I}} .98610$ & 35 \\
\hline 26 & 36608 & 37812 & 62188 & 98795 & 39664 & 41057 & 58943 & 98607 & 34 \\
\hline 27 & 36660 & 37868 & 62132 & 98792 & 39713 & 41109 & 58891 & 98604 & 33 \\
\hline 28 & 36713 & 37924 & 62076 & 98789 & 39762 & 41161 & 58839 & 98601 & 32 \\
\hline 29 & 36766 & 37980 & 62020 & 98786 & $398 \mathrm{rI}$ & $4 I 2 I 4$ & $5^{8} 786$ & 98597 & 31 \\
\hline 30 & $\overline{\mathbf{I}} \cdot 368 \mathrm{I} 9$ & $\overline{\mathbf{I}} \cdot 38 \circ 35$ & 0.61965 & $\overline{\mathbf{I}} .98783$ & $\overline{\mathbf{I}} \cdot 39860$ & $\overline{\mathbf{I}} \cdot 4 \mathbf{1} 266$ & 0.58734 & $\overline{\mathbf{I}} .98594$ & 30 \\
\hline $3^{1}$ & 36871 & $3^{8}$ o9 I & 61909 & 98780 & 39909 & 41318 & 58682 & 98591 & 29 \\
\hline 32 & 36924 & 38147 & 61853 & 98777 & 39958 & 41370 & 58630 & 98588 & 28 \\
\hline 33 & 36976 & 38202 & 61798 & 98774 & 40006 & 41422 & $5^{8} 578$ & 98584 & 27 \\
\hline 34 & 37028 & $3^{8} 257$ & 61743 & $9877^{1}$ & 40055 & 41474 & $5^{8} 526$ & $9858 \mathbf{I}$ & 26 \\
\hline 35 & $\overline{\mathbf{I}} \cdot 3708 \mathbf{I}$ & $\overline{1} \cdot 38313$ & 0.61687 & $\overline{\mathbf{I}} .98768$ & $\overline{\mathrm{I}} .40103$ & $\overline{\mathbf{I}} \cdot 4 \mathbf{1} 526$ & $0.5^{8} 474$ & $\overline{\mathbf{I}} .98578$ & 25 \\
\hline 36 & 37133 & 38368 & 61632 & 98765 & $4015^{2}$ & 41578 & 58422 & 98574 & 24 \\
\hline 37 & 37185 & $3^{8} 423$ & 61577 & 98762 & 40200 & 41629 & $5^{8} 371$ & 9857 I & 23 \\
\hline $3^{8}$ & 37237 & 38479 & $6152 \mathrm{I}$ & 98759 & 40249 & $4168 I$ & $5^{8} 319$ & 98568 & 22 \\
\hline 39 & 37289 & 38534 & 61466 & 98756 & 40297 & 41733 & 58267 & 98565 & 21 \\
\hline 40 & $\overline{\mathbf{I}} \cdot 3734 \mathbf{I}$ & $\overline{\mathbf{I}} \cdot 3^{8} 5^{89}$ & 0.61411 & $\overline{\mathbf{I}} .98753$ & $\bar{I} .40346$ & $\overline{\mathbf{I}} .4 \mathrm{I} 784$ & $0.5^{8} 216$ & $\overline{\mathbf{I}} .9856 \mathbf{I}$ & 20 \\
\hline 41 & 37393 & 38644 & $6135^{6}$ & 98750 & 40394 & 41836 & $5^{8} 164$ & $9855^{8}$ & 19 \\
\hline 42 & 37445 & 38699 & 61301 & 98746 & 40442 & 41887 & 58113 & 98555 & 18 \\
\hline 43 & 37497 & $3^{8} 754$ & 61246 & 98743 & 40490 & 41939 & 58061 & $9855 \mathrm{I}$ & 17 \\
\hline 44 & 37549 & 38808 & 61192 & 98740 & 40538 & 41990 & 58010 & 98548 & 16 \\
\hline 45 & $\overline{\mathrm{I}} .37600$ & $\overline{\mathbf{I}} \cdot 38863$ & 0.61137 & $\overline{\mathbf{I}} .98737$ & $\overline{\mathbf{I}} \cdot 40586$ & $\overline{\mathrm{I}} .4204 \mathrm{I}$ & 0.57959 & $\overline{\mathrm{I}} .98545$ & 15 \\
\hline 46 & 37652 & $3^{8918}$ & 61082 & 98734 & 40634 & $4^{2} 093$ & 57907 & 9854 I & 14 \\
\hline 47 & 37703 & 38972 & 61028 & $9873 \mathbf{1}$ & 40682 & 42144 & 57856 & 98538 & 13 \\
\hline 48 & 37755 & 39027 & 60973 & 98728 & 40730 & 42195 & 57805 & 98535 & 12 \\
\hline 49 & 37 & 39082 & 60918 & 98725 & 40778 & 42246 & 57754 & 98531 & II \\
\hline 50 & $\overline{\mathbf{I}} \cdot 3785^{8}$ & $\overline{\mathbf{1}} .39$ I 36 & 0.60864 & $\overline{\mathbf{I}} .98722$ & $\overline{\mathrm{I}} .40825$ & $\overline{\text { I. }} 42297$ & 0.57703 & $\overline{\mathrm{I}} .98528$ & 10 \\
\hline $5^{1}$ & 37909 & 39190 & 0810 & 98719 & 40873 & 42348 & 57652 & 98525 & 9 \\
\hline 52 & 37960 & 39245 & 60755 & 98715 & $4092 \mathrm{I}$ & 42399 & 57601 & $9852 \mathrm{I}$ & 8 \\
\hline 53 & 38 O I I & 39299 & 60701 & 98712 & 40968 & 42450 & $5755^{\circ}$ & 98518 & 7 \\
\hline 54 & 38062 & 39353 & 60647 & 98709 & 41 OI 6 & 42501 & 57499 & 98515 & 6 \\
\hline 55 & $\overline{1} \cdot 38113$ & $\overline{\mathbf{I}} \cdot 39407$ & 0.60593 & $\overline{\mathbf{I}} .98706$ & $\overline{\mathbf{I}} .41063$ & $\overline{1} \cdot 4^{2} 55^{2}$ & $0.5744^{8}$ & $\overline{\mathbf{I}} .9851 \mathrm{I}$ & 5 \\
\hline 56 & 38164 & 39461 & 60539 & 98703 & 4 I I I I & 42603 & 57397 & 98508 & 4 \\
\hline 7 & 38215 & 39515 & 60485 & 98700 & $4^{1} 1158$ & 42653 & 57347 & 98505 & 3 \\
\hline 5 & 38266 & 39569 & $6043 I$ & 98697 & 41205 & 42704 & 57296 & 98501 & 2 \\
\hline 59 & $3^{8} 317$ & 39623 & 60377 & 98694 & 41252 & 42755 & 57245 & 98498 & I \\
\hline \multirow[t]{2}{*}{60} & $\overline{\mathbf{1}} \cdot 38.368$ & $\overline{\mathbf{1}} .39677$ & 0.60323 & $\overline{\mathrm{I}} .98690$ & $\overline{\mathrm{I}} .4 \mathrm{I} 300$ & $\overline{\mathrm{I}} .42805$ & 0.57195 & $\overline{1} .9^{8} 494$ & $0^{\prime}$ \\
\hline & $\log \cos$ & $\log \cot$ & $\log \tan$ & $\log \sin$ & $\log \cos$ & $\log \cot$ & $\log \tan$ & $\log \sin$ & \\
\hline
\end{tabular}




\begin{tabular}{|c|c|c|c|c|c|c|c|c|c|}
\hline ' & $\log \sin$ & $\log \tan$ & $\log \cot$ & $\log \cos$ & $\log \sin$ & $\log \tan$ & $\log \cot$ & $\log c 0 s$ & \\
\hline $0^{\prime}$ & $\overline{1} \cdot 41 j 00$ & $\overline{1} .42 \mathrm{SO}_{5}$ & 0.57195 & 1.05 494 & $\overline{1} .+0_{34}$ & $\overline{\mathbf{1}} .45750$ & 0.54250 & $\overline{1} .99284$ & $60^{\prime}$ \\
\hline 1 & 41347 & 42856 & $571+4$ & $9^{8} 491$ & 44078 & 45797 & $542 \mathrm{O}_{3}$ & $9{ }^{S} 2 \mathrm{Si}$ & 59 \\
\hline 2 & 41394 & 42906 & 57094 & 98488 & 44122 & $458+5$ & $54 \times 55$ & $9 \$ 277$ & $5^{8}$ \\
\hline 3 & +11441 & 42957 & 57043 & $98+84$ & 44166 & 45892 & 54108 & 98273 & 57 \\
\hline 4 & $4^{\prime} 4^{83}$ & 43007 & 56993 & 98481 & 44210 & 45940 & 54060 & $9 S 270$ & $5^{6}$ \\
\hline 5 & $\overline{\mathrm{I}} .41535$ & $\overline{1} .43057$ & 0.56943 & $\overline{1} .98477$ & $\overline{1} .44253$ & $\overline{\mathbf{I}} .45987$ & 0.54013 & $\overline{1} .9 \$ 266$ & 55 \\
\hline 6 & 41552 & 43108 & $56 \$ 92$ & 98474 & +4297 & 46035 & 53965 & 98262 & 54 \\
\hline 7 & 41628 & 43158 & 56842 & $98+71$ & $4434 \mathrm{I}$ & 46082 & 53918 & 98259 & 53 \\
\hline 8 & 41675 & 43208 & 56792 & 98467 & +4385 & 46 I30 & 53870 & 98255 & $5^{2}$ \\
\hline 9 & 41722 & 432 & $5^{6}$ & 98464 & $44+28$ & 46 & 3 & 51 & $5^{1}$ \\
\hline 10 & $\overline{1} \cdot 41 ; 6 \mathrm{~S}$ & $\overline{\mathbf{I}} .43308$ & $0.5^{6} 692$ & I. $9 \$ 460$ & $\overline{\mathrm{I}} \cdot 44+72$ & $\bar{I} \cdot 46224$ & 0.53776 & $\overline{\mathbf{I}} .98248$ & 50 \\
\hline 11 & $41 \$ 15$ & $4335^{\mathrm{s}}$ & 56642 & 98457 & 44516 & 46271 & 53729 & 95244 & 49 \\
\hline 12 & 41861 & 43408 & 56592 & $9^{S} 453$ & 44559 & 46319 & 53681 & 98240 & $4^{8}$ \\
\hline 13 & $4^{1} \mathrm{gos}$ & $4345^{8}$ & 56542 & 95450 & 44602 & 46366 & 53634 & 98237 & 47 \\
\hline 14 & 41954 & 43508 & 56492 & $9^{8}+47$ & 44646 & $46+13$ & $535^{87}$ & 98233 & 46 \\
\hline 15 & $\overline{1} .42001$ & $\overline{1} .4355^{8}$ & $0.56+4^{2}$ & '. .98443 & T. 44689 & $\overline{1} .46460$ & 0.53540 & $\overline{1} .98229$ & 45 \\
\hline 16 & 42047 & 43607 & 56393 & 98440 & 44733 & 46507 & 53493 & 98226 & 44 \\
\hline 17 & 42093 & 43657 & $5^{6} 343$ & $9^{8}+3^{6}$ & 44776 & 46554 & $5344^{6}$ & 98222 & 43 \\
\hline 18 & 42140 & 43707 & 56293 & 99433 & 44819 & 46601 & & 98218 & $4^{2}$ \\
\hline 19 & 42156 & $4375^{6}$ & 56244 & 98429 & 44862 & 46648 & $5335^{2}$ & $9 S 215$ & 41 \\
\hline 20 & $\overline{1} .42232$ & $\overline{\mathbf{I}} .43806$ & 0.56194 & $\overline{1} .98426$ & $\overline{\mathrm{I}} .44905$ & $\overline{\mathbf{I}} .46694$ & 0.53306 & $\overline{\mathrm{I}} .9821 \mathrm{I}$ & 40 \\
\hline 21 & +2278 & $43 S_{55}$ & 56145 & 98422 & 44948 & 46741 & 53259 & $9 S 207$ & 39 \\
\hline 22 & 42324 & 43905 & 56095 & 98419 & 44992 & 46788 & 53212 & 98204 & $3^{8}$ \\
\hline 23 & $4237^{\circ}$ & 43954 & 56046 & 98415 & $45 \bigcirc 35$ & 46835 & 53165 & $9^{S} 200$ & 37 \\
\hline 24 & 42416 & 44004 & 55996 & 98412 & 45077 & 46881 & 53119 & 98196 & 36 \\
\hline 25 & $\overline{\mathrm{I}} \cdot 42+46 \mathrm{I}$ & $\overline{1} \cdot 44053$ & 0.55947 & $\overline{\mathbf{I}} .98409$ & $\overline{1} .45120$ & $\overline{\mathbf{I}} .46928$ & 0.53072 & $\overline{1} .99192$ & 35 \\
\hline 26 & 42507 & 44102 & 55898 & 98405 & 45163 & 46975 & 53025 & 98189 & 34 \\
\hline 27 & 42553 & $44^{1} 5^{1}$ & 55849 & $9^{8} 402$ & 45206 & 47021 & 52979 & 98185 & 33 \\
\hline 28 & 42599 & 44201 & 55799 & 98398 & $45 \quad 2.49$ & 47068 & 52932 & 98181 & 32 \\
\hline 29 & 42644 & 44250 & $5575^{\circ}$ & 98395 & 45292 & 47114 & 52886 & 98177 & 31 \\
\hline 30 & $\overline{1} .42600$ & $\overline{\mathrm{I}} \cdot 4 \mathbf{4 9 9}$ & 0.55701 & I. 98391 & $\overline{\mathbf{I}} .45334$ & $\overline{\mathrm{I}} .47160$ & 0.528 .40 & $\overline{\mathbf{I}} .98174$ & 30 \\
\hline $3^{i}$ & 42735 & 44348 & 55652 & 98388 & 45377 & 47207 & $5^{2} 793$ & 98170 & 29 \\
\hline 32 & 42751 & 44397 & 55603 & $9^{8} 3^{8} 4$ & 45419 & 47253 & 52747 & 98166 & 28 \\
\hline 33 & 42826 & $4444^{6}$ & 55554 & 98381 & 45462 & 47299 & $5^{2} 701$ & 98162 & 27 \\
\hline 34 & $42 \$_{72}$ & 44495 & 55505 & 98377 & 45504 & 47346 & 52654 & 98159 & 26 \\
\hline 35 & 7.42917 & I. 44544 & 0.55456 & $\overline{1} .98373$ & $\overline{\mathrm{I}} .45547$ & $\overline{\mathrm{I}} .47392$ & 0.52608 & $\overline{\mathbf{I}} .9 \mathrm{~S} 155$ & 25 \\
\hline 36 & 42962 & 44592 & 55408 & $9^{5} 370$ & 45559 & $4743^{S}$ & $5^{2} 5^{62}$ & 98151 & 24 \\
\hline 37. & $43 \mathrm{vos}$ & 44641 & 55.359 & $9^{8} 3^{66}$ & 45632 & $47+84$ & 52516 & $9 S 1+7$ & 23 \\
\hline $3^{5}$ & $43 \circ 53$ & 44690 & 55310 & 98363 & 45674 & 47530 & 52470 & 98144 & 22 \\
\hline 39 & 43 ors & 44738 & 55262 & 98359 & 45716 & $4757^{6}$ & 52424 & 98140 & $2 \mathrm{I}$ \\
\hline 40 & I. 43143 & $\overline{\mathrm{I}} \cdot 447^{87}$ & 0.55213 & $\overline{1} .98356$ & $\overline{\mathrm{I}} .4575^{8}$ & $\overline{\mathrm{I}} .47622$ & $0.5^{2} 37^{8}$ & $\overline{1} .99136$ & 20 \\
\hline 41 & 43158 & 44836 & $55 \quad 164$ & 08352 & $45 \mathrm{SOI}$ & 47665 & 52332 & $9 S 132$ & 19 \\
\hline 42 & 43233 & $44 \mathrm{SS}_{4}$ & 55116 & $9^{8} 349$ & $45 S_{43}$ & 47714 & $5^{2} 286$ & $9 \$ 129$ & 18 \\
\hline 43 & 4.3278 & 44933 & 55067 & $9^{8} 345$ & $4588_{5}$ & 47760 & $5^{2} 240$ & $9 \$ 125$ & 17 \\
\hline 44 & 43323 & 44951 & 55019 & 98342 & 45927 & $47 \mathrm{So6}$ & 52194 & 98121 & 16 \\
\hline 45 & $\overline{1} .43 .367$ & T.45029 & 0.54971 & $\overline{1} .9^{\mathrm{S}} 33^{\mathrm{S}}$ & $\overline{1} .45969$ & $\overline{1} .47 \delta_{52}$ & 0.52148 & $\overline{1} .98117$ & 15 \\
\hline 46 & 43412 & 45075 & 54922 & 98334 & 46011 & 47897 & 52103 & 95113 & 14 \\
\hline 47 & 4.3457 & 45126 & 54874 & $9^{8} 331$ & 46053 & 47943 & 52057 & 98 & 13 \\
\hline 45 & 43502 & 45174 & 54826 & 98327 & 46 ons & 47959 & 52011 & 95106 & 12 \\
\hline 49) & $4334^{6}$ & 45222 & 54778 & 98324 & 46136 & 48035 & 51965 & 98102 & 11 \\
\hline 50 & T. 43591 & T.45 271 & 0.54729 & T. 98 S 320 & $1.4617^{8}$ & T. 48 oso & 0.51920 & T.os ogs & 10 \\
\hline 51 & 436,35 & 45,319 & 54681 & 95317 & 46220 & 45126 & 51574 & 98094 & \\
\hline $5^{2}$ & 43650 & 45367 & 54633 & is 313 & $4^{62} 262$ & 48171 & 51829 & $9^{5}$ ono & 8 \\
\hline 53 & 43724 & 45415 & $545^{55}$ & 95304 & $4^{6} 303$ & 48217 & 51753 & 95057 & 7 \\
\hline 54 & $437^{6}(0)$ & $454^{6} 3$ & 54537 & 98306 & $4^{\text {t) } 345}$ & 48262 & $5173^{5}$ & $0 \mathrm{SOS}_{3}$ & 6 \\
\hline 55 & 1.43813 & I.45511 & 0.544 .59 & T. 99302 & $7.4^{6} 3.56$ & T.4. 307 & 0.51693 & 7.98079 & 5 \\
\hline 56 & $43 \times 57$ & 45554 & 54441 & ys 300 & 46428 & 45353 & 51647 & 98075 & 4 \\
\hline 57 & 43001 & $45(x \times 6)$ & 54394 & 98205 & 46) $4(x)$ & 45395 & 51602 & $9809 i$ & : \\
\hline $5^{s}$ & 43946 & 45654 & 54346 & $9^{5} \mathrm{zmi}$ & $4^{6} 511$ & 48443 & 51557 & is oby & 2 \\
\hline $5 ?$ & $439 \%$ & 45702 & $5420 \mathrm{~S}$ & 98258 & 46552 & $45_{4}$ & $515 i i$ & 95063 & 1 \\
\hline \multirow[t]{2}{*}{$60^{\prime}$} & 1.440 .34 & 1.45750 & 0.34250 & 10.825 .4 & 1.46534 & T.45 53 & 0.51466 & Ins olno & $0^{\prime}$ \\
\hline & $\log 508$ & log cot & Los tan & $\log \sin$ & $\log \mathrm{ens}$ & log bot & log tan & $\log \sin$ & \\
\hline
\end{tabular}




\begin{tabular}{|c|c|c|c|c|c|c|c|c|c|}
\hline ! & $\log \sin$ & $\log \tan$ & $\log \cot$ & $\log 008$ & $\log \sin$ & $\log \tan$ & $\log \cot$ & $\log c 08$ & \\
\hline $0^{\prime}$ & i. 40594 & 1.45534 & 0.51406 & $\overline{1} .98060$ & $\overline{1} .48998$ & $\overline{1} .51178$ & $0.48 \$ 22$ & $\overline{\mathrm{I}} .9782 \mathrm{I}$ & $60^{\prime}$ \\
\hline I & 46635 & $4^{\$ 579}$ & $5 \mathrm{I}+2 \mathrm{I}$ & 98056 & 49037 & 51221 & $4^{8} 779$ & 97817 & 59 \\
\hline 2 & 46676 & 48624 & 51376 & 98052 & $49 ? 76$ & 51264 & 48736 & 97812 & 58 \\
\hline 3 & $467^{17}$ & 48669 & 51331 & 98048 & 49 II 5 & 51306 & 48694 & 97808 & 57 \\
\hline 4 & 46758 & $4^{5} 714$ & 51236 & $9 S 0+4$ & 49153 & 51349 & 48651 & 97804 & 56 \\
\hline 5 & $\overline{1} .46 S 00$ & $\overline{1} .4^{\$} 759$ & 0.51241 & $\overline{\mathbf{I}} .980 .40$ & $\overline{\mathrm{I}} .49192$ & $\overline{\mathbf{I}} \cdot 51392$ & 0.48608 & $\overline{\mathbf{I}} .97800$ & 55 \\
\hline 6 & $46 S_{41}$ & $4 \mathrm{SO}_{4}$ & 51196 & 98036 & 49231 & 51435 & 48565 & 97796 & 54 \\
\hline 7 & $46 \$ \$ 2$ & 48849 & 51151 & 98032 & 49269 & 51478 & 48522 & & 53 \\
\hline 8 & 46923 & 48994 & 51106 & 98029 & 49308 & $5^{1} 520$ & 48480 & 97788 & 52 \\
\hline 9 & 46 & $4^{8}$ & 51 & 98 & 49347 & & 48437 & 97784 & 51 \\
\hline 10 & $\bar{I} .47005$ & $\overline{\mathrm{I}} \cdot 4898_{4}$ & 0.51016 & $\bar{I} .98021$ & $\overline{\mathbf{I}} .493^{85}$ & $\overline{\mathbf{1}} \cdot 51606$ & 0.48394 & $\overline{\mathbf{I}} .97779$ & 50 \\
\hline II & 47045 & 49029 & 50971 & 98017 & 49424 & 51648 & 48352 & 97775 & 49 \\
\hline 12 & 47086 & 49073 & 50927 & 98013 & 49462 & 51691 & $4^{8} 309$ & $9777^{1}$ & $4^{8}$ \\
\hline 13 & 47127 & 49 II 8 & 50882 & 98009 & 49500 & 51734 & 48266 & 97767 & 47 \\
\hline 14 & 47168 & $49 \mathrm{I}_{3}$ & 50837 & 98005 & 49539 & 51776 & 48224 & 97763 & 46 \\
\hline 15 & $\overline{\mathrm{I}} .47209$ & $\overline{\mathrm{I}} .49207$ & 0.50793 & $\overline{\mathbf{I}} .9800 \mathrm{I}$ & $\overline{\mathrm{I}} .49577$ & $\overline{\mathbf{I}} .51819$ & $0.4818 \mathrm{I}$ & $\overline{\mathbf{1}} .97759$ & 45 \\
\hline 16 & 47249 & 49252 & 50748 & 97997 & 49615 & 5 I 86 I & 48139 & 97754 & 44 \\
\hline 17 & 47290 & 49 & 50704 & & 49654 & $5^{1} 903$ & 48097 & & 43 \\
\hline 18 & 47330 & $4934 \mathrm{I}$ & 50659 & 97989 & 49692 & 51946 & 48054 & 97746 & $4^{2}$ \\
\hline 19 & 47 & 49 & 50615 & 97 & 49730 & $5^{1}$ & 48 o1 2 & 97 & 41 \\
\hline 20 & $\overline{\mathrm{I}} .4741 \mathrm{I}$ & $\overline{1} .49430$ & 0.50570 & $\overline{\mathbf{I}} .97982$ & $\overline{\mathbf{I}} .49768$ & $\overline{\mathrm{I}} \cdot 52031$ & 0.47969 & $\overline{\mathbf{I}} \cdot 9773^{8}$ & 40 \\
\hline 21 & $4745^{2}$ & 49 & 50526 & 97978 & 49806 & 73 & 47927 & 97 & 39 \\
\hline 22 & 47492 & & $5048 I$ & & 49844 & & 47885 & & 38 \\
\hline 23 & 47533 & 49563 & 50437 & 97970 & 49882 & 52157 & 47843 & 97 & 37 \\
\hline 24 & 47573 & 49607 & 50393 & 97966 & 49920 & $5^{2}$ & 47800 & 97 & 36 \\
\hline 25 & $\overline{1} .47613$ & $\overline{\mathbf{I}} .49652$ & 0.50348 & $\overline{\mathbf{I}} .97962$ & $\overline{\mathrm{I}} \cdot 4995^{8}$ & $\overline{\mathbf{I}} \cdot 52242$ & 0.47758 & $\overline{\mathbf{I}} .97$ & 35 \\
\hline 26 & 47654 & 49696 & 50304 & $9795^{8}$ & 49996 & 52284 & 47716 & & 34 \\
\hline 27 & 47694 & $4974^{\circ}$ & 50260 & 97954 & 50034 & 26 & 47674 & & 33 \\
\hline 28 & 47734 & 49784 & 50216 & 97 & 50072 & 68 & 47632 & 97 & $3^{2}$ \\
\hline 29 & 47774 & 49828 & 50172 & 97946 & 50110 & 52410 & 47590 & 97700 & 31 \\
\hline 30 & $\overline{\mathrm{I}} \cdot 47814$ & $\overline{\mathbf{I}} .49872$ & 0.50128 & $\overline{\mathbf{1}} .97$ & $\overline{\mathbf{1}} .50148$ & $\overline{\mathbf{x}} \cdot 5^{2}$ & 0.47548 & $\overline{\mathbf{x}} .97$ & 30 \\
\hline $3 \mathrm{I}$ & & & 50084 & & 50185 & & 47506 & $9769 \mathrm{I}$ & 29 \\
\hline $3^{2}$ & 47894 & 49960 & 50040 & 97 & 50223 & 36 & 47464 & 97687 & 28 \\
\hline 33 & 47934 & 50004 & 49996 & & 50261 & $5^{2} 578$ & 47422 & 97683 & 27 \\
\hline 34 & 47974 & $\cdot 50048$ & 49952 & 26 & 50298 & 52620 & 47380 & 97679 & 26 \\
\hline 35 & $\overline{\mathrm{I}} .480 \mathrm{OI} 4$ & $\overline{\mathrm{I}} \cdot 50092$ & 0.49908 & $\overline{\mathbf{I}} .97922$ & $\overline{\mathbf{I}} .50336$ & $\overline{\mathbf{I}} .5266 \mathrm{I}$ & 0.47339 & $\overline{\mathbf{I}} .97674$ & 25 \\
\hline $3^{6}$ & 48054 & 50136 & 49864 & & 50374 & $5^{2} 703$ & 47297 & & 24 \\
\hline 37 & 48094 & 50 I 80 & 49820 & & 504 I I & $5^{2}$ & 47255 & 97666 & 23 \\
\hline $3^{8}$ & $48 \div 33$ & 50223 & 49777 & 0 & 50449 & 52 & 47213 & 97662 & 22 \\
\hline 39 & 48173 & 50267 & 49733 & 97906 & 50486 & 52829 & 47 I I & 97657 & 21 \\
\hline 40 & $\overline{\mathrm{I}} \cdot 48213$ & $\overline{\mathrm{I}} \cdot 503 \mathrm{II}$ & 0.49689 & $\overline{\mathbf{I}} .97$ & $\overline{\mathbf{x}} .50523$ & $\overline{\mathbf{I}} \cdot 52870$ & 0.47130 & $\overline{\mathbf{I}} .9$ & 20 \\
\hline 41 & 48252 & 50 355 & 49645 & 97 & $5056 r$ & 52912 & 47088 & 97 & 19 \\
\hline 42 & 18 & & 49 & & 98 & & 47047 & & 18 \\
\hline 43 & 48332 & 50442 & $4955^{8}$ & & 50635 & 95 & 47005 & & 17 \\
\hline 44 & $4837 \mathrm{I}$ & 50485 & $495^{15}$ & & 50673 & $53 \circ 37$ & 46963 & 97636 & 16 \\
\hline 45 & $\overline{\mathrm{I}} .484 \mathrm{I} \mathrm{I}$ & $\bar{I} \cdot 50529$ & $0.4947 \mathrm{I}$ & $\overline{\mathrm{I}} .97882$ & $\overline{\mathrm{I}} .50710$ & $\overline{\mathbf{I}} \cdot 53078$ & 0.46922 & $\overline{\mathbf{I}} .97632$ & 15 \\
\hline $4^{6}$ & & & 28 & & 50 & & 46880 & 8 & 14 \\
\hline 47 & & 16 & 49384 & & & & 46839 & 23 & 13 \\
\hline $4^{8}$ & & 50659 & $4934 \mathrm{I}$ & & & & & 19 & 12 \\
\hline 49 & & 50703 & 49297 & & $5^{8}$ & 44 & $4^{6} 75^{6}$ & 15 & II \\
\hline 50 & $\overline{1} .48607$ & $\overline{\mathrm{I}} \cdot 50746$ & 0.49254 & $\overline{\mathbf{I}} .9786 \mathrm{I}$ & $\overline{\mathbf{I}} \cdot 50896$ & $\overline{\mathbf{I}} \cdot 53285$ & 0.46715 & $\overline{\mathbf{I}} .97610$ & 10 \\
\hline $5^{1}$ & & & $492 \mathrm{II}$ & & 50933 & & & & 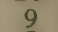 \\
\hline $5^{2}$ & & 33 & 49 & & & & 32 & $\mathrm{O} 2$ & 8 \\
\hline 53 & 25 & 50876 & 49 & & 51007 & 53409 & $4^{6} 59 \mathrm{I}$ & 97597 & 7 \\
\hline 54 & & & $4908 \mathrm{I}$ & & 51043 & $5345^{\circ}$ & 46550 & 97593 & 6 \\
\hline 55 & $\overline{\mathrm{I}} .488 \mathrm{O}_{3}$ & $\overline{\mathrm{I}} .50962$ & 0.49038 & $\overline{\mathbf{I}} .9784 \mathrm{I}$ & $\overline{\mathbf{I}} \cdot 51080$ & $\overline{\mathbf{I}} \cdot 53492$ & 0.46508 & $\overline{\mathbf{I}} .97589$ & 5 \\
\hline 56 & 48842 & 51005 & 48995 & 97837 & 51117 & 53533 & 46467 & 97584 & 4 \\
\hline 57 & $4888 \mathbf{I}$ & $5^{10} 048$ & 48952 & 97833 & 51154 & 53574 & 46426 & 97580 & 3 \\
\hline $5^{8}$ & $48 ? 20$ & $5^{1092}$ & $48 c$ & & $5119 I$ & & $4^{6} 3^{8} 5$ & 97576 & 2 \\
\hline & 48959 & 51135 & 48865 & 97825 & 51227 & 53656 & 46344 & 97571 & I \\
\hline \multirow[t]{2}{*}{$60^{\prime}$} & $\overline{\mathrm{I}} .48998$ & $\overline{\mathrm{I}} .51178$ & 0.48822 & $\overline{1} .97821$ & $\overline{\mathrm{I}} .5 \mathrm{I} 264$ & $\overline{1} .53697$ & 0.46303 & $\overline{\mathrm{I}} .97567$ & $0^{\prime}$ \\
\hline & $\log \cos$ & $\log \cot$ & $\log \tan$ & $\log \sin$ & $\log \cos$ & $\log \cot$ & $\log \tan$ & $\log \sin$ & 1 \\
\hline
\end{tabular}




\begin{tabular}{|c|c|c|c|c|c|c|c|c|c|}
\hline ? & $\log \sin$ & $\log \tan$ & $\log \cot$ & $\log \cos$ & $\log \sin$ & $\log \tan$ & $\log \cot$ & $\log \cos$ & \\
\hline $0^{\prime}$ & $\overline{\mathrm{I}} \cdot 51264$ & $\overline{1} .53097$ & 0.46303 & 1.97507 & 1.53405 & $\begin{array}{ll}1.56 & 107\end{array}$ & 0.43893 & 1.97299 & $60^{\prime}$ \\
\hline I & 51301 & $5373^{8}$ & 46262 & 97563 & 53440 & $561+6$ & $43 S_{54}$ & 97294 & 59 \\
\hline 2 & $5^{1} 33^{S}$ & 53779 & 46221 & $9755^{\circ}$ & 53475 & $5615_{5}$ & 43 SI 5 & 97259 & $5^{s}$ \\
\hline 3 & 51374 & 53820 & 46180 & 97554 & 53509 & $5^{6} \div 224$ & $4377^{6}$ & 97285 & 57 \\
\hline 4 & $5^{1} 411$ & 53861 & 46139 & 97550 & 53544 & 56264 & 43736 & 97250 & 56 \\
\hline 5 & '. 51447 & '. 53902 & 0.46098 & '̄.97545 & '. $5357^{8}$ & $\overline{\mathbf{1}} .56303$ & 0.43697 & $\overline{\mathbf{I}} .97276$ & 55 \\
\hline 6 & $514^{8} 4$ & 53943 & 46057 & 97541 & 53613 & 56342 & $4365^{8}$ & 97271 & 54 \\
\hline 7 & 51520 & $5399_{4}$ & 46016 & 97536 & 53647 & $5^{6} 3^{81}$ & 43619 & 97266 & 53 \\
\hline 8 & $5^{1} 557$ & 54025 & 45975 & 97532 & 53682 & 56420 & 43550 & 97262 & $5^{2}$ \\
\hline 9 & $5^{1} 593$ & 54065 & 35 & 97528 & 53716 & 56459 & 43 & 97 & $5^{1}$ \\
\hline 10 & 1.51 629 & I. 54106 & 0.45894 & 1.97523 & I. $5375^{1}$ & 1.56498 & 0.43502 & I. 97 & 50 \\
\hline 11 & 51666 & 54147 & 45853 & 97519 & $537^{85}$ & 56537 & 43463 & 97248 & 49 \\
\hline 12 & 51702 & 54187 & $45^{813}$ & 97515 & 53819 & 56576 & 43424 & 97243 & 48 \\
\hline 13 & 51738 & 54228 & 45772 & $975^{10}$ & 53854 & 56615 & 43.385 & $9723^{8}$ & 47 \\
\hline 14 & 51774 & 54 & 45731 & 26 & 53 & 56654 & 46 & 34 & 46 \\
\hline 15 & I. 51 SII & I. 54309 & 0.45691 & I. 97501 & I. 53922 & I. 56693 & 0.43307 & I. 97229 & 45 \\
\hline 16 & 51847 & $5+35^{\circ}$ & 45650 & 97497 & 53957 & 56732 & 43268 & 97224 & 44 \\
\hline 17 & $5188_{3}$ & 54390 & 45610 & 97492 & 5399 I & $5677^{1}$ & 43229 & 97220 & 43 \\
\hline 18 & 51919 & $5443^{1}$ & 45569 & 97455 & 54 & 56810 & 0 & 5 & 42 \\
\hline 19 & $5^{1} 955$ & 54 & 9 & 97484 & 9 & 56849 & $5^{I}$ & 10 & 41 \\
\hline 20 & 1. 51 & 1. 54 & 0.45 & 1.9 & I. 5 & I. 56 & 0.4 & I. 9 & 40 \\
\hline $\begin{array}{l}21 \\
22\end{array}$ & $\begin{array}{l}52027 \\
52063\end{array}$ & 54 & $45+48$ & 75 & 7 & 56 & 4 & 1 & . 39 \\
\hline 23 & 52099 & $\begin{array}{l}54593 \\
54633\end{array}$ & $\begin{array}{ll}45 & 407 \\
45 & 367\end{array}$ & 97466 & & $\begin{array}{l}50905 \\
57004\end{array}$ & 35 & & 38 \\
\hline 24 & 52135 & 54673 & 45327 & 97461 & 54229 & 57042 & 42958 & 97187 & 36 \\
\hline 25 & I. 52171 & $\overline{\mathrm{I}} \cdot 54714$ & 0.45286 & $\overline{\mathrm{I}} .97457$ & $\overline{\mathrm{I}} \cdot 54263$ & $\overline{\mathrm{I}} \cdot 57 \mathrm{OSI}$ & 0.42919 & $\overline{1} .97 \mathrm{IS2}$ & 35 \\
\hline 26 & 52207 & 54754 & 45246 & 97453 & 54297 & 57120 & $42 S S 0$ & 97178 & 34 \\
\hline 27 & 52242 & 54794 & 45206 & $9744^{8}$ & 54331 & $5715^{8}$ & 42842 & 97173 & 33 \\
\hline 28 & $5227^{8}$ & 54835 & 45165 & 97444 & 65 & 57197 & $42 \mathrm{SO}_{3}$ & 97168 & $3^{2}$ \\
\hline 29 & $5^{2} 314$ & 54875 & 45125 & 97439 & 54399 & 57235 & 42765 & 97163 & 31 \\
\hline 30 & $\overline{\mathrm{I}} .5^{2} 35^{\circ}$ & $\bar{I} \cdot 54915$ & 0.45085 & $\overline{\mathbf{I}} .97435$ & $\overline{\mathbf{I}} \cdot 54433$ & $\overline{\mathbf{I}} .57274$ & $0.4^{2} 726$ & $\overline{\mathbf{I}} .97159$ & 30 \\
\hline 31 & $5^{2} 3^{85}$ & 54955 & 45045 & 30 & 54466 & 57312 & 42688 & 97154 & 29 \\
\hline 32 & $5^{2421}$ & 4995 & 45005 & 97426 & 54 & 57351 & 42649 & 97149 & 28 \\
\hline 33 & $5^{2} 456$ & $55 \circ 35$ & 44965 & 97421 & 54534 & 57389 & 42611 & 97145 & 27 \\
\hline 34 & $5^{2} 49^{2}$ & 55075 & 44925 & 97417 & 54567 & 57428 & $4257^{2}$ & 97140 & 26 \\
\hline 35 & $\overline{\mathrm{i}} .52527$ & 1. 55115 & $0.4483_{5}$ & 1. 97412 & i. 54601 & I. 57466 & 0.42534 & '. 97 I 35 & 25 \\
\hline 36 & 52563 & 55155 & 44845 & 97408 & 54635 & 57504 & $4^{2} 49^{6}$ & 97130 & 24 \\
\hline 37 & $5^{2} 598$ & 55195 & 44805 & 97403 & 54608 & 57543 & $4^{2} 457$ & 97126 & 23 \\
\hline $3^{8}$ & $5^{26} 634$ & 55235 & 44765 & 97399 & 54702 & 57581 & 42419 & 97121 & 22 \\
\hline 39 & 52669 & 55275 & 44725 & 97394 & 54735 & 57619 & $4^{2} 3^{81}$ & 97116 & 21 \\
\hline 40 & $\bar{I} .52705$ & $\overline{1} .55315$ & 0.44685 & $\overline{\mathbf{I}} .97390$ & $\overline{\mathbf{I}} .54760$ & '. 57658 & 0.42342 & $\overline{1.97111}$ & 20 \\
\hline $4 !$ & $5^{2} 740$ & 55355 & 44645 & 97355 & $54 \mathrm{SO} 2$ & 57696 & $4^{2} 304$ & 97107 & 19 \\
\hline 42 & $5^{2} 775$ & 55395 & 44605 & 97381 & $54: 36$ & 57734 & 42206 & 97102 & 18 \\
\hline 43 & $5^{2} 811$ & 554.34 & 44566 & $9737^{6}$ & 54869 & 57772 & 42228 & 97097 & 17 \\
\hline 44 & 52846 & 55474 & 44526 & 97372 & 54903 & 57 S10 & 42190 & 97092 & 16 \\
\hline 45 & $\overline{1} .52881$ & $\overline{1} .55514$ & $0.444^{56}$ & I.97367 & '. 54936 & $\overline{1} .57 \$_{49}$ & 0.42151 & i. 97087 & 15 \\
\hline 46 & 52916 & 55554 & 44446 & 97363 & 54969 & 57857 & 42113 & $9708_{3}$ & 14 \\
\hline 47 & 52951 & 55593 & 44407 & $9735^{8}$ & $55 \mathrm{OO}_{3}$ & 57925 & 42075 & $9707^{8}$ & 13 \\
\hline $4^{8}$ & 52986 & 55633 & 44367 & 97353 & 55036 & 57963 & 42037 & 97073 & 12 \\
\hline 49 & 53021 & 55673 & 44327 & 97349 & $550(x)$ & 01 & 41699 & 65 & 11 \\
\hline 50 & I. 53056 & $\overline{1} .55712$ & 0.44288 & I. 97344 & I. $55 \quad 102$ & i. $5^{\circ} 0311$ & 0.41961 & 1.9700 .3 & 10 \\
\hline 51 & $530 y 2$ & 55752 & 442.48 & 97340 & 55136 & 58077 & 41023 & 97059 & ? \\
\hline 52 & 53126 & 55791 & 44209 & 97.335 & $5516 \%$ & 55115 & $418_{5}$ & 97054 & 8 \\
\hline 53 & 53161 & 55831 & $44 I(x)$ & 97331 & 55202 & $58 \quad 153$ & 41847 & 97049 & 7 \\
\hline 54 & 53196 & 55870 & 44130 & 97326 & 55235 & 55191 & 41809 & 97044 & 6 \\
\hline 55 & 1.53231 & 1.55910 & $0.44(x) 0$ & 1.97322 & 1.55268 & T. $5^{8}=29$ & 0.41771 & 1.970 .39 & 5 \\
\hline $5^{6)}$ & 53266 & 55949 & 44051 & 97.317 & 55.301 & 58267 & 41733 & 97035 & 4 \\
\hline 57 & 53301 & $55 \mathrm{ysi}$ & 44011 & $973^{12}$ & 5533.4 & 58304 & 41696 & 97030 & 3 \\
\hline $5^{8}$ & 533.6 & 51025 & 43972 & 97.305 & 55367 & 58,342 & 4165. & 97025 & 2 \\
\hline \multirow{3}{*}{60} & 53.370 & 56067 & 43933 & 97303 & 55460 & $58 ; .0$ & 41620 & 97020 & I. \\
\hline & 1.53405 & 1.56107 & 0.43503 & 1.07200 & 1.55433 & 1.55418 & 0.41552 & 1.97015 & $0^{\prime}$ \\
\hline & $\log 608$ & $\log \operatorname{ent}$ & $\log \tan$ & log $\sin$ & $\log 6 \operatorname{ses}$ & $\log e n t$ & $\log \tan$ & $\log \sin$ & \\
\hline
\end{tabular}




\begin{tabular}{|c|c|c|c|c|c|c|c|c|c|}
\hline 1 & $\log \sin$ & $\log \tan$ & $\log \cot$ & $\log \cos$ & $\log \sin$ & $\log \tan$ & $\log \cot$ & $\log \cos$ & \\
\hline $0^{\prime}$ & $\overline{\mathrm{I}} .55433$ & $\overline{1} \cdot 5^{8}+18$ & $0.4^{1} 5^{\mathrm{S} 2}$ & $\overline{\mathbf{I}} .97015$ & $\overline{1} .57 .358$ & $\overline{\mathbf{1}} .60641$ & 0.39359 & $\overline{1} .96717$ & $60^{\prime}$ \\
\hline I & 55466 & $5^{8}+55$ & 41545 & 97010 & $573^{89}$ & 60677 & 39323 & & 59 \\
\hline 2 & 55499 & $58+93$ & 41507 & 97005 & 57420 & 60714 & 39286 & 96706 & $5^{8}$ \\
\hline 3 & 55532 & $5^{S} 531$ & $414^{69}$ & 97001 & 57451 & 60750 & 39250 & 96701 & 57 \\
\hline 4 & 55564 & $5^{8} 569$ & 41431 & 968 & 57482 & 60786 & 39214 & 96 & $5^{6}$ \\
\hline 5 & I. 55597 & $\overline{\mathrm{I}} \cdot 5^{8} 606$ & 0.41394 & $\overline{1} .96991$ & $\overline{1} .57514$ & $\overline{1} .60823$ & 0.39177 & $\overline{1} .96$ & 55 \\
\hline 7 & 55630 & $5^{8644}$ & 41356 & & 57545 & $S_{59}$ & 39 & & 54 \\
\hline 8 & $\begin{array}{l}55063 \\
55695\end{array}$ & $5 s$ & $\begin{array}{lll}41 & 3 & 1 \\
41 & 29 \\
41 & 28\end{array}$ & 96 & & $\begin{array}{l}60895 \\
60931\end{array}$ & $\begin{array}{l}39 \\
39\end{array}$ & $\begin{array}{l}96681 \\
96676\end{array}$ & $\begin{array}{l}53 \\
52\end{array}$ \\
\hline 9 & 55728 & 58757 & $\begin{array}{lll}41 & 201 \\
4 & 243\end{array}$ & 96971 & 57638 & 60967 & 39033 & 96670 & $5^{1}$ \\
\hline 10 & $\overline{\mathrm{I}} .557^{6 \mathrm{I}}$ & $\overline{\mathbf{I}} \cdot 5^{8} 794$ & 0.41206 & $\overline{\mathbf{I}} .96966$ & $\overline{\mathbf{I}} \cdot 57669$ & $\overline{1} .61004$ & 0.38996 & $\overline{\mathbf{I}} .96665$ & 50 \\
\hline II & & $588_{32}$ & $\begin{array}{lll}41 & 168\end{array}$ & & & 40 & $3^{8960}$ & & 49 \\
\hline 12 & $55 \mathrm{~S} 26$ & 58869 & $\begin{array}{lll}4 & 1 & 3\end{array}$ & 57 & 31 & 61 & $3^{8}$ & 55 & 48 \\
\hline 13 & $5585^{8}$ & 58907 & 41093 & $96 \mathrm{c}$ & & 61112 & 38858 & 966 & 47 \\
\hline 14 & 55 S9I & $5^{89} 944$ & $4105^{6}$ & 96947 & 57793 & 61148 & $3^{8} \&_{52}$ & 96645 & 46 \\
\hline 15 & $\overline{\mathrm{I}} \cdot 55923$ & $\overline{\mathbf{I}} \cdot 5^{8} 98 \mathbf{I}$ & 0.41019 & $\overline{\mathbf{1}} .96942$ & $\overline{\mathbf{I}} \cdot 57824$ & $\overline{1} .61184$ & $0.3^{8816}$ & $\overline{\mathbf{I}} .96640$ & 45 \\
\hline 16 & 55956 & 59019 & $4098 \mathrm{I}$ & 96937 & 57855 & 61220 & $3^{8} 780$ & 34 & 44 \\
\hline 17 & 55988 & 59056 & 40944 & 32 & 385 & $6 I$ & 44 & 29 & 43 \\
\hline 18 & 6021 & 59094 & 40906 & & & $6 I$ & & & 42 \\
\hline 19 & 56053 & 59 I 31 & 40 & 2 & 47 & 28 & & & $4 I$ \\
\hline 20 & $\overline{\mathbf{1}} .56085$ & $\overline{\mathbf{I}} \cdot 59 \mathbf{1} 68$ & 0.40832 & $\overline{1} .96$ & $\overline{\mathbf{I}} .57978$ & $\overline{\mathbf{r}} .61364$ & $0.3^{86} 636$ & $\overline{\mathbf{I}} .9$ & 40 \\
\hline 21 & 18 & 59205 & 95 & & & D & $\infty$ & & 39 \\
\hline 22 & 56150 & 59243 & 40757 & 96 & 58 & 61 & 38 & 96603 & $3^{8}$ \\
\hline 23 & 56 & & & & & 72 & $3^{8}$ & 98 & 37 \\
\hline 24 & 56215 & 59317 & 40683 & 96898 & $5^{8}$ & 08 & $3^{8} 492$ & 593 & 36 \\
\hline 25 & $\overline{1} .56247$ & $\overline{\mathrm{r}} \cdot 59354$ & 0.40646 & $\overline{\mathbf{I}} .96893$ & $\overline{\mathrm{I}} .58 \mathrm{I} 3 \mathrm{I}$ & $\overline{\mathbf{I}} .6 \mathrm{I} 544$ & $0.3^{8} 45^{6}$ & $\overline{\mathbf{I}} .9^{6} 588$ & 35 \\
\hline 26 & 56279 & $5939 I$ & 40609 & 96 & $5^{8}$ & 61579 & 38421 & $9^{6} 5^{82}$ & 34 \\
\hline 27 & 56311 & 59429 & 40 & & & 61615 & 38385 & 96577 & 33 \\
\hline 28 & 56343 & 59466 & 40 & 96878 & 23 & 51 & 38349 & 96572 & 32 \\
\hline 29 & 56 & 59503 & 40 & 3 & 3 & 7 & 3 & 7 & $3^{1}$ \\
\hline 30 & $\overline{\mathrm{I}} \cdot 56408$ & $\overline{\mathbf{I}} \cdot 59$ & 0.40460 & $\overline{\mathbf{I}} .96868$ & $\overline{\mathrm{I}} .58284$ & $\overline{\mathrm{I}} .6$ & 0.38278 & $\overline{\mathrm{I}} .96$ & 30 \\
\hline $3^{1}$ & 56 & 59 & 4 & 96863 & $5^{8}$ & 58 & $3 \varepsilon$ & $5^{6}$ & 29 \\
\hline 32 & 56 & $596 \mathbf{I} 4$ & 40 & $5^{8}$ & 58 & 4 & 38 & 9 & 28 \\
\hline 33 & 56 & $5965 \mathrm{I}$ & 40 & 53 & $5^{8}$ & 30 & 38 & 6 & 27 \\
\hline 34 & 56 & 5 & 2 & 8 & $5^{8}$ & 65 & 35 & I & 26 \\
\hline 35 & $\overline{\mathbf{I}} \cdot 56568$ & $\overline{\mathbf{I}} .59725$ & 0.40275 & $\bar{I} .96843$ & $\overline{\mathrm{I}} \cdot 5^{8} 436$ & $\overline{\mathrm{I}} .6 \mathrm{I} 90 \mathrm{I}$ & $0.3^{8}$ & $\overline{\mathbf{I}} .96$ & 25 \\
\hline $3^{6}$ & 56599 & 59762 & 4 & & $5^{8}$ & 61936 & & 0 & 24 \\
\hline 37 & 56631 & 59799 & & & $5^{8}$ & 72 & 28 & 96 & 3 \\
\hline 38 & 56663 & 59835 & 4 & 96 & & & 37 & & 2 \\
\hline 39 & 5 & 59 & & 3 & 57 & 43 & 57 & 14 & I \\
\hline 40 & I. 56727 & I.59909 & 0.40091 & $\overline{\mathbf{I}} .96$ & $\overline{\mathrm{I}} \cdot 5^{8} 5^{88}$ & $\overline{\mathbf{I}} .62$ & 0.37 & $\overline{\mathrm{I}} .9$ & 20 \\
\hline 41 & 59 & 59946 & & 968 & 58 & 62 & 36 & & 19 \\
\hline 42 & 5 & 33 & & & & 0 & & 8 & 18 \\
\hline 43 & 56 & 60019 & $3998 \mathbf{r}$ & 96 & $5^{8}$ & 62185 & 15 & & 17 \\
\hline 44 & 54 & 60056 & 39944 & 8 & 09 & 21 & 79 & 88 & 16 \\
\hline 45 & $\overline{\mathbf{I}} \cdot 56886$ & $\overline{\mathbf{1}} .60093$ & 0.39907 & $\overline{\mathbf{I}} .96$ & $\overline{\mathbf{x}} \cdot 5^{8} 739$ & $\overline{\mathbf{I}} .62256$ & 0.37 & $\overline{\mathbf{1}} .96483$ & 15 \\
\hline 4 & 56917 & 60130 & 39870 & 967 & $5^{8} 769$ & 62292 & 37 & 77 & 14 \\
\hline 47 & 56049 & 60166 & 39834 & & & 62 & & & 13 \\
\hline 48 & & 60203 & 39 & & & & & & 12 \\
\hline 49 & 57012 & 60240 & 39760 & $9677^{2}$ & 59 & 62398 & 37602 & 461 & II \\
\hline 50 & $\overline{\mathbf{I}} .57044$ & $\overline{\mathrm{I}} .60276$ & 0.39724 & $\overline{\mathbf{I}} .96$ & $\overline{\mathbf{I}} \cdot 5^{8889}$ & $\overline{\mathbf{I}} .6$ & 0.37 & $\overline{\mathbf{I}}$. & 10 \\
\hline 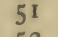 & 57075 & o 313 & 39687 & 62 & 58919 & 62468 & 37532 & 96451 & 9 \\
\hline $5^{2}$ & 57107 & o 349 & 39651 & 96 & $5^{8} 949$ & 62504 & & 45 & 8 \\
\hline 53 & 57138 & 60386 & 39 & & & & 61 & & 7 \\
\hline 54 & 57 & 60422 & 39578 & 96747 & 59009 & 62574 & 37426 & 96435 & 6 \\
\hline 55 & $\bar{x} \cdot 57201$ & $\overline{\mathbf{I}} .60459$ & $0.3954 \mathrm{I}$ & $\overline{\mathbf{I}} .96742$ & $\overline{\mathbf{I}} .59039$ & $\overline{\mathbf{I}} .62609$ & 0.37391 & $\overline{\mathbf{I}} .96429$ & 5 \\
\hline 5 & 57232 & & 39505 & 96737 & 59069 & 62645 & 37355 & 96424 & 4 \\
\hline & 57264 & 5532 & 39468 & 32 & 59098 & 62680 & 37 & 96 & 3 \\
\hline & 57295 & 60568 & 39432 & 96 & 59128 & 62715 & 37285 & 96413 & 2 \\
\hline \multirow{3}{*}{$60^{\prime}$} & 57326 & 60605 & 39395 & 96722 & 59 & $6275^{\circ}$ & 37250 & 96408 & I \\
\hline & $\overline{1} .5735^{8}$ & $\bar{I} .60641$ & 39359 & .96717 & $\overline{\mathrm{x}} .59$ I 88 & $\overline{\mathbf{I}} .62785$ & 0.37215 & $\overline{\mathbf{I}} .96403$ & $0^{\prime}$ \\
\hline & $\log \cos$ & $\log \cot$ & $\log \tan$ & $\log \sin$ & $\log \cos$ & $\log \cot$ & $\log \tan$ & $\log \sin$ & 1 \\
\hline
\end{tabular}




\begin{tabular}{|c|c|c|c|c|c|c|c|c|c|}
\hline 1 & $\log \sin$ & $\log \tan$ & $\log \cot$ & $\log \cos$ & $\log \sin$ & $\log \tan$ & $\log \cot$ & $\log 008$ & \\
\hline $0^{\prime}$ & $\overline{\mathbf{1}} .59188$ & $\overline{1} .627^{85}$ & 0.37215 & $\overline{\mathbf{1}} .96403$ & $\overline{1} .6093 \mathrm{I}$ & $\overline{\mathrm{I}} .6485^{8}$ & 0.35142 & $\overline{1} .96073$ & $60^{\prime}$ \\
\hline 1 & 59218 & 62820 & 37180 & 96397 & 60960 & 64892 & 35108 & 96067 & 59 \\
\hline 2 & 59247 & $628_{55}$ & $37 \quad 145$ & 96392 & 60988 & 64926 & 35074 & 96062 & 58 \\
\hline 3 & 59277 & 62890 & 37110 & 96387 & 61016 & 64960 & 35040 & 96056 & 57 \\
\hline 4 & 59307 & 62926 & 37074 & 96381 & 61045 & 64994 & 35006 & 96050 & 56 \\
\hline 5 & $\overline{\mathbf{I}} .59336$ & $\overline{\mathbf{I}} .62961$ & 0.37039 & I. 96376 & I. 61073 & $\overline{1} .65028$ & 0.34972 & ז. 96045 & 55 \\
\hline 6 & 59366 & 62996 & 37004 & 96370 & 61101 & 65062 & 34938 & 96039 & 54 \\
\hline 7 & 59396 & 63031 & 36969 & 96365 & 61129 & 65096 & 34904 & 96034 & 53 \\
\hline 8 & $59+25$ & 63066 & 36934 & 96360 & 61158 & 65130 & 34870 & 96028 & 52 \\
\hline 9 & 59455 & $63 \mathrm{ror}$ & 36899 & 96354 & 61186 & 65164 & 34836 & 96022 & $5^{1}$ \\
\hline 10 & $\overline{\mathbf{I}} .594_{4} 84$ & $\overline{1} .63135$ & 0.36865 & I. 96349 & $\bar{I} .61214$ & $\overline{\mathbf{I}} .65197$ & $0.3480_{3}$ & $\overline{1} .96017$ & 50 \\
\hline II & 59514 & 63170 & 36830 & 96343 & 61242 & 65231 & 34769 & 96011 & 49 \\
\hline 12 & 59543 & 63205 & 36795 & $9633^{8}$ & $6 \times 270$ & 65265 & 34735 & 96005 & 48 \\
\hline 13 & 59573 & 63240 & 36760 & 96333 & 61298 & 65299 & 34701 & 96000 & 47 \\
\hline 14 & 59602 & 63275 & 36725 & 96327 & 61326 & 65333 & 34667 & 95994 & 46 \\
\hline 15 & $\overline{\mathbf{1}} .59632$ & $\overline{1} .63310$ & 0.36690 & I. 96322 & $\bar{I} .61354$ & $\overline{1} .65366$ & 0.34634 & '. .95988 & 45 \\
\hline 16 & 59661 & 63345 & 36655 & 96316 & 61382 & 65400 & 34600 & 95982 & 44 \\
\hline 17 & 59690 & 63379 & 36621 & 96311 & 61411 & 65434 & 34566 & 95977 & 43 \\
\hline 18 & 59720 & 63414 & $365^{86}$ & 96305 & 61438 & 65467 & 34533 & 95971 & 42 \\
\hline 19 & 59749 & 63449 & $3655^{1}$ & 96300 & 61466 & 65501 & 34499 & 95965 & 41 \\
\hline 20 & $\overline{\text { I. }} 5977^{8}$ & I. 63484 & 0.36516 & I..96 294 & $\bar{I} .61494$ & T. 65535 & 0.34465 & I. 95960 & 40 \\
\hline 21 & 59808 & 63519 & 36481 & 96289 & 61522 & 65568 & 34432 & 95954 & 39 \\
\hline 22 & 59837 & 63553 & 36447 & 96284 & 61550 & 65602 & 34398 & 95948 & 38 \\
\hline 23 & $59 \$ 66$ & 63588 & 36412 & 96278 & $6157^{8}$ & 65636 & 34364 & 95942 & 37 \\
\hline 24 & 59895 & 63623 & 36377 & 96273 & 61606 & 65669 & 34331 & 95937 & 36 \\
\hline 25 & $\overline{\mathbf{I}} .59924$ & $\overline{1} .63657$ & 0.36343 & I. 96267 & $\overline{1} .61634$ & $\overline{1} .65703$ & 0.34297 & $\bar{I} .95931$ & 35 \\
\hline 26 & 59954 & 63692 & 36308 & 96262 & 61662 & 65736 & 34264 & 95925 & 34 \\
\hline 27 & 59983 & 63726 & 36274 & 96256 & 61689 & 65770 & 34230 & 95920 & 33 \\
\hline 28 & 60012 & 63761 & 36239 & 96251 & 61717 & 65803 & 34197 & 95914 & 32 \\
\hline 29 & 60041 & 63796 & 36204 & 96245 & 61745 & 65837 & 34163 & 95908 & 31 \\
\hline 30 & I. 60070 & i. 63830 & 0.36 I 70 & ธ̄. 96240 & $\overline{1} .61773$ & $\overline{1} .65870$ & 0.34130 & $\overline{1} .95902$ & 30 \\
\hline 31 & 60099 & 63865 & 36135 & 96234 & 61800 & 04 & 34 & 97 & 29 \\
\hline 32 & 60128 & 63899 & 36101 & 96229 & 61828 & 65937 & 34063 & $9589 \mathrm{i}$ & 28 \\
\hline 33 & 60157 & 63934 & 36066 & 96223 & 61856 & 65971 & 34029 & 95885 & 27 \\
\hline 34 & 60186 & 63968 & 36032 & 96218 & 61883 & 66004 & 33996 & 95879 & 26 \\
\hline 35 & $\overline{1} .60215$ & I. 64003 & 0.35997 & I. 96212 & I. 61911 & $\overline{1} .66038$ & 0.33962 & 1.95873 & 25 \\
\hline 36 & 602.44 & 64037 & 35963 & 96207 & 61939 & 66071 & 33929 & 95868 & 24 \\
\hline 37 & 60273 & 64072 & 35928 & 96201 & 61966 & 66104 & 33896 & 95862 & 23 \\
\hline 38 & 60302 & 64106 & 35894 & 96196 & 61994 & $6613^{8}$ & 33862 & 95856 & 22 \\
\hline 39 & 60331 & 64140 & 35860 & 96190 & 62021 & 66171 & 33829 & 95850 & 21 \\
\hline 40 & T.60 359 & $\overline{1} .64175$ & 0.35825 & ז. .96185 & T. 62049 & T. 66204 & 0.33796 & T. .95844 & 20 \\
\hline 41 & 60388 & 64209 & 35791 & 96179 & 62076 & $6623^{8}$ & 33762 & 95839 & 19 \\
\hline 42 & 60417 & 64243 & 35757 & 96174 & 62104 & 66271 & 33729 & 95833 & 18 \\
\hline 43 & $6044^{6}$ & 64278 & 35722 & 96168 & 62131 & 66304 & 33696 & 95827 & 17 \\
\hline 44 & 60474 & 64312 & 35688 & 96162 & 62159 & 66337 & 33663 & $9582 i$ & 16 \\
\hline 45 & I.60 503 & $i .64346$ & 0.35654 & I. 96157 & 1.62 186 & $\overline{1} .6637 \mathrm{I}$ & 0.33629 & 7.95815 & 15 \\
\hline 46 & 60532 & $643^{81}$ & 35619 & 96151 & 62214 & 66404 & 33596 & 95810 & 14 \\
\hline 47 & 60561 & 64415 & 35585 & 96146 & $6224 !$ & 66437 & $33^{6} 3$ & $95 \mathrm{SO}_{4}$ & 13 \\
\hline $4^{8}$ & 60589 & 64449. & $3555^{i}$ & 96140 & 62268 & 66470 & 33530 & 95798 & 12 \\
\hline 49 & 60618 & $644^{8} 3$ & 35517 & $96 \div 35$ & 62296 & $66 \mathrm{SO}_{3}$ & 33497 & 95792 & 11 \\
\hline 50 & T.60 6.46 & i. 64517 & $0.354^{8} 3$ & $\overline{1.96} 129$ & T. 62323 & 7.66537 & 0.3346 .3 & 7.95786 & 10 \\
\hline 51 & 60675 & 64552 & $3544^{8}$ & 96123 & 62350 & 66570 & 33430 & 957 so & 9 \\
\hline $5^{2}$ & 60704 & 64586 & 35414 & 96118 & 62377 & 66603 & 33397 & 95775 & 8 \\
\hline 53 & 60732 & 64620 & 35380 & 96112 & 62405 & $6663^{6}$ & 33364 & 95769 & 7 \\
\hline 54 & 60761 & 64654 & 35346 & 96107 & 62432 & 66069 & 33331 & $95>63$ & 6 \\
\hline 55 & T. 60789 & I. 64688 & 0.35312 & I. 96101 & T. $6 \geq 459$ & 7.66702 & 0.33208 & 7.95757 & 5 \\
\hline $5^{6}$ & $60 \$ 15$ & 64722 & $3527^{8}$ & 96095 & 62480 & 66735 & 33265 & $9575 i$ & 4 \\
\hline 57 & 608.46 & 64756 & 352.44 & $96 \mathrm{mo}$ & 62513 & 66768 & 33232 & 95745 & 3 \\
\hline $5^{8}$ & 60875 & 64790 & 35210 & 96084 & 62541 & $66 \mathrm{Sol}$ & $33(9)$ & 95739 & 2 \\
\hline 57 & 60903 & 64824 & 35176 & 96079 & 62568 & 66834 & 33166 & 95733 & 1. \\
\hline \multirow[t]{2}{*}{$60^{\prime}$} & 7.60031 & i. $\lim _{4} 858$ & 0.35142 & 1.96073 & 1.62505 & 1.66567 & 0.33133 & 1.95728 & $0^{\prime}$ \\
\hline & $\log \cos$ & $\log \cot$ & log tas & $\log$ ain & $\log c 08$ & log oot & $\log \tan$ & log $\sin$ & \\
\hline
\end{tabular}




\begin{tabular}{|c|c|c|c|c|c|c|c|c|c|}
\hline ! & $\log \sin$ & $\log \tan$ & $\log \cot$ & $\log \cos$ & $\log \sin$ & $\log \tan$ & $\log 00 t$ & $\log \cos$ & \\
\hline $0^{\prime}$ & $\overline{\mathbf{x}} .62595$ & $\overline{1} .60867$ & 0.33133 & $\overline{1} .95728$ & $\overline{1} .64184$ & $\overline{1} .68 \$ 15$ & 0.31182 & $\overline{1} .95366$ & $60^{\prime}$ \\
\hline I & 62622 & 66900 & 33100 & 95722 & 64210 & $68 S_{50}$ & 31150 & 95360 & 59 \\
\hline 2 & 62649 & 66933 & 33067 & 95716 & $6+236$ & $6 S S_{32}$ & 31118 & 95354 & $5^{8}$ \\
\hline 3 & 62676 & 66966 & 33034 & 95710 & 64262 & 68914 & 31086 & 95348 & 57 \\
\hline 4 & 62703 & 66999 & 33001 & 95704 & $642 S 8$ & 68946 & 31054 & 95341 & $5^{6}$ \\
\hline 5 & $\overline{1} .62730$ & I. 67032 & 0.32968 & $\overline{\mathrm{I}} .9569 \mathrm{~s}$ & $\overline{1} .64313$ & $\overline{\mathbf{I}} .68978$ & 0.31022 & $\overline{\mathbf{I}} .95335$ & 55 \\
\hline 6 & 62757 & 67065 & 32935 & 95692 & 64339 & 69010 & 30990 & 95329 & 54 \\
\hline 7 & 62784 & 67098 & 32902 & 95686 & 64365 & 69042 & $3095^{8}$ & 95323 & 3 \\
\hline 8 & 62811 & 67131 & 32869 & 95680 & $6+391$ & 69074 & 30926 & 95317 & $5^{2}$ \\
\hline 9 & 62838 & 67163 & 32 & 95 & 17 & & 30894 & 95310 & $5^{1}$ \\
\hline 10 & $\overline{1} .62865$ & $\overline{1} .67196$ & $0.32 \mathrm{SO}_{4}$ & I. 95668 & I. 64442 & $\overline{\mathrm{I}} .69$ I 38 & 0.30862 & $\overline{\mathrm{I}} .95304$ & 50 \\
\hline II & 62892 & 67229 & 32771 & 95663 & 64468 & 69170 & 30830 & & 49 \\
\hline 12 & 62918 & 67262 & $3273^{8}$ & 95657 & 64494 & 69202 & 30798 & & $4^{8}$ \\
\hline 13 & 62945 & 67295 & 32705 & 95651 & 64519 & 69234 & & & 47 \\
\hline 14 & 62972 & 67327 & 32673 & 95645 & 64545 & 69266 & 30734 & 95279 & 46 \\
\hline 15 & $\overline{\mathbf{I}} .62999$ & $\overline{\mathbf{I}} .67360$ & 0.32640 & $\overline{\mathrm{I}} .95639$ & $\overline{\mathbf{x}} .6457 \mathrm{I}$ & $\overline{\mathbf{1}} .69298$ & 0.30702 & $\overline{1} .95273$ & 45 \\
\hline 16 & 63026 & 67393 & 32607 & & 64596 & 69329 & 30671 & & 44 \\
\hline 17 & 63052 & 67426 & 32574 & 95627 & 64622 & $6936 i$ & 30639 & $9526 i$ & 43 \\
\hline 18 & 63079 & $6745^{8}$ & $3254^{2}$ & 95621 & 64647 & 69393 & 30607 & 95254 & 42 \\
\hline 19 & 63106 & 67491 & 32509 & 95615 & 64673 & 69425 & 75 & 8 & I \\
\hline 20 & $\overline{1} .63133$ & $\overline{1} .67524$ & 0.32476 & $\overline{\mathbf{I}} .95609$ & $\overline{\text { I. }} 64698$ & I. 69457 & 0.30543 & $\overline{\mathbf{I}} .95$ & 40 \\
\hline 21 & 63159 & 67556 & 32444 & 95603 & 64724 & 69488 & & 95236 & 39 \\
\hline 22 & 63186 & $675^{89}$ & 324 II & 95597 & 64749 & 69520 & 30480 & 95229 & $3^{8}$ \\
\hline 23 & 63213 & 67622 & 32378 & $9559 \mathrm{I}$ & 64775 & 69552 & 30448 & 23 & 37 \\
\hline 24 & 63239 & 67654 & $3^{2} 346$ & 85 & 64800 & $695^{84}$ & 30416 & 17 & 36 \\
\hline 25 & $\overline{1} .63266$ & $\bar{I} .67687$ & $0.3^{2} 3^{1} 3$ & $\overline{\mathbf{I}} .95579$ & $\overline{\mathrm{I}} .64826$ & $\overline{\mathbf{1}} .69615$ & $0.303^{8} 5$ & $\overline{\mathrm{I}} .95$ & 35 \\
\hline 26 & 63292 & 67719 & 32281 & 95573 & $6485^{1}$ & 69647 & 30353 & & 34 \\
\hline 27 & 63319 & $6775^{2}$ & 32248 & 95 & 64877 & 69679 & 30321 & 95 & 33 \\
\hline 28 & 63345 & 67785 & 32215 & I & 64902 & 69710 & 30290 & 2 & $3^{2}$ \\
\hline 29 & 63372 & 67817 & 32183 & 95555 & 64927 & 42 & 30258 & 85 & 31 \\
\hline 30 & ז. 63398 & $\overline{\mathbf{x}} .67850$ & 0.32150 & $\overline{\mathrm{I}} .95$ & $\overline{\mathrm{I}} .64953$ & $\overline{\mathbf{I}} .69774$ & 0.30226 & $\overline{\mathbf{I}} .9$ ? & 30 \\
\hline 31 & 63425 & 67882 & 32118 & & 64978 & 69805 & 30195 & 95 & 29 \\
\hline $3^{2}$ & $6345 \mathrm{I}$ & 67915 & 32085 & 95 & 65003 & 69837 & 30163 & 67 & 28 \\
\hline 33 & $6347^{8}$ & 67947 & 32053 & & 65029 & 69868 & 30132 & 60 & 27 \\
\hline 34 & 63504 & 67980 & 32020 & 95525 & 65054 & 69900 & 30100 & $95 \times 54$ & 26 \\
\hline 35 & $\overline{\mathrm{I}} .6353 \mathrm{I}$ & $\overline{1} .68$ o1 2 & 0.31988 & $\overline{\mathbf{I}} .95519$ & $\overline{\mathbf{1}} .65079$ & $\overline{\mathbf{I}} .69932$ & 0.30068 & $\overline{\text { I. }} .95148$ & 25 \\
\hline 36 & 557 & 68044 & 31956 & & 65 & 63 & 37 & 41 & 24 \\
\hline 37 & 63583 & 68077 & 31923 & 7 & 65130 & 69995 & 30005 & 5 & 23 \\
\hline $3^{8}$ & 63610 & 68109 & 31891 & 955 & 65155 & & 29974 & 95 & 22 \\
\hline 39 & 36 & 68142 & 31858 & 95494 & & $5^{8}$ & 29942 & 22 & $2 I$ \\
\hline 40 & $\overline{1} .63662$ & $\overline{\mathbf{I}} .68174$ & 0.31826 & $\overline{\mathbf{I}} .95488$ & $\overline{\mathbf{I}} .65205$ & $\overline{\mathbf{I}} \cdot 70089$ & 0.29911 & $\overline{\mathbf{I}} .95$ & 20 \\
\hline 41 & & 68206 & 31794 & & 65230 & 70121 & & & 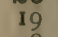 \\
\hline 42 & 5 & 68239 & 3 & & 5 & $3^{2}$ & 29848 & 3 & 18 \\
\hline 43 & $6374 \mathrm{I}$ & 8271 & 31729 & & 65281 & 70184 & 29816 & 95097 & 17 \\
\hline 44 & $\begin{array}{r}63767 \\
-\quad 5\end{array}$ & 303 & 31697 & & 65306 & 70215 & 29785 & 95090 & 16 \\
\hline 45 & $\overline{\mathrm{I}} .63794$ & $\overline{\mathbf{1}} .68336$ & 0.31664 & '. $.9545^{8}$ & $\overrightarrow{\mathrm{I}} .6533 \mathrm{I}$ & $\overline{\mathbf{I}} \cdot 70247$ & 0.29753 & $\bar{I} .95084$ & 15 \\
\hline 46 & 820 & 368 & 31632 & & 6 & & 22 & 78 & 14 \\
\hline 47 & 63846 & 68400 & 31600 & 95446 & $6538 I$ & 70309 & 29691 & 95071 & 13 \\
\hline 48 & 63872 & 68432 & 31568 & 40 & 66 & & 29 & 95 & 12 \\
\hline 49 & & $6_{5}$ & 31535 & 34 & 65431 & 70372 & & 95059 & II \\
\hline 50 & I. 63924 & I. 68497 & $0.3^{1} 503$ & $\bar{I} .95427$ & $\overline{1} .65456$ & $\overline{\mathbf{I}} \cdot 70404$ & 0.29596 & '̄. 95 & 10 \\
\hline $5^{1}$ & & & $3147 \mathrm{I}$ & & & & 65 & & 9 \\
\hline $5^{2}$ & 63976 & 68561 & $3^{1} 439$ & 95 & 65506 & 70466 & 29534 & $95 \circ 39$ & 8 \\
\hline 53 & & & $3^{1} 407$ & 95 & $6553 \mathrm{I}$ & 70498 & 29502 & 95033 & 7 \\
\hline 54 & & & $3^{1} 374$ & 95403 & 56 & 70529 & 29471 & 27 & 6 \\
\hline 55 & $\bar{I} .64054$ & $\overline{\mathbf{I}} .68658$ & 0.31342 & $\overline{\mathbf{I}} .95397$ & $\bar{I} .65580$ & $\overline{1} .70560$ & 0.29440 & $\overline{\mathbf{I}} .95020$ & 5 \\
\hline 56 & 64080 & 68690 & 31310 & 95391 & 65605 & 70592 & 29408 & 95 or 4 & 4 \\
\hline & 64106 & 68722 & 31278 & $953^{84}$ & 65630 & 70623 & 29377 & & 3 \\
\hline 58 & 64132 & 68754 & 31246 & & 65655 & 70654 & 29346 & $9500 \mathrm{i}$ & 2 \\
\hline & 64 I 58 & & 31214 & 95372 & 65680 & 70685 & 29315 & & $I$ \\
\hline \multirow[t]{2}{*}{$60^{\prime}$} & I. 64 I 84 & $\overline{1} .68818$ & 0.31182 & $\overline{\mathrm{I}} .95366$ & $\overline{\mathrm{I}} .65705$ & $\overline{1} .70717$ & 0.29283 & I. 94988 & $0^{\prime}$ \\
\hline & $\log \mathrm{cos}$ & $\log$ oot & $\log \tan$ & $\log \sin$ & $\log \cos$ & $\log \cot$ & $\log \tan$ & $\log \sin$ & \\
\hline
\end{tabular}




\begin{tabular}{|c|c|c|c|c|c|c|c|c|c|}
\hline 1 & $\log \sin$ & $\log \tan$ & $\log \cot$ & $\log 008$ & $\log \sin$ & $\log \tan$ & $\log \cot$ & $\operatorname{leg} \cos$ & \\
\hline $0^{\prime}$ & $\overline{1} .65705$ & 1.70717 & $0.292 \mathrm{~S}_{3}$ & $\overline{1} .94988$ & i. 67161 & $\overline{1} .72567$ & $0.27+33$ & $\overline{\mathbf{1}} .04593$ & $60^{\prime}$ \\
\hline I & 65729 & 70748 & 29252 & 94 & 67185 & & 27402 & & 59 \\
\hline 2 & 65754 & 70779 & 29221 & & 67208 & $72 i$ & 27372 & So & 58 \\
\hline 3 & 65779 & 70810 & 29190 & 94 & 67232 & 72659 & 27341 & 94573 & 57 \\
\hline 4 & $65 \mathrm{SO}_{4}$ & $70 S_{41}$ & 29159 & 94902 & 67256 & 72689 & $273^{11}$ & 94567 & $5^{6}$ \\
\hline 5 & Т. 65528 & $\overline{1} .70873$ & 0.29127 & $\overline{1} .94956$ & $\overline{1} .67280$ & $\overline{\mathbf{I}} .7^{2} 720$ & 0.27280 & $\overline{1} .94560$ & 55 \\
\hline 6 & 65853 & 70904 & 29) 096 & 94949 & 67303 & 72750 & 27250 & 94553 & 54 \\
\hline 7 & $658-8$ & 70935 & 29065 & 94943 & 67327 & $7^{2} 7^{50}$ & 27 & & 53 \\
\hline 8 & 65902 & 70966 & 29034 & $9493^{\circ}$ & $6735^{\circ}$ & 72811 & 27189 & 40 & $5^{2}$ \\
\hline 9 & 65927 & 70997 & $2 y 003$ & 94930 & 67374 & 72841 & 27159 & 94533 & $5^{1}$ \\
\hline 10 & T. 65952 & $\overline{1} .71028$ & 0.28972 & I. 94923 & ז. 67398 & $\overline{1} .72872$ & 0.27128 & T. 94526 & 50 \\
\hline II & $6597^{6}$ & 71059 & 28941 & & 67421 & & & & 49 \\
\hline 12 & 66001 & 71090 & 28910 & & 67445 & & & & 48 \\
\hline 13 & 66025 & 71121 & 28579 & & 67465 & & & & 47 \\
\hline 14 & 66050 & 71153 & 28847 & 94 & 67492 & 72993 & 27007 & 94499 & 46 \\
\hline 15 & $\overline{\mathbf{I}} .66075$ & $\overline{1} .71184$ & 0.28516 & $\overline{\mathbf{I}} .9489 \mathrm{I}$ & $\overline{1} .67515$ & $\overline{1.73023}$ & 0.26977 & โ. 94492 & 45 \\
\hline 16 & 6099 & 71215 & $2 S_{7} S_{5}$ & 85 & 67539 & & 26 & & 44 \\
\hline 17 & 66124 & 71246 & 25754 & $7^{8}$ & 67562 & $7308_{4}$ & 26 & & 43 \\
\hline 18 & 66148 & 71277 & 28723 & 94 & 67586 & & 26886 & 472 & 42 \\
\hline 19 & 66 & 71 & 92 & 5 & 9 & 44 & 56 & 465 & 41 \\
\hline 20 & I. 66197 & $\overline{1} .71$ & $6 I$ & $\overline{1} .94858$ & $\overline{1} .67633$ & $\overline{\mathbf{I}} .7$ & 0.2 & $45^{8}$ & 40 \\
\hline 21 & 62 & 71 & 30 & & 67 & & 95 & & 3) \\
\hline 22 & 6246 & 71 & 9 & & 67 & 35 & 26765 & & $3^{5}$ \\
\hline 23 & 60270 & 71431 & 28569 & & 67703 & & 26735 & & 37 \\
\hline 24 & 66395 & 714 & 28538 & & 67726 & & 05 & & 36 \\
\hline 25 & 1. 66319 & $\overline{\mathbf{I}} .71493$ & 0.28507 & I. 94 & $\overline{\mathrm{I}} .67750$ & $\overline{1} .73$ & 0.26674 & $\overline{1} .9$ & 35 \\
\hline 26 & 343 & $7^{1} 5^{24}$ & 28476 & 9 & 67 & & 26 & & 34 \\
\hline 27 & 368 & 71555 & 28445 & & 67796 & 73 & 26 & & 33 \\
\hline $\mathbf{2 8}$ & 66392 & 71586 & $28+14$ & 94 & 67820 & & 26 & & 32 \\
\hline 29 & 6416 & 71617 & 3 & & 3 & & 54 & & 31 \\
\hline 30 & I. $6644 \mathrm{I}$ & $\overline{\mathbf{I}} \cdot 71648$ & $0.2835^{2}$ & $\overline{\mathbf{1}} .9$ & ก. 67 & $\overline{\mathrm{I}} .73$ & 0.26524 & T. .9 & 30 \\
\hline $3 t$ & 65 & 71 & 28 & & 67 & & & & 20) \\
\hline $3^{2}$ & $5+89$ & 71709 & 28291 & & 67 & & 26 & & $2 S$ \\
\hline 33 & 66513 & 71740 & 28260 & & 67 & & 26433 & 69 & 27 \\
\hline 34 & 66537 & 71771 & 282 & & 9 & & 3 & & 26 \\
\hline 35 & $\overline{\mathrm{I}} .66562$ & $\overline{1} .71802$ & 0.28198 & $\overline{\mathbf{I}} .9$ & I. 67982 & $\overline{1} .73$ & 0.26373 & T. 9 & 25 \\
\hline 36 & 586 & 71833 & 28167 & & $68 c$ & & 26 & & 24 \\
\hline 37 & 610 & 71863 & 28137 & & & 87 & 26313 & 94342 & 23 \\
\hline $3^{5}$ & 634 & 71894 & 28106 & & $; 2$ & & 26283 & 35 & 22 \\
\hline 39) & 658 & $7^{1} 925$ & 75 & & 75 & & 26253 & & 21 \\
\hline 40 & I. 66682 & $\overline{1} .71$ & 0.28045 & I. .9 & $\overline{1} .68098$ & ก. 7. & 0.26 & $\overrightarrow{\mathrm{I}} .0$ & 20 \\
\hline 41 & 706 & $7^{1}$ & 28014 & & 21 & & & & 19 \\
\hline $4^{2}$ & 731 & $7^{2}$ & 983 & & 68144 & & 26163 & 94307 & 18 \\
\hline 43 & 755 & & $5^{2}$ & & & & & 94300 & 17 \\
\hline 44 & 6779 & $7^{2}$ & 922 & & 0 & & 3 & 93 & 16 \\
\hline 45 & T. 66803 & $\overline{1} \cdot 72109$ & 0.27891 & T. $94(x)_{4}$ & 1. 68213 & 1. 73 & 0.26073 & 1. .94250 & 15 \\
\hline $4 t$ & 827 & $7^{2} 140$ & 27860 & & 68237 & & 26043 & 94279 & 14 \\
\hline 47 & & 72170 & 27830 & & & & 26 & & 13 \\
\hline 48 & 5 & $7^{2}$ & 9 & & $S_{2} 2 S_{3}$ & & 3 & & 12 \\
\hline 49 & (9) & 72231 & 27769 & & 68305 & 47 & 25953 & 94259 & 11 \\
\hline 50 & 1. 66922 & ㄱ. 72262 & $0.2773^{8}$ & I. 9 & T. $6 \mathrm{~S} 32 \mathrm{~S}$ & I. 7 & 0.25 & T. 9 & 10 \\
\hline 51 & $6694^{6}$ & $7^{22} 293$ & & & 68351 & & $25 \mathrm{Sog}_{3}$ & & (1) \\
\hline $5^{2}$ & 66970 & 72323 & 27 & & 374 & & 25 & 94235 & 8 \\
\hline 53 & & 72354 & $27 c$ & & & & & 31 & 7 \\
\hline 54 & 7 ond & 72384 & 27616 & 94634 & 68420 & 74196 & $25 \mathrm{SO}_{4}$ & 24224 & 6 \\
\hline 55 & 1.67042 & 1.72415 & $0.275^{85}$ & i. 0.8627 & i. 68443 & 1. 74226 & 0.25774 & 1.04. 217 & 5 \\
\hline 5 & & 72445 & 27555 & 946320 & & & 25744 & 94210 & 4 \\
\hline 57 & $67(x) 0$ & $7^{2} 47$ & 27524 & & $\left.(6)_{4}+5\right)$ & & & & 3 \\
\hline $5^{8}$ & 78113 & $7^{2} 506$ & 27494 & 94607 & 68512 & 74316 & 25684 & 94106 & 2 \\
\hline & 67137 & 72537 & $274^{63}$ & 94600 & 65534 & 74345 & 25655 & $941 \mathrm{Sig}$ & 1 \\
\hline \multirow[t]{2}{*}{$60^{\prime}$} & $7.6716 \mathrm{i}$ & 1.72567 & 027433 & 1.04503 & 1.68557 & 1.74 375 & 0.25625 & 1.24182 & $0^{7}$ \\
\hline & $\log \mathrm{cos}$ & $\log$ cot & $\log \tan$ & logg sis & Iog cos & log ent & $\log$ tain & leg sin & , \\
\hline
\end{tabular}




\begin{tabular}{|c|c|c|c|c|c|c|c|c|c|}
\hline ' & $\log \sin$ & $\log \tan$ & $\log \cot$ & $\log \cos$ & $\log \sin$ & $\log \tan$ & $\log \cot$ & $\log \cos$ & \\
\hline $0^{\prime}$ & I. 65557 & 1.74375 & 0.25625 & $\overline{1} .94182$ & $\overline{1} .69897$ & $\overline{1} .76144$ & $0.23 \mathrm{~S}_{56}$ & $\overline{\mathbf{I}} .93753$ & $60^{\prime}$ \\
\hline 1 & 68550 & 74405 & 25595 & $94 \times 75$ & 69919 & 76173 & 23827 & & 59 \\
\hline 2 & 68603 & 74435 & 25565 & 94168 & 69941 & 76202 & 23798 & $9373^{8}$ & $5^{8}$ \\
\hline 3 & 68625 & 74465 & 25535 & 94161 & 69963 & 76231 & 23769 & & 57 \\
\hline 4 & 68648 & $7+494$ & 25506 & 94 I 54 & 69984 & 76261 & 23739 & & $5^{6}$ \\
\hline 5 & $\overline{\mathrm{I}} .6867 \mathrm{I}$ & $\overline{\mathrm{I}} .74524$ & 0.25476 & $\overline{\mathbf{1}} .94147$ & $\overline{\mathbf{I}} .70006$ & $\overline{\mathrm{I}} \cdot 76290$ & 0.23710 & $\overline{\mathbf{1}} \cdot 93$ & 55 \\
\hline 6 & 68694 & 74554 & $25+46$ & 94140 & 70028 & 76319 & 23681 & 93 & 54 \\
\hline 7 & 68716 & $745^{83}$ & 25417 & 94133 & 70050 & 76348 & 23652 & 93 & 53 \\
\hline 8 & 68739 & 74613 & 25387 & 94126 & 70072 & 76377 & 23623 & & $5^{2}$ \\
\hline 9 & 68762 & 74643 & 25357 & 94 II 9 & 70093 & & 23594 & 87 & $5^{1}$ \\
\hline 10 & ก. 68784 & $\overline{\mathrm{I}} .74673$ & 0.25327 & $\overline{1} .94112$ & $\overline{\mathrm{I}} .70115$ & $\overline{\mathrm{I}} .76435$ & 0.23565 & $\overline{\mathbf{I}} .93680$ & 50 \\
\hline II & $68 \mathrm{SO}_{7}$ & 74702 & 25298 & 94105 & 70137 & 76464 & 23536 & 93673 & 49 \\
\hline 12 & $68 \$ 29$ & 74732 & 25268 & 94098 & 70159 & 76493 & 23507 & & $4^{8}$ \\
\hline 13 & $68 S_{52}$ & 74762 & 25238 & 94090 & 70180 & 76522 & 23478 & & 47 \\
\hline 14 & $68 s_{75}$ & 74791 & 25209 & 94083 & 70202 & $7655^{1}$ & 23449 & 50 & 46 \\
\hline 15 & т.68897 & $\overrightarrow{\mathrm{I}} \cdot 7482 \mathrm{I}$ & 0.25179 & $\overline{\mathbf{I}} .94076$ & $\overline{\mathrm{I}} .70224$ & $\overline{\mathbf{I}} .76580$ & 0.23420 & $\overline{\mathbf{I}} .93643$ & 45 \\
\hline 16 & 68920 & 74851 & 25149 & 94069 & 70245 & 76609 & 23391 & 36 & 44 \\
\hline 17 & 68942 & 74880 & 25120 & 94062 & 70267 & 76639 & $233^{61}$ & & 43 \\
\hline 18 & 68965 & 74 & 25090 & 94055 & 70288 & 76668 & 23332 & 21 & 42 \\
\hline 19 & 68987 & 74 & 25 & 8 & 10 & 7 & $\mathrm{O} 3$ & 4 & 41 \\
\hline 20 & $\overline{\mathbf{I}} .69010$ & $\overline{\mathbf{I}} \cdot 74969$ & 0.25031 & $\bar{I} .94$ O4I & $\overline{\mathbf{x}} .70332$ & $\overline{\mathrm{x}} .76725$ & 0.23275 & $\overline{\mathbf{I}} .93606$ & 40 \\
\hline $2 I$ & 9032 & 74998 & 25002 & 34 & 70353 & 76 & 23246 & 99 & 39 \\
\hline 22 & 69055 & 75028 & 24972 & 94027 & 70375 & 76783 & 23217 & 91 & $3^{8}$ \\
\hline 23 & 69077 & $75 c$ & 24942 & 94020 & 70396 & 76812 & 23188 & 84 & 37 \\
\hline 24 & 69100 & 75 & 24913 & 94 OI 2 & 18 & 76841 & 23159 & 77 & 36 \\
\hline 25 & $\bar{I} .69122$ & $\overline{\mathbf{I}} .75117$ & 0.24883 & $\overline{\mathbf{I}} .94005$ & $\overline{\mathrm{I}} \cdot 70439$ & $\overline{\mathbf{I}} .76870$ & 0.23130 & $\overline{\mathbf{I}} .93$ & 35 \\
\hline 26 & 69144 & & 24854 & 93998 & 70461 & 76899 & 23 IOI & 62 & 34 \\
\hline 27 & 69167 & 75176 & 24824 & 93991 & 70482 & 76928 & 23072 & & 33 \\
\hline 28 & 69189 & 75205 & 24795 & 935 & 70 & 76957 & 23043 & & 32 \\
\hline 29 & 69212 & 75235 & 24765 & 93977 & 70 & 86 & 23014 & 39 & 3 I \\
\hline 30 & $\overline{1} .69234$ & $\overline{\mathbf{I}} .75264$ & 0.24736 & $\overline{\mathrm{I}} .93970$ & $\overline{\mathbf{I}} \cdot 70547$ & $\overline{\mathbf{I}} \cdot 77$ OI 5 & 0.22985 & $\overline{\mathrm{I}} .93$ & 30 \\
\hline $3^{1}$ & 256 & 75 & 24706 & 93963 & 70568 & 77 & 22956 & & 29 \\
\hline 32 & 69279 & 75323 & 24677 & 93955 & & 77 & & & 28 \\
\hline 33 & 69301 & 75353 & 24647 & 93948 & 70611 & 77 & 22899 & & 27 \\
\hline 34 & 69323 & 75382 & 24618 & 93 94I & 70633 & 77130 & 22870 & 2 & 26 \\
\hline 35 & $\overline{\mathbf{1}} .69345$ & $\overline{\mathrm{I}} \cdot 754 \mathrm{II}$ & $0.245^{89}$ & $\overline{\mathrm{I}} .93934$ & $\overline{\mathbf{I}} .70654$ & $\overline{\mathbf{x}} .77$ I 59 & $0.2284 \mathrm{I}$ & $\overline{\mathbf{T}} .93$ & 25 \\
\hline 36 & 8 & 7. & 24559 & & & & & & 24 \\
\hline 37 & 69390 & 75470 & 24530 & 93920 & 70697 & 77 & 22783 & 80 & 23 \\
\hline $3^{8}$ & 69412 & 75500 & 24500 & 93912 & 70718 & 77246 & 22754 & 72 & 22 \\
\hline 39 & 69434 & 75529 & 24471 & 93905 & 70739 & 77274 & 22726 & 65 & 21 \\
\hline 40 & $\overline{\text { I. }} 69456$ & $\overline{\mathbf{I}} .7555^{8}$ & 0.24442 & $\overline{\mathbf{1}} .93$ & $\overline{\mathbf{I}} \cdot 7076 \mathrm{I}$ & $\overline{\mathrm{I}} .77303$ & 0.22697 & $\overline{\mathrm{I}} . \mathrm{g}$ & 20 \\
\hline 41 & & 8 & 2 & 93 & 70782 & 77332 & 22668 & 93 & 19 \\
\hline 42 & OI & 75617 & 24383 & 93884 & 70803 & 773 & 22639 & 2 & 18 \\
\hline 43 & 523 & 75647 & 24353 & 93876 & 70824 & 77 & 22610 & 35 & I 7 \\
\hline 44 & 545 & 76 & 24324 & 69 & 70846 & 77418 & $225^{82}$ & 93427 & 16 \\
\hline 45 & I.69567 & I.75 705 & 0.24295 & I.93862 & $\overline{\mathbf{I}} .70867$ & $\overline{\mathbf{1}} \cdot 77447$ & 0.22553 & $\overline{\mathbf{I}} .93$ & I5 \\
\hline 46 & 589 & 75 & 24265 & 55 & 70888 & & 22524 & & 14 \\
\hline 47 & 611 & 75764 & 24236 & 93847 & 70 & 77 & 22495 & 93 & 13 \\
\hline $4^{8}$ & 633 & $75 \%$ & 24207 & & $70 \mathrm{c}$ & 77533 & 22467 & 97 & 12 \\
\hline 49 & 55 & & $2417^{8}$ & 33 & 70952 & $775^{62}$ & 22438 & 93390 & II \\
\hline 50 & $\overline{\mathbf{I}} .69677$ & $\overline{\mathrm{I}} .75852$ & 0.24148 & $\overline{\mathbf{I}} .93826$ & $\overline{\mathbf{1}} \cdot 70973$ & $\overline{\mathrm{r}} .775$ & 0.22409 & I. $933^{82}$ & 10 \\
\hline $5^{1}$ & & & 24119 & & 70 & 77 & $223^{81}$ & 75 & 9 \\
\hline $5^{2}$ & 69721 & 10 & 24090 & & 71 & 77648 & $2235^{2}$ & & 8 \\
\hline 53 & 69743 & 75939 & 24061 & 93804 & 710 & 77677 & 22323 & 360 & 7 \\
\hline 54 & & & 24031 & 93797 & 71058 & 77706 & 22294 & $9335^{2}$ & 6 \\
\hline 55 & $\overline{\mathbf{I}} .69787$ & $\overline{\mathbf{I}} \cdot 75998$ & 0.24002 & $\overline{\mathbf{I}} .93789$ & I.711079 & I.77 734 & 0.22266 & $\overline{\mathbf{I}} .93344$ & 5 \\
\hline 56 & 69809 & 76027 & 23973 & & 71100 & 77 & 22237 & & 4 \\
\hline 57 & 831 & 056 & 23944 & 93775 & 71121 & 77 & 22 & 93 & 3 \\
\hline 5 & & 086 & 23914 & & 71142 & 77820 & 22180 & & 2 \\
\hline 30 & 69875 & $76 \times 15$ & 23885 & 93760 & $\begin{array}{lll}71 & 163\end{array}$ & 77849 & 22 I $5^{I}$ & 93314 & I \\
\hline \multirow[t]{2}{*}{$60^{\prime}$} & $\overline{1} .69897$ & I. 76144 & 0.23856 & I.93753 & $\overline{\mathbf{1}} \cdot 71 \mathrm{1} 84$ & $\overline{\mathbf{1}} \cdot 77877$ & 0.22 I 23 & $\overline{\mathbf{x}} .93307$ & $0^{\prime}$ \\
\hline & $\log \cos$ & $\log \cot$ & $\log \tan$ & $\log \sin$ & $\log \cos$ & $\log \cot$ & $\log \tan$ & $\log \sin$ & 1 \\
\hline
\end{tabular}



LOG SIN, etc.

\begin{tabular}{|c|c|c|c|c|c|c|c|c|c|}
\hline ' & $\log \sin$ & $\log \tan$ & $\log \cot$ & $\log c 08$ & $\log \sin$ & $\log \tan$ & $\log 00 t$ & $\log \cos$ & \\
\hline $0^{\prime}$ & $\bar{I} .71184$ & $\overline{\mathrm{I}} .77877$ & 0.22123 & $\overline{1} .93307$ & $\overline{\mathrm{I}} \cdot 7^{2}+2 \mathrm{I}$ & $\overline{\mathbf{I}} .79579$ & $0.2042 \mathrm{I}$ & $\overline{1} .92 \mathrm{~S}_{42}$ & $60^{\prime}$ \\
\hline 1 & 71205 & 77906 & 22094 & 93299 & 72441 & 79607 & 20393 & $92 S_{34}$ & 59 \\
\hline 2 & 71226 & 77935 & 22065 & 93291 & $7^{2}+61$ & 79635 & 20365 & $92 \$ 26$ & $5^{8}$ \\
\hline 3 & 71247 & 77903 & 22037 & 93234 & $7^{2}+82$ & 79663 & 20337 & 92818 & 57 \\
\hline 4 & 71268 & 77992 & 22008 & 93276 & 72502 & 79691 & 20309 & 92 S10 & 56 \\
\hline 5 & $\bar{I} .71289$ & $\overline{1} .78020$ & 0.21980 & $\bar{I} .93269$ & $\bar{I} \cdot 7^{2} 522$ & $\overline{\mathbf{I}} .79719$ & 0.20281 & I. $92 \mathrm{SO}_{3}$ & 55 \\
\hline 6 & 71310 & 78049 & 21951 & $9326 i$ & $7^{2} 54^{2}$ & 79747 & 20253 & 92795 & 54 \\
\hline 7 & 71331 & 79077 & 21923 & 93253 & 72562 & 79776 & 20224 & 92787 & 53 \\
\hline 8 & 71352 & 78106 & 21894 & 93246 & $72 \mathrm{SS}_{2}$ & $79 \mathrm{SO}_{4}$ & 20196 & 92779 & $5^{2}$ \\
\hline 9 & 71373 & $7^{8} 135$ & 21865 & $9323^{8}$ & 72602 & 79832 & 20168 & 92771 & $5^{1}$ \\
\hline 10 & $\bar{I} .71393$ & I. 78163 & 0.21837 & $\overline{1} .93230$ & $\bar{I} .72622$ & $\overline{\mathbf{I}} .79860$ & 0.20140 & $\overline{1} .927^{6} 3$ & 50 \\
\hline 11 & 71414 & 78192 & 21508 & 93223 & 72643 & 79858 & 20112 & 92755 & 49 \\
\hline 12 & 71435 & 78220 & 21780 & 93215 & 72663 & 79916 & 20084 & 92747 & 48 \\
\hline 13 & $7145^{6}$ & $7^{8} 249$ & 21751 & 93207 & 72683 & 79944 & 20056 & 92739 & 47 \\
\hline 14 & 71477 & $7^{8} 277$ & 21723 & 93200 & 72703 & 79972 & 20028 & 92731 & 46 \\
\hline 15 & $\overline{1} .71498$ & $\overline{\mathbf{1}} .7^{9}, 306$ & 0.21694 & $\overline{1} .93192$ & 1.72723 & I. 80000 & 0.20000 & $\overline{1} .92723$ & 45 \\
\hline 16 & 71519 & 78334 & 21666 & $93 \times 84$ & 72743 & 80028 & 19972 & 92715 & 44 \\
\hline 17 & 71539 & $7^{8} 303$ & 21637 & 93177 & 72763 & 80056 & 19944 & 92707 & 43 \\
\hline 18 & 71500 & 78391 & 21609 & 93169 & $72-83$ & $8008_{4}$ & 19916 & 92699 & 42 \\
\hline 19 & 71581 & $78+19$ & $2 I 58 I$ & 93161 & 72803 & 80112 & I9 888 & 92691 & 41 \\
\hline 20 & I.71 602 & $\overline{1} \cdot 7^{8}+4^{8}$ & $0.2155^{2}$ & $\bar{I} .93154$ & $\bar{I} .72823$ & I. 80140 & 0.19860 & $\overline{1} .92683$ & 40 \\
\hline 21 & 71622 & $7^{8} 476$ & 21524 & 93146 & 72843 & 80168 & $198_{32}$ & 92675 & 39 \\
\hline 22 & 71643 & 78505 & 21495 & 93138 & 72863 & 80195 & $19 \mathrm{SO}_{5}$ & 92667 & 38 \\
\hline 23 & 71664 & 78533 & 21467 & 93 I 31 & $7288_{3}$ & 80223 & 19777 & 92659 & 37 \\
\hline 24 & 71685 & 78562 & $2143^{8}$ & 93123 & 72902 & SO $25^{1}$ & 19749 & 92651 & 36 \\
\hline 25 & $\overline{1} .71705$ & $\overline{\mathbf{I}} .79590$ & 0.21410 & $\overline{1} .93115$ & $\overrightarrow{1} .72922$ & $\overline{1} .80279$ & 0.19721 & $\overline{1} .92643$ & 35 \\
\hline 26 & 71726 & 78618 & 21382 & 93108 & 72942 & 80307 & 19693 & 92635 & 34 \\
\hline 27 & 71747 & 78647 & 21353 & 93100 & 72962 & 80335 & 19665 & 92627 & 33 \\
\hline 28 & 71767 & 78675 & 21325 & $93 \circ 92$ & 72982 & 80363 & 19637 & 92619 & 32 \\
\hline 29 & 71783 & 78704 & 21296 & 93084 & 73002 & 80391 & 19609 & 92611 & $3 I$ \\
\hline 30 & $\vec{I} .71809$ & i. 78732 & 0.21268 & $\bar{I} .9$ & $\overline{1} .73022$ & I. 80419 & $0.195^{81}$ & $\overline{1} .92603$ & 30 \\
\hline $3 I$ & $71 S 29$ & 78760 & 21240 & 93069 & 73041 & 80447 & 19553 & 92595 & 29 \\
\hline 32 & 71850 & $7^{5} 7^{59}$ & 21211 & 93061 & 73061 & 80474 & 19526 & $9^{2} 587$ & 28 \\
\hline 33 & 71870 & $78 \$ 17$ & 21183 & 93053 & 73081 & 80502 & 19498 & 92579 & 27 \\
\hline 34 & 71891 & $7^{88} 45$ & 21155 & 93046 & 73101 & 80530 & 19470 & $9^{2} 571$ & 26 \\
\hline 35 & $\overline{1} .71911$ & I. 78874 & 0.21126 & 1.93038 & I. 73121 & I. 80558 & 0.19442 & I. $.9^{2} 5^{6} 3$ & 25 \\
\hline $3^{6}$ & 71932 & 78902 & 21098 & 93030 & 73140 & 80586 & 19414 & 92555 & 24 \\
\hline 37 & 71952 & 78930 & 21070 & 93022 & 73160 & 80614 & 19386 & 92540 & 23 \\
\hline $3^{s}$ & 71973 & 78959 & 21041 & 93014 & 73180 & 80642 & $1935^{8}$ & $9^{2} 53^{8}$ & 22 \\
\hline 39 & 71994 & 78987 & 21013 & 93007 & 73200 & 80669 & 19331 & 92530 & 21 \\
\hline 40 & $\bar{I} .72014$ & $\overline{1} .79015$ & 0.20985 & I.92999 & $\overline{1} .73219$ & 97 & 0.19303 & $\overline{1} .92522$ & 20 \\
\hline 41 & 72034 & 79043 & 20957 & 92991 & 73239 & 80725 & 10275 & 92514 & 19 \\
\hline 42 & 72055 & 79072 & $20 y 28$ & 92 y3 3 & 73259 & 80753 & 19247 & 92506 & 18 \\
\hline 43 & 72075 & 79100 & 20,900 & 92976 & 73278 & 80781 & 19219 & 92498 & 17 \\
\hline 44 & -72006 & $\begin{array}{r}79128 \\
-\quad 1\end{array}$ & $20 S_{72}$ & 92968 & 73298 & So Sos & 19192 & 92490 & 16 \\
\hline 45 & ก. 72116 & i. 79156 & $0.208_{44}$ & I. 92960 & $\overline{1} .73318$ & T. 80836 & 0.19164 & $\bar{J} .924 S_{2}$ & 15 \\
\hline 46 & 72137 & $7918_{5}$ & $20 \mathrm{Sis}$ & $9295^{2}$ & 73337 & 80864 & 19136 & 92473 & 14 \\
\hline 47 & 72157 & 79213 & 20787 & 92944 & 73357 & 80892 & 19108 & 92465 & 13 \\
\hline $4^{8}$ & 72177 & 79) 241 & 20759 & 92936 & 73377 & 80919 & 19081 & 92457 & 12 \\
\hline 49 & 72198 & 79260 & 20731 & 92929 & 73396 & 80947 & 19053 & 92449 & 11 \\
\hline 50 & $\overline{1} .72218$ & i. 79297 & $0.2070_{3}$ & T. .92921 & T. 73416 & T. 80 y 75 & 0.10025 & T. 924.41 & 10 \\
\hline 51 & $7223^{8}$ & 79326 & 20674 & 42013 & 73435 & 81003 & 18997 & $92+3.3$ & 9 \\
\hline 52 & 72259 & 79354 & 20646 & 92005 & 73455 & 81030 & 18970 & 92425 & 8 \\
\hline 53 & 72270 & $793 S_{2}$ & 20618 & 92847 & 73474 & 81058 & 18042 & 92416 & 7 \\
\hline 54 & 72299 & 79410 & 20500 & 92854 & 73494 & 81086 & 18014 & 92408 & 6 \\
\hline 55 & I. 72320 & $7.794,38$ & 0.20562 & I. ${ }^{2} \mathrm{sSi}$ & I. 73513 & T. 81113 & $0.18 \mathrm{SS}_{7}$ & $\overline{1} .92400$ & 5 \\
\hline 56 & 72340 & $-94^{160}$ & 20534 & 92874 & 73533 & 81 $14 i$ & Is 850 & $y^{2} \cdot 302$ & 4 \\
\hline 57 & 72360 & 79495 & 20505 & 92866 & 73552 & $S_{1}(f(x)$ & $1 \mathrm{~S}_{3} 1$ & $923 \mathrm{~S}_{4}$ & 3 \\
\hline 58 & 72381 & i) 523 & 20477 & 9285.5 & 73572 & 81300 & $18 \mathrm{Sh}_{4}$ & $923 \pi^{-1}$ & 2 \\
\hline $5 \%$ & 72401 & 79551 & 20440 & 42850 & 73591 & 81224 & is 776 & 02367 & 1 \\
\hline \multirow[t]{2}{*}{$60^{\prime}$} & 1.724.21 & 1. $-7,5,7$ & $0.20+121$ & I. 928.42 & $1: 73611$ & I.S1 252 & 0.157 .45 & 1.02350 & $0^{\prime}$ \\
\hline & $\log \mathrm{cos}$ & $\log e 0 t$ & log tan & log bin & $\log$ cos & log eot & log tan & $\log \sin$ & \\
\hline
\end{tabular}




\begin{tabular}{|c|c|c|c|c|c|c|c|c|c|}
\hline ! & $\log \sin$ & $\log \tan$ & $\log \cot$ & $\log \cos$ & $\log \sin$ & $\log \tan$ & $\log \cot$ & $\log \cos$ & \\
\hline $0^{\prime}$ & $\overline{1} .73611$ & $\overline{1} .5125^{2}$ & 0.18748 & $\overline{1} .92359$ & $\overline{1} \cdot 7+75^{6}$ & $\overline{1} .82899$ & 0.17101 & $\overline{\mathbf{I}} .91857$ & $60^{\prime}$ \\
\hline I & 73630 & 81279 & 18721 & 92351 & 74775 & 82926 & 17074 & 91849 & 59 \\
\hline 2 & 73650 & 81307 & 18693 & $9^{2} 343$ & 74794 & 82953 & 170.47 & 91840 & $5^{8}$ \\
\hline 3 & 73669 & 81335 & 18605 & 92335 & 74812 & 82980 & 17020 & 91832 & 57 \\
\hline 4 & 73689 & 81362 & $1 \$ 6,3$ & 92326 & 74831 & 83008 & 16992 & 91823 & 56 \\
\hline 5 & $\overline{1} .73$ 708 & $\overline{\mathbf{I}} .81390$ & 0.18610 & $\bar{I} .92318$ & $\overline{\mathrm{I}} \cdot 748_{50}$ & $\overline{1} .83035$ & 0.16965 & $\bar{I} .91815$ & 55 \\
\hline 6 & 73727 & 81418 & 18582 & 92310 & 74568 & 83062 & 16938 & 91806 & 54 \\
\hline 7 & 73747 & 81445 & 18555 & 92302 & 74887 & 83089 & 16911 & 91798 & 53 \\
\hline 8 & 73766 & 81473 & 18527 & 92293 & 74906 & 83117 & 16883 & 91789 & 52 \\
\hline 9 & 73785 & 81500 & 18500 & 92285 & 74924 & 83144 & I 6856 & 91781 & $5 I$ \\
\hline 10 & $\overline{1} .73 \mathrm{SO}_{5}$ & I. 81528 & $0.1847^{2}$ & $\bar{I} .92277$ & $\overline{\mathbf{I}} .74943$ & $\overline{1} .83 \mathrm{I} 7 \mathrm{I}$ & 0.16829 & $\overline{\mathbf{I}} .9177^{2}$ & 50 \\
\hline II & 73824 & 81556 & IS 444 & 92269 & 74961 & 83198 & 16802 & 91763 & 49 \\
\hline 12 & $738+3$ & 81583 & 18417 & 92260 & 74980 & 83225 & 16775 & 91755 & 48 \\
\hline 13 & $73 \mathrm{S6}_{3}$ & 816 I I & 18389 & 92252 & 74999 & 83252 & 16748 & 91746 & 47 \\
\hline 14 & $73 \mathrm{SS2}$ & 81638 & 18362 & 92244 & 75017 & 83280 & 16720 & 91738 & 46 \\
\hline 15 & $\overline{\mathbf{I}} \cdot 73901$ & $\overline{\mathrm{I}} .8 \mathrm{r} 666$ & 0.18334 & I. .92235 & $\overline{\mathbf{I}} \cdot 75 \circ 36$ & $\overline{1} .83307$ & 0.16693 & $\bar{I} .91729$ & 45 \\
\hline 16 & 73921 & 81693 & 18307 & 92227 & 75054 & 83334 & 16666 & 91720 & 44 \\
\hline 17 & 73940 & 81721 & 18279 & 92219 & 75073 & 83361 & 16639 & 91712 & 43 \\
\hline 18 & 73959 & $8174^{8}$ & 18252 & 92211 & 75 og I & 83388 & 16612 & 91703 & $4^{2}$ \\
\hline 19 & 73978 & $8177^{6}$ & I 8224 & 92202 & 75110 & 83415 & $165^{8} 5$ & 91695 & $4 I$ \\
\hline 20 & $\overline{\text { I.73 }} 997$ & $\overline{1} .81803$ & 0.18197 & $\bar{I} .92194$ & $\overline{\mathbf{I}} .75128$ & $\overline{\mathbf{I}} .83442$ & $0.1655^{8}$ & $\overline{\text { I. }} .91686$ & 40 \\
\hline 21 & 74017 & 81831 & 18169 & 92186 & 75147 & 83470 & 16530 & 91677 & 39 \\
\hline 22 & 74036 & 81858 & I 8142 & 92177 & 75165 & 83497 & $1650_{3}$ & 91669 & $3^{8}$ \\
\hline 23 & 74055 & 81886 & 18 II 4 & 92169 & $75 \times 84$ & 83524 & 16476 & 91660 & 37 \\
\hline 24 & 74074 & 81913 & 18087 & 92 I 61 & 75202 & 8355 I & 16449 & 91651 & 36 \\
\hline 25 & $\overline{\mathbf{I}} .74093$ & $\overline{\mathrm{I}} .81941$ & 0.18059 & $\overline{\mathrm{I}} .9215^{2}$ & $\overline{\mathrm{I}} \cdot 7522 \mathrm{I}$ & $\overline{\mathrm{I}} .83578$ & o. 16422 & $\overline{1} .91643$ & 35 \\
\hline 26 & 74 II 3 & 81968 & 18032 & 92144 & 75239 & 83605 & I6 395 & 91634 & 34 \\
\hline 27 & 74132 & 81996 & I 8004 & 92 I 36 & 75258 & 83632 & 16368 & 91625 & 33 \\
\hline 28 & 74 I 51 & 82023 & I7 977 & 92127 & 75276 & 83659 & I 634 I & 91617 & 32 \\
\hline 29 & 74170 & 82051 & I 7949 & 92119 & 75294 & 83686 & 16314 & 91608 & $3 I$ \\
\hline 30 & $\overline{\mathbf{I}} .74189$ & $\overline{\mathbf{I}} .82078$ & 0.17922 & $\bar{I} .92$ I I I & $\overline{1} .75313$ & $\overline{1} .83713$ & 0.16287 & $\overline{\mathbf{I}} .91599$ & 30 \\
\hline $3 I$ & 74208 & 82106 & 17894 & 92102 & 7533 I & 83740 & 16260 & 91591 & 29 \\
\hline 32 & 74227 & 82 I33 & 17867 & 92094 & 75350 & 83768 & 16232 & 91582 & 28 \\
\hline 33 & 74246 & $82+61$ & 17839 & 92086 & 75368 & 83795 & 16205 & 9I 573 & 27 \\
\hline 34 & 74265 & $\begin{array}{r}82 \text { I } 88 \\
-\end{array}$ & 17812 & 92077 & 75386 & 83822 & 16178 & 91565 & 26 \\
\hline 35 & $\overline{\mathbf{I}} .74284$ & $\overline{1} .82215$ & 0.17785 & $\overline{\mathbf{I}} .92069$ & $\overline{\mathrm{I}} .75405$ & $\overline{\mathbf{I}} .83849$ & 0.16151 & $\overline{\mathbf{I}} .9155^{6}$ & 25 \\
\hline $3^{6}$ & 74303 & 82243 & 17757 & 92060 & 75423 & 83876 & 16 I 24 & 91547 & 24 \\
\hline 37 & 74322 & 82270 & 17730 & $9205^{2}$ & $7544 I$ & 83903 & 16097 & $9^{1} 53^{8}$ & 23 \\
\hline 38 & $7434 \mathrm{I}$ & 82298 & 17702 & 92044 & 75459 & 83930 & 16070 & 91530 & 22 \\
\hline 39 & 74360 & 82325 & 17675 & 92035 & $7547^{8}$ & 83957 & & 9 I 52 I & 21 \\
\hline 40 & $\overline{\mathbf{I}} .74379$ & $\overline{1} .82352$ & 0.17648 & $\overline{\mathbf{I}} .92027$ & $\overline{\mathbf{I}} .75496$ & $\overline{\mathbf{I}} .83984$ & 0.16 & $\bar{I} .91512$ & 20 \\
\hline $4 I$ & 74398 & 82380 & 17620 & 92018 & 755 I4 & II & I 5989 & 91504 & 19 \\
\hline 42 & 74417 & 82407 & 17593 & 92 oro & 75533 & 84038 & I 5962 & 91495 & 18 \\
\hline 43 & 74436 & 82435 & 17565 & 92002 & $7555 \mathrm{I}$ & 84065 & I 5935 & 91486 & 17 \\
\hline 44 & 74455 & 82462 & $1753^{8}$ & 91993 & 75569 & $84 \circ 092$ & 15908 & 91477 & 16 \\
\hline 45 & $\overline{\mathrm{I}} .74474$ & $\overline{\mathrm{I}} .82489$ & 0.17511 & $\bar{I} .91985$ & $\overline{\mathbf{I}} .755^{87}$ & $\overline{\mathbf{I}} .84$ I1 9 & 0.15881 & $\overline{\mathbf{I}} .91469$ & 15 \\
\hline 46 & 74493 & 82517 & 17483 & 91976 & 75605 & 84146 & I5 854 & 91460 & 14 \\
\hline 47 & 74512 & 82544 & 17456 & 91968 & 75624 & 84173 & I 5827 & 9 I 45 I & 13 \\
\hline $4^{8}$ & 74531 & 82571 & 17429 & 91959 & 75642 & 84200 & 15800 & 91442 & 12 \\
\hline 49 & 74549 & 82599 & 17401 & 9 I 95 I & 75660 & 84227 & 15 773 & 91433 & I I \\
\hline 50 & $\overline{\mathbf{I}} \cdot 74568$ & $\overline{\mathrm{I}} .82626$ & 0.17374 & $\bar{I} .91942$ & $\overline{\mathbf{I}} .75678$ & $\overline{1} .84254$ & 0.15746 & $\overline{\text { I. }} .91425$ & 10 \\
\hline 51 & $745^{87}$ & 82653 & 17347 & . 91934 & 75696 & 84280. & 15720 & 91416 & 9 \\
\hline $5^{2}$ & 74606 & 82681 & 17319 & 91925 & 75714 & 84307 & . I5 693 & 91407 & 8 \\
\hline 53 & 74625 & 82708 & 17292 & 91917 & 75733 & 84334 & I5 666 & 91398 & 7 \\
\hline 54 & 74644 & 82735 & 17265 & 91908 & $7575^{I}$ & $843^{6 I}$ & 15639 & 91389 & 6 \\
\hline 55 & $\overline{\mathrm{I}} \cdot 74662$ & $\overline{\mathrm{I}} .82762$ & o.17238 & $\overline{\mathbf{I}} .91900$ & $\overline{\mathbf{I}} \cdot 75769$ & $\overline{\mathbf{I}} .84388$ & 0.15612 & $\overline{\mathbf{I}} .9138 \mathrm{I}$ & 5 \\
\hline $5^{6}$ & $7468 I$ & 82790 & 17210 & 91891 & 75787 & 84415 & 15585 & 91372 & 4 \\
\hline 57 & 74700 & 82817 & 17183 & $9188_{3}$ & 75805 & 84442 & I 5558 & 91363 & 3 \\
\hline $5^{8}$ & 74719 & 82844 & 17156 & 91874 & 75823 & 84469 & 15531 & 91354 & 2 \\
\hline 59 & 74737 & -82871 & 17129 & 91866 & 75841 & 84496 & 15504 & 91345 & I \\
\hline \multirow[t]{2}{*}{$60^{\prime}$} & 1.74756 & $\overline{1} .82899$ & 0.17101 & $\overline{\mathbf{I}} .91857$ & $\overline{1} .75859$ & $\overline{1} .845^{23}$ & 0.15477 & 1.91336 & $0^{\prime}$ \\
\hline & $\log \cos$ & $\log \cot$ & $\log \tan$ & $\log \sin$ & $\log \cos$ & $\log \cot$ & $\log \tan$ & $\log \sin$ & $?$ \\
\hline
\end{tabular}




\begin{tabular}{|c|c|c|c|c|c|c|c|c|c|}
\hline ' & $\log \sin$ & $\log \tan$ & $\log \cot$ & $\log \cos$ & $\log \sin$ & $\log \tan$ & $\log \cot$ & $\log \cos$ & \\
\hline $0^{\prime}$ & $\overline{\mathbf{I}} .75859$ & 1.84523 & 0.15477 & $\overline{1} .91336$ & $\overline{\mathrm{I}} \cdot 76922$ & 1. 86126 & $0.13 \times 74$ & $1.907,96$ & $60^{\prime}$ \\
\hline 1 & 75877 & $8+550$ & 15450 & 91328 & 76939 & 86153 & $13 \times 47$ & yo 757 & 59 \\
\hline 2 & 75895 & $8+576$ & 15424 & 91319 & 76957 & 86179 & $13 S_{21}$ & 90777 & $5^{s}$ \\
\hline 3 & 75913 & $8+603$ & 15397 & 91310 & 76974 & 86206 & 13794 & 90765 & 57 \\
\hline 4 & 75931 & 84630 & 15370 & 91301 & 76991 & 86232 & 13768 & 90759 & 56 \\
\hline 5 & -.75949 & i. 84657 & 0.15343 & $\overline{1} .91202$ & $\overline{1} .77009$ & $\overrightarrow{\text { I. }} .86=59$ & 0.13741 & I. 90750 & 53 \\
\hline 6 & 75967 & 84684 & 15316 & 91283 & 77026 & 86285 & 13715 & 90741 & 54 \\
\hline 7 & 75983 & $8+7^{11}$ & 15280 & 91274 & 77043 & $863^{12}$ & 13658 & $9073 \mathrm{I}$ & 53 \\
\hline 8 & 76003 & 84735 & 15262 & 91266 & 77061 & $86,33^{8}$ & 13662 & 90722 & $5^{2}$ \\
\hline 9 & 76021 & 84764 & 15236 & 91257 & $7707^{8}$ & 86365 & 13635 & 90713 & 51 \\
\hline 10 & $\overline{1} .76039$ & $\overline{1} .84791$ & 0.15209 & $\overline{1} .91248$ & ז. 77095 & $\overline{7} .86392$ & 0.13608 & $\overline{1} .90704$ & 50 \\
\hline 11 & 76057 & 84818 & 15182 & 91239 & 77112 & $86+18$ & 13552 & 90604 & 49 \\
\hline 12 & 76075 & $848_{45}$ & 15155 & 91230 & 77130 & 86445 & 13555 & 90655 & 48 \\
\hline 13 & 76093 & 84872 & 15128 & 91221 & 77147 & 86471 & 13529 & 90676 & 47 \\
\hline 14 & 76111 & 84899 & 15101 & 91212 & 77164 & 86498 & 13502 & 90667 & 46 \\
\hline 15 & $\overline{1} .76129$ & $\overline{\mathbf{I}} .8+9^{25}$ & 0.15075 & $\overline{1} .91203$ & $\overline{1} \cdot 7718 \mathrm{I}$ & I. 86524 & 0.13476 & I. 90657 & 45 \\
\hline 16 & 76146 & 84952 & 15048 & 91194 & 77199 & 86551 & 13449 & 90648 & 44 \\
\hline 17 & 76164 & 84979 & 15021 & 91185 & 77216 & 86577 & 13423 & 90639 & 43 \\
\hline 18 & 76182 & 85006 & 14994 & 91176 & 77233 & $8660_{3}$ & 13397 & 90630 & 42 \\
\hline 19 & 76,200 & 85033 & 14967 & 91167 & 77250 & 86630 & 13370 & 90620 & 41 \\
\hline 20 & $\overline{1} .76218$ & $\overline{1} .85059$ & 0.14941 & $\overline{\mathbf{1}} .9115^{8}$ & $\overline{1} \cdot 77268$ & г. 86656 & 0.13344 & $\overline{1} .90611$ & 40 \\
\hline 21 & 76236 & 85086 & 14914 & 91149 & $7728_{5}$ & $8668_{3}$ & 13317 & 9062 & 39 \\
\hline 22 & 76253 & $8_{5} 1_{1}$ & 14857 & 91141 & 77,302 & 86709 & $1329 \mathrm{i}$ & 90592 & 38 \\
\hline 23 & 76271 & 85140 & 14860 & 91132 & 77319 & 86736 & 13264 & 90583 & 37 \\
\hline 24 & 76289 & 85166 & 14834 & 91123 & $7733^{6}$ & 86762 & 13238 & 90574 & 36 \\
\hline 25 & $\overline{\mathbf{I}} \cdot 7^{6} 307$ & $\overline{1} .85193$ & 0.14807 & $\overline{1} .91114$ & $\overline{1} .77353$ & I. $86 ; 89$ & 0.13211 & $\overline{1} .905_{5} 65$ & 35 \\
\hline 26 & $7^{6} 324$ & 85220 & 14780 & 91105 & $7737^{\circ}$ & 86815 & 13185 & 90555 & 34 \\
\hline 27 & 76342 & 85247 & 14753 & 91096 & 77387 & 86842 & 13158 & 90546 & 33 \\
\hline 28 & $7^{6} 360$ & 85273 & 14727 & 91087 & 77405 & 86568 & 13132 & 90537 & 32 \\
\hline 29 & $7637^{8}$ & 85300 & 14700 & 91078 & 77422 & $86 \mathrm{Sg}_{4}$ & 13106 & 90527 & 31 \\
\hline 30 & i. 76395 & I. 85327 & 0.14673 & $\overline{\mathbf{1}} .91069$ & $\overline{\mathbf{I}} .77439$ & $\overline{\mathrm{I}} .8692 \mathrm{I}$ & 0.13079 & $\overline{\mathrm{I}} .90518$ & 30 \\
\hline 31 & $7^{6}+13$ & 85354 & 14646 & 91060 & $7745^{6}$ & 86947 & 13053 & yo 509 & 20 \\
\hline 32 & $7^{6} 43^{1}$ & 85380 & 14620 & 91051 & 77473 & 86974 & 1,3026 & 90499 & 28 \\
\hline 33 & $7644^{8}$ & 85407 & 14593 & 91042 & 77490 & 87000 & 13000 & 90490 & 27 \\
\hline 34 & $7^{6} 4^{66}$ & 85434 & 14566 & 91033 & 97507 & 87027 & 12973 & 90450 & 26 \\
\hline 35 & T. $76{ }_{4} s_{4}$ & I. 85460 & 0.14540 & $\overline{1} .91023$ & I. 77524 & T.S7 053 & 0.12947 & $\overline{1} .90471$ & 25 \\
\hline 36 & 76501 & 85487 & 14513 & 91014 & 77541 & 87079 & 12921 & 90462 & 24 \\
\hline 37 & 76519 & $855^{14}$ & 14486 & 91005 & $7755^{8}$ & 87106 & $12 \mathrm{~S}_{1} 4$ & 90452 & 23 \\
\hline $3^{8}$ & 76537 & 85540 & 14450 & 90996 & 77575 & 87132 & $12 \operatorname{ses}$ & 90443 & 22 \\
\hline 39 & 76554 & 85567 & 14433 & 90987 & 77592 & $8715^{8}$ & $12 S_{42}$ & 904.34 & 21 \\
\hline 40 & $\overline{\text { I. }} \cdot 7^{6} 57^{2}$ & $\overline{\mathbf{1}} .85594$ & 0.14406 & $\overline{\mathbf{I}} .90978$ & $\overline{1} \cdot 7 \overline{7}(609)$ & $\overline{\mathrm{I}} \mathrm{S}_{7} \mathrm{IS}_{5}$ & $0.12 \mathrm{SI}_{15}$ & I. 90424 & 20 \\
\hline 41 & $7^{6} 590$ & 85620 & 14380 & $900(x)$ & 77626 & 87211 & $127 \mathrm{Siq}$ & 90415 & 19 \\
\hline 42 & $7^{6}, 1007$ & 85647 & 14353 & 90960 & 77643 & 872,35 & 12762 & 90405 & 18 \\
\hline 43 & 76625 & 85674 & 14326 & 90951 & 77660 & 87264 & $1273^{6}$ & 90306 & 17 \\
\hline 44 & 76642 & 85700 & 14300 & 90942 & 77677 & 87200 & 12710 & 90356 & 16 \\
\hline 45 & $7,7^{6}, 660$ & i.S5 727 & 0.14273 & T. .9093 .3 & 1.77(604 & 1.87317 & $0.1268_{3}$ & i. 90377 & 15 \\
\hline $4^{6}$ & 76677 & 85754 & 14246 & 90024 & 77711 & 87343 & 12657 & 90365 & 14 \\
\hline 47 & 76695 & 85750 & 14220 & 90915 & 77728 & $873^{(x)}$ & 12631 & $4035^{8}$ & 13 \\
\hline $4 s$ & 76,712 & $85 \mathrm{SO}_{7}$ & 14193 & 90006 & 77744 & 87390 & 12604 & $90.34 ?$ & 12 \\
\hline 49 & $7^{6} 730$ & 85834 & 14166 & 90896 & 77761 & 87422 & $1257 \mathrm{~S}$ & 90339 & 11 \\
\hline 50 & 1. 76747 & $\overline{1} .85860$ & 0.14140 & $\mathbf{1} .90 \mathrm{SS}_{7}$ & 7.77778 & I. 87.44 & 0.12552 & T. .003 .30 & 10 \\
\hline $5 t$ & 76765 & $S_{5} \mathrm{S8}_{7}$ & 14113 & yo $87 x^{\circ}$ & 77795 & 87475 & 12525 & 90,320 & ) \\
\hline $5^{2}$ & $7^{6} 7^{82}$ & $8_{5} 913$ & 14087 & $90 \sin$ & 77512 & $8>501$ & 12400 & 90311 & 8 \\
\hline 53 & 76800 & 85940 & 14060 & $90 \mathrm{~S}(x)$ & 77820 & $s 7527$ & 12473 & $90 \mathrm{zor}$ & 7 \\
\hline 54 & 76817 & 85967 & 14033 & 90851 & 778.40 & 87554 & 12446 & $90 \mathrm{202}$ & 6 \\
\hline 55 & Т. 76835 & T.85993 & 0.14007 & $i .008_{42}$ & i. 77862 & 1.87550 & 0.12420 & 1.002 .52 & 5 \\
\hline $5^{6}$ & 76852 & 86020 & 13950 & 20832 & 77599 & 87 io6 & 12324 & 90273 & 4 \\
\hline 57. & $7^{65} 70$ & 86046 & 13954 & $\cos _{23}$ & $77 \sin 6$ & $8 ; 633$ & 12,367 & yo 213 & 3 \\
\hline-8 & 76857 & 86073 & 13927 & 20814 & 77213 & $8765 ?$ & 12341 & 90254 & 2 \\
\hline & 76904 & 56100 & $13(x)$ & (x) sos & 77930 & $8-65$ & 12315 & 90244 & 1 \\
\hline \multirow[t]{2}{*}{$60^{\prime}$} & $7.76 \% 22$ & I.S6, 126, & $0.138-4$ & 190706 & 1.770 .46 & $\overline{\mathrm{I}} \mathrm{S} ; 7 \mathrm{i}$ & 0.12250 & 7.90235 & $0^{\prime}$ \\
\hline & $\operatorname{lng} 608$ & $\log \cot$ & log tan & logg nin & $\log \mathrm{cen}$ & log ent & lar tan & Tengsing & . \\
\hline
\end{tabular}




\begin{tabular}{|c|c|c|c|c|c|c|c|c|c|}
\hline , & $\log \sin$ & $\log \tan$ & $\log \cot$ & $\log \cos$ & $\log \sin$ & $\log \tan$ & $\log \cot$ & $\log \cos$ & \\
\hline $0^{\prime}$ & $\overline{1} .77946$ & $\overline{1} .87711$ & 0.12259 & $\overline{1} .90235$ & $\overline{\mathbf{I}} \cdot 7^{S 934}$ & $\overline{1} .89281$ & 0.10719 & I.89653 & 60 \\
\hline I & 77963 & 87738 & 12262 & 90225 & 78950 & 89307 & 10693 & 89643 & 59 \\
\hline 2 & 77950 & 87764 & 12236 & 90216 & 78967 & 89333 & 10667 & 89633 & $5^{8}$ \\
\hline 3 & 77997 & 87790 & 12210 & 90206 & 78983 & 89359 & $1064 \mathrm{I}$ & 89624 & 57 \\
\hline 4 & 78013 & 87817 & 12183 & 90197 & 78999 & 89385 & 10615 & 89614 & 56 \\
\hline 5 & $\overline{\mathbf{I}} .78030$ & $\overline{1} .87843$ & 0.12157 & $\overline{\mathbf{I}} .90187$ & $\overline{1} .79015$ & $\bar{I} .89+11$ & 0.10589 & $\overline{1} .89604$ & 55 \\
\hline 6 & 78047 & 87869 & 12131 & 90178 & $7903 \mathrm{I}$ & 89437 & $105^{6} 3$ & 89594 & 54 \\
\hline 7 & 78063 & 87895 & 12105 & 90168 & 79047 & 89463 & 10537 & 89584 & 53 \\
\hline 8 & 78 o80 & 87922 & 12078 & 90159 & 79063 & 89489 & 10511 & 89574 & 52 \\
\hline 9 & 78097 & $8794^{8}$ & 12052 & 90149 & 79079 & 89515 & 10485 & 89564 & 51 \\
\hline 10 & $\overline{1} .78113$ & $\overline{\mathbf{I}} .87974$ & 0.12026 & $\bar{x} .90139$ & $\overrightarrow{\mathbf{I}} \cdot 79095$ & $\overline{\mathbf{I}} .89541$ & 0.10459 & $\overline{\mathrm{I}} .89554$ & 50 \\
\hline II & 78130 & 88000 & 12000 & 90130 & 79 III & 89567 & 10433 & 89544 & 49 \\
\hline 12 & 78147 & 88027 & 11973 & 90120 & 79128 & 89593 & 10407 & 89534 & 48 \\
\hline 13 & 78163 & 88053 & 11947 & 90 III & 79144 & 89619 & 10381 & 89524 & 47 \\
\hline 14 & 78180 & 88079 & 11921 & 90101 & 79160 & 89645 & 10 355 & 89514 & 46 \\
\hline 15 & $\overline{\mathbf{1}} .78197$ & $\overline{\mathbf{1}} .88105$ & o.11 895 & $\overline{\mathbf{I}} .9009 \mathrm{I}$ & $\overline{\mathbf{I}} .79176$ & $\overline{\mathrm{I}} .8967 \mathrm{I}$ & 0.10329 & $\overline{\text { I. }} 89504$ & 45 \\
\hline 16 & 78213 & 88 I 3 I & I 1869 & 90082 & 79192 & 89697 & 10303 & 89495 & 44 \\
\hline 17 & 78230 & 88158 & 11842 & $9007^{2}$ & 79208 & 89723 & 10277 & 89485 & 43 \\
\hline 18 & 78246 & 88184 & II 816 & 90063 & 79224 & 89749 & $1025 \mathrm{I}$ & 89475 & 42 \\
\hline 19 & 78263 & 88 210 & I I 790 & 90053 & 79240 & 89775 & 10225 & 89465 & $4^{1}$ \\
\hline 20 & $\overline{\mathrm{I}} .78280$ & $\overline{\mathrm{I}} .88236$ & O. I I 764 & $\overline{\text { I }} .90043$ & $\overline{\mathbf{I}} .79256$ & $\overline{\mathrm{I}} .8980 \mathrm{I}$ & 0.10199 & $\overline{\mathbf{I}} .89455$ & 40 \\
\hline 21 & 78296 & 88262 & II 738 & 90034 & 79272 & 89827 & 10173 & 89445 & 39 \\
\hline 22 & $7^{8} 3^{1} 3$ & 88289 & II 7 II & 90024 & 79288 & 89853 & 10147 & 89435 & $3^{8}$ \\
\hline 23 & 78329 & 88315 & II 685 & 90 or 4 & 79304 & 89879 & IO 121 & 89425 & 37 \\
\hline 24 & 78346 & 8834 I & II 659 & 90005 & 79319 & 89905 & 10095 & 89415 & 36 \\
\hline 25 & $\overline{\mathbf{I}} \cdot 7^{8} 362$ & $\overline{\mathbf{I}} .88367$ & o.1 I 633 & $\overline{\mathbf{I}} .89995$ & $\overline{\mathrm{I}} .79335$ & $\overline{\mathbf{I}} .8993 \mathbf{I}$ & 0.10069 & $\overline{\mathrm{I}} .89405$ & 35 \\
\hline 26 & $7^{8} 379$ & 88393 & I I 607 & 89985 & $7935 \mathrm{I}$ & 89957 & 10043 & 89395 & 34 \\
\hline 27 & 78395 & 88420 & II 580 & 89976 & 79367 & 89983 & 10017 & $8 y 385$ & 33 \\
\hline 28 & $7^{8} 412$ & 88446 & II 554 & 89966 & 79383 & 90009 & 09991 & 89375 & 32 \\
\hline 29 & 78428 & $8847^{2}$ & II 528 & 89956 & 79399 & 90035 & 09965 & 89364 & 31 \\
\hline 30 & $\overline{\mathrm{I}} \cdot 78445$ & $\overline{\mathrm{I}} .88498$ & o. 11502 & $\overline{\mathrm{I}} .89947$ & $\overline{\mathrm{I}} .794 \mathrm{I} 5$ & $\overline{\mathbf{I}} .9006 \mathrm{I}$ & 0.09939 & $\overline{\mathbf{I}} .89354$ & 30 \\
\hline 31 & $7846 i$ & 88524 & II 476 & 89937 & 79431 & 90086 & 09914 & 89344 & 29 \\
\hline 32 & 78478 & $8855^{\circ}$ & II 450 & 89927 & 79447 & 90112 & 09888 & 89334 & 28 \\
\hline 33 & 78494 & 88577 & I I 423 & 89918 & 79463 & 90138 & 09862 & 89324 & 27 \\
\hline 34 & 78510 & 88603 & I I 397 & 89908 & 79478 & 90164 & 09836 & 89314 & 26 \\
\hline 35 & $\overline{\mathrm{I}} .7^{8} 527$ & $\overline{\mathbf{I}} .88629$ & 0.1137 I & $\overline{\mathbf{I}} .89898$ & $\overline{\mathbf{r}} .79494$ & $\overline{\mathbf{I}} .90190$ & 0.09810 & $\overline{\mathbf{I}} .89304$ & 25 \\
\hline 36 & 78543 & 88655 & I I 345 & 89888 & 79510 & 90216 & 09784 & 89294 & 24 \\
\hline 37 & 78560 & $8868 \mathbf{I}$ & 11319 & 89879 & 79526 & 90242 & $0975^{8}$ & 89284 & 23 \\
\hline $3^{8}$ & 78576 & 88707 & II 293 & 89869 & 79542 & 90268 & 09732 & 89274 & 22 \\
\hline 39 & 78592 & 88733 & II 267 & 89859 & $7955^{8}$ & 90294 & 09706 & 89264 & 21 \\
\hline 40 & $\overline{\mathbf{I}} .78609$ & $\overline{\mathbf{I}} .88759$ & O.I I 24 I & $\overline{\mathbf{1}} .89849$ & $\overline{\mathbf{x}} .79573$ & $\overline{\mathbf{1}} .90320$ & 0.09680 & $\overline{\mathrm{x}} .89254$ & 20 \\
\hline $4 I$ & 78625 & 88786 & II 214 & 89840 & 79589 & 90346 & 09654 & 89244 & 19 \\
\hline 42 & 78642 & 88812 & 11188 & 89830 & 79605 & 90371 & 09629 & 89233 & 18 \\
\hline 43 & 78658 & 88838 & II 162 & 89820 & 79621 & 90397 & 09603 & 89223 & 17 \\
\hline 44 & 78674 & 88864 & 11 136 & 89810 & 79636 & 90423 & 09577 & 89213 & I6 \\
\hline 45 & $\overline{\mathbf{I}} \cdot 7869 \mathrm{I}$ & $\overline{\mathbf{I}} .88890$ & 0.11110 & $\overline{\mathrm{I}} .8980 \mathrm{I}$ & $\overline{\mathrm{I}} .79652$ & $\overline{\mathbf{I}} .90449$ & $0.0955^{1}$ & $\overline{\mathbf{1}} .89203$ & 15 \\
\hline 46 & 78707 & 88916 & I 1084 & $8979 \mathrm{I}$ & 79668 & 90475 & 09525 & 89193 & 14 \\
\hline 47 & 78723 & 88942 & $1105^{8}$ & 89781 & 79684 & 90501 & og 499 & 89183 & 13 \\
\hline 48 & 78739 & 88968 & 11032 & 89771 & 79699 & 90527 & 09473 & 89173 & 12 \\
\hline 49 & 78756 & 88994 & 11006 & 89761 & 79715 & 90553 & 09447 & 89162 & II \\
\hline 50 & $\overline{\mathrm{I}} \cdot 78772$ & $\overline{\mathrm{I}} .89020$ & 0. 10980 & $\overline{\mathbf{I}} .8975^{2}$ & $\overline{\mathbf{I}} \cdot 7973 \mathbf{I}$ & $\overline{\mathbf{I}} .90 \quad 578$ & 0.09422 & $\overline{\mathrm{I}} .89 \times 52$ & 10 \\
\hline $5^{1}$ & 78788 & 89046 & 10954 & 89742 & 79746 & 90604 & 09396 & 89142 & 9 \\
\hline $5^{2}$ & 78805 & 89073 & 10927 & 89732 & 79762 & 90630 & 09370 & 89132 & 8 \\
\hline 53 & 78821 & 89099 & 10901 & $89 ? 22$ & 79778 & 90656 & o9 344 & 89122 & 7 \\
\hline 54 & 78837 & 89125 & 10875 & 89712 & 79793 & 90682 & 09318 & 89112 & 6 \\
\hline 55 & $\overline{\mathbf{I}} .78853$ & $\overline{\mathbf{I}} .89151$ & 0.10849 & $\overline{\mathbf{I}} .89702$ & $\overline{\mathbf{I}} .79809$ & $\overline{\mathbf{I}} .90708$ & 0.09292 & $\overline{\mathrm{I}} .89 \mathrm{IOI}$ & 5 \\
\hline $5^{6}$ & 78869 & 89177 & 10823 & 89693 & 79825 & 90734 & 09266 & $8909 \mathrm{I}$ & 4 \\
\hline 57 & 78886 & 89203 & 10797 & 89683 & 79840 & 90759 & $0924 \mathrm{I}$ & 89081 & 3 \\
\hline $5^{8}$ & 78902 & 89229 & $1077 i$ & 89673 & 79856 & 90785 & 09215 & 89071 & 2 \\
\hline & 78918 & 89255 & I0 745 & 89663 & 79872 & $908 \mathrm{I} \mathbf{I}$ & 09189 & 89060 & I \\
\hline \multirow[t]{2}{*}{$60^{\prime}$} & $\overline{\mathrm{I}} .78934$ & $\overline{\mathrm{I}} .8928 \mathrm{I}$ & 0.10719 & $\overline{1} .89653$ & $\overline{\mathbf{I}} .79887$ & $\overline{\mathbf{1}} .90837$ & 0.09163 & $\overline{1} .89050$ & $0^{\prime}$ \\
\hline & $\log \cos$ & $\log \cot$ & $\log \tan$ & $\log \sin$ & $\log \mathrm{cos}$ & $\log \cot$ & $\log \tan$ & $\log \sin$ & I \\
\hline
\end{tabular}




\begin{tabular}{|c|c|c|c|c|c|c|c|c|c|}
\hline ' & $\log \sin$ & $\log \tan$ & $\log \cot$ & $\log c 08$ & $\log \sin$ & $\log \tan$ & $\log \cot$ & $\log \cos$ & \\
\hline $0^{\prime}$ & $\overline{1} .79887$ & $\overline{1} .90837$ & 0.09163 & $\overline{1} .89050$ & T. $80 \mathrm{SO}_{7}$ & $\overline{\mathrm{I}} .9^{2} 3^{8 \mathrm{I}}$ & 0.07619 & T.SS 425 & $60^{\prime}$ \\
\hline I & 79903 & $90 \mathrm{~S}_{3}$ & 09137 & 89040 & 80822 & 92407 & o7 593 & $88+15$ & 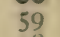 \\
\hline 2 & 79918 & 90889 & 09111 & 89030 & $80 S_{37}$ & 92433 & 07567 & 88404 & $5^{8}$ \\
\hline 3 & 79934 & 90914 & 09086 & 89020 & 80852 & $9^{2} 45^{8}$ & 07542 & 88394 & 57 \\
\hline 4 & 79950 & 90940 & 09060 & 89009 & 80867 & 92484 & 07516 & $88_{3} 83$ & $5^{6}$ \\
\hline 5 & $\overline{1} .79965$ & $\bar{I} .90966$ & 0.09034 & '̄. 88999 & I. $80 \mathrm{SS}_{2}$ & $\overline{1} .92510$ & 0.07490 & $\overline{\mathrm{I}} .8837^{2}$ & 55 \\
\hline 6 & $7998 \mathbf{i}$ & 90992 & 09008 & 88989 & 80897 & 92535 & 07465 & 88362 & 54 \\
\hline 8 & 79996 & 91018 & 08982 & 88978 & 80912 & $9^{2} 561$ & 07439 & 88351 & 53 \\
\hline & 80012 & 91043 & 08957 & 88968 & 80927 & $925^{87}$ & 07413 & 88340 & 52 \\
\hline 9 & 80027 & 91009 & or 931 & 88958 & 80942 & 12 & 07 & 88330 & 51 \\
\hline 10 & 1. 80043 & $\overline{1} .91095$ & 0.08905 & 1. 88948 & I. 80957 & 1.92 $63^{8}$ & 0.07362 & $\overline{1} .88319$ & 50 \\
\hline 12 & $\begin{array}{l}80058 \\
80074\end{array}$ & 91121 & 08879 & $\begin{array}{l}88937 \\
88027\end{array}$ & $\begin{array}{l}80972 \\
80987\end{array}$ & $\begin{array}{l}92663 \\
92680\end{array}$ & $\begin{array}{l}07337 \\
07311\end{array}$ & $\begin{array}{l}8830 S \\
88309\end{array}$ & $\begin{array}{l}49 \\
48\end{array}$ \\
\hline 13 & 80089 & 91172 & 08828 & 88917 & 81002 & 92715 & 07285 & 88287 & 47 \\
\hline 14 & 80105 & 91198 & 08802 & 88906 & 81017 & 92740 & 07260 & 88276 & 46 \\
\hline 15 & $\overline{\mathrm{I}} .80120$ & $\overline{1} .91224$ & 0.08776 & $\overline{\mathbf{I}} .88896$ & T. 81032 & $\overline{\mathbf{1}} .92766$ & 0.07234 & $\overline{\mathrm{I}} .89266$ & 45 \\
\hline 16 & 80136 & 91250 & 08750 & 88886 & 81047 & 92792 & 07208 & 88255 & 44 \\
\hline 17 & 80151 & 91276 & $087^{24}$ & 88875 & $8106 I$ & 92817 & 07183 & 88244 & 43 \\
\hline 18 & 80166 & 91301 & o8 699 & 88865 & 81076 & 92843 & 07157 & 88234 & 42 \\
\hline 19 & 80182 & 91327 & 08673 & 88855 & 81091 & 92868 & 07132 & 88223 & $4^{I}$ \\
\hline 20 & $\bar{I} .80197$ & I. 91353 & 0.08647 & Т. 88844 & $\overline{\mathbf{I}} .8 \mathrm{I} 106$ & I. 92894 & 0.07106 & $\overline{1} .88212$ & 40 \\
\hline 21 & 80213 & 91379 & $0862 \mathrm{I}$ & 88834 & 81121 & 92920 & 07080 & OI & 39 \\
\hline 22 & 80228 & 91404 & 08596 & 88824 & 81136 & 92945 & 07055 & 91 & $3^{8}$ \\
\hline 23 & 80244 & 91430 & 08570 & 88813 & $8115 I$ & 92971 & 07029 & 88180 & 37 \\
\hline 24 & 80259 & 91456 & 08544 & $8880_{3}$ & 81166 & 92996 & 07004 & 88169 & 36 \\
\hline 25 & T. 80274 & I. 91482 & 0.08518 & $\overline{\mathbf{I}} .88793$ & $\overline{1} .81$ 1 80 & $\overline{\mathbf{I}} .93022$ & $0.0697^{8}$ & '. $8815^{8}$ & 35 \\
\hline 26 & 80290 & 91507 & 08493 & 88782 & 81195 & 93048 & 06952 & 88148 & 34 \\
\hline 27 & 80305 & 91533 & 08467 & 88772 & 81210 & 93073 & 06927 & 88137 & 33 \\
\hline 28 & 80320 & 91559 & $0844 i$ & 88761 & 81225 & 93099 & 06901 & 88126 & 32 \\
\hline 29 & 80336 & 91585 & 08415 & 88751 & 81240 & 93124 & 06876 & $88 \div 5$ & $3^{1}$ \\
\hline 30 & $\overline{\mathrm{I}} .8035 \mathrm{I}$ & $\bar{I} .91610$ & 0.08390 & $\overline{\mathrm{I}} .88 \mathrm{74I}$ & $\overline{1} .81254$ & $\overline{\mathbf{I}} .93150$ & 0.06850 & I. 88105 & 30 \\
\hline $3^{1}$ & 80366 & 91636 & 08364 & 88730 & 81269 & 93175 & 06825 & 94 & 29 \\
\hline 32 & $80_{3} 82$ & 91662 & $0833^{8}$ & 88720 & 81284 & 93201 & 06799 & 88083 & 28 \\
\hline 33 & 80397 & 91688 & 08312 & 88709 & 81299 & 93227 & 06773 & $880^{2}$ & 27 \\
\hline 34 & 80412 & 91713 & 08287 & 88699 & 81314 & 93252 & 06748 & 88061 & 26 \\
\hline 35 & i. 80428 & $\overline{\mathbf{I}} .91739$ & $0.0826 i$ & $\overline{\mathbf{I}} .88688$ & I. 81328 & $\overline{\mathbf{I}} .93278$ & $0.067^{22}$ & $\overline{\mathbf{1}} .88$ O5 I & 25 \\
\hline 36 & 80443 & 91765 & 08235 & 88678 & 81343 & 93303 & 06697 & 88040 & 24 \\
\hline 37 & $8045^{8}$ & 91791 & 08209 & 88668 & $8135^{8}$ & 93329 & 06671 & 88029 & 23 \\
\hline $3^{8}$ & 80473 & 91816 & 08184 & 88657 & 81372 & 93354 & 06646 & 88018 & 22 \\
\hline 39 & 80489 & 91842 & $15^{8}$ & 47 & 81387 & 93380 & 6620 & 07 & 21 \\
\hline 40 & $\overline{1} .80504$ & $\overline{\mathrm{z}} .91868$ & 0.08132 & $\overline{7} .88636$ & $\overline{\mathrm{I}} .81402$ & $\overline{1} .93406$ & 0.06594 & Т.87996 & 20 \\
\hline 41 & 80519 & 91893 & 08107 & 26 & 81417 & $9343^{1}$ & 06569 & $8799_{5}$ & 19 \\
\hline 42 & 80534 & 91919 & $0808 i$ & 88615 & $8143 I$ & 93 & 06543 & 87975 & 18 \\
\hline 43 & 80550 & 91945 & 08055 & 88605 & 81446 & 93482 & 06518 & 87964 & 17 \\
\hline 44 & 80565 & 91971 & 8029 & 88594 & $146 r$ & 93508 & 06492 & 87953 & 16 \\
\hline 45 & 1. $805^{80}$ & $\overline{\text { I. }} 91996$ & 0.08004 & $\overline{\mathbf{x}} .88584$ & $\overline{\mathrm{I}} .81475$ & $\overline{1} .93533$ & 0.06467 & $\overline{1} .87942$ & 15 \\
\hline 46 & 80595 & 92022 & 07978 & 88573 & 81490 & 93 & $0644 i$ & 87931 & 14 \\
\hline 47 & 80610 & 92048 & 07952 & 88563 & 81505 & 93584 & 06416 & 87920 & 13 \\
\hline 48 & 80625 & 92073 & 07927 & 88552 & 81519 & 93610 & 06390 & 87 & 12 \\
\hline 49 & $8064 i$ & 92099 & 07901 & 88542 & 81534 & 93636 & 06364 & 87898 & II \\
\hline 50 & $\overline{1} .80656$ & $\overline{1} .92125$ & 0.07875 & $\overline{8} .88531$ & 1. 81549 & $\overline{1} .93661$ & 0.06339 & $\overline{7} .87887$ & 10 \\
\hline $5^{2}$ & 80671 & 92150 & 07850 & 88521 & 81563 & 93687 & 06313 & 87977 & 3 \\
\hline 52 & 80686 & 92176 & 07824 & 88510 & $8157^{8}$ & 93712 & 06288 & 87566 & 8 \\
\hline 53 & 80701 & 92202 & $0779^{8}$ & 88499 & 81592 & $9373^{8}$ & 06262 & 87855 & 7 \\
\hline 54 & 80716 & 92227 & 07773 & 88489 & 81607 & 93763 & 06237 & 87844 & 6 \\
\hline 55 & T. So $73 \mathrm{I}$ & $\overline{1} .92253$ & 0.07747 & $\bar{x} .8847^{8}$ & $\overline{1} .81622$ & ז. $937^{89}$ & 0.06211 & 1.87833 & 5 \\
\hline 5 & 80746 & 92279 & $077^{21}$ & 88468 & 81636 & 93814 & 06156 & $87 \$ 22$ & 4 \\
\hline 57 & 80762 & 92304 & 07696 & 88457 & 81651 & 93840 & 06160 & $87 \sin$ & 3 \\
\hline $5^{8}$ & 80777 & 92330 & 07670 & 88447 & 81665 & 93865 & of 135 & 87800 & 2 \\
\hline 59 & 80792 & 92356 & 09644 & $8843^{6}$ & 81680 & $93 \mathrm{Sol}$ & 06109 & $877^{819}$ & 8 \\
\hline \multirow[t]{2}{*}{$60^{\prime}$} & I. $80 \mathrm{SO}_{7}$ & 1.02381 & 0.07619 & 7.88425 & I.81604 & 1.03016 & 0.06084 & $7.8777^{8}$ & $0^{\prime}$ \\
\hline & $\log 008$ & $\log \cot$ & $\log \tan$ & $\log _{\sin }$ & $\log 008$ & $\log \cot$ & $\log \tan$ & $\log _{\sin }$ & \\
\hline
\end{tabular}




\begin{tabular}{|c|c|c|c|c|c|c|c|c|c|}
\hline ! & $\log \sin$ & $\log \tan$ & $\log \cot$ & $\log \cos$ & $\log \sin$ & $\log \tan$ & $\log \cot$ & $\log 008$ & \\
\hline $0^{\prime}$ & I.81694 & $\overline{1} .93916$ & $0.0608_{4}$ & $\overline{1} .8777^{8}$ & I. $8255^{1}$ & $\overline{\mathbf{I}} .95444$ & $0.0455^{6}$ & $\overline{1} .87107$ & $60^{\prime}$ \\
\hline I & 81709 & 93942 & 06058 & 87767 & 82565 & 95469 & 04531 & 87096 & 59 \\
\hline 2 & 81723 & 93967 & 06033 & 87756 & 82579 & 95495 & 04505 & 87085 & 58 \\
\hline 3 & $8173^{8}$ & 93993 & 06007 & 87745 & 82593 & 95520 & 04480 & 87073 & 57 \\
\hline 4 & $8175^{2}$ & 94018 & 05982 & 87734 & 82607 & 95545 & 04455 & 87062 & $5^{6}$ \\
\hline 5 & $\overline{\mathbf{I}} .81767$ & $\overline{\mathbf{I}} .94044$ & 0.05956 & $\overline{1} .87723$ & $\overline{1} .82621$ & $\bar{I} .9557$ I & 0.04429 & $\overline{\mathbf{1}} .87050$ & 55 \\
\hline 6 & $8178 I$ & 94069 & O5 93I & 87712 & 82635 & 95596 & 04404 & 87039 & 54 \\
\hline 7 & 81796 & 94095 & 05905 & 87701 & 82649 & 95622 & $0437^{8}$ & 87028 & 53 \\
\hline 8 & 8I Sio & $9+120$ & o5 880 & 87690 & 82663 & 95647 & 04353 & 87016 & $5^{2}$ \\
\hline 9 & 81825 & 94146 & 05854 & 87679 & 82677 & 95672 & 04328 & 87005 & $5^{1}$ \\
\hline 10 & $\overline{\mathbf{I}} .81839$ & $\bar{I} .94$ I I I & 0.05829 & $\overline{\mathbf{I}} .87668$ & $\overline{\mathbf{I}} .82691$ & $\overline{\mathbf{I}} .95698$ & 0.04302 & $\overline{\mathbf{x}} .86993$ & 50 \\
\hline II & $81 S_{54}$ & 94197 & 05803 & 87657 & 82705 & 95723 & 04277 & 86982 & 49 \\
\hline 12 & 81868 & 94222 & 05778 & 87646 & 82719 & 95748 & 04252 & 86970 & 48 \\
\hline 13 & 81852 & 94248 & $0575^{2}$ & 87635 & 82733 & 95774 & 04226 & 86959 & 47 \\
\hline 14 & 81897 & 94273 & O5 727 & 87624 & 82747 & 95799 & 04201 & 86947 & 46 \\
\hline 15 & $\bar{I} .819 \mathrm{II}$ & $\bar{I} .94299$ & 0.05701 & $\overline{1} .87613$ & $\overline{\mathbf{I}} .8276 \mathrm{I}$ & $\overline{\mathbf{I}} .95825$ & 0.04175 & $\overline{\mathbf{I}} .86936$ & 45 \\
\hline 16 & 81926 & 94324 & .05676 & 87601 & 82775 & 95850 & 04 I 50 & 86924 & 44 \\
\hline 17 & 81940 & $9435^{\circ}$ & 05650 & 87590 & 82788 & 95875 & 04125 & 86913 & 43 \\
\hline 18 & 81955 & 94375 & 05625 & 87579 & 82802 & 95901 & 04099 & 86902 & 42 \\
\hline 19 & 81969 & 94401 & 05 599 & 87568 & 82816 & 95926 & 04074 & 86890 & $4 I$ \\
\hline 20 & $\overline{\mathbf{I}} .81983$ & $\bar{I} \cdot 94426$ & 0.05574 & $\overline{\mathbf{I}} .87557$ & $\overline{\mathbf{I}} .82830$ & $\overline{\mathbf{I}} .95952$ & 0.04048 & $\overline{\mathbf{I}} .86879$ & 40 \\
\hline 21 & 81998 & $9445^{2}$ & O5 54.8 & 87546 & 82844 & 95977 & 04023 & 86867 & 39 \\
\hline 22 & 82012 & 94477 & 05523 & 87535 & 82858 & 96002 & 03998 & 86855 & $3^{8}$ \\
\hline 23 & 82026 & 94503 & 05497 & 87524 & 82872 & 96028 & 03972 & 44 & 37 \\
\hline 24 & 82041 & 94528 & 05472 & 87513 & 82885 & - 96053 & O3947 & 32 & 36 \\
\hline 25 & $\overline{\mathbf{I}} .82055$ & $\overline{\mathbf{I}} .94554$ & 0.05446 & $\overline{\mathbf{I}} .8750 \mathrm{I}$ & $\overline{\mathbf{I}} .82899$ & $\overline{\mathbf{I}} .96078$ & 0.03922 & $\overline{\mathrm{I}} .8682 \mathrm{I}$ & 35 \\
\hline 26 & 82069 & 94579 & 05421 & 87490 & 82913 & 96104 & o3 896 & 86809 & 34 \\
\hline 27 & 82084 & 94604 & 05396 & 87479 & 82927 & 96 I 29 & O3 87I & 86798 & 33 \\
\hline 28 & 82098 & 94630 & 05370 & 87468 & 82941 & 96 I 55 & 03845 & 86 & 32 \\
\hline 29 & 82 II 2 & 94655 & o5 345 & 87457 & 82955 & 80 & 03820 & 775 & $3 I$ \\
\hline 30 & $\overline{1} .82$ I 26 & $\overline{\mathrm{I}} .9468 \mathrm{I}$ & 0.05319 & $\overline{\mathrm{I}} .87446$ & $\overline{\mathrm{I}} .82968$ & $\overline{\mathbf{I}} .96205$ & 0.03 & .86763 & 30 \\
\hline 31 & 82141 & 94706 & 05294 & 87434 & 82982 & $23 I$ & 03769 & $75^{2}$ & 29 \\
\hline 32 & $82 \times 55$ & 94732 & 05268 & 87423 & 96 & 96256 & 03744 & 86740 & 28 \\
\hline 33 & 82169 & 94757 & O5 243 & 87412 & 83 oro & $9628 \mathrm{I}$ & 03719 & 86728 & 27 \\
\hline 34 & 82184 & 94783 & 05217 & 87401 & 83023 & 96307 & 03693 & 86717 & 26 \\
\hline 35 & $\overline{1} .82198$ & $\overline{\mathbf{I}} .94808$ & 0.05192 & $\overline{\mathbf{I}} .87390$ & $\bar{x} 83037$ & $\bar{x} .96332$ & 0.03668 & $\overline{\mathbf{I}} .86705$ & 25 \\
\hline 36 & 82212 & 94834 & o5 I 66 & 87378 & $83 C_{5} I$ & 96357 & 03643 & 86694 & 24 \\
\hline 37 & 82226 & 94859 & 05 I II & 87367 & 83065 & 96383 & 03617 & 86682 & 23 \\
\hline $3^{8}$ & 82240 & 94884 & 05116 & 87356 & 83078 & 96408 & 03592 & 86670 & 22 \\
\hline 39 & 82255 & 94910 & 05090 & 87345 & 83092 & 96433 & 03567 & 59 & 21 \\
\hline 40 & $\overline{\mathrm{I}} .82269$ & $\overline{\mathbf{I}} .94935$ & 0.05065 & $\overline{\mathbf{I}} .87 .334$ & $\overline{\mathrm{I}} .83106$ & $\bar{I} .96$ & 0.03 & 47 & 20 \\
\hline $4 I$ & $82 \quad 283$ & $9496 \mathrm{I}$ & 05039 & 87322 & 83 I 20 & 96484 & 03516 & 535 & 19 \\
\hline 42 & 82297 & 94986 & 05 or 4 & 873 II & 83 I 33 & 96510 & 03490 & 86624 & 18 \\
\hline 43 & 82311 & 95 OI 2 & 04988 & 87300 & 83147 & 96535 & 03465 & 86612 & 17 \\
\hline 44 & 82326 & 95037 & 04963 & 87288 & $8316 I$ & 96560 & 03440 & 100 & 16 \\
\hline 45 & $\overline{\mathbf{1}} .82340$ & $\overline{1} .95062$ & 0.04938 & $\overline{\mathrm{I}} .87277$ & $\overline{\mathrm{I}} .83 \mathrm{I} 74$ & $\overline{\mathbf{I}} .96586$ & 0.03414 & $\overline{\mathbf{I}} .86589$ & 15 \\
\hline 46 & 82354 & 95088 & 04912 & 87266 & 83188 & 966 I I & O3 389 & 577 & 14 \\
\hline 47 & 82368 & $95 \simeq 13$ & 04887 & 87255 & 83202 & 96636 & 03364 & 86565 & 13 \\
\hline 48 & $823^{82}$ & 95139 & $0486 i$ & 87243 & 83215 & 96662 & O3 $33^{8}$ & 86554 & 12 \\
\hline 49 & 82396 & 95 I 64 & 04836 & 87232 & 83229 & 96687 & O3 3 I 3 & 86542 & I I \\
\hline 50 & $\overline{1} .82410$ & $\overline{\mathrm{I}} .95190$ & 0.04810 & $\overline{\mathrm{I}} .8722 \mathrm{I}$ & $\overline{\mathrm{I}} .83242$ & $\overline{\mathbf{I}} .96712$ & 0.03288 & $\overline{\mathbf{1}} .86$ & 10 \\
\hline $5^{1}$ & 82424 & 95215 & 04785 & 87209 & 83256 & 96738 & 03262 & 86518 & 9 \\
\hline $5^{2}$ & 82439 & 95240 & 04760 & 87198 & 83270 & 96763 & O3 237 & 86507 & 8 \\
\hline 53 & 82453 & 95266 & 04734 & 87187 & 83283 & 96788 & 03212 & 495 & 7 \\
\hline 54 & 82467 & 95291 & 04709 & 87175 & 83297 & 96814 & 03186 & 86483 & 6 \\
\hline 55 & $\overline{1} .82481$ & $\overline{1} .95317$ & 0.04683 & $\overline{\mathrm{I}} .87 \mathrm{I} 64$ & $\overline{\mathbf{I}} .83310$ & $\overline{\mathbf{I}} .96839$ & $0.0316 \mathrm{I}$ & $\overline{1} .8647^{2}$ & 5 \\
\hline 56 & 82495 & 95342 & 04658 & 87153 & 83324 & 96864 & 03136 & 86463 & 4 \\
\hline 57 & 82509 & 95368 & 04632 & 87 I 41 & $8333^{8}$ & 96890 & O3 IIO & 86448 & 3 \\
\hline 58 & 82523 & 95393 & 04607 & 87 130 & $8335 \mathrm{I}$ & 96915 & 03085 & 86436 & 2 \\
\hline \multirow{3}{*}{$60^{\prime}$} & 82537 & 95418 & 04582 & 87 I 19 & 83365 & 96940 & 03060 & 86425 & I \\
\hline & $\overline{1} .82551$ & $\bar{x} .95444$ & $0.0455^{6}$ & $\overline{\mathbf{I}} .87 \quad 107$ & I. 83378 & $\overline{1} .96966$ & 0.03034 & $\overline{\mathrm{I}} .864 \mathrm{I} 3$ & $0^{\prime}$ \\
\hline & $\log \cos$ & $\log \cot$ & $\log \tan$ & $\log \sin$ & $\log \cos$ & $\log \cot$ & $\log \tan$ & $\log \sin$ & 1 \\
\hline
\end{tabular}




\begin{tabular}{|c|c|c|c|c|c|c|c|c|c|}
\hline , & $\log \sin$ & $\log \tan$ & $\log \cot$ & $\log 608$ & $\log \sin$ & $\log \tan$ & $\log \cot$ & $\log \cos$ & \\
\hline$\overline{0^{\prime}}$ & $\overline{\mathbf{1}} .8_{3} 37^{8}$ & $\overline{1} .96966$ & 0.03034 & $\overline{1} .86413$ & $\overline{1} .84177$ & 1.98484 & 0.01516 & $\overline{1} . S_{5} 693$ & $60^{\prime}$ \\
\hline 1 & 83392 & 96991 & 03009 & $86+01$ & $8+190$ & 98509 & O1 491 & 85651 & 59 \\
\hline 2 & 83405 & 97016 & 02984 & $56{ }_{3} 89$ & $842 \mathrm{O}_{3}$ & 95534 & ol 466 & 85669 & 58 \\
\hline 3 & 83419 & 97042 & $0295^{\circ}$ & 86377 & 84216 & 43560 & or 440 & $8_{5} 657$ & 57 \\
\hline 4 & $83+32$ & 97067 & 02933 & 86306 & 84229 & $-9 S 5 S_{5}$ & OI 415 & 85645 & 56 \\
\hline 5 & ก. 83446 & T. 97092 & 0.02908 & i. .56354 & $\overrightarrow{1} .8+24^{2}$ & 1. 99610 & 0.01390 & $\overline{1} .8_{5} 6 j^{2}$ & 55 \\
\hline 6 & 83459 & 97118 & 02582 & 86342 & 84255 & 98635 & or 365 & 85620 & 54 \\
\hline 7 & $83+73$ & 97143 & 02857 & 86330 & 84269 & $9860 \mathrm{I}$ & ol 339 & 85608 & 53 \\
\hline 8 & 83486 & 97168 & $0283^{2}$ & 86315 & 84282 & $9 \$ 686$ & o1 314 & 85596 & 52 \\
\hline 9 & 83500 & 97193 & $02 \mathrm{SO}_{7}$ & 86306 & 84295 & 98711 & Ol 289 & $8_{5} 5^{8} 3$ & 51 \\
\hline 10 & $\overline{1} . S_{3513}$ & $\overline{\mathbf{I}} .97219$ & $0.0278 i$ & ㄱ. 86295 & $\overline{1} .84308$ & $\overline{\mathbf{I}} .9 \$ 737$ & 0.01263 & $\overline{\mathrm{I}} .8557^{1}$ & 50 \\
\hline 11 & 83527 & $972+4$ & 02756 & 86283 & $8_{4321}$ & 98762 & $0123 \mathrm{~S}$ & 85559 & 49 \\
\hline 12 & 83540 & 97269 & 02731 & 86271 & 84334 & $98 \div 57$ & Or 213 & 85547 & $4^{5}$ \\
\hline 13 & 83554 & 97295 & 02705 & 86259 & 84347 & 98812 & 01188 & 85534 & 47 \\
\hline 14 & 83567 & 97320 & 02680 & 86247 & 84360 & $9 \mathrm{~S} \mathrm{~S}_{3} \mathrm{~S}$ & ol 162 & $8_{5522}$ & 46 \\
\hline 15 & $\overline{\mathbf{I}} .8_{3} 5^{8 \mathrm{r}}$ & $\overline{\mathrm{I}} .97345$ & 0.02655 & โ. $.86 ́ 235$ & 1.84373 & $\overline{1} .9 \mathrm{~S}_{3} 6_{3}$ & 0.01137 & $\overline{1} .85510$ & 45 \\
\hline 16 & 83594 & $9737 \mathrm{t}$ & 02629 & 86223 & 84385 & 95888 & 01112 & 85497 & 44 \\
\hline 17 & 8360 & 97396 & 02604 & 86211 & $8+398$ & 98913 & ol oSy & 85485 & 43 \\
\hline 18 & 83621 & 97421 & 02579 & 86200 & 84411 & $9 \$ 939$ & ol 061 & 85473 & 42 \\
\hline 19 & 83634 & 97447 & 02553 & 86 ISS & 84424 & 98 y6 4 & 01036 & 85460 & 41 \\
\hline 20 & I. $S_{3} 64 \mathrm{~S}$ & $\overline{\mathbf{I}} .97472$ & 0.02528 & $\overline{1} .86176$ & $\overline{1} .84437$ & ז.98 989 & 0.01011 & I. $S_{544} \mathrm{~S}$ & 40 \\
\hline 21 & 83661 & 97497 & $\mathrm{O}_{2} \mathrm{5O}_{3}$ & 86164 & $8+450$ & 99015 & $\infty 98_{5}$ & 85436 & 30 \\
\hline 22 & 83674 & 97523 & 02477 & 86152 & 84463 & 99040 & 00960 & 85423 & 38 \\
\hline 23 & 83688 & $9754^{8}$ & $0245^{2}$ & 86140 & 84476 & 99065 & 00935 & 85411 & 37 \\
\hline 24 & 83701 & 97573 & 02427 & 86128 & 84489 & 99090 & $\infty 0910$ & 85399 & 36 \\
\hline 25 & ㄱ. $\delta_{3} 715$ & $\overline{1} .97598$ & 0.02402 & $\overline{1} .86116$ & I. 84502 & $\overline{1} .99116$ & 0.00884 & $\overline{1} .85,386$ & 35 \\
\hline 26 & 83728 & 97624 & $0237^{6}$ & 86104 & 84515 & 99141 & 00859 & 85374 & 34 \\
\hline 27 & 83741 & 97649 & 02351 & 86092 & 84528 & $99 \times 66$ & $00 \$_{34}$ & 85361 & 33 \\
\hline 28 & 83755 & 97674 & 02326 & 86030 & 84540 & 99191 & $\infty 809$ & 85349 & 32 \\
\hline 29 & 83768 & 97700 & 02300 & 86068 & 84553 & 99217 & $\infty 7_{7} 8$ & 85337 & 31 \\
\hline 30 & ‥ 8378 I & $\overline{\mathbf{I}} .97725$ & 0.02275 & т. 86056 & I. 84566 & 1. .99242 & 0.00758 & $\overline{1} .85324$ & 30 \\
\hline 31 & 83795 & 97750 & 02250 & $860+4$ & 84579 & 99267 & $\infty 0733$ & 85312 & 29 \\
\hline 32 & 83508 & $9777^{6}$ & 02224 & $860_{32}^{2}$ & 84592 & 99293 & 00707 & 85299 & $2 S$ \\
\hline 33 & $8_{3} 8_{21}$ & $97 \mathrm{Sol}$ & 02199 & 86020 & 84605 & 99318 & 00682 & 85287 & 27 \\
\hline 34 & $8_{3} 8_{34}$ & 97826 & 02174 & 86008 & 84618 & 99343 & $\infty 0657$ & 85274 & 26 \\
\hline 35 & T. $s_{3} 8_{4} S^{3}$ & $\bar{I} .978_{51}$ & 0.02149 & $\overline{1} .85996$ & $\overline{1} .84630$ & Т. 99368 & 0.00632 & $\overline{1} .85262$ & 25 \\
\hline 30 & $83 \operatorname{sot}$ & $97 \$ 77$ & 02123 & 85984 & 84643 & 99394 & oo 606 & 85250 & 24 \\
\hline 37. & $8_{3} 84$ & 97902 & $0209 \$$ & $8597^{2}$ & 84656 & 99419 & $\infty 5_{5} \mathbf{1}$ & 85237 & 23 \\
\hline $3^{8}$ & 8385 & 97927 & 02073 & 85060 & $8466 n$ & 99444 & 00556 & 85225 & 22 \\
\hline 39 & $8390 i$ & 97953 & $0204 T$ & 85948 & 84682 & 99469 & $\infty 0031$ & $s_{5} 212$ & 21 \\
\hline 40 & 7.83914 & '. 97978 & 0.02022 & T. 85936 & 7.84694 & $\overline{1} .99495$ & 0.00505 & T. $\$_{5} 200$ & 20 \\
\hline 41 & 83927 & 98003 & 01997 & 85924 & 84707 & 99520 & 00450 & 85187 & 11) \\
\hline 42 & 83940 & 95029 & 01971 & $S_{5012}$ & 84720 & 99545 & 00455 & $S_{5} 175$ & 18 \\
\hline 43 & 83954 & 98054 & o1 946 & 85,00 & 84733 & 99570 & 00430 & 85162 & 17 \\
\hline 44 & 83067 & 98079 & 01921 & $85 \mathrm{sss}$ & 84745 & 99596 & 00404 & S5 150 & 16 \\
\hline 45 & T.83980 & T.9.8 104 & 0.01896 & ․ 85876 & I. $8475^{8}$ & 1. .90621 & 0.00379 & I. 85137 & 15 \\
\hline 46 & 83993 & 98130 & 01870 & $85 \mathrm{~S}_{4}$ & 84771 & 99646 & $\infty \quad 354$ & 85125 & 14 \\
\hline 47 & 84006 & 98155 & ol $\mathrm{S}_{45}$ & 85851 & 84784 & 99672 & 00,328 & 85112 & 13 \\
\hline 48 & 84020 & 98180 & ol 820 & 85839 & 84796 & $99(x) 7$ & 00303 & 85100 & 12 \\
\hline 49 & 84033 & 98206 & 01794 & 85827 & 84809 & 99722 & 00275 & $8_{5}$ os & 11 \\
\hline 50 & $\overline{7} .84046$ & $\bar{T} .98231$ & $0.017^{6}(x)$ & $\overline{1} .85815$ & $\overline{1} .8_{4} S_{22}$ & 7.99747 & 0.00253 & i.s5 074 & 10 \\
\hline 51 & 84059 & 98256 & or 744 & $\mathrm{~S}_{5} \mathrm{SO}_{3}$ & $8_{4} s_{35}$ & 90773 & 00227 & 85002 & 0 \\
\hline 52 & 84072 & 95251 & 01710 & 85791 & 84547 & 99798 & $\mathrm{CO}_{202}$ & 85049 & s \\
\hline 53 & 84085 & 95307 & or 693 & $S_{5} 779$ & $S_{4}$ Siro & 99823 & 00177 & 85037 & 7 \\
\hline 54 & 84 ons & 45 332 & 01668 & 857,6 & $8_{4} S_{73}$ & 9984 & Co 152 & 85024 & 6 \\
\hline 55 & I. 44112 & T. .98 .357 & 0.01643 & T. $\S_{5} 754$ & T. $\mathrm{S}_{4} \mathrm{SS}_{5}$ & T.99874 & 0.00126 & T.S5012 & 5 \\
\hline 56 & 84125 & gs $33_{3}$ & 01617 & 85742 & 8485 & $99 \operatorname{sing}$ & no 101 & $s_{4} 99$ & 4 \\
\hline 57 & 8,$1 ; 5$ & $9^{8} 408$ & $015 \% 2$ & 85730 & S4011 & 99924 & 00076 & $\mathrm{~S}_{4} 9 \mathrm{S6}$ & 3 \\
\hline $5^{8}$ & 84151 & is 433 & or 567 & $8_{5}$ 7is & 84923 & 90940 & 00051 & 84274 & 2 \\
\hline $5 n$ & 84164 & $95_{45}$ & or 542 & 85706 & 84936 & i. 09975 & 00025 & $8406 i$ & 1 \\
\hline \multirow[t]{2}{*}{$60^{\prime}$} & I. $S_{4} 177$ & $3.12 \times 454$ & 0.01516 & 183603 & T.8.4 949 & 000000 & 0.00000 & I. $\mathrm{S}_{4} 949$ & $0^{\prime}$ \\
\hline & $\log _{\mathrm{COB}}$ & $\log 60 t$ & $\log \tan$ & $\log$ kin $i$ & $\log 6 \overline{c o s}$ & $\log \mathrm{cent}$ & $\log$ taan & $\log _{\sin }$ & ' \\
\hline
\end{tabular}




\section{CONSTANTS.}

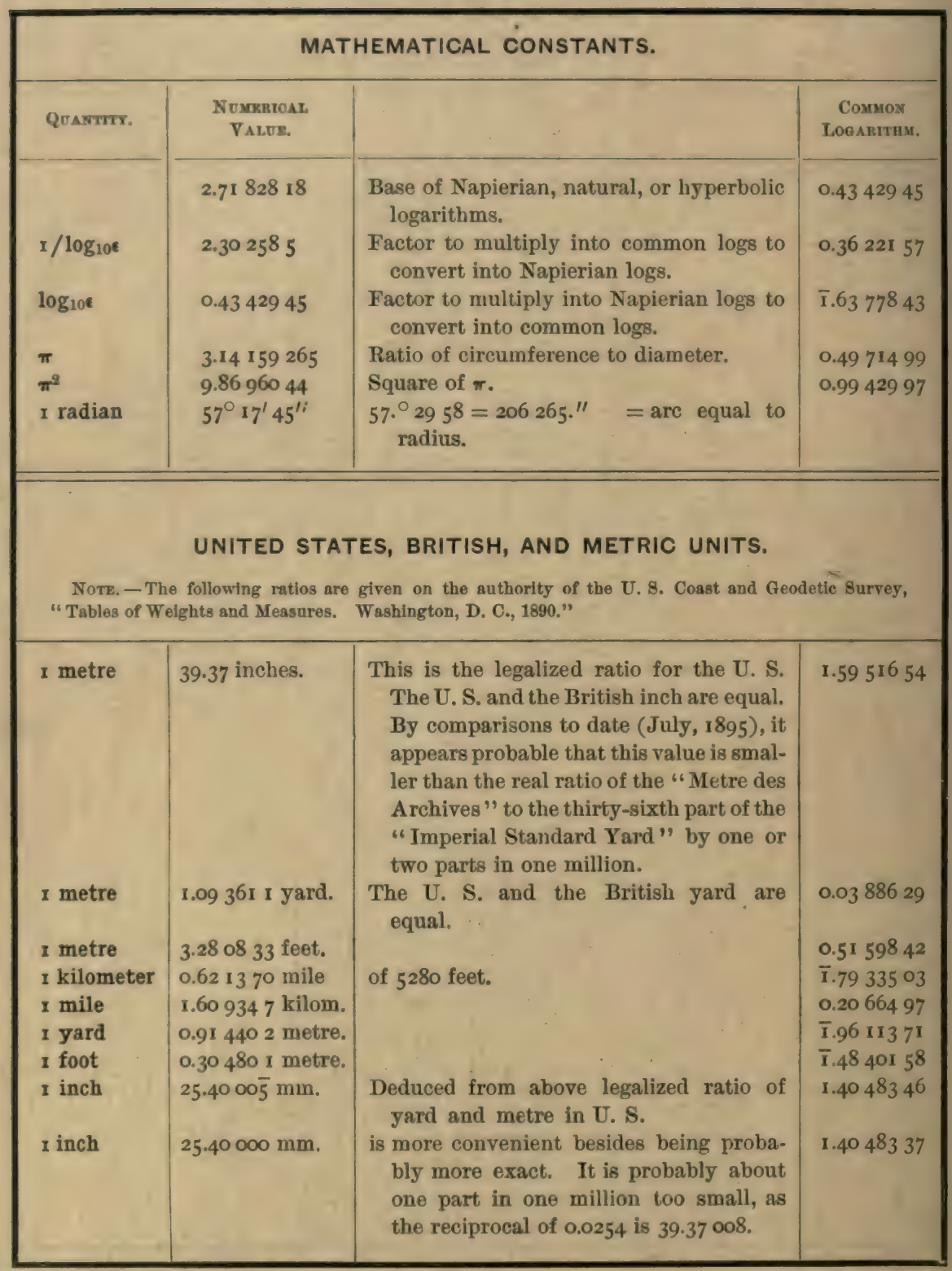




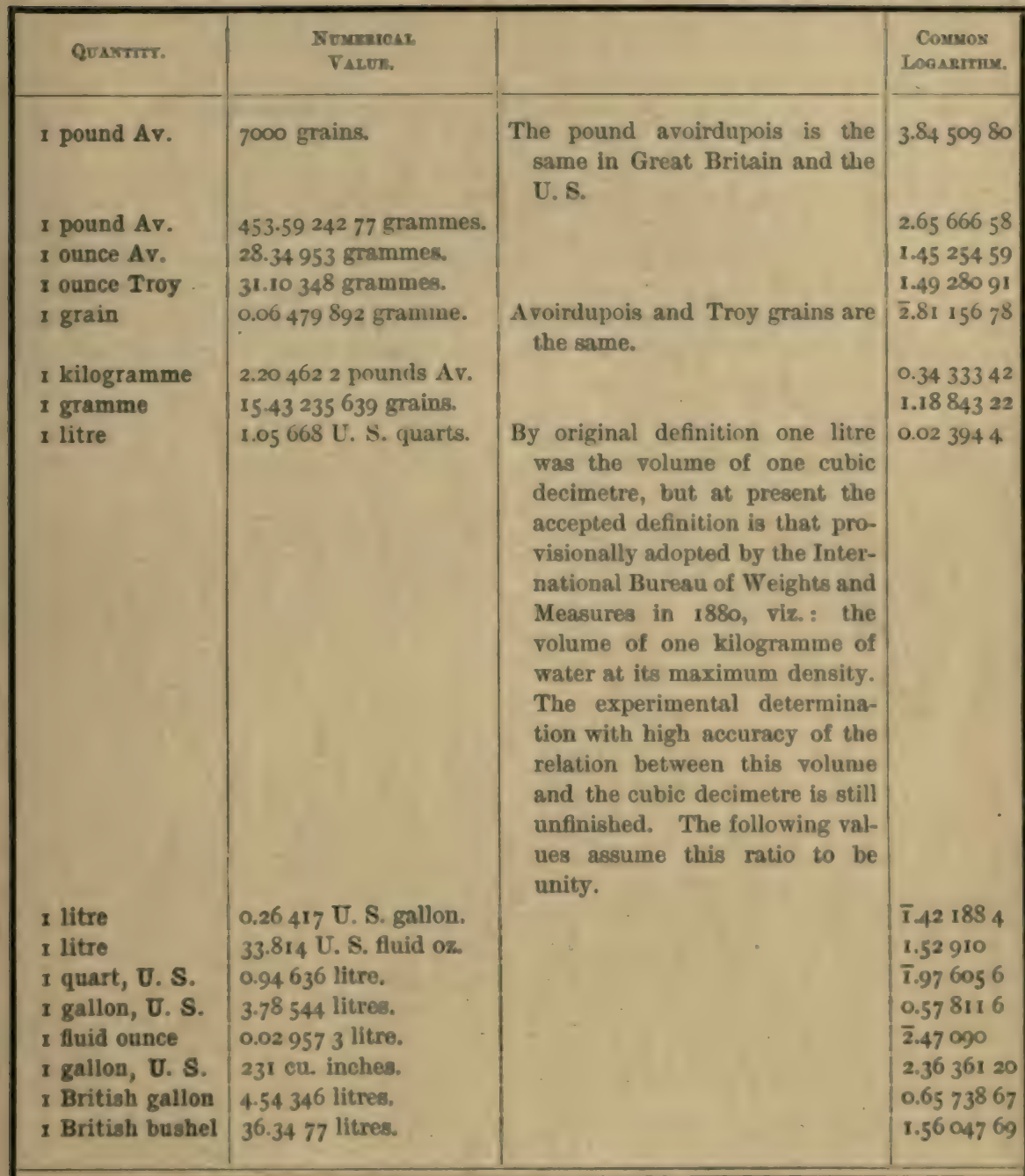

\section{MECHANICAL OR DYNAMICAL EQUIVALENT OF HEAT.}

The best values of this quantity (usually denoted $h y, J$ ) at present attainable (Novemher, 18y5) are the following. The values are uncertain by only about $\&$ one twentieth of one per cent.

427.3 kilogrammetres of work or energy are reyuired at latitule $45^{\circ}$, sea-level $(g=9$ iso.6 e.g.s. $)$, to raise i kilogramme of water through i $\mathrm{C}^{\circ}$ at $15^{\circ} \mathrm{C}$.

$778.8 \mathrm{ft}$. lbs. of work or energy are required at latitule $45 \%$, sea-level $(g=980.6 \mathrm{C.g.8}$.), to raise I $1 \mathrm{~b}$. of water through $1^{\circ}$ Falar. at $59^{\circ}$ Fahr. $\left(=15^{\circ} \mathrm{C}\right.$. $)$. For most engineering purposes $779 \mathrm{ft}$. Ibs. would be near enough. 
$1402 \mathrm{ft}$. 1bs. of work or energy are required at latitude $45^{\circ}$, sea-level $(g=980.6)$, to raise I lb. of water through $I^{\circ}$ Cent. at $15^{\circ} \mathrm{C}$. For most engineering purposes $1400 \mathrm{ft}$. lbs. would be near enough.

4.190 $10^{i}$ ergs of work or energy are required to raise I gramme of water through $1^{\circ} \mathrm{C}$. at $15^{\circ} \mathrm{C}$.

To reduce these values to any given locality, multiply by the ratio $g_{45}: g$, where $g_{45}$ is the value (980.6) of the acceleration of gravity at latitude $45^{\circ}$, sea-level, and $g$ is the value at the given place. The latter may be obtained from the latitude and altitude of the place by the formula given upon the next page, unless otherwise better known. The altitude correction is but six one-thousandths of one per cent ( 0.00006$)$ for each $1000 \mathrm{ft}$. of elevation, and therefore quite negligible. Within the limits of uncertainty of the quantities involved the latitude correction for places between $30^{\circ}$ and $60^{\circ}$ may be applied thus :-

For esch degree of latitude north of $45^{\circ}$ subtract

For each degree of latitude south of $45^{\circ}$ add

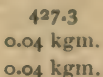

$0.04 \mathrm{kgm}$.
778.8

$0.07 \mathrm{ft}$. Ibs.

$0.07 \mathrm{ft}$. lbs.
1402

$0 . \mathrm{r}_{3} \mathrm{ft} . \mathrm{lbs}$.

$0.13 \mathrm{ft}$. lbs.

Noтr. - The persistence with which the time-honored values, $772 \mathrm{ft} . \mathrm{lbs}$. and $424 \mathrm{kgm}$., of this most important constant are adhered to in practice, although known to be nearly one per cent too small, is due largely to the flagrant negligerice of the authors of text-books of both physics and engineering. No attention is paid to the fact that Joule's original data have been amended acceptully to him, and that his work has been supplemented by the elaborate researches of at least three other independent observers with radically diverse methods. How remarkably these new results check each other and confirm Joule's amended results may be seen from the following table, which is given to indicate the source of the foregoing values.

\begin{tabular}{|c|c|c|c|c|c|c|c|}
\hline \multirow{2}{*}{ A UTHORITY. } & \multicolumn{3}{|c|}{ Original Data. } & \multirow{2}{*}{$\begin{array}{l}\text { REDECED } \\
\text { TO LAT. } \\
45^{\circ} \text { SEA- } \\
\text { LEVEL. }\end{array}$} & \multirow{2}{*}{$\begin{array}{c}\text { DIFFs. } \\
\text { IROM } \\
\text { MEAN. }\end{array}$} & \multirow[b]{2}{*}{ DATw. } & \multirow[b]{2}{*}{ REFTRENOE. } \\
\hline & $\begin{array}{l}\text { J. } \\
\text { kgm. }\end{array}$ & $\theta$. & $t^{\circ} .0$ & & & & \\
\hline \multirow{4}{*}{$\begin{array}{l}\text { Joun (as corrected and } \\
\text { reduced to Baltimore by } \\
\text { Rowland.) [A ssigning eq. } \\
\text { wts, to all methods.] } \\
\text { [A ssigning Rowland's arbi- } \\
\text { trary wts.] } \\
\text { Mean of both. }\end{array}$} & \multirow[b]{2}{*}{$427 \cdot 99$} & \multirow[b]{2}{*}{980.05} & \multirow[b]{2}{*}{$15 .^{\circ}$} & & \multirow[b]{4}{*}{.$- \times 6$} & \multirow[b]{4}{*}{$1847-78$} & \multirow{4}{*}{$\begin{array}{l}\text { See quotations in the } \\
\text { Rowland and Griffiths } \\
\text { references. }\end{array}$} \\
\hline & & & & & & & \\
\hline & 426.66 & 980.05 & $150^{\circ}$ & & & & \\
\hline & $427 \cdot 33$ & 980.05 & $150^{\circ}$ & 427.08 & & & \\
\hline Rowland [at Baltimore]. & $427 \cdot 4$ & 980.05 & $x 5^{\circ}$ & $427 \cdot 16$ & -.08 & 1879 & $\begin{array}{l}\text { Proc. Am. Acad. A. } \\
\text { and } 8, x v, 75(\mathbf{r} 880) \text {. }\end{array}$ \\
\hline $\begin{array}{l}\text { Griffithis [at Greenwich]. } \\
\text { (In ergs.) }\end{array}$ & $4 \cdot 194 \cdot 10^{7}$ & $98 \mathrm{x} \cdot \mathrm{x} 7$ & $150^{\circ}$ & $427 \cdot 70$ & +.46 & 1892 & $\begin{array}{l}\text { Phil. Transac. clxxxiv. } \\
496 \text { (1893). }\end{array}$ \\
\hline Miculescu [at Paris]. & 426.84 & $98 x . \infty 0$ & $150^{\circ}$ & 427.01 & -.23 & $x 892$ & $\begin{array}{l}\text { Ann. de } \mathrm{Ch} \text {, et de } \mathrm{Ph} \text {. } \\
\text { xxvil. } 237(\mathrm{x} 892) \text {. }\end{array}$ \\
\hline \multirow{3}{*}{$\begin{array}{l}\text { Mean of all. } \\
\text { Mesn omitting Joule. }\end{array}$} & & & & $427 \cdot 24$ & \pm .23 & & \\
\hline & & & & 427.29 & & & \\
\hline & & & & $\dot{-}$ & & & \\
\hline
\end{tabular}

The specific heat of water, and therefore the value of $J$, diminishes slightly with rise of temperature. The rate of this diminution is not yet satisfactorily determined, but about as nearly as it is now known the true specific heat $s_{t}$ at any temperature $t^{\circ}$ not far from $15^{\circ} \mathrm{C}$., may be expressed in terms of true specific heat $s_{15}$ at $15^{\circ} \mathrm{C}$. by

$$
s_{\ell}=s_{15}[\mathrm{r} .-0.00030(t-15)] .
$$

Hence $J_{t}$, the number of $\mathrm{kgm}$. or ergs necessary to raise $\mathrm{I} \mathrm{kgr}$. of water from $t^{\circ}$ to $t^{\circ}+1^{\circ}$, will be

$$
J_{t}=J_{15}\left[\mathrm{I} .-0.00030\left(t-\mathrm{I}_{5}\right)\right] \quad\left[\text { Range } 13^{\circ}-20^{\circ}\right],
$$


or for $1 \mathrm{lb}$. of water $1^{\circ}$ Fahr.

$$
J_{8}=J_{60}[1-0.00030(t-59)] \quad\left[\text { Range } 56^{\circ}-68^{\circ}\right] \text {. }
$$

The values of $J_{15}$ and $J_{59}$ are given on pages $7^{1}$ and $7^{2}$. For further tiscussion of this subject consult Griffirus, Phil. Mag. xi. 431 (1895).

The scale of temperature in which these results are expressed is the hydrogen scale of the International Burean of Weights and Measures, which represents, as nearly as it is known, the Thomson absolute scale.

\section{VALUE OF $g$ AT DIFFERENT LATITUDES AND ELEVATIONS.}

$$
g=g_{450}(1-0.00259 \cos 2 \lambda-0.00000020 H) .
$$

$g_{45,0}=980.6 \frac{\mathrm{cm}}{\mathrm{sec}^{2}}$ approx. This is the approximate average value of $g$ at latitude $45^{\circ}$, sea-level. The experimental values vary widely in the next place of figures.

$\lambda=$ latitude of place.

$\boldsymbol{H}=$ altitude of place above sea-level in metres.

Noтz. - Very recent observations render it probable that near the earth's surface the coeflicient of $/ /$ is more nearly 0,00000030 .

\section{BROWN AND SHARPE WIRE GAUGE.}

The diameter corresponding to any gauge number above zero (i.n. of any size less than that of a No. o wire) may be found to within one hundred-thousandth of an inch (five decimal places) by the expression

$$
\begin{aligned}
\left.\begin{array}{r}
\text { Diam. in inches of } \\
\text { gauge number } n
\end{array}\right\} & =0.32486 \times 0.890525^{n,} \\
\text { or, Log. of diam. in inches } & =\overline{1} .51170+\overline{1} .949645 n, \\
\text { or, " " " " } & =9.51170-10 .+(9.949645-10 .) n
\end{aligned}
$$

The diameter corresponding to any gauge numbers $0, \infty$, , larly be computed by the following expressions in which $Y$ is the number of zeros.

Diam. in inches $=0.28930 \times 1.12293^{\mathrm{N}}$,

or, $\quad$ Log of diam. in inches $=\overline{\mathrm{r}} .46134 \mathrm{~S}+0.050 .35 .3 \mathrm{~N}$.

The two primary sizes on which the gauge is based are Nu, 0000, diameter 0.46 inch exactly, and No. 36, dismeter 0.005 inch exactly.

\section{PHYSICAL AND CHEMICAL CONSTANTS.}

For very reliable and extended tables consult L, ismot und B̈̈nswrzis, Physikaliseh Chemische 'Tabellen. 

$\operatorname{sip}^{-140}$ 


$$
51^{1-144^{2}}
$$




\section{PLEASE DO NOT REMOVE \\ CARDS OR SLIPS FROM THIS POCKET}

\section{UNIVERSITY OF TORONTO LIBRARY}

S\&M

A

140 
